\title{
Role of Mahaim fibers in cardiac arrhythmias
}

Citation for published version (APA):

Sternick, E. B. (2004). Role of Mahaim fibers in cardiac arrhythmias. [Doctoral Thesis, Maastricht University]. Datawyse / Universitaire Pers Maastricht. https://doi.org/10.26481/dis.20041215es

Document status and date:

Published: 01/01/2004

DOI:

10.26481/dis.20041215es

Document Version:

Publisher's PDF, also known as Version of record

\section{Please check the document version of this publication:}

- A submitted manuscript is the version of the article upon submission and before peer-review. There can be important differences between the submitted version and the official published version of record.

People interested in the research are advised to contact the author for the final version of the publication, or visit the DOI to the publisher's website.

- The final author version and the galley proof are versions of the publication after peer review.

- The final published version features the final layout of the paper including the volume, issue and page numbers.

Link to publication

\footnotetext{
General rights rights.

- You may freely distribute the URL identifying the publication in the public portal. please follow below link for the End User Agreement:

www.umlib.nl/taverne-license

Take down policy

If you believe that this document breaches copyright please contact us at:

repository@maastrichtuniversity.nl

providing details and we will investigate your claim.
}

Copyright and moral rights for the publications made accessible in the public portal are retained by the authors and/or other copyright owners and it is a condition of accessing publications that users recognise and abide by the legal requirements associated with these

- Users may download and print one copy of any publication from the public portal for the purpose of private study or research.

- You may not further distribute the material or use it for any profit-making activity or commercial gain

If the publication is distributed under the terms of Article $25 \mathrm{fa}$ of the Dutch Copyright Act, indicated by the "Taverne" license above, 


\section{ROLE OF MAHAIM FIBERS IN CARDIAC ARRHYTHMIAS}


(C) Eduardo B. Sternick, Maastricht 2004 ISBN 9052784353

Omslag illustratie: João Prates Romero

Druk: Datawyse / Universitaire Pers Maastricht 


\section{ROLE OF MAHAIM FIBERS IN CARDIAC ARRHYTHMIAS}

\section{PROEFSCHRIFT}

ter verkrijging van de graad van doctor aan de Universiteit Maastricht, op gezag van de Rector Magnificus,

Prof. mr. G.P.M.F. Mols, volgens het besluit van het College van Decanen, in het openbaar te verdedigen op woensdag 15 december 2004 om 12.00 uur

door

\section{Eduardo Back Sternick} geboren te Belo Horizonte, Brazilië

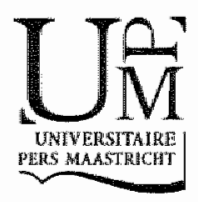


Promotor:

Prof. dr. H.J.J Wellens

Co-promotor:

Dr. Luz-Maria Rodriguez

Beoordelingscommissie:

Prof. dr. H.J.G.M Crijns (voorzitter)

Prof. dr. F.W.H.M. Bär

Prof. dr. R.N.W. Hauer (Universiteit Utrecht)

Dr. J.L.R.M. Smeets

Prof. dr. E.A. Sosa (University of São Paulo, Brazil)

Financial support by the Stichting RESCAR Maastricht and EPTca for publication of this thesis is greatfully acknowledged.

Additional support was granted by: St. Jude Medical do Brasil, Biotronik do Brasil, Medtronic do Brasil, and CardioTek BV, Maastricht, The Netherlands. 


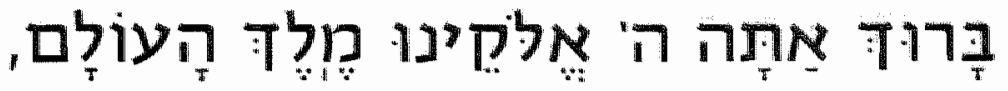

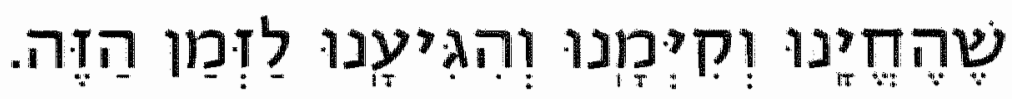

Praised are You, Lord our God, King of the universe, for granting us life, for sustaining us, and for helping us to reach this day.

Para Cláudia, Tomaz, Ivan e Daniel. Aos meus pais Múcio e Nícia. 


\section{CONTENTS}

\section{Chapter 1}

8 Introduction and Classification of the Different Types of "Mahaim" Fibers

\section{Chapter 2}

23 Differential Diagnosis of Left Bundle-Branch Block-Shaped Tachycardias

\section{Chapter 3}

41 The Electrocardiogram in Sinus Rhythm and Tachycardia in Patients with Mahaim Fibers. The Importance of an "rS" Pattern in Lead III

\section{Chapter 4}

56 Tachycardias Associated with Mahaim Fibers

\section{Chapter 5}

75 The Electrocardiogram during Tachycardia in Patients with Anterograde Conduction over a Mahaim Fiber. Old Criteria Revisited

\section{Chapter 6}

88 Fasciculoventricular Pathway: Clinical and Electrophysiological Characteristics of a Variant of Preexcitation

\section{Chapter 7}

99 The Electrocardiogram in Patients with Fasciculoventricular Pathways. a Comparative Study with Anteroseptal and Midseptal Accessory Pathways

\section{Chapter 8}

112 Mahaim fiber: an Atriofascicular or a Long Atrioventricular Pathway? 


\section{Chapter 9}

121 Dual Conduction in a Mahaim Fiber

Chapter 10

130 Appraisal of Mahaim Automatic Tachycardia

Chapter 11

141 Automaticity in Mahaim Fibers

Chapter 12

154 Short A-V Mahaim Fibers

Chapter 13

170 Summary and Conclusions

174 Sumário e Conclusões

179 Samenvatting en Conclusies

183 Acknowledgements

185 Curriculum Vitae

186 Publications 


\section{Chapter 1}

\section{Introduction}

Contents

1.1 Historical Remarks on Variants of Preexcitation

1.2 Diagnostic Aspects

1.3 Therapy

1.4 References 


\title{
HISTORICAL ASPECTS OF THE VARIANTS OF PRERCITATION
}

Almost twenty five years ago, Gallagher et all stated that "whe vole of Mahaim fibers in the genesis of cardiac arthymas in man has been comtroversial since the were first described ". The original description was made in the late 30 's by Mahaim" $^{23}$ (Figures 1 and 2).

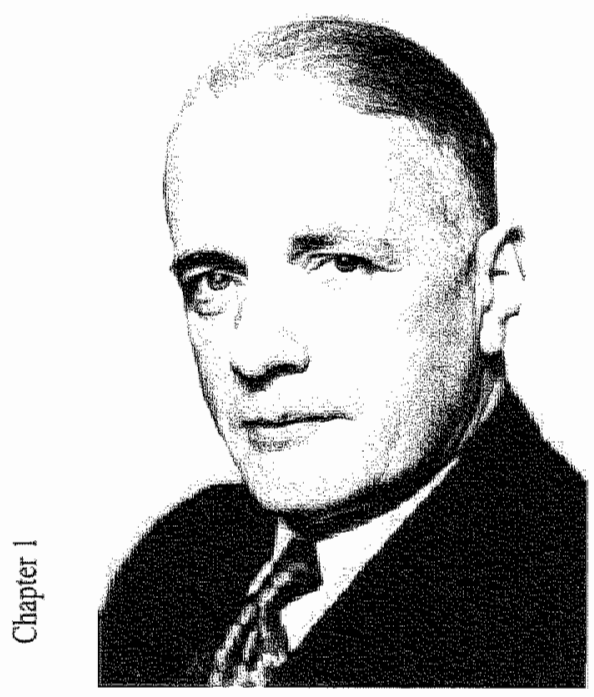

\begin{abstract}
Figure 1: Wam Mahan (1897-1965). Born

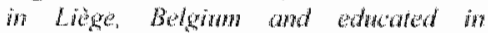

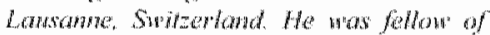

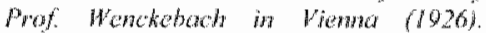

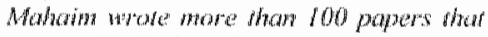

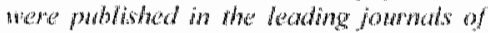

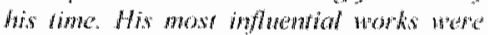

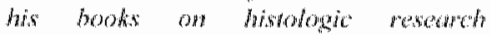

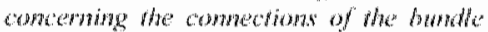

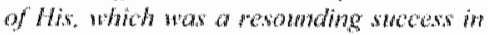
Exrope in 1937 an if promed the has for

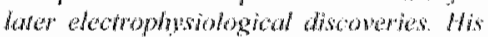

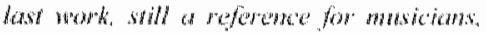

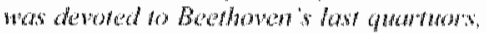
and porblished in 1964.
\end{abstract}

He found conducting tissue extending from the A-V node to the ventricular myocardium. There are only a few articles dealing with anatomicall studies ${ }^{3-8}$ of Mahaim fibers. Early investigators found that Mahaim fibers were accessory connections taking off from the His bundle and fascicles ( $F$-fasciculoventricular) into the right ventricle or from the atrioventricular node (NV-nodoventricular libers) to the right ventricle or to the right bundle (NF-nodofascicular fibers). Anderson ot al proposed 2 varieties of NV fibers, one arising in the transitional zone and the other taking off from the deep, compact nodal portion of the $\mathrm{AV}$ junction. The nodoventricular concept was consistent with the findings from the Gallaghen series where some patients had $\mathrm{V}$-A block during wide complex tachycardia, proving that in some patients the atrium was not pant of the reentrant circuit.

Wellens $^{10}$ was the first to report on the electrophysiologic findings using the technique of programmed electrical stimulation in a patient with an accessory pathway with decremental properties and long conduction times, alssuming a relationship with the fibers described long ago by Mahaim. The term nodofascicular (NF) was applied when the retrograde His bundle potential preceded the ventricular deflection, while a nodoventricular pathway was assumed when the retrograde His bundle deflection followed the beginning of the ventricular potential. The nex step 


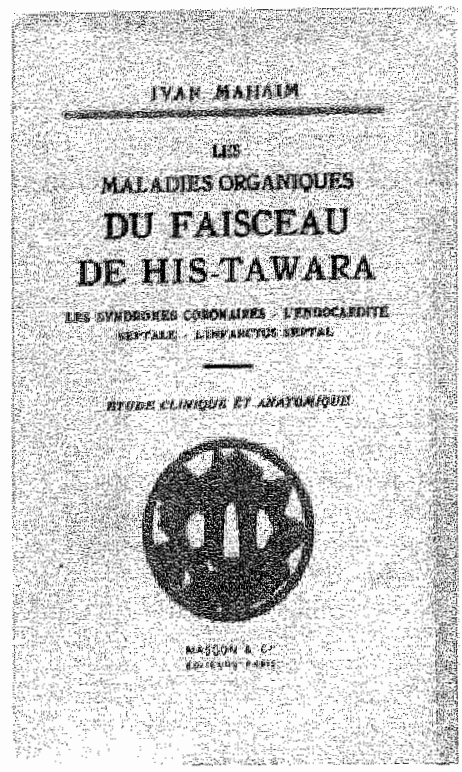

Figure 2: Les Maladies Organiques du Fascenalle de His-Tawara: This book is shill used as a neforence tolat and nos dewoted to the thrnors of we heart. providing a dewted anatysis of wore Ihan 400 cases. II was prblished in 1931 and anricipares the possinithy of cardiac

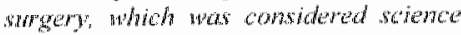
frotion by thers.

was the understanding of the functional significance and the anatomicelectrophysiologic relationship of such pathways. An important observation was made in 1978 by Becker et $\mathrm{al}^{7}$ when they described an accessory A-V node associatedwith a bundle of specialized fibers measuring $1 \mathrm{~cm}$, coursing through the right ventricle, mimicking a second $A-V$ conduction system located on the lateral tricuspid anmulus. However, that finding did not change the mainstream concept of NV libers at that time. During the early 80 's many centers started to refer patients with drug refiactory tachycardias due to Mahaim fibers for surgical treatment. Although Gillette et al" reported as early as 1982 a Mahaim fiber located on the anterior portion of the tricuspid ring, according to the current concepts at that point in time, ablation of the A-V node was considered to be the logic strategy for curative treatment of patients with NV/NF fibers. Some electrophysiologists introduced a new technique delivering high-energy current through a catheter to achieve ablation of the A-V node to treat a patient with a Maluam fiber. This resulted in complete A-V block but persistent preexcitation ${ }^{2}$. The turning point came in 1988, when Klein, Guiraudon and colleagues ${ }^{13}$ decided to extensively freeze the $\mathrm{A}-\mathrm{V}$ node and upper His bundle region of a 29 year old man and discovered that preexcitation did not go away. That finding indicated to them that the accessory pathway was not connected with the $A-V$ node. In the next patient the $A-V$ node was not damaged but the Mahaim tiber successfully blocked by ice mapping at the right lateral aspect of the tricuspid annulus. Klein's manuscript was published in 1988. Shortly thereafter Tchou et al" published a paper entitled "Arrofascicular comection or a nodoventricular fiber? Electrophysiologic elucidation of the pathway and assoctated reentran circuir." In this elegant study the authors describe a simple maneuver to 
prove that such pathways are in fact inserting in the atrium and not in the A-V node by delivering a late lateral atrial premature beat at the the that the $A-V$ node was refractory. They showed that it was possible to advance verntricular depolarization during preexcited tachycardia. In the following years, catheter ablation techniques shed more light on the subject. Discrete high frequency potentials resembling the His bundle potential, considered to be the ellectrical activation of Malham tissue, were used as an effective target for ablation ${ }^{15}{ }^{16}$. Observations during pharmacological interventions " histologic data. ${ }^{7}$. $18-1 \%$ "electrophysiologic maneuvers and findings during radiofrequency catheter ablation like heat induced automaticity ${ }^{22} .23$, and spontaneous automaticity ${ }^{24}$ were presented as further evidence that the Mahaim fiber is composed of A-V nodal like tissue. Some authors ${ }^{20}$ believed that an accessory $A-V$ node without a direct connection to the ventricle, can be the substrate of atrial tachycardias mapped to the lateral tricuspid ring. They showed potentials preceding the P wave and decremental conduction between those potentials ("M") and the P wave and also the occurrence of automaticity during radiofrequency catheter ablation. The NV/NF fibers are now considered a rare item. Anecdotal reports suggest ${ }^{25}$ their presence in some patients with narrow and regular QRS tachycardia with A-V dissociation.

Recent reports using non-contact technologies for intracardiac mapping of Mahaim fibers like the EnSite ${ }^{26}$ and the LocaLisa system ${ }^{27}$ have validated old data derived from open heart epicardial mapping and intracardiac catheter mapping ${ }^{1 .}$ 1. $^{\text {, }}$ suggesting that Mahaim fibers are long structures connecting the right atrium to the anterior apical region of the right ventricle, close to or inserting into the distal part of the right bundle branch.

The variety which is known as fasciculoventricular pathways ${ }^{2 *}$ are not related anatomically to atriofascicular pathways. They do not have long conduction times nor decremental properties. As long as they are infra $A-V$ nodal structures connected to the His butndle or its fascicles the decremental conduction is coming from the AV node which led previous investigators to believe otherwise. They play no role in clinical tachycardias. However, due to the fact that its pre-excitation pattem on the 12-lead electrocardiogram may resemble that of an anteroseptal accessory pathway. and they are often associated with rapidly conducting bypass tracts, a misdiagnosis of a bypass tract should be avoided to prevent unnecessary damage to the $\mathrm{A}-\mathrm{V}$ node-His bundle conduction system by catheter ablation.

\section{CRITICAL ANALYSIS OF THE CLASSIFICATION OF MAHAIM FIBERS}

The preexcitation syndromes were originally classificd on the bess of their anatomic location and course and named according to the original investigators resulting in Mahaim, James, and Ken fibers. Later, such a description was considered inadequate because it did not fit the new electrophysiologic or anatomic knowledge. The European Study Group for Preexcitation introduced in 1975 a new classification based upon the proposed anatomic connections of the accessory pathways. However, the eponym Maham fiber survived the European Study Group proposed changes. Why? It seems that the major issue here is the common electrophysiologic finding of long and decremental conduction properties (A-V node like behavior) of such pathways, the so called "Mahaim" physiology. 
An updated anatomical classification of the Maham fibers is shown in figure 3 :

Proximal insertion in the AV node - His - Bundle branch system

1. Nodofascicular bypass tract

2. Nodoventricular bypass tract

3. Fasciculoventricular pathway

Proximal insertion in the atrium

1. Atriofascicular pathway

2. Short atrioventricular pathway with prolonged and decremental conduction

3. Long atrioventricular pathway with prolonged and decremental conduction

4. Left-sided atrioventricular or atriofascicular pathway with prolonged and decremental conduction

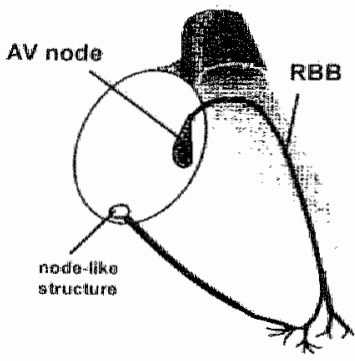

atriofascicular

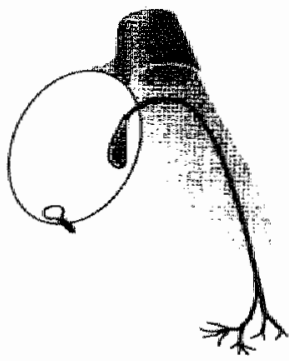

short atrioventricular long atrioventricular

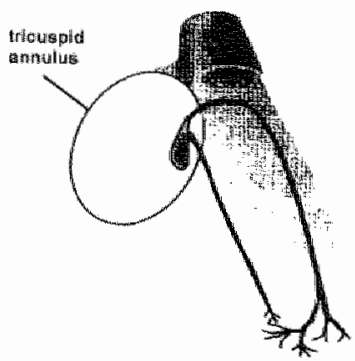

nodofascicular

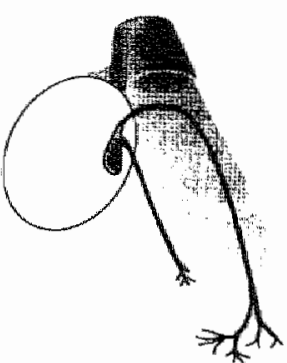

nodoventricular

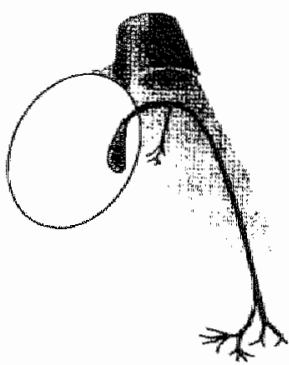

fasiciculoventricular

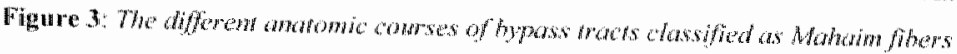




\section{DIAGNOSIS}

ELECTROCARDIOGRAPHIC FEATURES: The baseline electrocardiogram of patients with Mahaim fibers (figure 4) is characterized by minimal (ORS width less than $0,12 \mathrm{sec}$ ) or no ventricular pre-excitation.

A

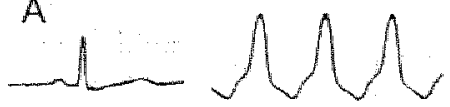

Non
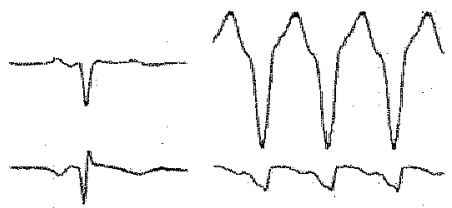

है
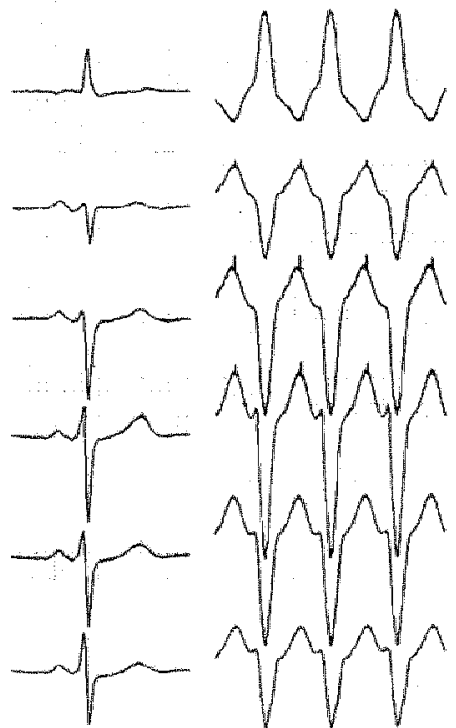

u1

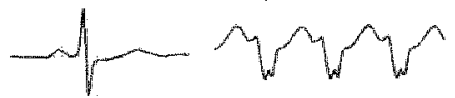

- Mromprom

II

U3

45
B
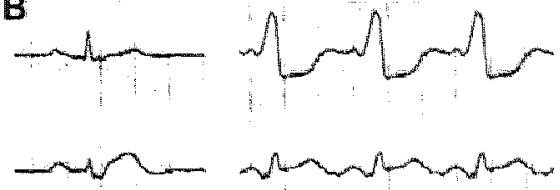

IIII

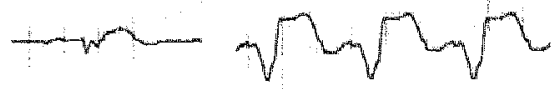

คum

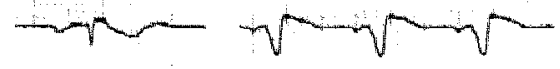

AUL

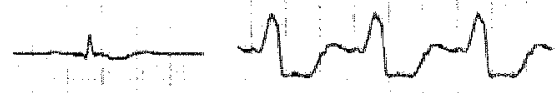

ติน
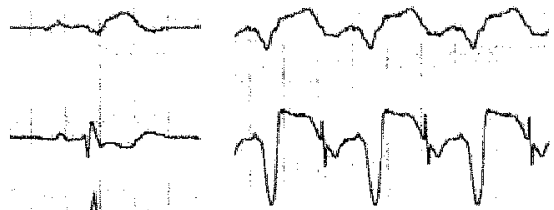

U2
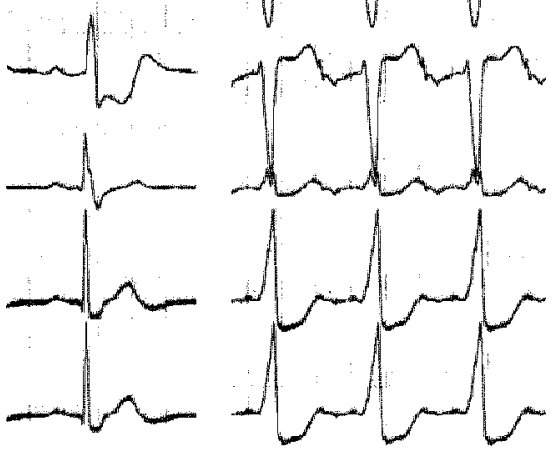

16
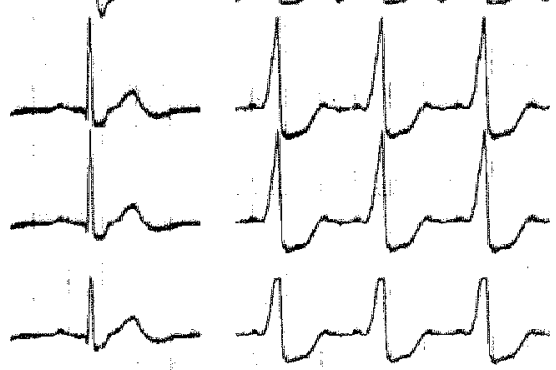

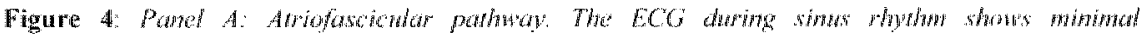

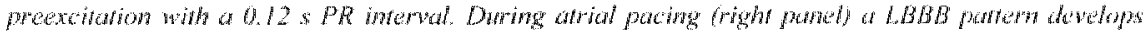

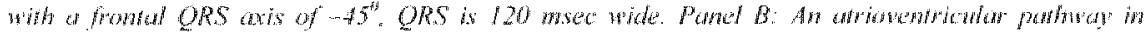

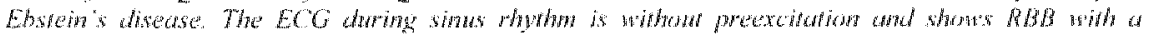

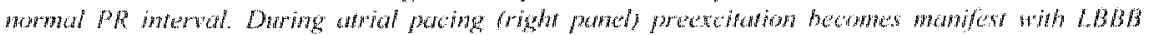

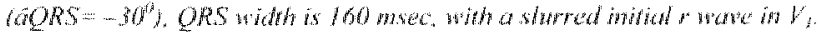


Sometimes the only clue is the absence of a septal $q$ wave in leads 1 , $V_{5}$ or $V_{6}^{29}$. An $\mathrm{rS}$ pattem (less frequently rs $\mathbb{R}^{\text {) }}$ in lead III was found in 28 of 40 patients (70\%) with a Mahaim fiber (figure 5 ).
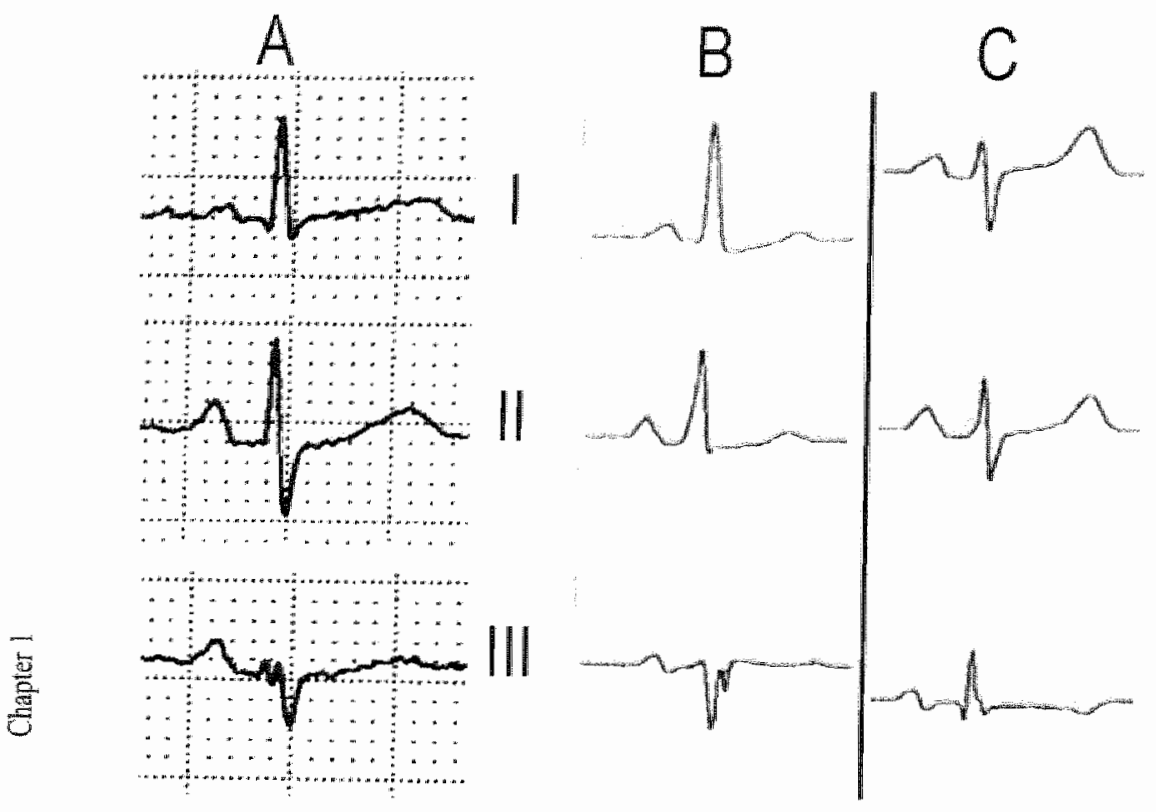

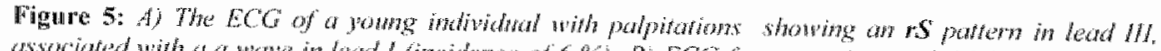

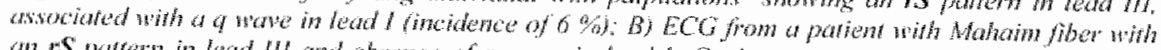

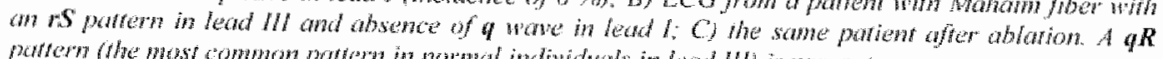

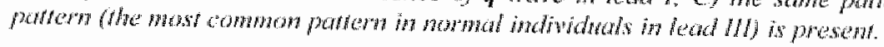

Because a nomal person may also have such an $\mathbf{r S}$ pattern in lead 111 , we did a survey in 200 young individuals (mean age of 23 years) to address the issue of the specificity of this QRS pattern in lead III. It was found in $6 \%$ of the 200 . However, no individual had an rS pattern in lead III with absence of a $q$ wave in lead $\mathbb{I}$. We conclude that the association between the absence of a $q$ wave in lead I and an is pattern in lead III seems very specific lor Mahaim. Patients with a Mahaim fiber having an abnormal pattern in lead III show left axis deviation in the frontal plane during antidromic tachycardias. After successful catheter ablation a different ORS pattern usually emerges (most commonly a $q \mathbf{R}$ or $Q R$ pattern in lead III). It is worth mentioning that the rS pattern can be concealed by an associated WPW and emerge after the successful ablation of the rapidly conducting accessory A-V pathway, as did happen in 4 out of 5 patients in our cohort. Therefore in patients with paroxysmal tachycardias the presence of an $\mathrm{r}$ pattern in lead IIll during sinus rhythm should raise 
the suspicion of a Mahaim fiber. Occasionally, patients, particularly those having a short atrioventricular accessory pathway with a long conduction dime show a typical $\angle B B B$ pattern with a normal PR interval. Some patients are unable to have anterograde conduction over the Mahaim fiber at rest and even during atrial pacing". In spite of that, these so called "Iatent" Mahaim fibers may become engaged in antidromic tachycardia. The differential diagnosis from ventricular tachycardia can then only be achieved by performing an electrophysiologio study.

Variability in minimal preexcitation may occur either on the same ECG or on ECG's taken on different days. This variable ECG presentation does not correlate with a long refractory period, in contrast to intermittent preexcitation occurring in patients with rapidly conducting accessory pathways.

The ECG during a tachycardia with anterograde conduction over an atriofascicular fiber shows a typical LBBB patten with a frontal axis usually ranging from $-0^{\circ}$ to $60^{\circ}$. While the axis is of no help in differentiating it from $\mathrm{A}-\mathrm{V}$ conduction over a right sided $A-V$ pathway, the QRS complex is rather narrow $(0,12-0,14$ seconds) being larger when anterograde $A-V$ conduction proceeds over a short atrioventricular Mahaim. In the latter situation there is often a broader $r$ wave in $V_{1}$ and $V_{2}$ $(>30 \mathrm{msec})^{3}$. Bardy's ${ }^{32}$ eriteria for an atriofascicular Malhaim remain useful today: $R$ wave in lead $I$, $r S$ in $V_{1}$, QRS width $\leq 0,15$ seconds, precordial $R / S$ transition after $V_{4,}$ a QRS frontal plane axis between 0 and $-75^{\circ}$ and a tachycardia cycle length between 220 and 450 msec. We have validated the abovementioned criteria ${ }^{33}$ (except cycle length) only for atriofascicular pathways, but not for short A-V Mahaim's. The electrocardiogram of a fasciculoventricular pathway is characterized by a normal QRS frontal plane axis as in an anteroseptal accessory AV pathway $\left(0 \text { to }+75^{6}\right)^{3 \mathrm{ki}}$ with subtle preexcitation and a normal PR interval. A short PR interval may be present in case of enhanced A-V nodal conduction (figure 6).

Atrial pacing does not change the degree of preexcitation. Junctional beats are preexcited and intravenous adenosine results in blocked $\mathrm{P}$ waves. The R/S>1 precordial transition usually occurs in lead $V_{2}$.

ELECTROPHYSIOLOGIC CHARACTERISTICS: The major finding is the presence of a pathway with slow conduction and decremental properties cluting right atrial pacing ancreasing rates or after atrial premature beats. The $\mathrm{AH}$ interval lengthens, the HV interval shortens and the QRS widens until a steady value is reached. A further increase in atrial stimulation ate does not imcrease preexcitation, but can prolong $A-V$ conduction until A-V block occurs.

Decremental conduction is usually defined as rate dependent prolongation of the conduction time over an $A-V$ accessory pathway of nore than $30 \mathrm{msec}$, as measured using electrograms close to the $\mathrm{AP}$ insertion. During atrial pacing at increasing rates achievement of maximal preexcitation is usually associated with retrograde conduction over the right bundle, His bundle and A-V node and then followed by antidromic lachycardias when pacing is stopped. An important step is to assess the role of the accessory pathway in the tachycardia circuit: active or bystander. This can be done by giwing single late laterally located right atrial 

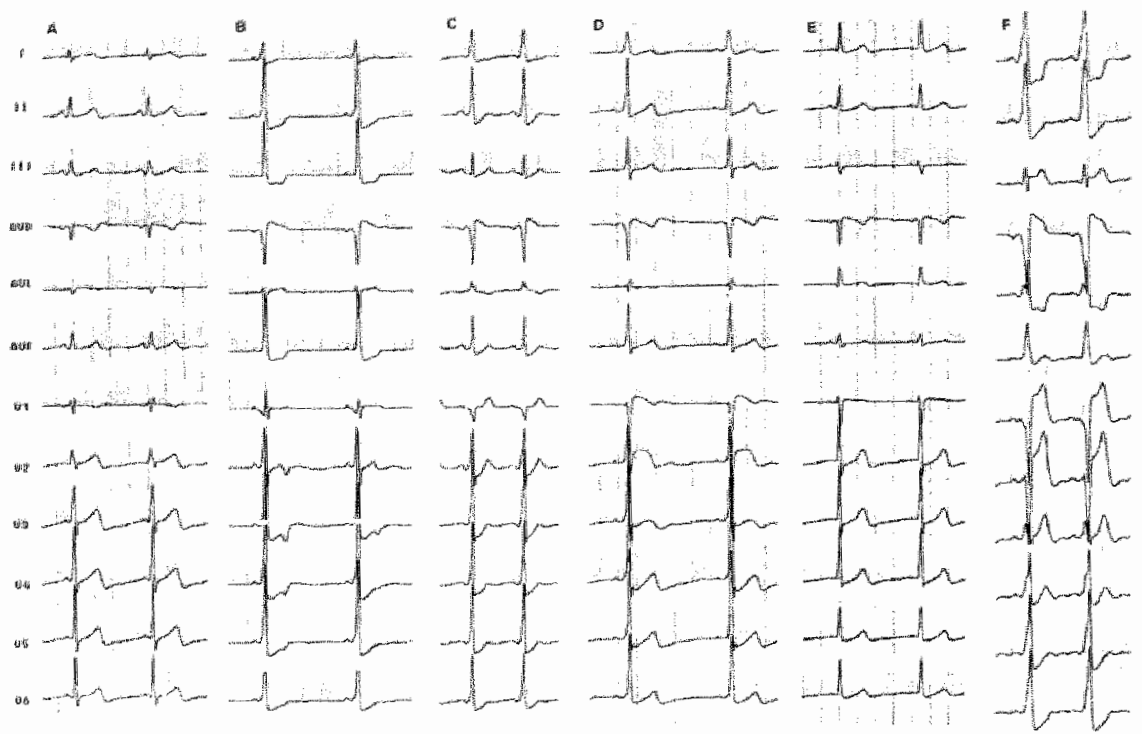

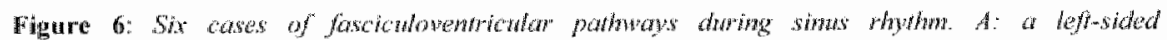

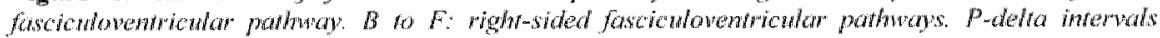

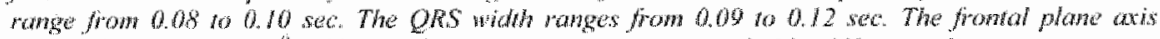

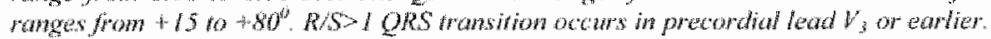

extrastimuli during a preexcited tachycardia, not affecting the His bundle and the atrial electrogam at the coronary sinus ostrum (figure 7). Advancement of the QRS complex and subsequent atrial activation establishes the diagnosis of an extranodal accessory $A-V$ pathway (having a proximal atrial insertion) as well as incorporation in the tachycardia circuit.

If advancenent of QRS activation occurs without changing the timing of atrial activation, the presence of an extranodal $\mathrm{AP}$ is certain but its participation in the circuit still necd to be proved.

MeClelland et al ${ }^{\text {th }}$ could successfully advance QRS activation with late right atrial extrastimuli in 22 of 23 patients with an atriofascicular tachycardia. Another less elegant maneuver proving AP participation in SVT is by having catheter-induced RBBB. Assuming an antidromic tachycardia incorporating an atriofascicular for atrioventricular) pathway inserting nto the right ventricle retrograde conduction during the tachycardias occurs via the right bundle-His bundle and $A-V$ node axis. $A$ RBBB will lengthen the circuit thereby increasing the tachycardia cycle length because of ventriculo-atrial conduction over the left bundle branch. 
Preexcited $A-V$ nodal reentry as well as antidromic tachycardia with retrograde conduction through another AP will not be affected by RBBB. The proximal atrial insertion can also be localized with the recording of an accessory pathway potential, usually recorded in the lateral aspect of tricuspid anmulus, away from the $A-V$ node (figure 8). In the study by Grogin et a ${ }^{3.5}$ clues as to the presence of a nodoventricular fiber were the inability of a premature atrial stimulus to advance the ventricle and the presence of dual $A-V$ nodal pathways.

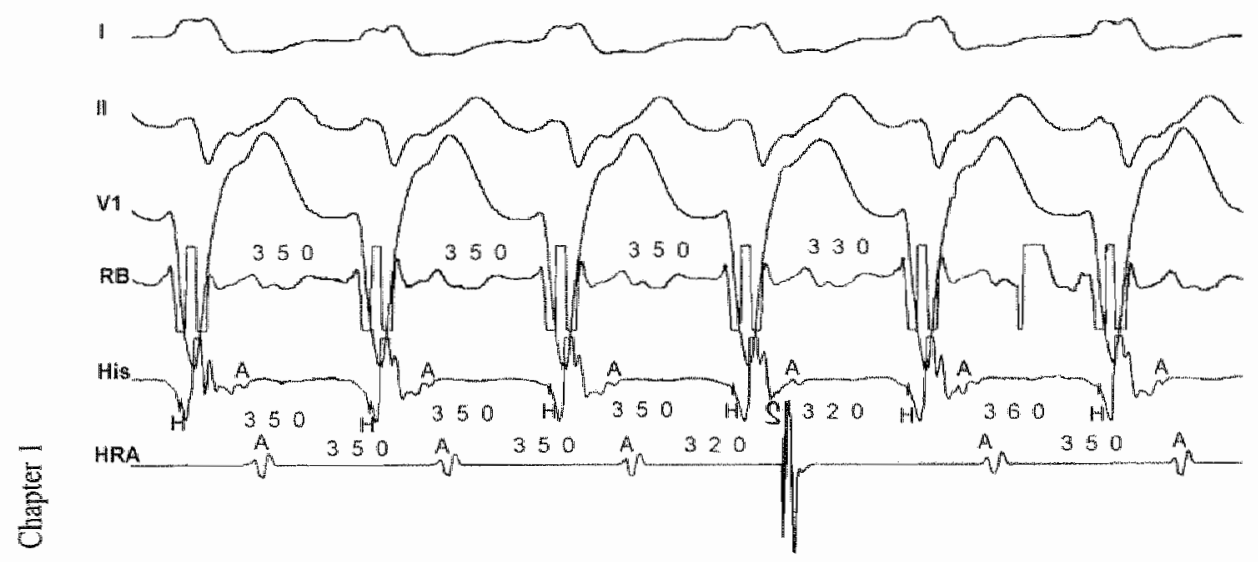

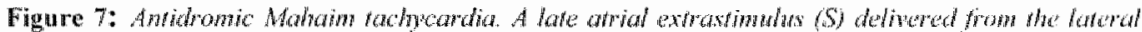

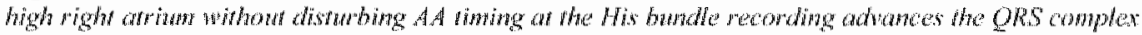

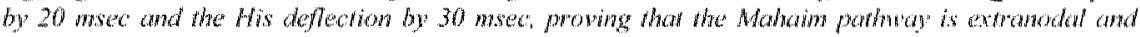

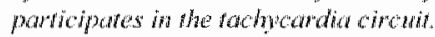

Scheinman et $a^{36}$ described a patient with a lateral Mahaim in whom an atrial premature beat could not preexcite the ventricle. However, the finding of an " $M$ " potential and successful ablation at this spot was consistent with an atriovantricular Mahaim fiber and not a nodoventricular fiber. Ventricular stimulation ustally discloses ventriculo-atrial conduction over the A-V node. The vast majority of atriofascicular and atrioventricular Mahaim pathways are unable to conduct retrogradely ${ }^{37.38}$.

Adenosine injection during sinus thythm usually causes block of impulse conduction over both the A-V nodle and the Mahaim fiber (fugure 9). Occasionally it can show a more marked effect over the $A-V$ mode and increase preexcitation ${ }^{3 \%}$. Verapamil ${ }^{17}$ has a more marked effect on $\mathrm{A}-\mathrm{V}$ nodal conduction, and is more helpful in exposing preexcitation. During antidromic tachycardia adenosine causes termination of antidromic tachycardia by prolongation of conduction over the Mahaim pathway and block. 


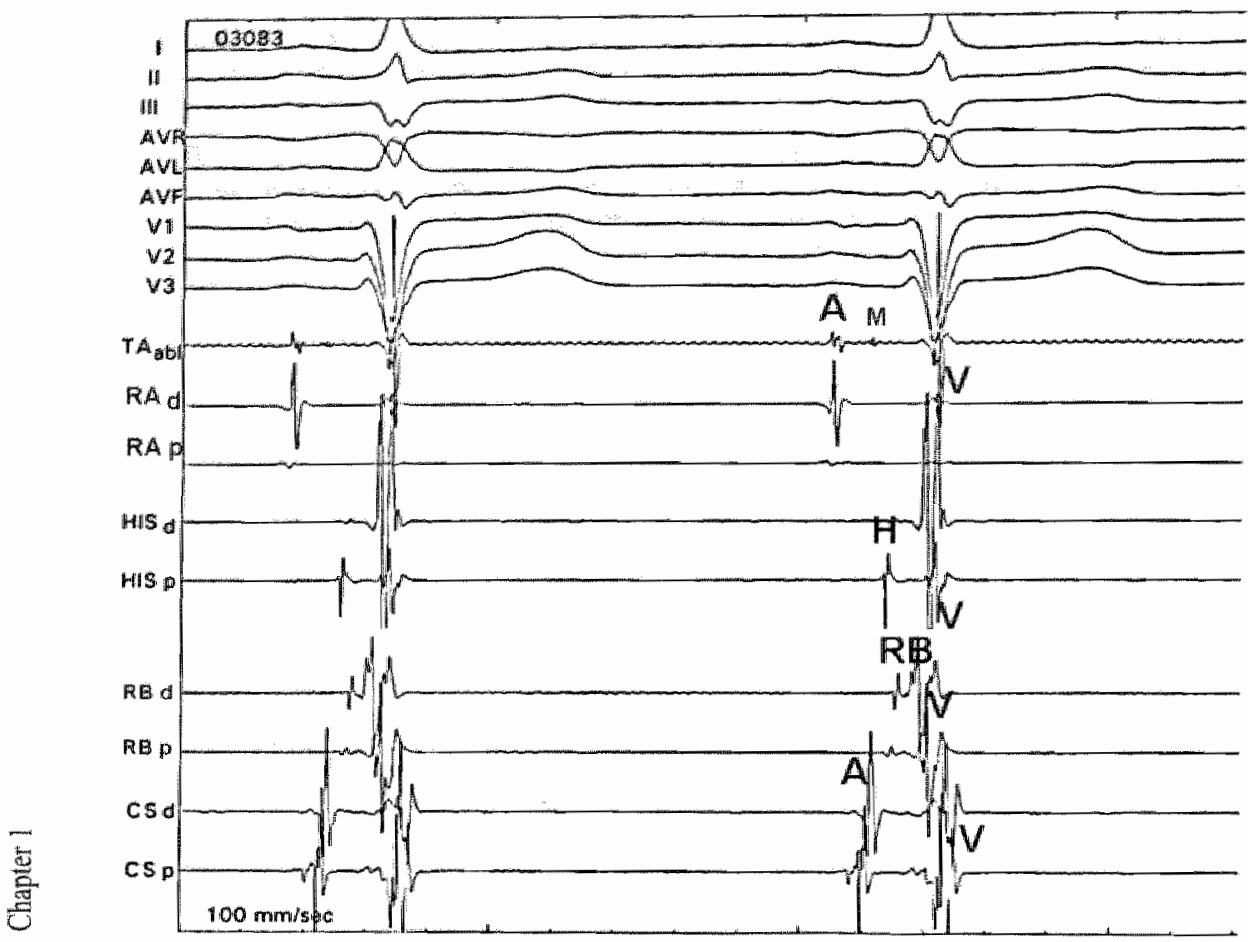

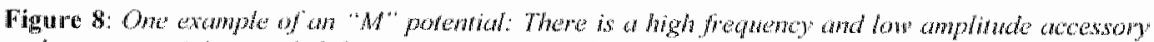

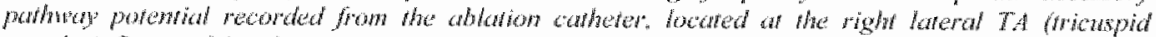

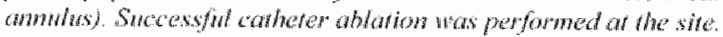

Mahaim fibers often occur in the setting of Ebstein"s disease $(20 \%)$ and associated rapidly conducting accessory pathways (usually right sided) are seen in up to $30 \%$ of these patients. Fasciculoventricular pathways also occur wery often in association with rapidly conducting bypass tracts. In cases reported since $1981^{1,26,40,41}$ there was a $4.0 \%$ incidence of an assoeiated $\mathrm{AP}$.

\section{TREATMENT}

ANTIARRHYTHMIC DRUG TREATMENT: In general, verapamil and propranolof have been ineffective for termination of tachycardia episodes ${ }^{17}$. Intravenous adenosine is the first choice intravenous drug for acute treatment during Mahaim tachycardia. Patients with Mahaim fibers are frequently unresponsive to multiple antiarrhythmic drugs. Flecainide, propalenone, propranolol, sotalol, and digoxine have been used as single drugs or in combination with little success. Some authors reported that type $\mathbb{A}$ A agents (quimidine ${ }^{+2}$ ) were effective in preventing or slowing 
tachycardia. Cumently, antiarrhythmic dng therapy plays a secondary role becouse of the highly successfull results with radiofrequency catheter ablation.

MAPPING AND CATHETER ABLATION: Sonte features are unique to Mathaim fibers: Mapping of the atrial insertion during ventricular stimulation is usually not possible because these decrementally conducting pathways ustally do not conduct retrogradely. Excentric ventriculo-atrial conduction if present, may indicate associated rapidly conducting bypass tracts. A short atrioventricular Mahaim can be located by mapping the site of earliest ventricular activation at the annulus, as with other anterogradely conducting accessory $\mathrm{A}-\mathrm{V}$ pathways. In atriofascicular pathways or long atrioventricular Mahaim"s the distal (non-annular) insertion camot be mapped in this way. To worsen matters these decremental pathways are unusually sensitive to mechanical trauma. Accidentally touching the annulus with the ablation catheter can result in transient abolition of conduction through the pathway lasting minutes to hours ${ }^{43-4 t}$. The following strategies have been used to overcome these problems:

1. Searching for the "M" (Mahaim) potential (figure 8) along the tricuspid annulus is the most commonly used technique. The ablation catheter should be moved

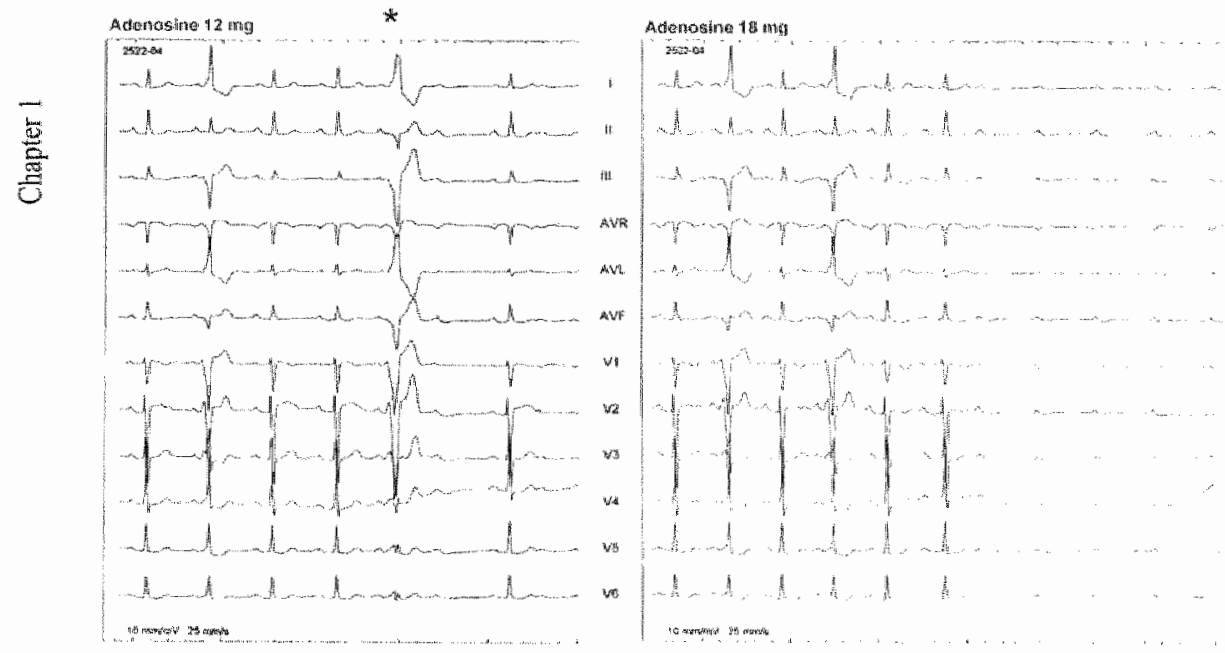

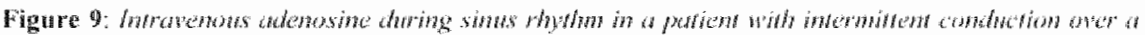

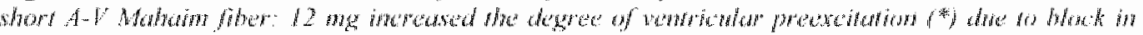

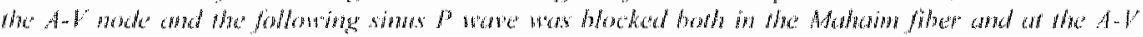

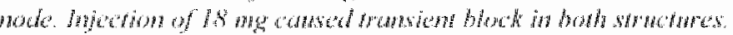


cautiously along the anmulus awoiding bumps on the Mahaim tissue. The potential may be as large as the His bundle potential or small and nartow with low amplitude. Catheter ablation at a site with an "M" potential is likely to be successful. We ${ }^{23-24}$ and others ${ }^{22} \cdot 37$ have recorded automatic rhythms (Mahaim automatic tachycardiasMAT) during radiofrequency current delivery in most patients with Mahaim fibers ( 30 out of 33 patients: $91 \%$ ). It is probably due to heat-related automaticity of nodallike tissue ats is also seen during slow $A-W$ nodal pathway ablation. MAT is in most cases short-liwed but may sometimes last up to 2 minutes. At least in some patients it is necessary to completely terminate those rhythms to prevent recurrences.

\section{Aleferences}

1. Gallagher JJ, Smith WM, Kassell JH, et al: Role of Mahaim fibers in cardiac arhythmias in man. Circulation $1081 ; 64: 176-189$.

2. Luderita B. Waan Mahaim: J lun Card Electrophysiol 2003:8:155.

3. Mahaim 1. Benait A: Nouwelles recherches sur les connexions stupérieures de la branche gauche du Giscean de His-Tawara avec cloison interventriculaire. Cardiologia 1938:1:61-76.

4. Maham I, Winston MR: Recherches danatomie compare et de pathologie experimentale sur le connexions hantes du labiscean de His-Tanrara. Cardiologia 1941;5;189-260.

5. Lew M, Gibson S, Miller RA: Ebsten's disease with Wolff-Parkinson-White syndrome. Am Heart $1195,79,724-741$.

6. Lew M, Sodi-Palhatres D, Priedlan C: A histopathalogic study of the atrioventricular communications in a caste of WPW with incomplele left bundle branch block. Am Heart 1 $1963 ; 66: 399-404$.

7. Becker AF, Anderson RH, Durrer D, Wellens HJ: The anatomical substrates of WollfParkinson-White syndrome: a clinico-pathologic correlation in seven patients. Circulation $1978 ; 57: 870-879$.

8. Anderson RH, Becker AE: Stantey Kent and accessory atrioventricular connections. I Thoracie Cardiovatse Surg 1981;81:649-658.

9. Anderson RH, Becker AE, Brechenmacher $\mathrm{C}$, et al: Ventricular preexcitation. A proposed nomenclature for its substrates. Eur J Cardiol 1975,3:27.

10. Wellens HJ. Floctrical Stimulation of the hear in the study and ireatment of tachycardias. Uniwersily Park Press, Ballimore, 1971: p 97-10\%

11. Gilietle PC. Garson A, Coolcy DA, at al: Prolonged and decremental anterograde conduction properties in right anterior accessory connections: wide QRS ant idromic tachycardia of left bundle bancla block puttern without Woll-parkinson-White condiguration in sinus thythm. Am Heart I
$19821103,66-74$.

12. Bhandari A, Mordy I. Shen EN, at al: Catheter-indueed this burdle ablation in a patient with reentrant tachycardia associated with at nodowentricular tract. I Anr Coll Cardiol 1984;4611-616

13. Klein GJ, Guiraudon GM. Kerr CR, el al: "Nodoventricular" aceessory pathway: evidence for a distind acessory atroventricular pathwaly with atroventricular node-like propertes. 1 Am Coll
Cardiol $1988: 11: 1035-1040$.

14. Tchou P. Lelmann MH, dazayeri M. Akhtar M: Atriofiscicular comection or a nodoventricutar

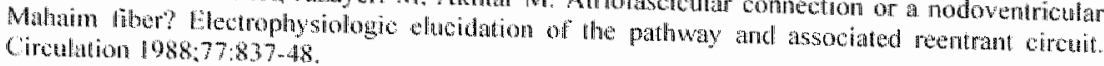

15. Brugada $I_{x}$ Mantinez-Sanches y, Kuzmicic B. et atl: Radiofrequency catheter ablation of

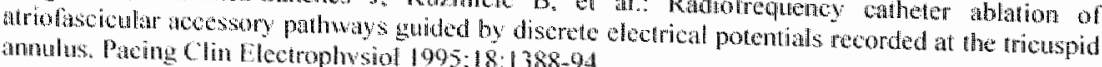


16. Mcclelland $J H_{x}$ Wang $X_{*}$ Beckinan $K J$, at: Radiofrequency atheter ablation of right atriofascicular (Mahaim) accessory pathways guided by acessory pathway activalion potentials. Circulation $1994,89: 2655-56$.

17. Ellenbogen KA, Rogers R, Old W: Pharmacological chancterization of conduction awer a Mahaim fiber; evidence for adenosine sensitive conduction. Puchn Clin Electrophysiol 1989:12:1396:1404.

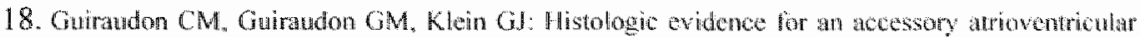

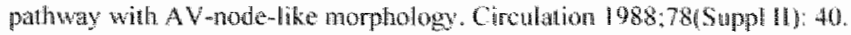

19. Epstein MR, Satul JP, Weindling SN, et all: Atrioventricular reciprosalng taclaycardia imolving twin atrioventricular nodes in pattents with complex congental heart disease. I Cardiowase Electrophysiol 2001:12:671-679.

20. Nogami A. Suguta M. Tomila T, et al: Nowel form of atrial tachyoardia originathing at the atroventricular annulus. Pacing Clin Electrophysiol 1998:21:2691-94.

21. Gollob MB, Bharat S, Swerdlow CD: Accessory atriowemtricular node with properties of a typical accessory pathway: Anafomic-eloctrophysiologie correlation. I Cardiowac Electroplysiof $2000: 11: 922-6$

22. Braun E, Siebbels J, Volkmer $M$, ef al: Radiofrequency-induced preexcited autonatic thythm during ablation of accessory pathways with Mahaimmpe preexcitation: does it prediets climical outcome? Pacing Clin Electrophysiol 1997:20(4) Part 11:1121

23. Sternick EB, Gerken LM, Vrandecic MO. Appratsal of "Mahaim" automatic tachycardia I Cardiovase Electrophysiof 2002:13:244-249.

24. Sternick EB, Tümnernans $C$. Sosa E, et al: Automaticity in Mahaim fobers \& Cardiowaso Electrophysiol 2004:15:738-744

25. Huchy J, Schickel S, Jörger U, et al: Electroplysiologic chatacteristics and badiofrequancy ablation of concealed nodofiseicular and left anterograde atriofascicular pathways. I Cardiovase Electrophysiol 2000;11:211-217.

26. Fung WHJ, Chan HCK, Chan WWL, Sanderson IE. Ablation of the Mathaim Pathway guided by noncontact mapping. I Cardiovase Electrophysiol 2002;13:1064.

27. Tan HL. Wittkanpf EHM, Nakagawa H, Derksen R. Arrofascicular accessory pathway, I Cardiovase Elecirophysiol 2004:15:118.

28. Sternick EB, Gerken LM, Vrandecic M, Wellens HJ.J. Fasciculowentricular Pallwways: Clinical and electrophysiologic characteristics of a variant of preexcitation. I Cardiovase liectrophysiol $2003 ; 14: 1057-63$.

29. Haissaguerre $M$, Cauchemez $B$, Marcus $F$, et al. Characteristics of the ventricular insertion siles of accessory pathways with anterograde decomental condwetion properies. Circulation $1905,91: 1077-1085$

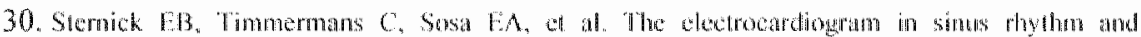

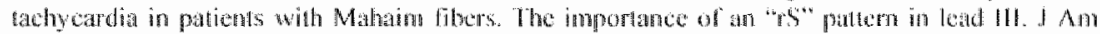
Coll Cardiol 2004:44:1626-1635

31. Davidson NC. Morton JB, Sanders P, Kalman J: Lanen Mahaim fiber als a cause of antidesenic

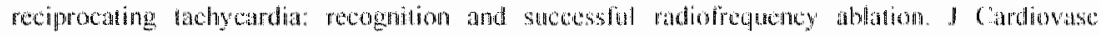
Electrophysiol 2002; 3:74-8.

32. Bardy GH. Fedor JM, German LD, en al: Surfice chectrocardingraphic clueos suggesting presence of a modofaseicular Mahaima fiber. 1 Am Coll Cardiol 1984,3:161-1168.

33. Sternick 1B. Craz FES, Timmermans C, Sosa I:, Rodrigue\% I.M, Gerken I.M, Fagundes MI. Scanavarea M! Welens IVI. Hear Rhy lhm 2004;/44):406-413.

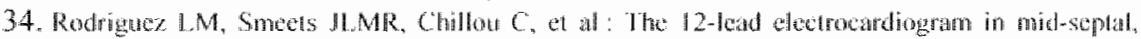

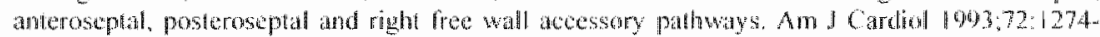
1280.

35. Grogin HR, Lee RJ, Kwasman M, el al. Radiofrequency enthefer abiation of alrobliseicular and nodosentricular Mahaim tracts. Greulation 1994:90:272-281. 


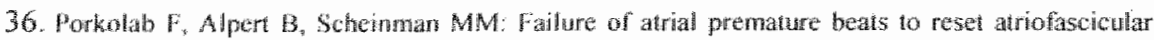
tachycardia. PACE 1999;22:528-530.

37. Peinado R, Merino It. Ramire L. Echeverta I: Decremental aldiofascicular accessory pathway whih bidirectional conduction: defineation of atrial and ventricular insertion by radiofrequency current application. J Cardiovascular Elecirophysiol 2001;12:489-492.

38. Anaderio $M_{3}$ l. uzzi $G$. Forleo $C$, ef al: Radiofreduency catheter ablation of a biditeetional decremental acossory atrioventricular pathway in the coronary sinus. Cardrologia 1999;44:89-95.

39. Bethassen $B$. Glick A. ORS complex with a lefi bundle branch block morphology during adenosine triphosphate tewt: what is the diagnosis? J Cardiowase Electrophysiol 2002;13519-520.

40. Ganx LI, Elson J. Chenarides JG. Preexcitation in a child with syncope: where is the connection? $J$ Cardiovase Electrophysiol $1998 ; 9 ; 892-895$

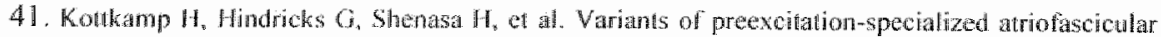
pathways, nodofascicuat pathways, and fasciculoventricular pathways: electrophysiologic dindings and target sites for radiofrequency catheter ablation. I Cardiovase Edectrophysiol $1996 ; 79: 60930$.

42. Strasberg $B$, Coelho $A$, Palleo $E$, et al: Pharnacological obserwations in patients with nodoventricular pathways. Br Hatr J. 1984,51:84-90.

43. Cappato $\mathrm{R}$, Schluter M. Weiss $\mathrm{C}$, et al: Catheter-induced mechanical conduction bfock of rightsided accessory fibers with Malaim-type preexcitation to guide radiofrequency ablation. Circuktion 1994:00:282-290.

44. Bothassen $B$, Viskin $S$, Fish $R$, el at: Caheter-induced mechanical trauma to accessory pathways during radiofrequency ablation: incidence, predictors and clinieal implications. $J$ Am Coll Cardio! $19099: 33: 767.774$. 


\section{Chapter 2}

\section{Differential diagnosis of left bundle-branch block-shaped tachycardias}

Eduardo Back Sternick ${ }^{*}$

Hein JJ Wellens ${ }^{\dagger}$

From the Biocor Instituto*, Belo Horizonte, Brazil, CARIM-

Cardiovascular Research Institute ${ }^{\dagger}$, Maastricht, The Netherlands 


\section{INTRODUCTION}

The correct diagnosis of the site of origin of a regular tachycardia with a wide QRS complex $(\geq 120$ msec) is of importance for management and prognosis of the patients. Over the last 40 years a number of approaches have been suggested to help the physician in this challenging decision making ${ }^{1-14}$. Intravenous medications erroneously given because of a misdiagnosis of SVT, particularly verapamil or diltazem, can be deleterious becanse they may precipitate hemodynamic collapse in the patient with VT. Stable vital signs during tachycardia are not helpful for distinguishing $\mathrm{SVT}$ from VT. If the diagnosis of SVT cannot be proven or cannot be made easily, then the patient should be treated as if VT were present ${ }^{15}$. The focus should be on the ECG signs which may not only help to distinguish between a VT from other tachycardias with a broad QRS complex but also suggest its etiology and site of origin in the ventricle.

\section{Classification of LBBB-shaped tachycardias:}

LBBB-shaped tachycardias can be divided into 3 groups (figure 1):

1. Supraventricular tachycardias with preexistent or functional LBBB

1.A. Pre-existent LBBB

1.B. Tachycardia-dependent phase 3 block

1.C. Retrograde invasion into the $\angle B B$

1.D. LBBB induced by drugs (pseudo VT)

2. Supraventricular tachycardias with AV conduction over a right sided accessory pathway

2.A. Rapidly conducting accessory pathways

2.B. Slowly conducting accessory (Mahaim) pathways

3. Monomorphic Ventricular tachycardia

3.A. Ectopic ventricular origin

3.B. Bundle branch reentry

\section{THE ECG DIAGNOSIS}

\section{AV dissociation}

Although dissociation between atrial and ventricular activity during tachycardia is a hallmark of VT (lead II, figure 2), some form of V-A conduction can be present during $V T$, particularly during a slow $V T$ (figure 3$)^{7}$. P waves can be difficult to recognize during a broad QRS tachycardia and it can be useful to look for nonelectrocardiographic signs such as variations in jugular pulsations, the loudness of the 
frrst heart sound and changes in systolic blood pressure, In patients with slow VT rates occasional conduction from atrium to ventricle over the $\mathrm{AV}$ node-bundle branch system may happen resulting in "capture"
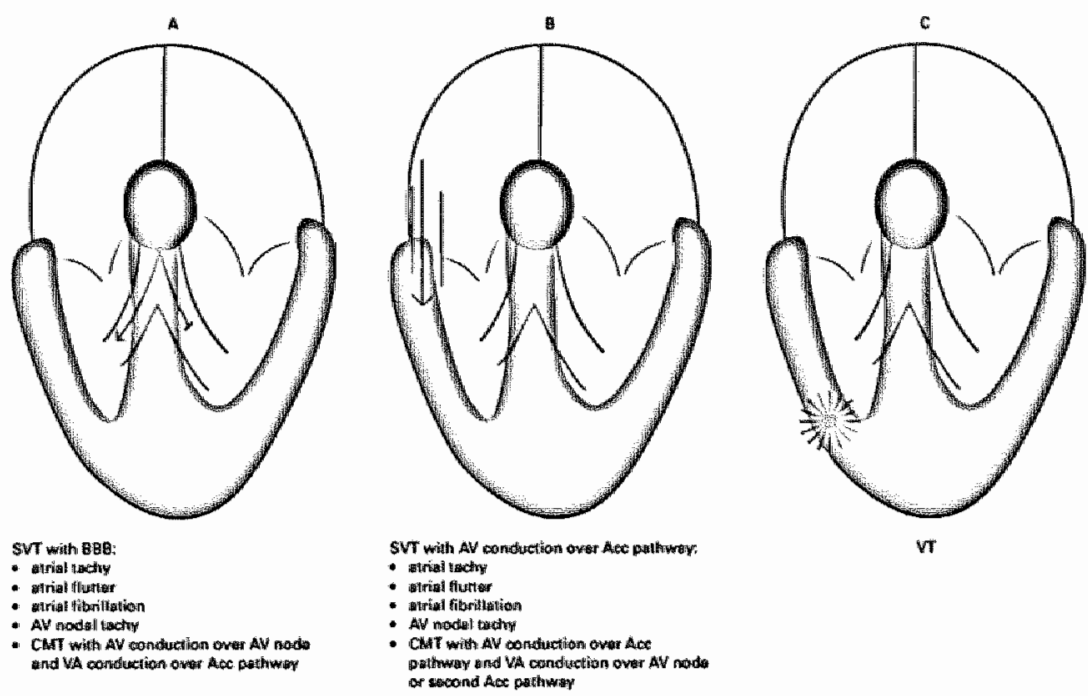

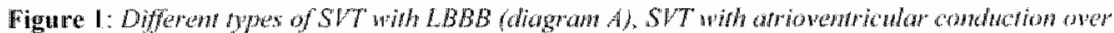

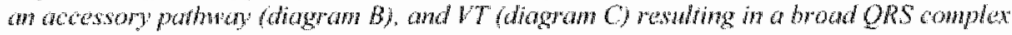
rerohyordia.

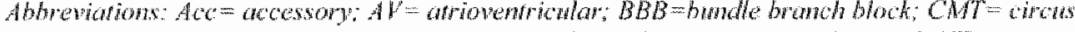

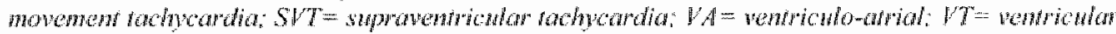
incriburatici.

or "fusion" beats. Sudden narrowing of a QRS complex during VT may also be the result of a premature ventricular depolarization arising in the ventricle in which the tachycardia originates, or it may occur when retrograde conduction during VT produces a ventricular echo beat leading to fusion with the VT QRS complex.

Very rarely AV dissociation is present in tachycardias other than VT. Ii may occur in A-V junctional tachycardia (JET) after cardiac surgery or during digitalis intoxication. A-V dissociation has been reported during narrow QRS complex tachycardia due to nodofascicular accessory pathway either manifest or concealed ${ }^{17,1 \%}$.

\section{Width of the QRS conplex}

As pointed out by Wellens and shown in figure 4 , the site of origin of the VT plays a role in the width of the QRS complex. When the arrhythmia arises far from the interwentricular septum, the sequential activation of the ventricles results in a very wide QRS. The QRS complex will be narrower when the VT has its origin in or close to the septum. Other factors like the size of the scar tissue (after myocardial infarction 
or myocarditis), ventricular hypertrophy, and muscular disarray (as in hypertrophic cardiomy opathy) also play a role in the QRS widh during VT.

It is of interest that a QRS widh of more than $0,16 \mathrm{sec}$ during $L B B B$ shaped VT argues for a $V T^{7}$. But a QRS widh below such value may occur in septal VTs. Of course, QRS width is not helpful in differentiating VT from a tachycardia with A-V
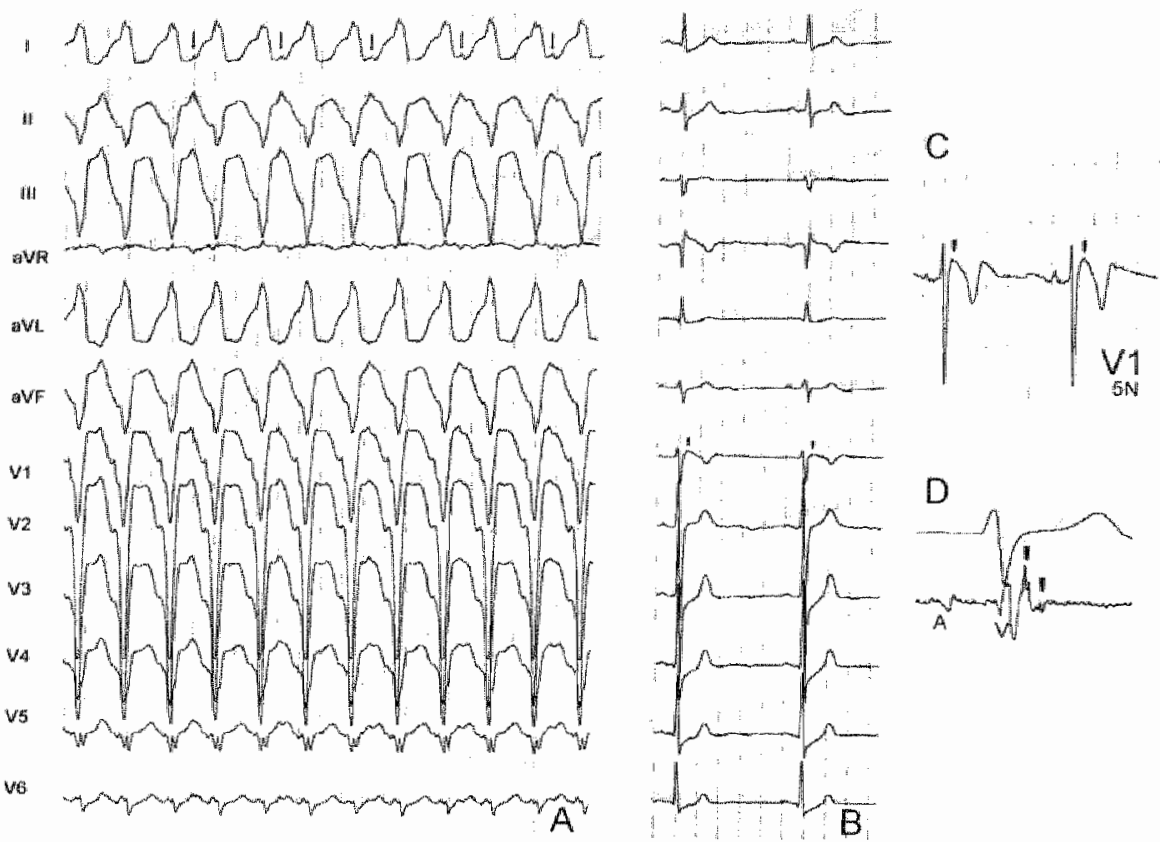

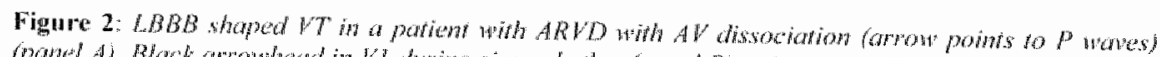

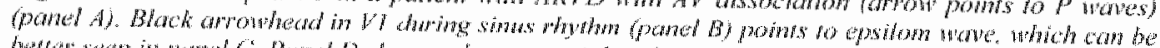

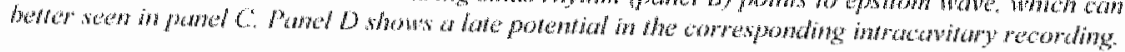

conduction over a tapidly conducting accessory pathway because such a pathway inserts into the ventricle leading to ecentric ventricular activation and w wide ORS complex (figure 5). QRS width it is also not helpful to distinguish between a SVT with LBBB from an antidromic tachycardia with anterograde conduction over a Mahaim fiber (figure 6). The Mahaim pathway can be a short AV structure inserting close to the annulus or a long fiber inserting at or close to the distal RBB. Tachycardias with anterograde conduction over" a short Mahaim usually show a QRS width larger than 0.16 seconds. However, the majority of atriofascicular Mahaim antidromic tachycardias show a QRS complex in between 0,12 and 0.14 seconds. Other distinctive findings ${ }^{20}$ include an $R$ wave in lead 1, rS in $V_{1}$, an $R / S>1$ precordial transition after $V_{4}$, and a ORS axis to the left of -30 . An SVT with LBBB 
can have a QRS width of more than 0,16 under three circumctances: (1) in the presence of pre-existent $\angle B B B$ in the elderly with fibrosis in the bunde branch system and ventricular myocardium (figure 7); (2) when during SVT AV conduction occurs over an accessory pathway: (3) when class IC drugs (specially tlecainide) are present during SVT (figure 8).
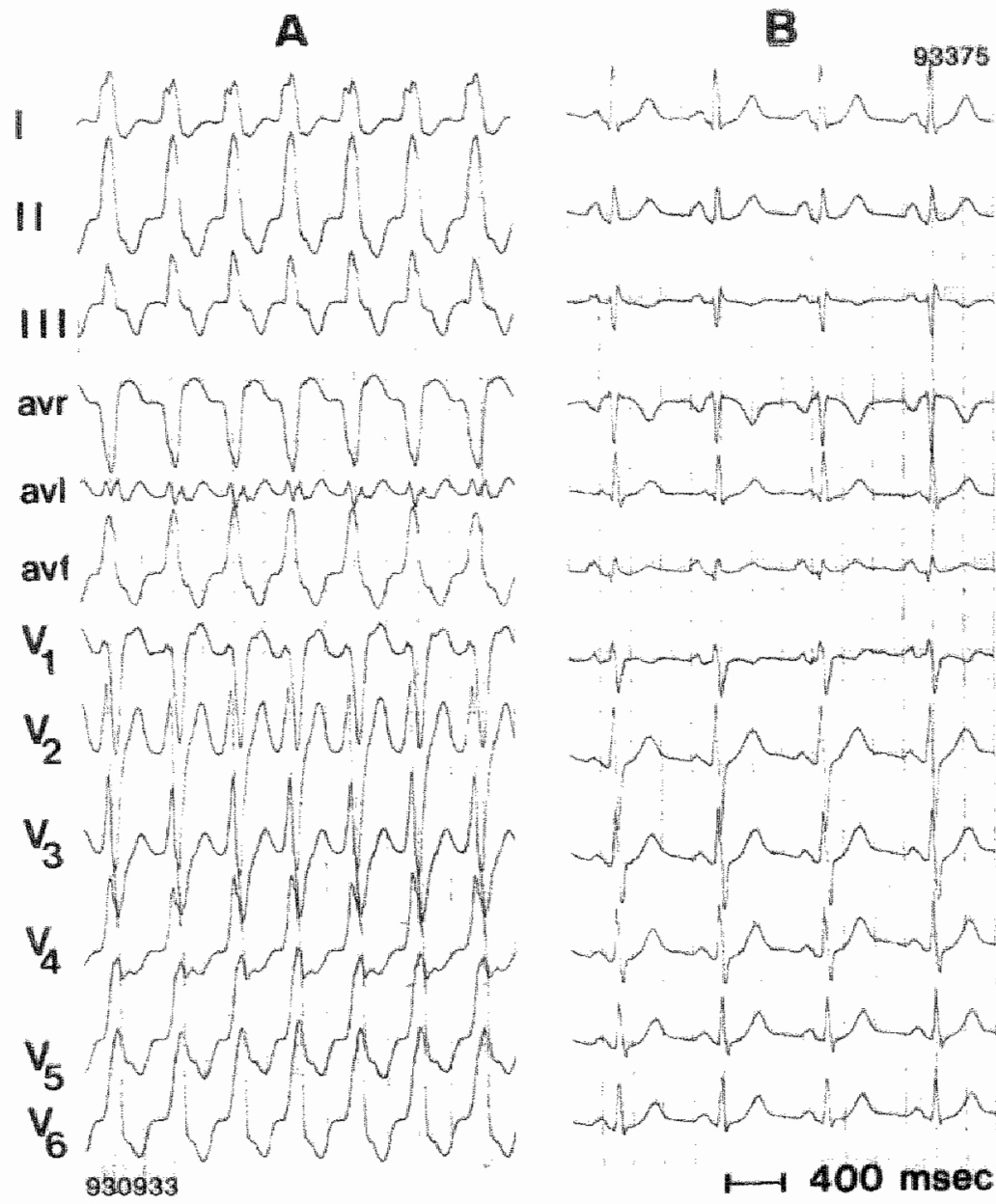

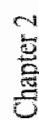
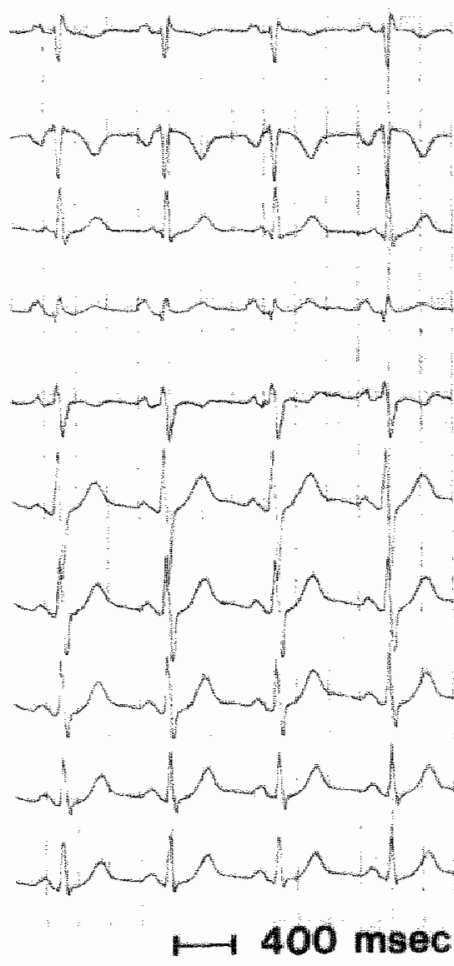

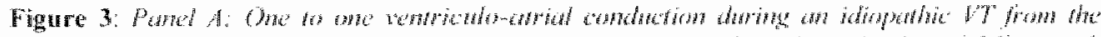

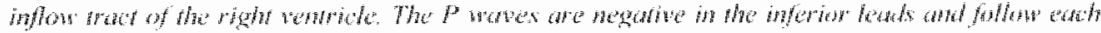

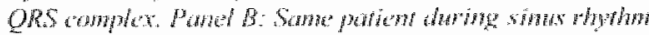


VT origin close to Interventricular septum - more stimultaneous ventricular activation — - more narrow QRS

I

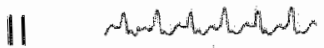

III Whowht

$\frac{5}{3}$

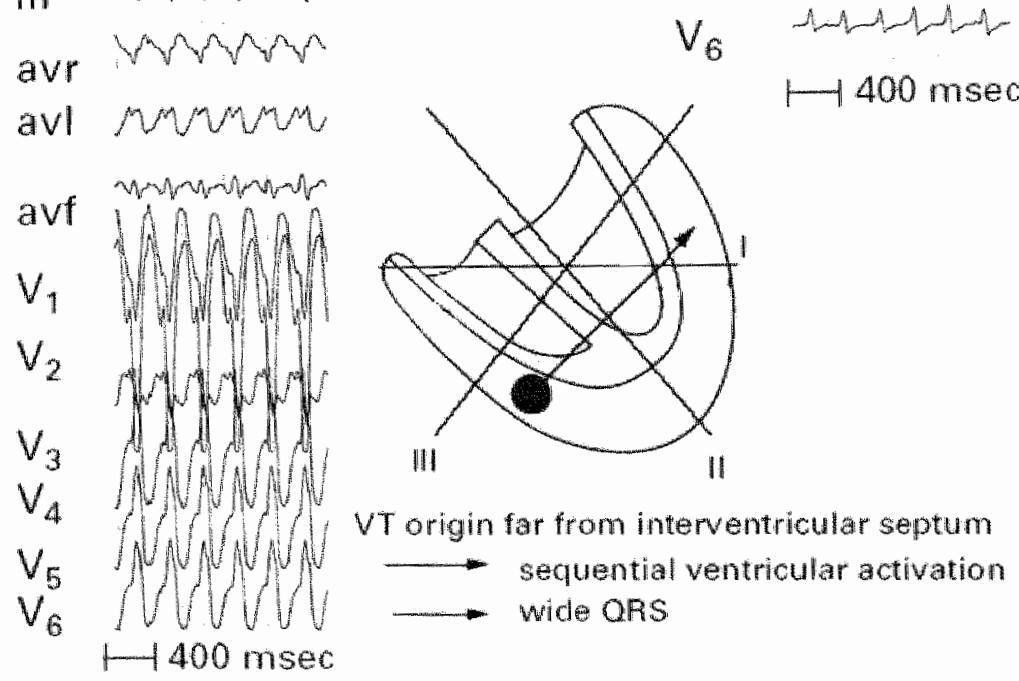

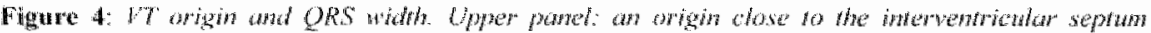

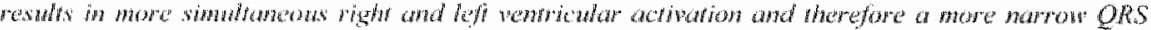

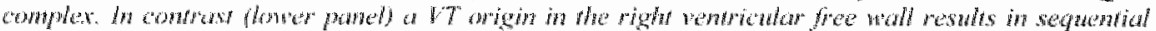

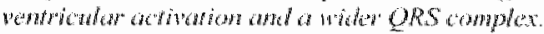




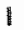

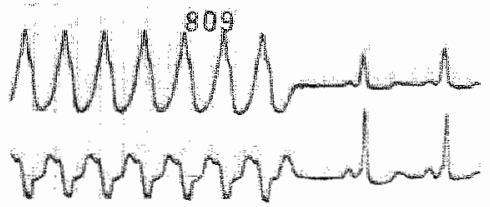

III

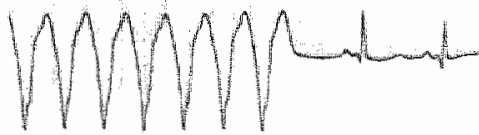

R

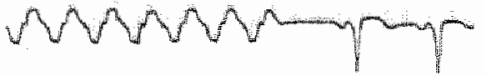

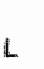

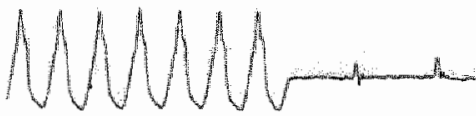

$\mathbb{F}$

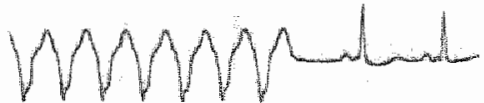

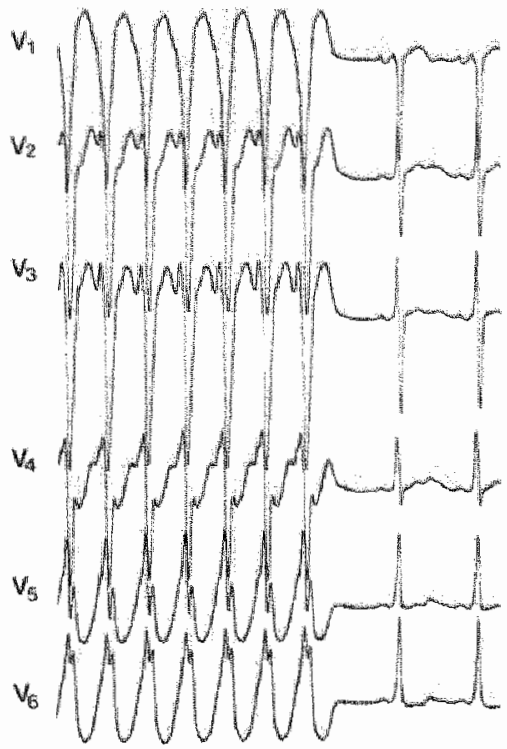

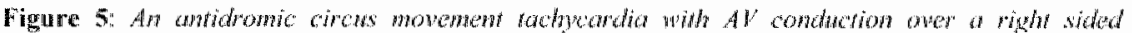

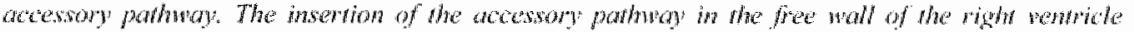

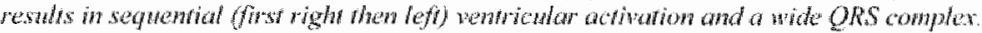

\section{QRS axis in the frontal plane}

The QRS axis is not only important for the differentiation of the broad QRS tachycardia but also to identify its site of origin and etiology. A VT origin in the apico-septal region of the LV has a superior axis (to the left of $\left.-30^{\circ}\right)$. LBBB with an inferior axis occurs with VT from the right ventricular outflow tract or at the pulmonary artery, just above the pulmonic valve.

\section{Configurational characteristics of the QRS complex}

\section{Leodis VI and $V 6$}

In LBBB shaped VT, lead $V_{1}$ (and also $V_{2}$ ) (figure 9) usually shows: an initially positive QRS with positivity measuring more than 0,03 seconds; slurring or notching of the downstroke of the $S$ wave; and an interval between the beginning of the QRS axis and the nadir of the $S$ wave of 0,07 seconds or more ${ }^{\text {lfit }}$. When lead $V_{6}$ shows a qR pattern during LBBB shaped tachycardia, VT is very likely. In SVT with LBBB, lead $V_{1}$ shows no or minimal initial positivity, a very rapid downstroke of the $\mathbf{S}$ wave, and a short interval between the beginning of the QRS and the nadir of the $\mathbf{S}$ wave (figure 10). 


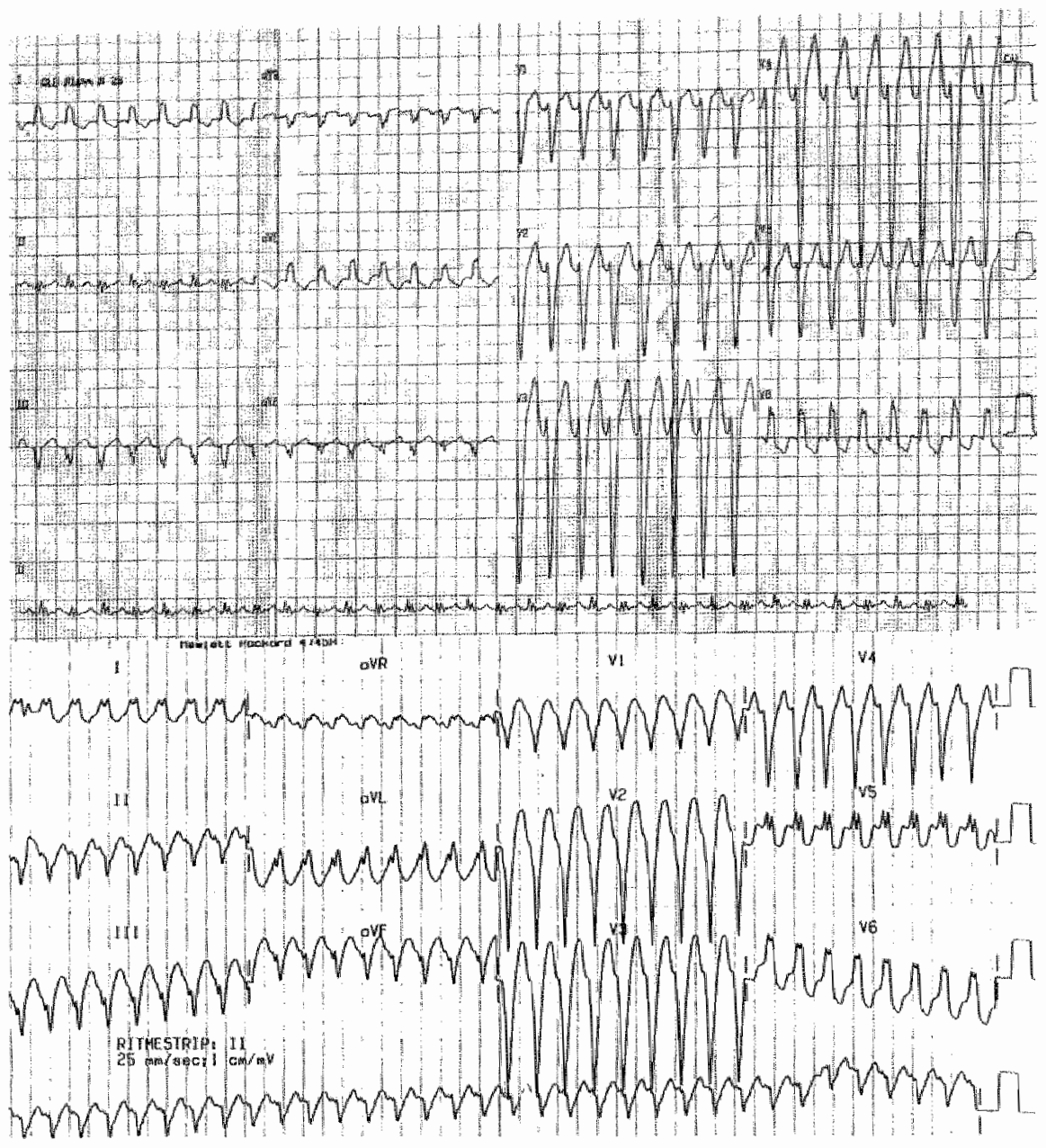

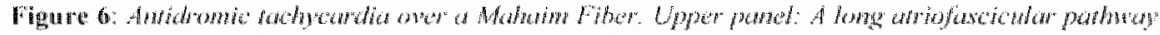

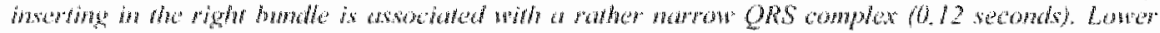

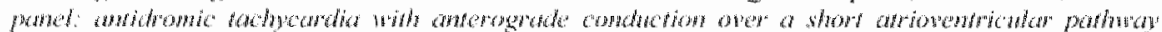

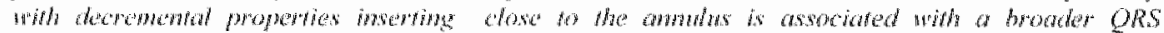

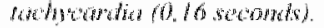




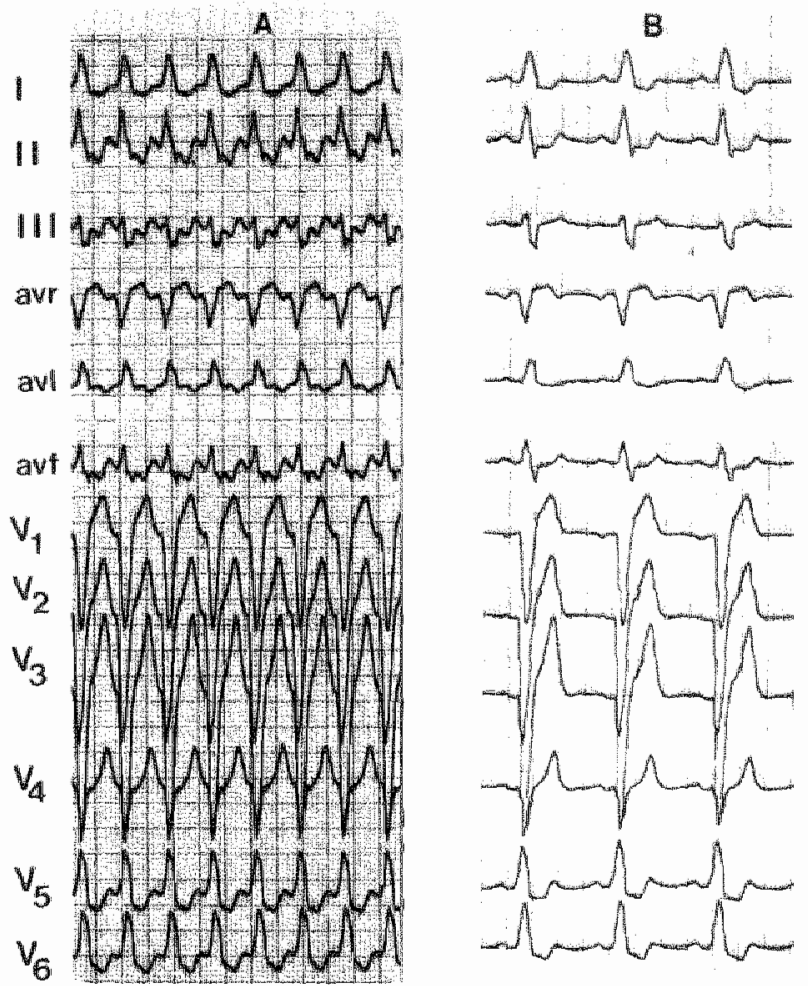

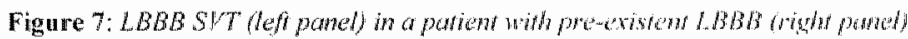

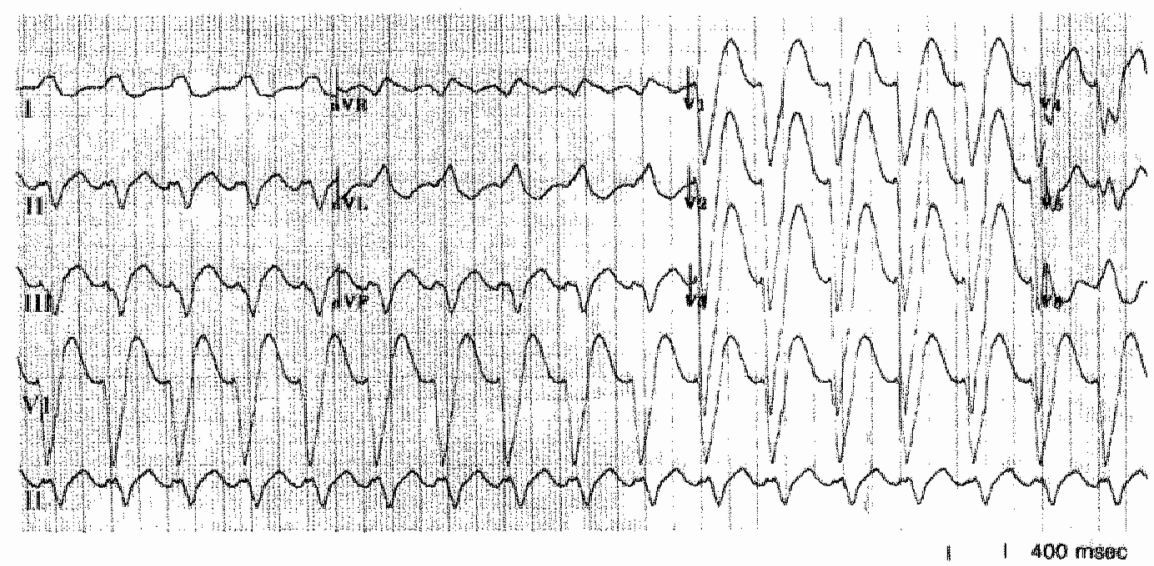

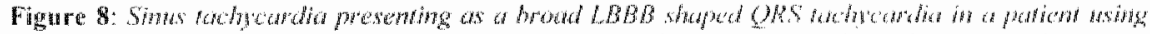

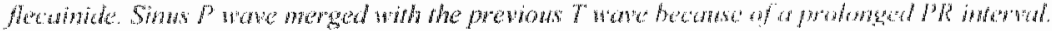



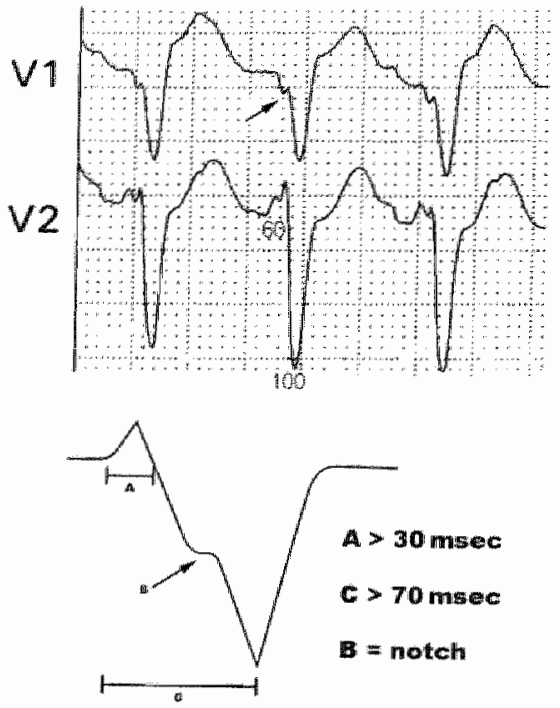

Figure 9: Upper panel: Wat and 12 from a parient with $V T$ showing an hidol to wave widh of 60 miste, a notch ar the downtroke ing and an thremal between the beginghing of the QRS to the nadig of the $S$ wher of wor wase.

Lower pare: Findings in lead WI and 12 dyring $\angle B B B$ shaped tachycardia powning to a veviriculare osigh.

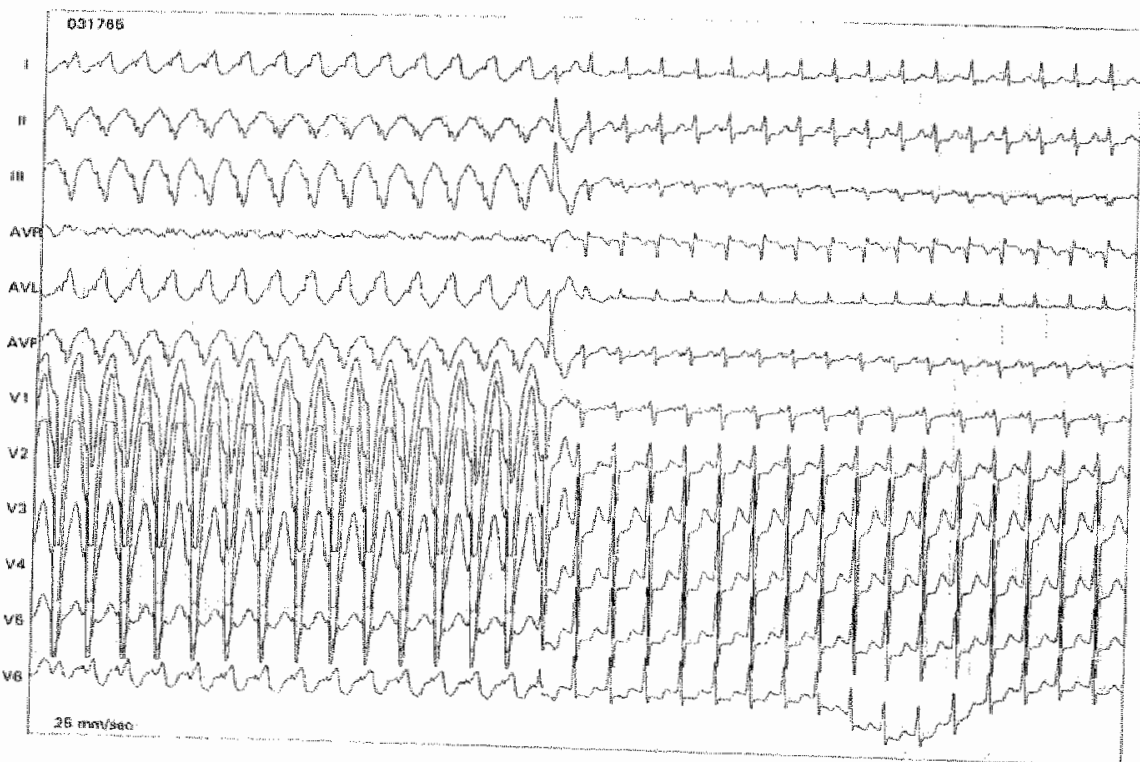

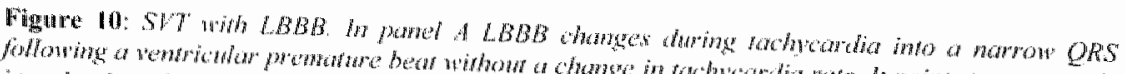

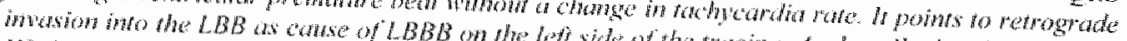

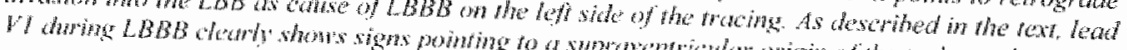



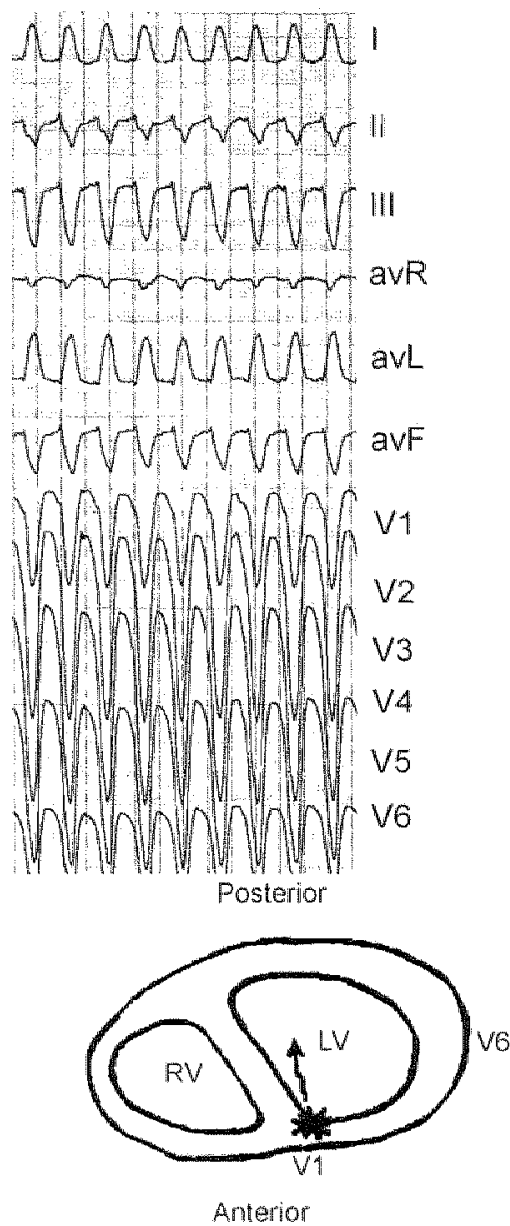

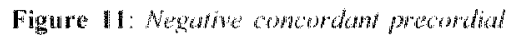

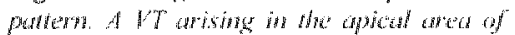

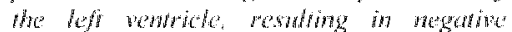

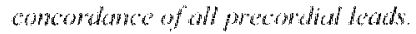

LBBB shaped VTS associated with coronary antery disease are usually adjacent to the inter-ventricular septum and have a higher predictive accuracy for their site of origin than RBBB shaped VTs. The ECG from a $\angle B B B$ VT originating in a scar at the apicomseptal region will show $\mathrm{Q}$ wave in leads $I$ and $\mathrm{V}_{6}{ }^{21}$.

\section{Interwal onset QRS to madir of $\mathrm{S}$} wave in precordial leads

Brugada et at ${ }^{12}$ suggested that an $\mathbb{R} S$ interval $>100 \mathrm{~ms}$ in one or more precondialleads is highly suggestive for VT. One should be careful, howewer, because such a number may occur in $S V T$ with $A \cdot V$ conduction over an accessory pathway, SVT during administration of drugs that slow intraventricular conduction and in SVT with preexistent BBB, especially $\angle B B B$.

\section{Concordant pattern}

When all precondial leads show either negative or positive ORS complexes this is called negative or positive precordial conoordance. Negative concordance is diagnostic for a VT arising in the apical area of the heart (figure 11). However, an abnormal anatomic position of the heart in a patient with SVT with left bundle branch block can lead to negative concordance in the precondial leads (figure 12) and a false diagnosis of ventricular tachycardia. One example is pechs excavarm where the right ventricle lies completely under the anterolateral precordial area ${ }^{22}$. Positive concordanee (all QRS complexes in the precordial leads being positive) can oceur in a VT arising in the posterior part of the left ventricle or in lachycardia with $A-V$ conduction over a left posterior accessory pathway. 


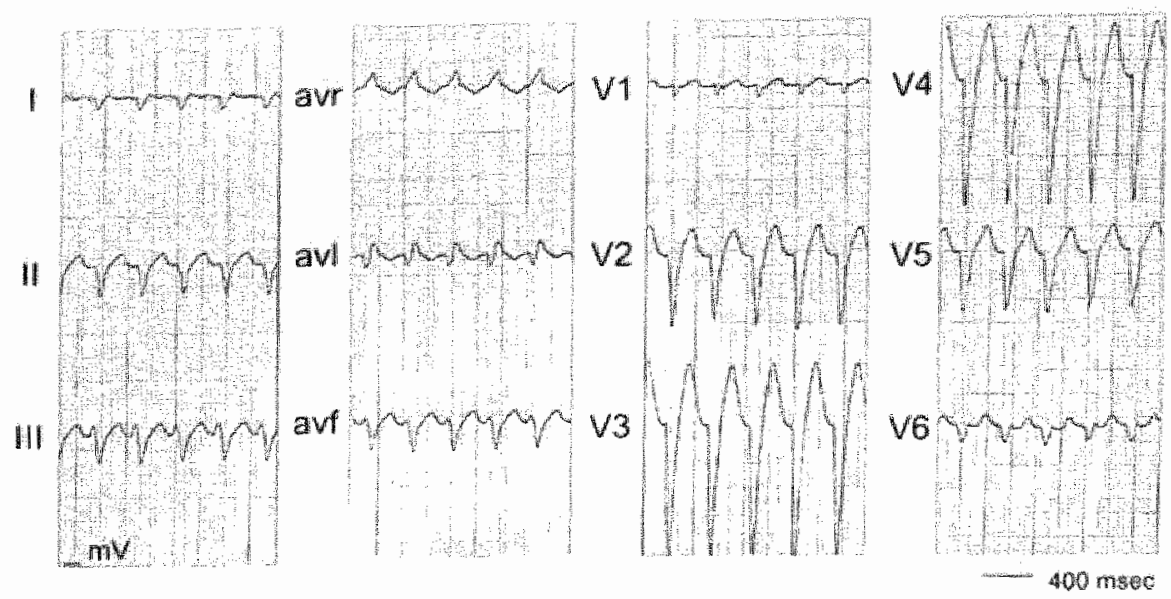

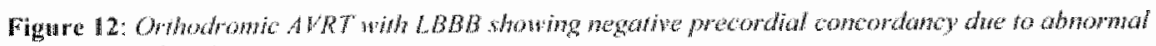

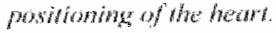

\section{Tachycardia QRS identical to the sinus $Q R S$}

When the broad QRS is identical during tachycardia and sinus rhythm, one has to difrerentiate SVT with pre-existent LBBB (figure 7) from bundle branch re-entrant tachycardia. In diseased hearts, especially when the bundle branches and the interventricular septum are involved, a tachycardia may occur based upon a circuit with anterograde conduction dow one bundle branch and after septal activation retrograde conduction over another branch of the bundle branch system (ligure 13). This type of re-entry may occur in patients with anteroseptal myocardial infarction, idiopathic dilated cardiomyopathy, myotonic dystrophy, after aortic valve surgery, and after severe frontal chest trauma.

\section{Tachycardia QRS more narrow than sinus QRS}

When during tachycardia the QRS is nanower than the one during sinus thythm a $V T$ should be diagnosed. This can be explained by the site of origin of the VT close to the inter-ventricular septum, resulting in more simultaneous activation of the ventricles in contrast to the sequential activation of first the right and then the left ventricle in the presence of $\angle B B B$ during sinus thythm.

\section{Presence of $Q R$ complexes}

Coumel et $a^{2}$ called attention to the significance of a QR (but not a QS) complex during a broad QRS tachycardia, showing that their presence indicates a scar in the myocardium usually caused by myocardial infarction. Figure 14 gives an example of QR complexes during a $L B B B$ shaped $V T$ in a patient with an old inferior myocardial infarction. QR or qR complexes during VT are present in approximately $40 \%$ of VTs after myocardial infarction ${ }^{25}$. 


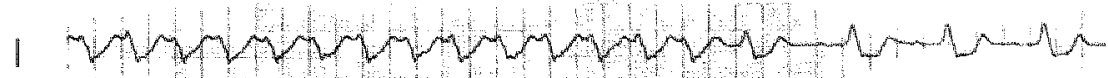

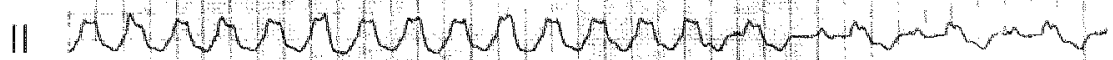

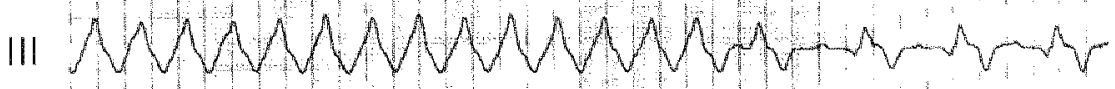

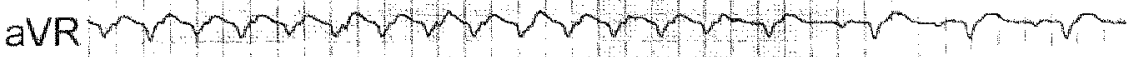

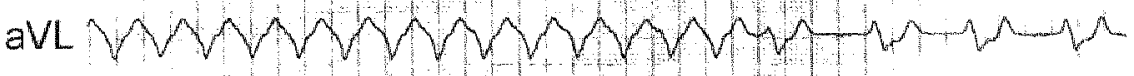

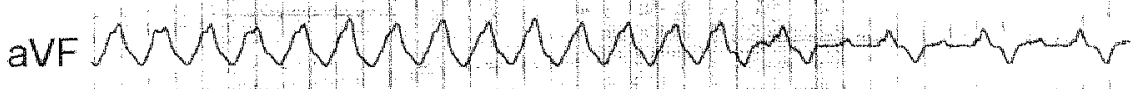

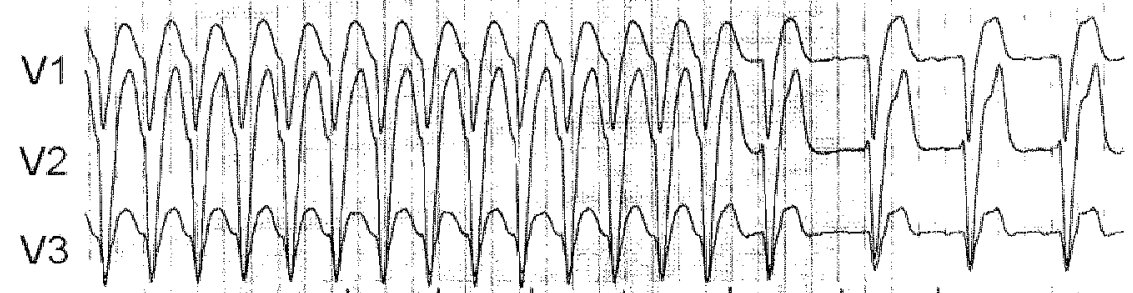
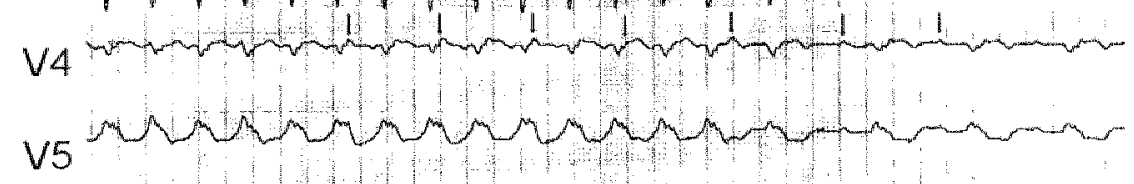

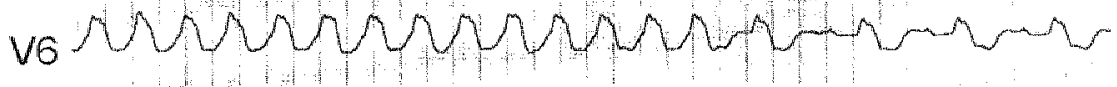

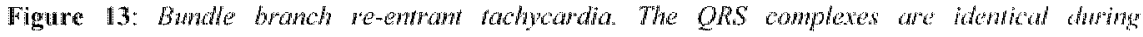

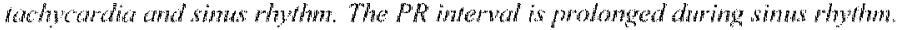



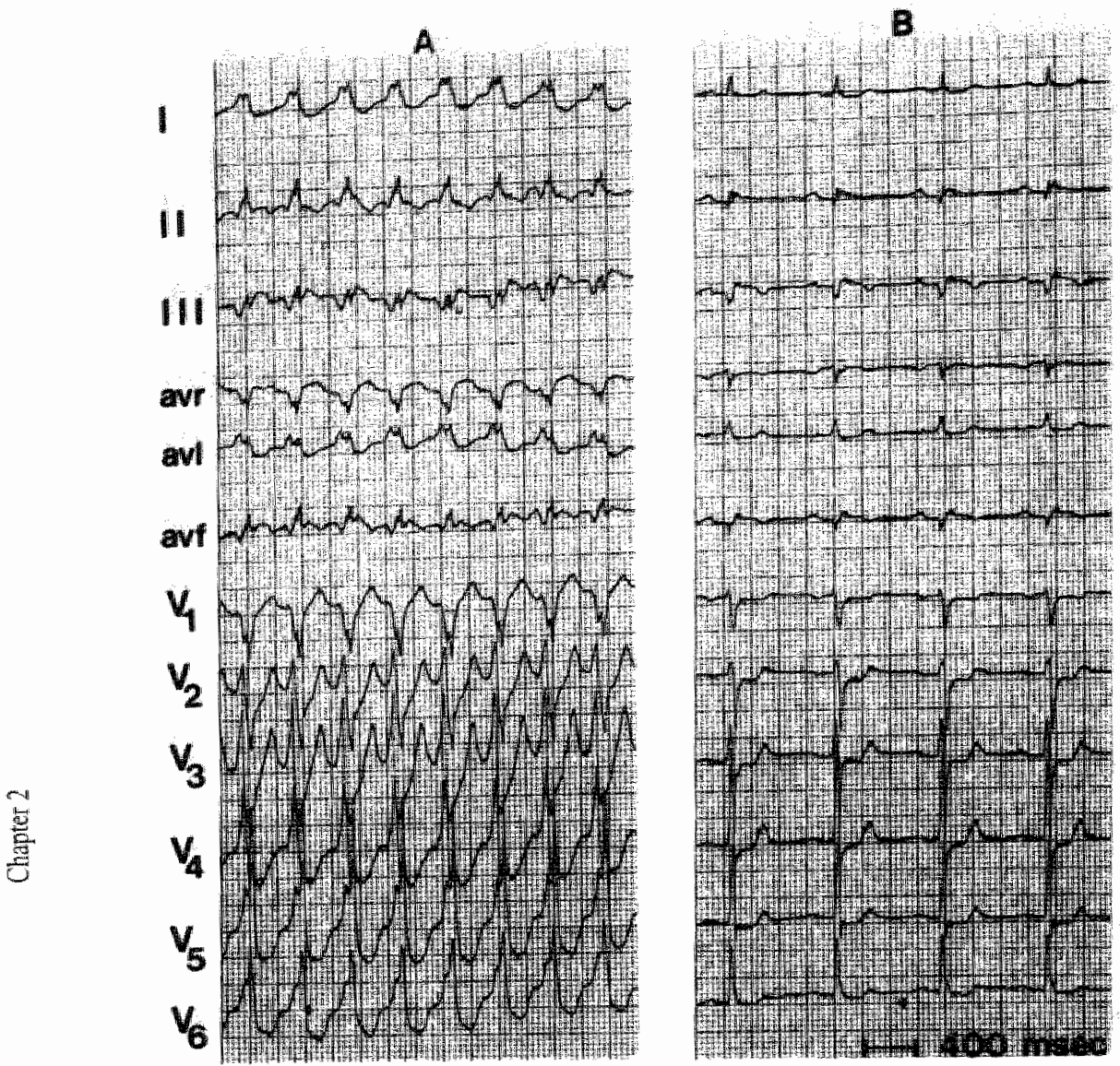

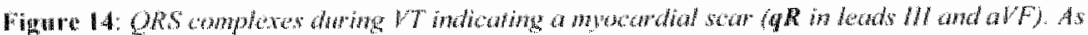

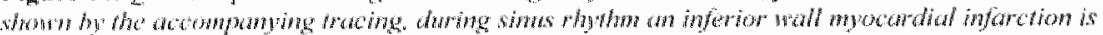
pwastom.

\section{Ethology of VT}

Most VTs have a previous myocardial infaretion as their etiology, and, as pointed out, a OR complex during VT can be wery helpful to make that diagnosis.

However, characteristic ECG patterns can also be found in idopathic VT ${ }^{20}$ and $\mathrm{VT}$ in patients with arrbythmogenic right ventricular dysplasia (ARVD) ${ }^{27}$. Figure 15 shows the QRS pattern of an idiopathic VT arising close to the outflow tract of the right ventricle.

In some patients tachycardia does not arise on the endocardial surface of the rightventricular outllow tract but epicardially around the root of the pulmonary valwe $e^{2 k}$, in the root of the aorta going to the posterior part of the outllow tract of the right ventricle. An early precordial transition $(\mathrm{R} / \mathrm{S}>1$ ) and or a greater wave in right precondial leads is consistent with an epicardial site of origin ${ }^{29}$. 


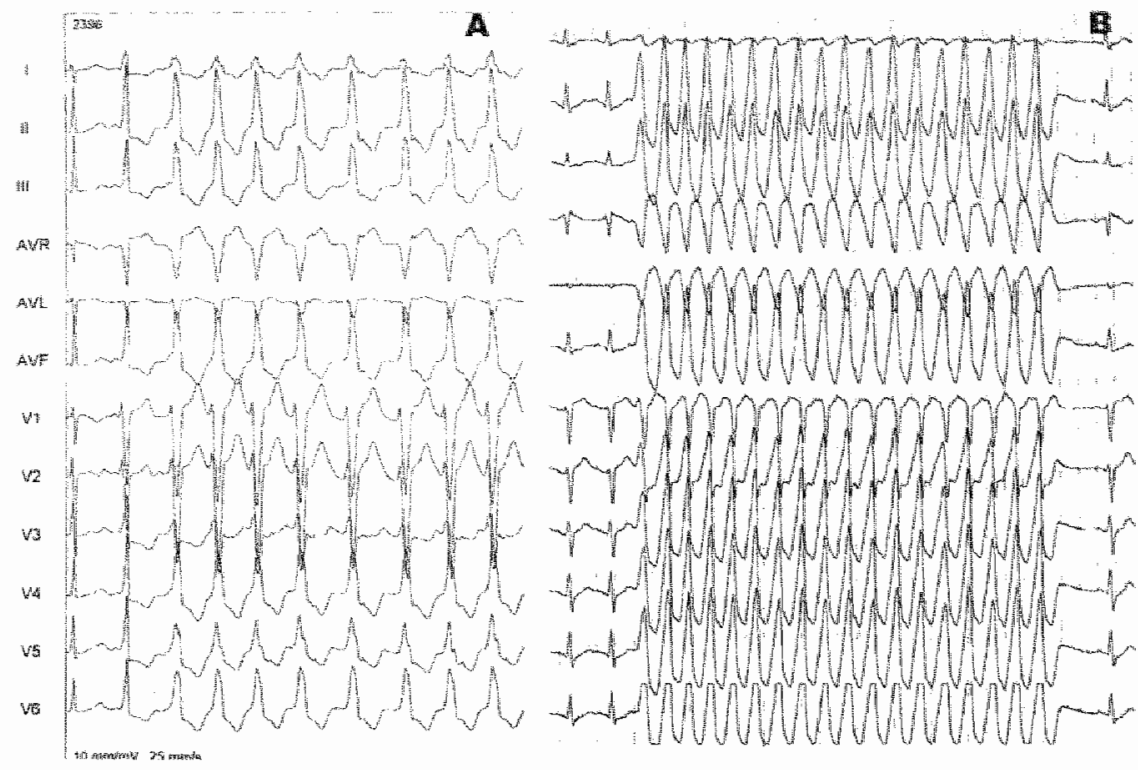

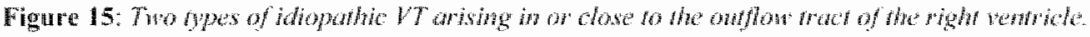

In ARVD there are three predilection sites in the right ventricle: the inflow and outfow tract, and the apex (figure 16). While the first two sites have a QRS configuration during tachycardia which is difficult to differentiate from right ventricular idiopathic VT, left axis deviation in a young person with an LBBB shaped VT should immediately lead to the suspicion of ARVD (figure 2 panel A). In fact, there is an important rule in $\angle B B B B$ shaped VT with left axis deviation that cardiac disease should be suspected and that idiopathic right ventricular VT is unlikely.

\section{Value of the $\mathrm{ECG}$ during sinus rhythm}

The ECG during sinus rhythm may show changes such as pre-existent 1 BBB, ventricular premexcitation, or an old myocardial infaretion, which are very helpful in correctly interpreting the $\mathbb{E C G}$ during a $L B B B$ shaped QRS tachycardia. We llave recently described ${ }^{30}$ a nowel ECG signal suggestive of a Mahaim fiber in young patients with palpitations, which is an $\mathrm{IS}$ pattem in lead III. This pattem can be seen in $2 \%$ of normal young persons but when this $\mathrm{rS}$ pattern is associated with the absence of a $q$ wave in lead $I$ it is wery specific for Mahaim fibers. The presence of $A$ $V$ conduction dishrbances during sinus rhythm make it very unlikely that a broad QRS tachycardia in that patient has a supraventricular origm and, as discussed, a QRS width during tachycardia narrower than during sinus thythm points to a VT. 


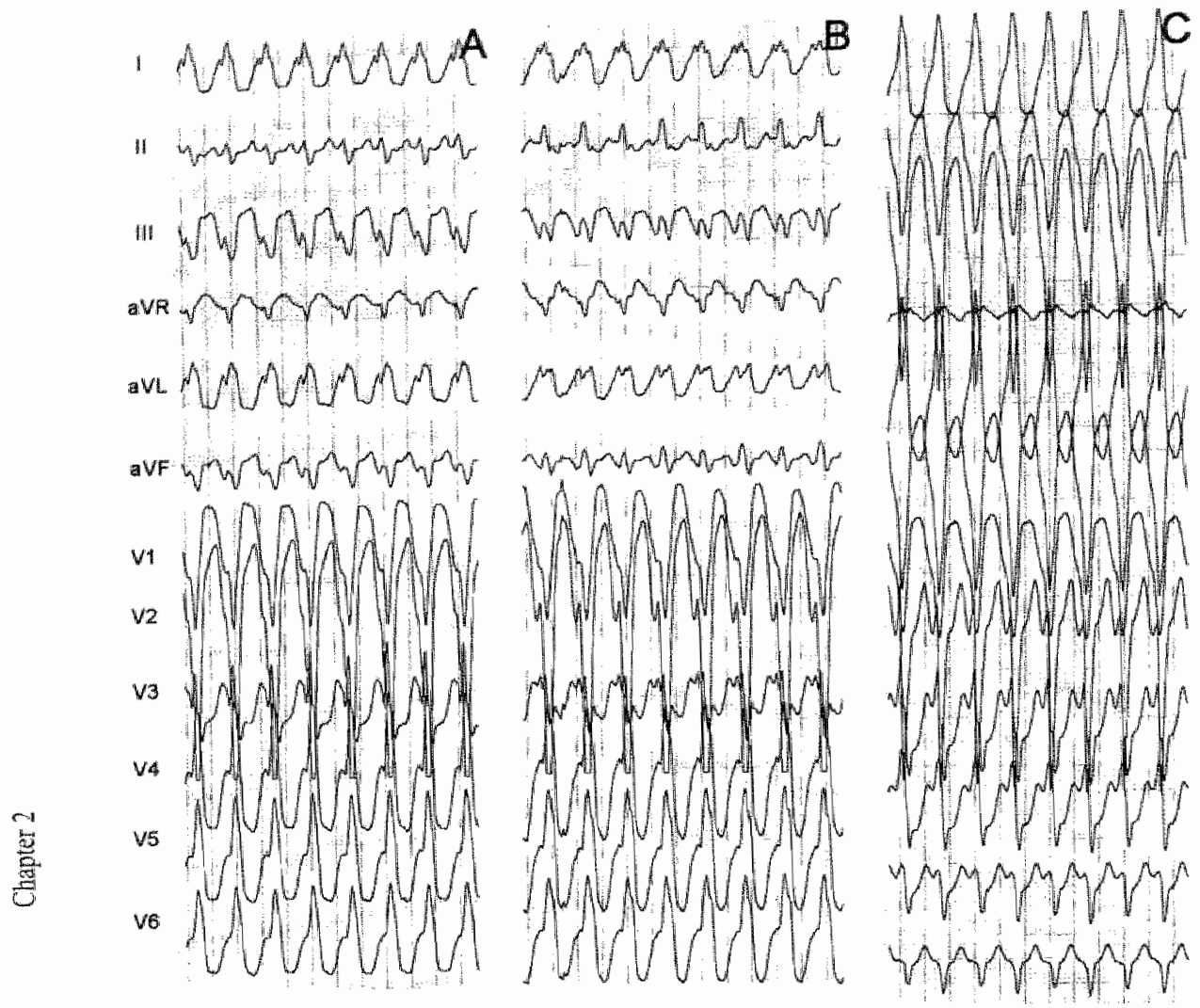

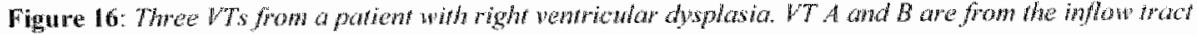
whine vT on panel $C$ originares from the aper.

\section{References}

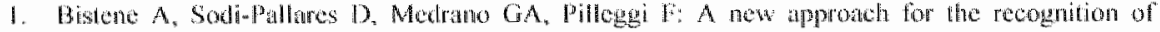

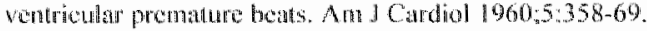

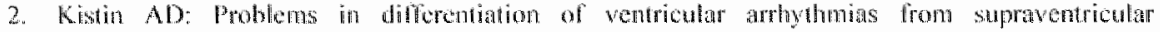

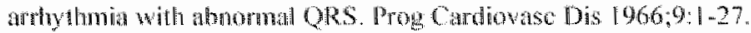

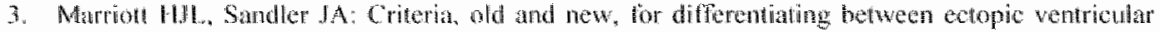

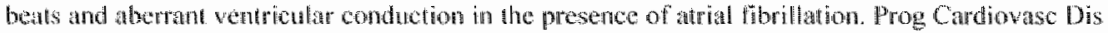
$1966: 9: 18-28$.

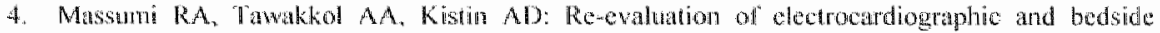

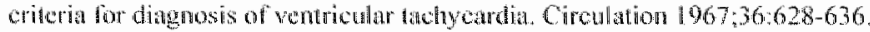

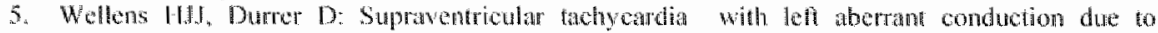
retrogradis invasion into the fef bundle branch, Circulation 1968:38:474-479. 


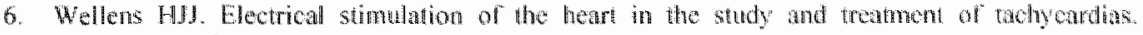
University Pank Press, Baltimote. 1971

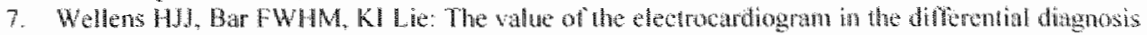

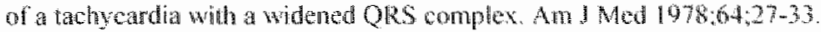

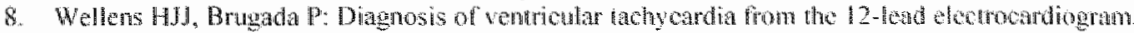
Candiology Clinics 1987,5:511-525.

9. Dongas 1, Lehmann MIL. Mathud R, et al: Walue of pe-existing bundle banch block in th: electrocardiographic diferentiation of supraventricular from mentricular origin of wide QRS atchycarda. And I Candiol $1985 ; 55: 717-21$.

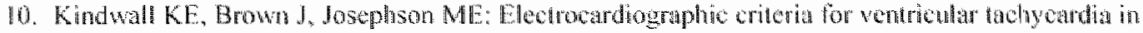

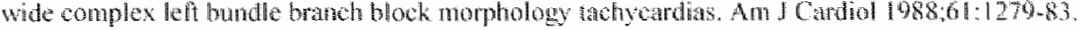

11. Grifhly MJ de Belder MA. Linker NJ, of al: Multivariate analysis to simplily dillerentint

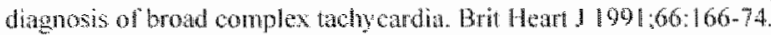

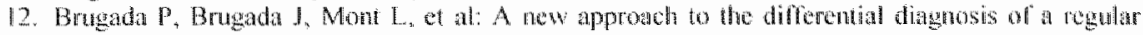

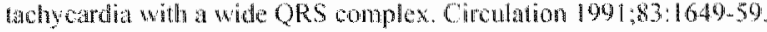

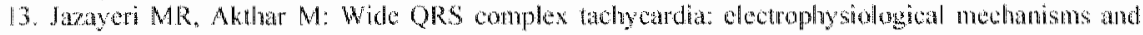

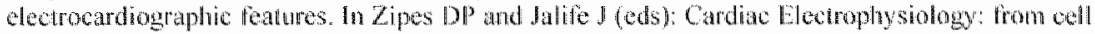
to hedside. Philadelpha, WB Satuders Co, 1994, p990.

14. Nibloy C. Whaton IM: Ventricular tachycardias with lell bunde branch block morphology. Waing Gin Eicetrophysial. 1995; 18:334-50.

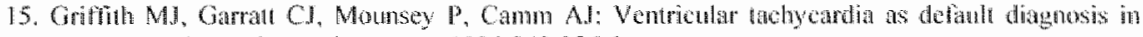
broad complex acliycardia. Lancel $1994 ; 343: 386-8$.

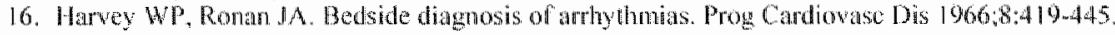

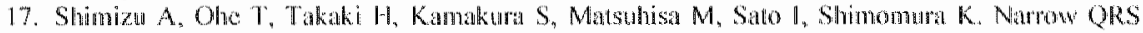
complex lachyordia with atroventricular dissociation: Pacing Clin Floctrophysiol 1988:1 1:384393.

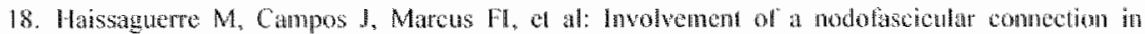

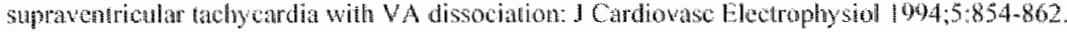

19. Wellens tIfH, Wentricular Hehyeardia: diagnosis of broad ORS complex tachycardia. Hean $2001 ; 86: 579-585$.

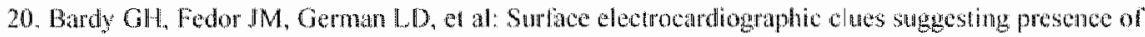
a nodofascicular mahain nber. I An Coll Candiol 1984;:1161-8.

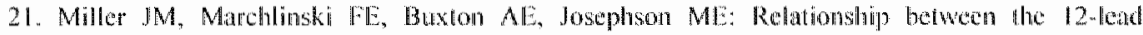
elcchocardiogran duning VT and endocardal site of origin in patents with coronary artery discase. Circulation $1988 ; 77: 759-76$ ?

22. Volders PGA. Timmermans C, Rodriguez LM, et al: Wide QRS complex lachyoardia with

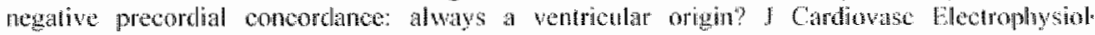
$2003: 14: 100-111$.

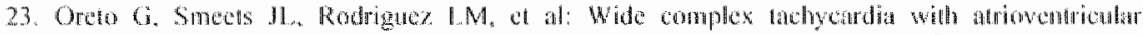

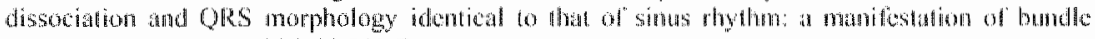
bramele reantry. Heare 1996:76:54:-7.

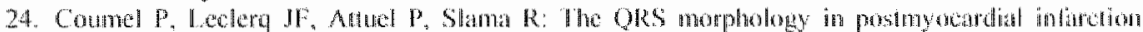

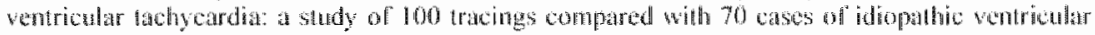

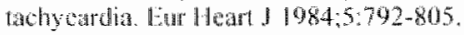

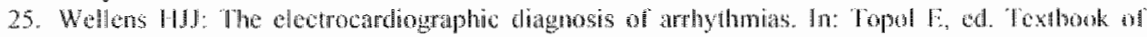

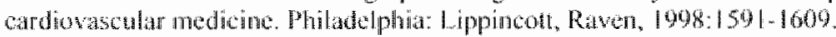

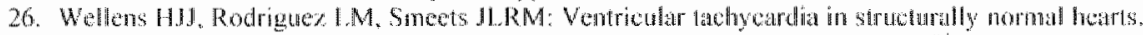

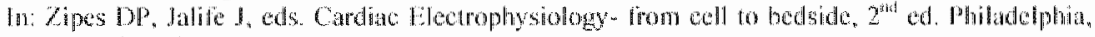
WB Saunders, 1995:780-8.

27. Leterq JP. Cound PH: Chanderistics, prognosis and tretment of the ventricular armythmias of

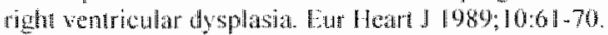

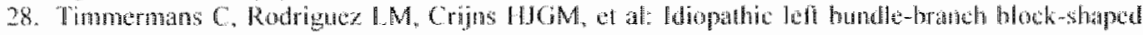

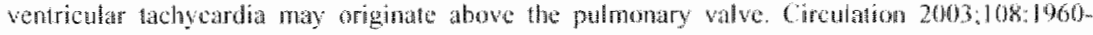
1967. 
29. Ouyang $F$, Foluhi $P$, Ho Sy, al al. Repetrive monomorphic venticular tachycardia origunating from the arotic sinus cusp: ellectrocadiographic characterization for guding catheter ablation. I Am Coll Cardiol 2002;39500-8.

30. Silemick EB, Thmormans C, Sosa E, at al: The electrocardiogram in sinus rhy thrn and during tachycardiat in patients wh anterogeade conduction over Mallaim fibers: The role of the " $\mathrm{t} S$ " pattern in lead III. I Am Coll Cardiol 200:4:44: 1626-1635. 


\section{Chapter 3}

\section{The electrocardiogram during sinus rhythm and tachycardia in patients with Mahaim fibers. The importance of an " $r S$ " pattern in lead III}

Eduardo Back Sternick*

Carl Timmermans ${ }^{\dagger}$

Eduardo A. Sosa $\ddagger$

Fernando E. S. Cruz Filho

Luz-Maria Rodriguez $^{\dagger}$

Luiz M. Gerken*

Márcio L. A. Fagundes

Hein J.J. Wellenst

From the Biocor Instituto*, Belo Horizonte, Brazil, University Hospitalt, Maastricht, The Netherlands, Instituto do Coração da $^{2}$ Faculdade de Medicina da Universidade de São Paulo, Brazil, Instituto Nacional de Cardiologia Laranjeiras, Ministério da Saúde", Rio de Janeiro, Brazil.

Journal of the American College of Cardiology 2004;44:1626-1635 


\begin{abstract}
Background: Mahaim fabers are siowly conducting accessory pathways reaching into the right ventricle. They often play a role in tachycardias. The purpose of the study was to identify the electrocardiographic characteristics of the Mahaim fiber.

Methods and Results: We refrospectively analyzed 40 patients with Mahaim fibers. Five patients had associated WPW, and were excluded from the study. Two patients had a short atrioventricular decremental accessory pathways and were also excluded. The remaining 33 patients had a tachycardia with anterograde conduction over a Mahaim fiber. "Twenty were female. Mean age was $24 \pm 10$ years. The most common patten of minimal preexcitation during sinus thy thm was an $\mathrm{rS}$ pattern in lead III. This was found in 20 patients. There was a match between the presence of $\mathbf{r S}$ in lead III durng sinus thythm and left axis deviation during tachycardia with anterograde conduction over the Mahaim fiber. After ablation a different QRS pattern emerged in lead III, indicating absence of conduction over the Mahaim fiber. To obtain information about the prevalence of an $\mathrm{YS}$ pattern in fead III in age matched controls with palpitations and without structural heart disease, the 12-lead ECG of 200 young individuals were examined. An $\mathrm{rS}$ pattern in lead Ill was found in $6 \%$.

Conclusion: A narrow QRS with an $\mathrm{rS}$ pattem in lead Ill during sinus rhythm in a patient witl a history of palpitations should alen the physician to the possibility of a Mahaim fiber. During tachycardia these paticnts typically show a left bundle branch block like QRS complex with lefi axis deviation.
\end{abstract}




\section{INTRODUCTION}

Accessory pathways with long and decremental anterograde conduction thave been the subject of extensive debate about their anatomic structure ${ }^{1-4}$, location" "related

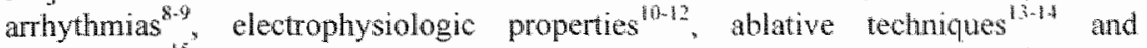
automaticity $^{15}$. Less attention has been given to the 12 -lead $\mathbb{E C O}$, especially to the ECG during sinus rhythm. The latter is considered to be normal in the majority of patients with atriofascicular pathways and patients with long atrioventricular decrementally conducting accessory pathways. Minimal preexcitation is reported to occur from 0 to $30 \%^{13.44}$. $16-17$, and apart from the absence of $q$ waves in the left precordial leads ${ }^{\text {* }}$ no specific QRS pattem has been described. The purpose of this article is to report on ECG findings in a large series of patients with Mahaim during sinus thythm and tachycardia, with emphasis on a particular ECG pattern in lead III during sinus thythm.

\section{STUDY POPULATION AND METHODS}

We retrospectively analysed 12 -lead ECG's from 40 patients having anterograde conduction over accessory pathways with long conduction times and decremental properties both during simus thythm, and during tachycardia. Five patients also had anterogradelly rapidly conducting accessory pathways and were excluded from the study. Two patients with a short atrioventricular deeremental pathway were also excluded from this series.

Definition of terms: we use the eponym Mahaim fibers in this study for an anterogradely conducting accessory $\mathrm{A}-\mathrm{V}$ pathway with long and decremental properties having a long anatomic course: either atriofascicular pathways ( 30 patients) or atrioventricular pathways ( 3 patients).

Every one of these 33 patients showed ellectrophysiologic criteria for a decrementally conducting bypass tract during atrial pacing, such as a progressive $\mathrm{AH}$ and $\mathrm{A}-\mathrm{V}$ interval prolongation coupled with a decreasing HV interval leading to a greater degree of pre-excitation with a left bundle branch block-like morphology 3,810 .

There were 20 fomales and 13 males, with a mean age of $24 \pm 10$ (range 8 to 52 ) years. All patients were referred for electrophysiologic assessment of a preexciled tachycardia. Preexcited AVNRT using a Mahaim fiber as a bystander was present in 1 patient. One patient had atrial fibrillation with prexcited QRS complexes and 2 patients were referred because of repetitive episodes of unsustained tachycardia caused by automaticity arising in the Mahaim fiber" (table 1). Ebstein's disease was diagnosed in 4 patients. The atrial insertion of the Mahaim fiber was located by the recording of a discrete accessory pathway potential in 28 patients and in 5 paticnts by assessing the shortest $\mathrm{A}-\mathrm{V}$ interval during atrial pacing at different sites along the tricuspid annulus. All patients underwent successful surgical $(n=2)$ or radiofrequency catheter ablation $(n=31)$. $R F$ ablation was guided by discrete potentials at the tricuspid annulus (28 patients) and by right ventricular pacemapping in 5 patients. 
We also analyzed the 12-lead ECG during sinus thythm in 200 individuals with palpitations and whout structural heart disease matched for age and gender as a control group.

Recordings of the 12-lead surface ECG and intracardiac electrograms were made using the EP Tracer or MS System (CardioTek BV, Maastricht, The Netherlands).

\section{Definitions of the QRS patterns}

ECGs were examined by two different observers with a magnified lens and a third observer decided when there was a mismatch classification. The following QRS patterns were found: $r / \mathrm{rS} / \mathrm{RS} / \mathrm{Rs} / \mathrm{rsR} / \mathrm{rs}^{*} / \mathrm{R} / \mathrm{qR} / \mathrm{QR} / \mathrm{QS} / \mathrm{qRs} / \mathrm{qRS}$. Very low woltage QRS complex $(<0,3$ mv) were depicted as small letters $(r$, rs or rsr"). QRS complex whth a higher voltage were depicted according to the ratio between the positive $(R, r)$ and the negatiwe waves $(q, Q, S$ and $\$$ ). For example a RS complex is defined by the presence of a QRS complex showing an intially positive deflection followed by a negative deflection of an even magnitude. Likewise, an Rs pattern means a QRS complex (> 0,3 mv) with an initial positive deflection followed by a smaller negative one. A septal $\mathbf{q}$ wave was defined as a $\mathbf{q}$ wave in surface leads $I$, aVL, and V6, with an amplitude $<25 \%$ of the $R$ wave and a width $<0.04$ second.

\section{Statistical Analysis}

Values are given as mean \pm standard deviation. The significance of differences $(p<$ 0.05 ) between groups of clinical, electrocardiographic or electrophysiologic parameters was assessed by Student"s / test or Fisher's exact test.

\section{RESULTS}

\section{Pre-ablation 12-lead ECG findings}

Minimal preexcitation, defined as subtle abnormalities suggesting the presence of preexcitation, with a QRS complex width within the normal range $(<0.12 \mathrm{sec})$ but with a short HV interval ( $<35$ msec), was present during simus rlhythm in 24 patients $(72 \%)$ (figure 1). The PR interval was not significanily different when comparing patients with $(125 \pm 21 \mathrm{msec})$ and without $(132+9 \mathrm{msec})$ minimal pre-excitation $(\mathrm{p}=$ ns). We found 2 patterns of the QRS complex (figure 2) during sinus rhythm: the most common one being an $\mathrm{rS}$ configuration in lead III. This was found in 20 patients. The other pattern in lead 1 II, an "rsR" was found in 2 patients. in the presence of an IS pattern in lead III, no q wave was found in lead 1 in 15 patients, (and in 8 patients in lead V6). Minimal preexcitation with absence of a $q$ wave in lead II (without $\mathbf{r S}$ in lead III), was seen in only 2 patients (patients 8 and 16, Table II). In three patients minimal preexcitation was not always manifest, as documented by 12 -lead ECG's taken on different days. Variability of minimal preexcitation in the same ECG was
seen in 2 patients (figure 3 ). 
$\frac{0}{0}$

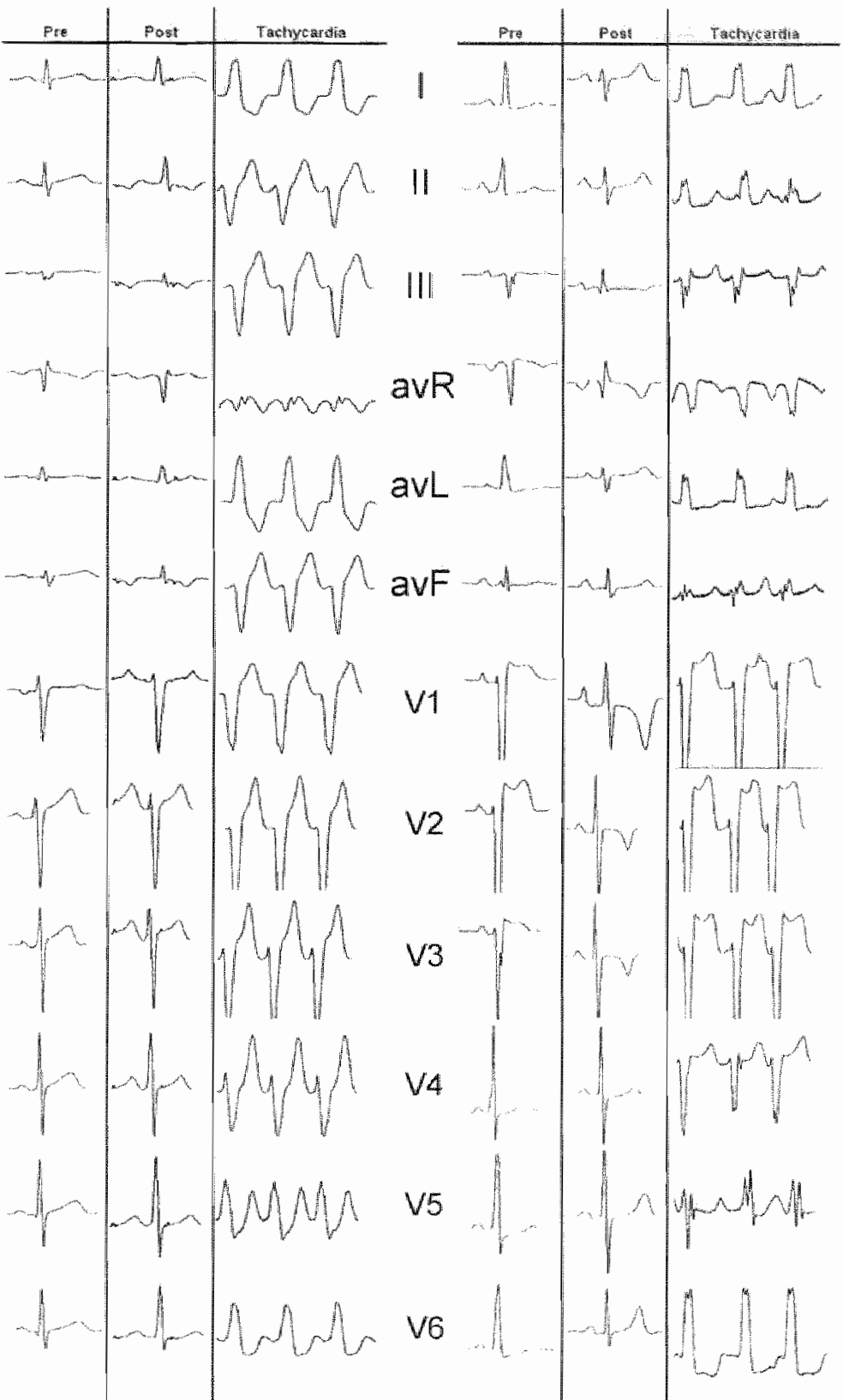

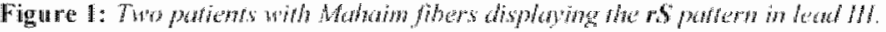

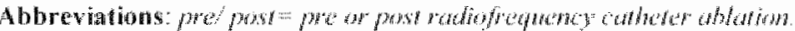




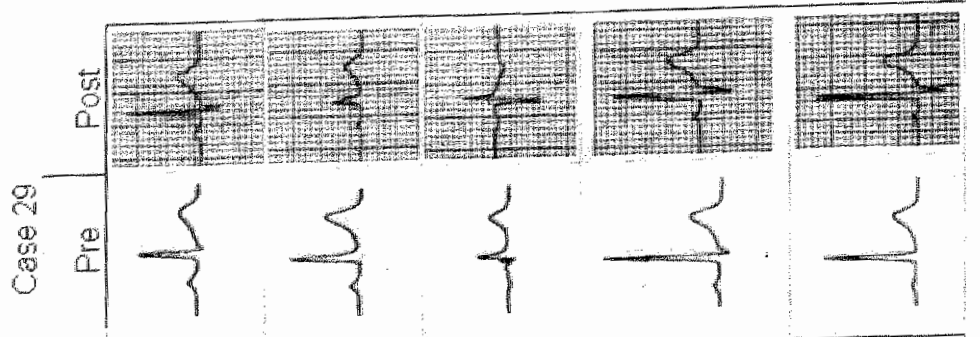

$\frac{5}{5}$
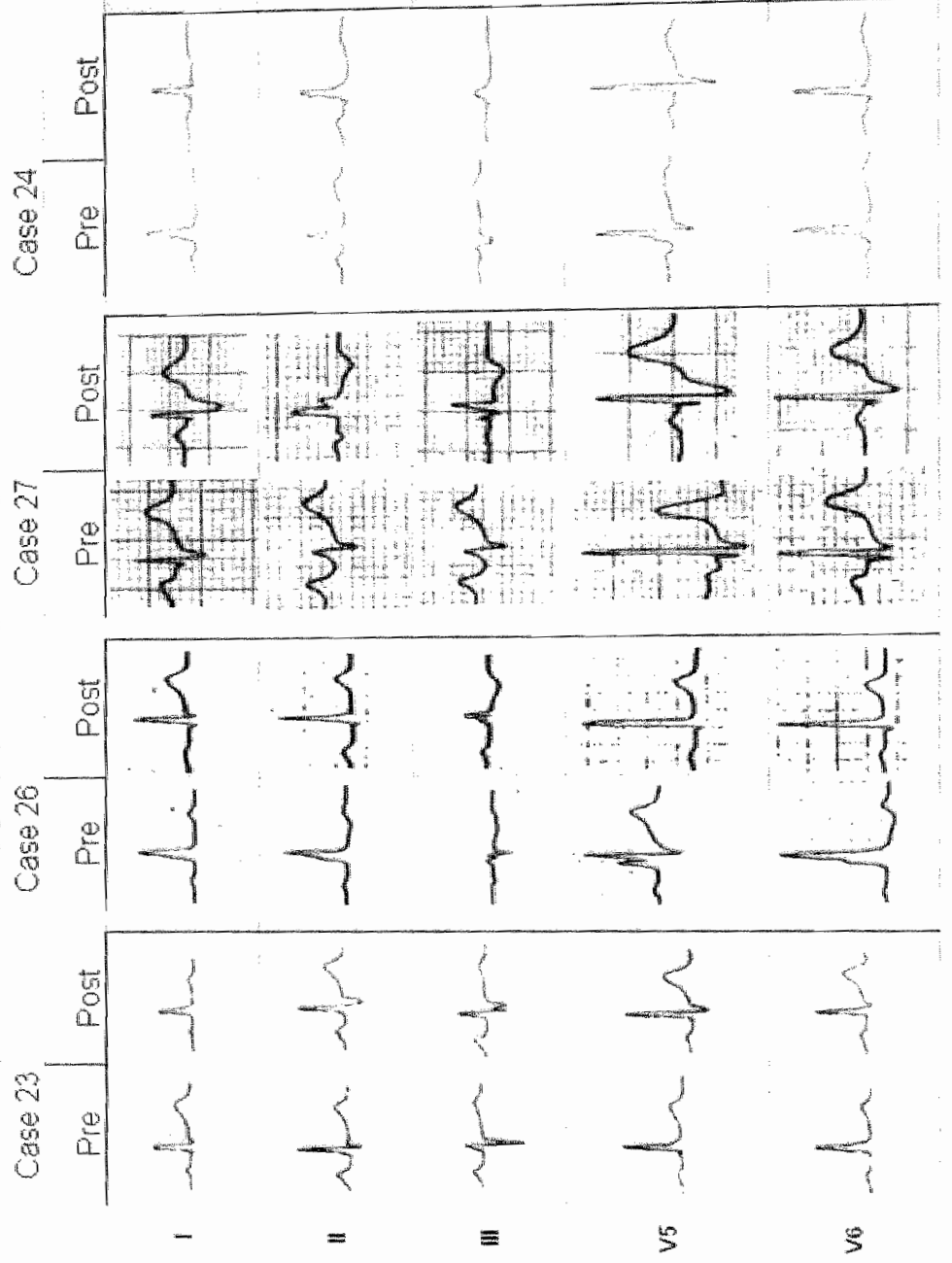

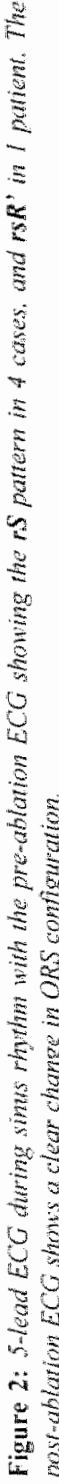


TABLE I

Clinical data

\begin{tabular}{|c|c|c|c|c|c|c|c|}
\hline n & $\operatorname{sex}$ & Aye & Site-TA & Arrllyythmia & Elystein & $\mathrm{CBT}$ & $\begin{array}{c}\text { Therapy } \\
\text { SRFpRF-dis }\end{array}$ \\
\hline 1 & $F$ & 31 & $\mathbb{L}$ & Antidromic AVRT Mahaim & & & $\$$ \\
\hline 2 & $\sqrt{F}$ & 32 & $\mathbb{L}$ & $\begin{array}{l}\text { Antidromic AVRT Malsain } \\
\text { AVNRT }\end{array}$ & & & RF \\
\hline 3 & $F$ & 19 & $P L$ & Antidromnic A WRT Malaim & Yes & & RF \\
\hline$\sharp$ & $F$ & 27 & $P L$ & $\begin{array}{l}\text { Antidromic AVRT Mallaim } \\
\text { Ortodrombic AVRT }\end{array}$ & Yes & RPS & $\begin{array}{l}\mathrm{RF}_{\mathrm{p}} \\
\mathrm{RFp}\end{array}$ \\
\hline 5 & $M$ & 52 & $P L$ & Preexcited atral fibrillation & & & RFp \\
\hline 6 & $M$ & 21 & $\mathbb{L}$ & Antidromtc AVRT Mahaint & & & $R F_{p}$ \\
\hline 7 & $\mathbb{F}$ & 23 & L & Antidomic AVRT Mahaim & & & $\mathrm{RFp}$ \\
\hline 8 & $F$ & 10 & A & Antidrontic AVRTMahatm & & & $\mathrm{RFp}$ \\
\hline 9 & $F$ & 23 & $\mathbb{L}$ & Antidromic AURT Mahaim & & & $\mathrm{RFp}$ \\
\hline 10 & $F$ & 25 & PL & Antidronitic AWRT Mahaim & & & RFp \\
\hline 11 & $M$ & 35 & $L$ & Antidronnic A WRT Mahaim & & & RFP \\
\hline 12 & $F$ & 42 & $L$ & Antidrontric AVRT Mahaim & & & $\mathrm{RFp}$ \\
\hline 13 & $F$ & 23 & $L$ & Aurtidronric AVRT Mahaim & & & $\mathrm{RFp}$ \\
\hline 14 & $F$ & 8 & $A L$ & Mahaim automaticity & & & PFp \\
\hline 15 & $M$ & 30 & $P L$ & Antidronth AVRT Mhathain & & & $\mathrm{RF}_{\mathrm{B}}$ \\
\hline 16 & $F$ & 27 & $L$ & Antidronute AVRT Mahaim & & & RFp \\
\hline 17 & $F$ & 19 & $L$ & Antidromic AVRTMalaim & & & RFp \\
\hline 18 & $M$ & 12 & $M S$ & Mahain automaticity & & & $\mathrm{RF}_{p}$ \\
\hline 19 & $F$ & 39 & $L$ & Antialromic AVRTMahaim & & & RFp \\
\hline 20 & $F$ & 15 & $A L$ & AVNRT + Mahatirn bystandef & & & $\mathrm{REF}$ \\
\hline 21 & $M$ & 13 & $L$ & Antidromic AURT/Maluaim & & & $\mathrm{RF}_{\mathrm{p}}$ \\
\hline 22 & $M$ & 15 & $A$ & Antidromic A URTMahaim & & & $\mathrm{RF}_{p}$ \\
\hline 23 & $F$ & 25 & $L$ & Antidromic AVRTMatatim & & & $\mathrm{RFP}$ \\
\hline 24 & $M$ & 18 & $L$ & Antidromic AVRT/Mahaim & & & $\mathrm{RF}_{\mathrm{p}}$ \\
\hline 25 & $F$ & 45 & $L$ & Antidronic AURTManaim & & & RFP \\
\hline 26 & $M$ & 24 & $L$ & Antidromic AURTMahaim & & & RF allis \\
\hline 27 & $M$ & 11 & $L$ & Antidromic AVRT Mahaim & Yes & & $\$$ \\
\hline 28 & $M$ & 20 & $L$ & Antidromic AURTMaliaim & Yes & $L L$ & RF-alls \\
\hline 29 & $M$ & 25 & $A$ & Antidromic AVRT Mahalin & & & RF alis \\
\hline 30 & $F$ & 20 & $P$ & Antidromic AVRT Mahaim & & & RF dis \\
\hline 31 & $F$ & 31 & $L$ & Antidromic AVRTMathain & & & RF-dis \\
\hline 32 & $F$ & 22 & $L$ & Antidrouric AVRT Mahain & & & RFp \\
\hline 39 & $M$ & 77 & $P$ & Antidromic AVRT Malnain & & & $\mathrm{PF}$ \\
\hline
\end{tabular}

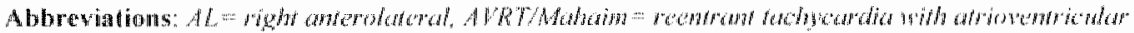

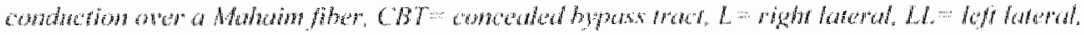

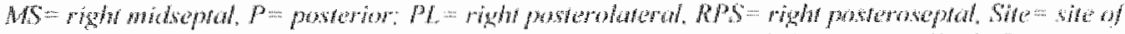

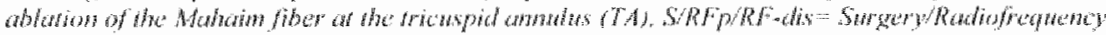

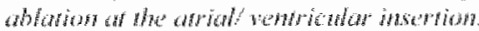



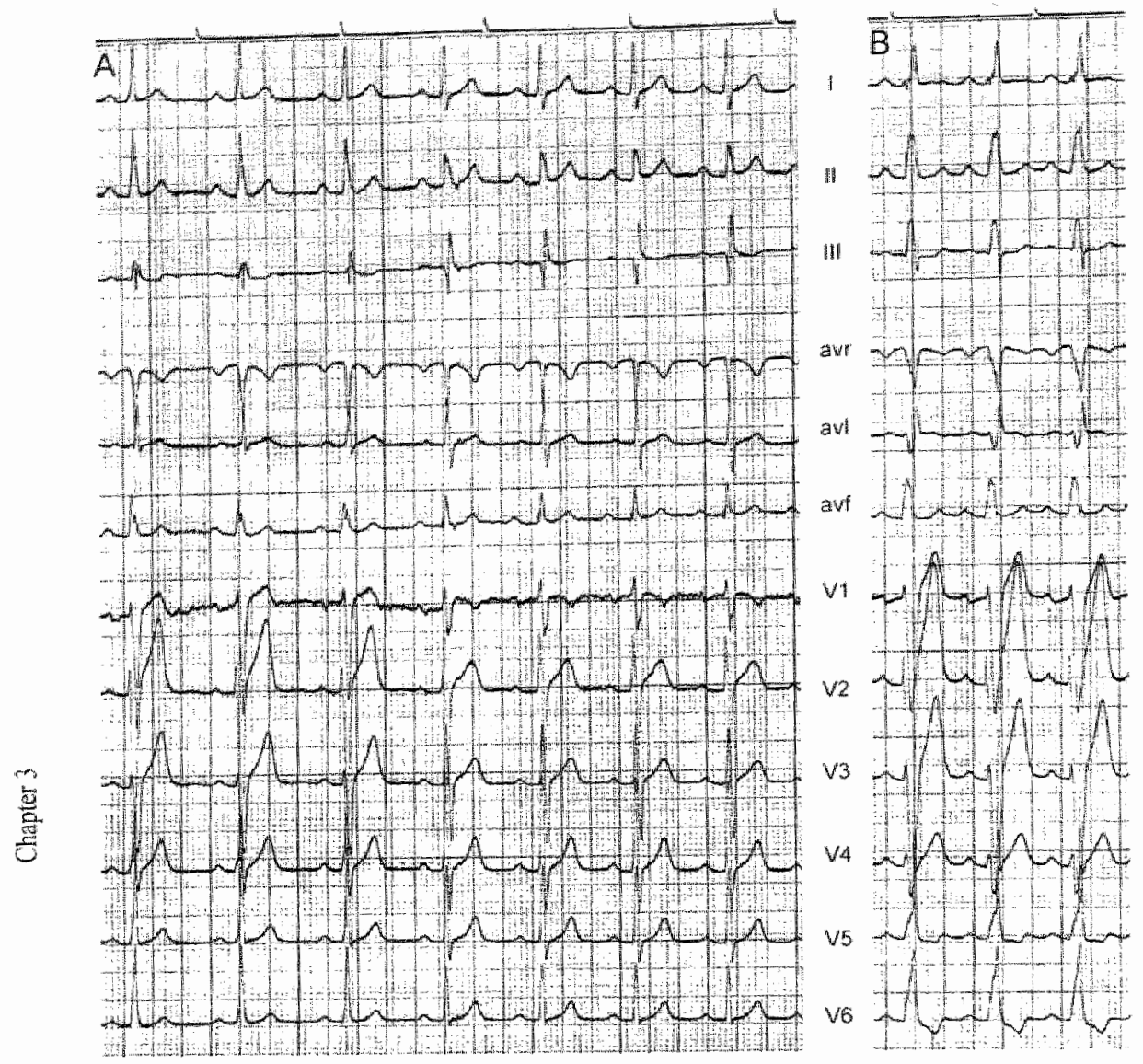

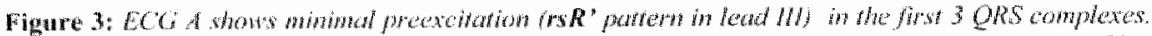

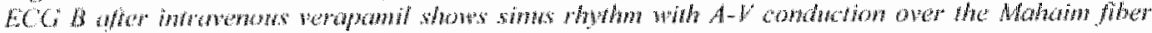

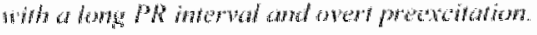

Intracavitary signals, Mahaim potential recording and right bundle and $H$ is bundle electrogana: We found that the AM anterval (atriumproxinal Mahaim potential interval) was always $20-40 \mathrm{msec}$ shorter than the $\mathrm{AH}$ interval in patients with minimal preexcitation. In patients without preexcitation the $\mathrm{AH}$ interval was shorter than the AM interval (figure 4).

ECG during tachycardia: We analyzed 29 patients with a circus movement tachycardia with anterograde conduction over the Mahaim fiber, one AVNRT with bystander Mahaim conduction, one with atrial fibrillation with anterograde conduction over the Mahaim liber and two automatic tachycardias arising in the 
TABLE II

Electrocandiographic data of Mahain fibers

BEFORE MAHAUMADLTION

\begin{tabular}{|c|c|c|c|c|c|c|c|c|c|c|c|}
\hline \multirow[t]{2}{*}{ th } & \multicolumn{5}{|c|}{ Sintis thythm } & \multicolumn{3}{|c|}{ Tachyoandia } & \multicolumn{3}{|c|}{ Sontrs ullyothm } \\
\hline & $\mathrm{PRi}$ & $\begin{array}{l}\text { ORS } \\
\text { willth }\end{array}$ & $\begin{array}{c}\text { Ietand III } \\
\text { DDE }\end{array}$ & $\begin{array}{r}11 \\
10\end{array}$ & $\begin{array}{l}9.66 \\
918 \\
\end{array}$ & lead1111 & axis & $\begin{array}{l}\text { ORS } \\
\text { width }\end{array}$ & $\begin{array}{c}\text { logul III } \\
\text { post }\end{array}$ & $\begin{array}{c}11 \\
\text { post }\end{array}$ & $\begin{array}{l}q \times 6 \\
\text { post }\end{array}$ \\
\hline 1 & 0.13 & 0,11 & 15 & mo & no & 15 & 0 & 120 & h? & $n$ & yes \\
\hline 2 & 0.14 & 0,11 & 15 & no & no & 15 & -30 & 130 & 10 & no & mo \\
\hline$y^{2}$ & 0,12 & $0,0.8$ & 75 & no & no & 15 & -45 & 1,0 & in & mo & mo \\
\hline 4 & 0,74 & 0,03 & qRs & no & no & 15 & 45 & 130 & 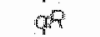 & no & no \\
\hline 5 & 0.92 & 0,09 & 15 & $n$ & $n a$ & QS & .45 & 180 & 15 & no & mo \\
\hline 6 & 0,72 & 0,00 & $p h$ & no & no & 15 & -30 & 120 & $g$ & no & no \\
\hline 7 & (1) & 0.08 & 15 & no & no & 85 & -15 & 120 & 9 & $n$ & yes \\
\hline 8 & 0.13 & 0,008 & $q \theta$ & yes & no & $R$ & 80 & 130 & $\phi$ & $m$ & yes \\
\hline 9 & 0,70 & 0,00 & 15 & yes & yes & 15 & 46 & 130 & ns & no & yes \\
\hline 10 & 0,12 & 0,1 & 15 & no & no & QS & 60 & 120 & $\sqrt{2}$ & yes & Y.es \\
\hline 11 & $(1, y)$ & 0.00 & 15 & yes & no & 15 & -15 & 120 & $\operatorname{ses}$ & yes & yes \\
\hline 12 & Q19 & 0, & 85 & $n_{0}$ & no & 5 & -30 & 130 & $g$ & $n$ & no \\
\hline 113 & $0,-13$ & 0,12 & 15 & no & yes & 15 & .30 & 120 & $g R$ & mo & yes \\
\hline 114 & 0,74 & 0.69 & 15 & no & no & $r s r^{\prime}$ & 0 & 120 & $g p$ & no & no \\
\hline 115 & 4.15 & 0,09 & 15 & yes & yes & $r S$ & -60 & 120 & ब9 & mo & no \\
\hline 1160 & 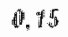 & 0,08 & $q p$ & no & mo & $r$ & -45 & 120 & $g$ & no & yes \\
\hline$\| 7$ & 0,4 & 0,08 & $q R$ & no & yes & 6 & .30 & 120 & $q p$ & 120 & yes \\
\hline 腰 & 0,10 & 0,11 & 15 & no & no & QS & -80 & 120 & $g h$ & no & yes \\
\hline $\mathbb{1 9}$ & 0,13 & 0,4 & $r S^{*}$ & yes & no & 19 & .45 & 120 & 8 & yos & no \\
\hline 20 & 11.12 & 0.7 & 15 & mo & no & 25 & -45 & 130 & $q Q$ & yes & yes \\
\hline 21 & 0,14 & 0,08 & $q \theta$ & no & yes & $r S$ & 0 & 130 & $q R$ & no & yes \\
\hline 22 & 0,0 & 0,00 & $q R$ & yes & yes: & $R$ & 80 & 130 & $q n$ & yes & yes \\
\hline 23 & 0,12 & 0.1 & 75 & no & no & $r s$ & -30 & 120 & $\phi$ & no & yes \\
\hline 24 & 0,11 & 0.1 & 15 & $n_{0}$ & no & 15 & -30 & 130 & $q R$ & yea & ties \\
\hline 25 & $(1,20$ & 0,00 & 5 & no & no & QS & .30 & 120 & $q Q$ & no & no \\
\hline 20 & 0.70 & 0.7 & is & $n o$ & $n$ & 13 & 0 & 120 & $q R$ & yos & yes \\
\hline $27^{5}$ & 0,12 & 0,1 & $r s$ & yes & yes & 8 & -30 & 100 & $r S R^{\prime \prime}$ & thess & yes \\
\hline $20^{\circ}$ & 0.12 & 0.7 & 15 & yes & yens & 15 & -75 & 120 & $R S$ & Hos & yos \\
\hline 29 & 0,12 & 0,09 & $\sin R^{\prime \prime}$ & no & no & 25 & 15 & 140 & $Q P$ & $\mathrm{no}$ & $n$ \\
\hline 30 & 0,14 & 008 & $q R$ & no & no & 05 & .60 & 120 & 9 & 170 & 170 \\
\hline 31 & 0,12 & 0,09 & $g \mathrm{p}$ & no & no & $B$ & .60 & 120 & $\mathrm{gr}$ & $n$ & mo \\
\hline 32 & 0.14 & 0,08 & $g R$ & yes & yes & 19 & .30 & 140 & $\mathrm{pP}^{\mathrm{i}}$ & yos & yes \\
\hline 33 & 0,14 & 0,09 & $S R^{\prime}$ & $n 0$ & no & 15 & -30 & 140 & $g h$ & $n 0$ & 100 \\
\hline
\end{tabular}

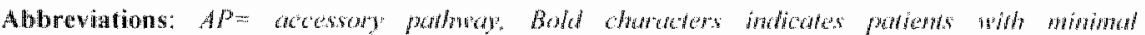

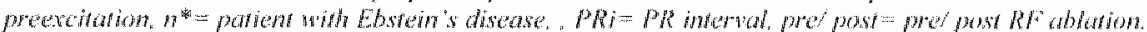

Mahaim fiber. During circus movement tachycardia the cycle length ranged from 430 $10250 \mathrm{msec}$. QRS width during tachycardia (Table II) waried from $120-140 \mathrm{mscc}$. All patients had a monophasic $R$ wave in lead $I$ and 30 out of 33 patients had $\mathrm{S}$ in VI during tachycardia ( 3 pts had a QS in V1). 
no preexcitation
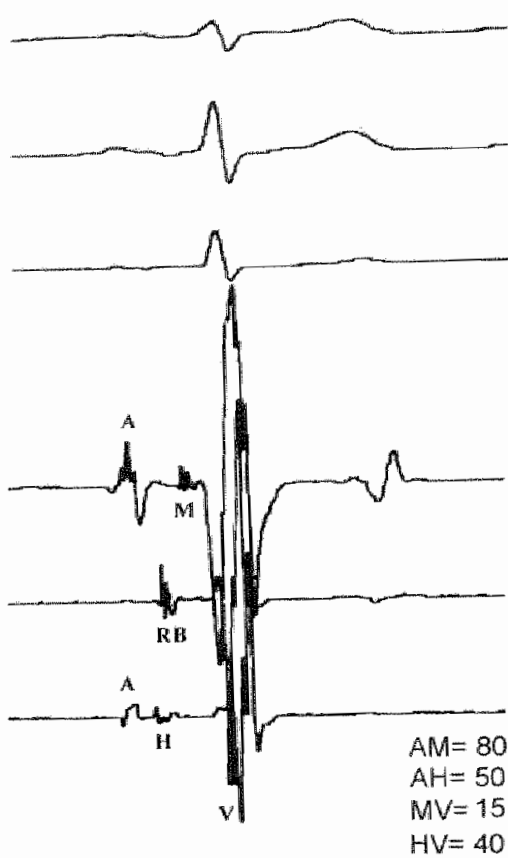

minimal preexcitation

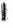

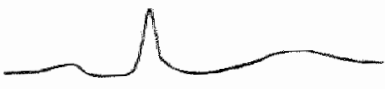

桄

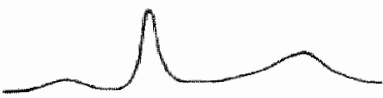

III

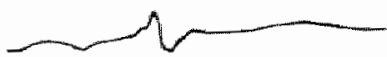

TIA

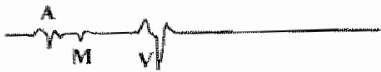

FB

HS

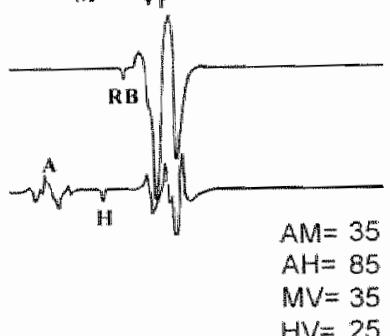

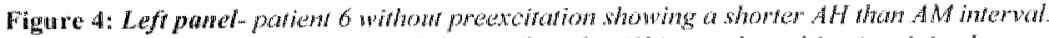

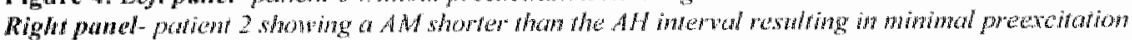

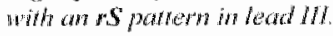

Comparison between the $\mathrm{CG}$ during simus rhythm and during tachycardia with anterograde conduction over the Mahain fiber

In all 20 pattents with an $1 S$ pattern in lead III we found a negative ORS complex in the same lead (either an $\mathrm{rS}$ or QS pattern) during tachycardia. Also the patient with atrial fibrillation showed a negative QRS complex. There were 9 patients without the is pattern in lead III during sinus rhyth, which showed left axis deviation during tachycardia. Three patients showed concordance between absence of an $\mathrm{rS}$ pattern during sinus rhythm and their tachycardia QRS patterm (all three patients had an anterior Mahaim) (figure 5).

\section{Post-ablation 12-lead ECG}

In the 24 patients showing minimal preexcitation in the $12-1$ ead ECG, 6 pattems were observed in lead III during sinus rhythm after Mahaim ablation. The most common QRS configuration was a $\mathbf{R}$ or $Q R$ pattern, found in 18 


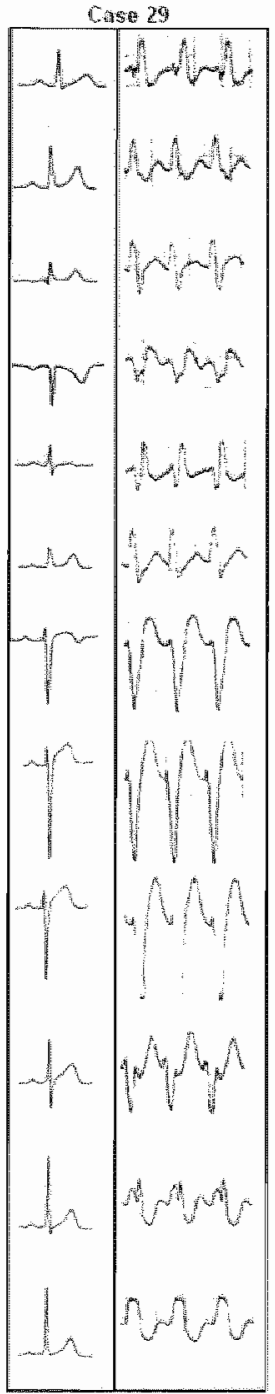

Figure 5: This pahent had an anferion

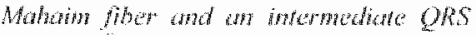

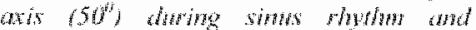

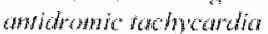

patients, Rs in I patient, RS in I patient, $r$ in 2 patients, $r R^{*}$ in $I$ patient and a rsr' in 1 patient. Assessment of the left precordial leads after ablation showed that in only 9 patients the previous $\mathbb{E C G}$ pattern changed with the development of a small q wave,

while the other patients showed the same pre-ablation QRS complex. Figure 2 gives examples when comparing the QRS pre and post ablation.

\section{Correlation between ECG findings and Mahaim fiber location:}

The rS morphology in lead III was not seen in the 3 cases with an anteriorly located Mahaim, or the 2 patients with a posterior Mahaim. The distribution of the atrial end of the Mahaim fiber in the 20 patients with an $\mathrm{SS}$ pattern in llead III during sinus rhythm along the tricuspid annulus is depicted in figure 6 . It is of interest that the atrial end of the Mahain fiber with an rS pattern in lead IIIl can be found over a large area around the tricuspid anmulus, from the anterolateral to the posterolateral and midseptal region.

The presence of an $\mathrm{CS}$ pattern in lead III during sinus rhythm in 200 matched controls:

We did a survey in 200 young individuals referred because of palpitations. Twelwe out of $200(6$ $\%$ ) matched controls (56\% females and mean age $23 \pm 12$ years) 


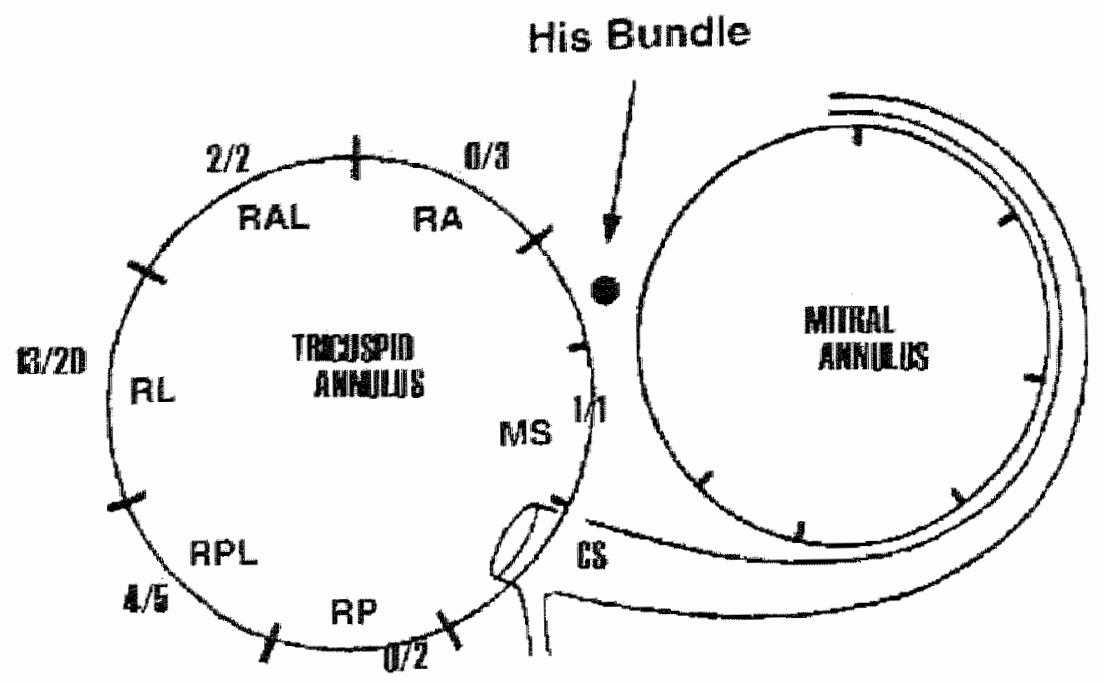

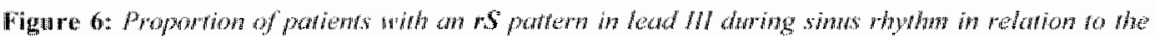

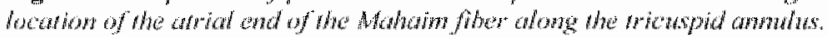

without heart disease and without a history of palpitations showed an rS pattern in lead III during sinus rhythm (Table III). However, all of them had a q wave in lead 1 ( $\mathrm{q} R$ or $q \mathbb{R} s$ patterns).

\section{TABLEIII}

Lead III pattern in 200 youmg individuals with palpitarions

\begin{tabular}{|c|c|c|c|c|c|c|c|}
\hline$q / \mathbb{R}$ & Rs:RS & $\mathrm{q} \mathrm{R}_{\mathrm{P}}$ & Rili & is & 19 & "si" & $\mathrm{ISR}^{\prime}$ \\
\hline $30 \%$ & $22 \%$ & $12 \%$ & $18 \%$ & $4 \%$ & $6 \%$ & $2 \%$ & $5 \%$ \\
\hline
\end{tabular}

\section{DISCUSSION}

When accessory atrioventricular pathways have conduction times approaching that of the nomal A-V conduction system, hitte or no preexcitation may be present during sinus rhythm. The reported incidence of minimal preexcitation in the 12 -lead $\mathbb{E C G}$ during sinus thythm in patients with decrementally conducting accessory pathways is low. Bardy at all and Klem et a ${ }^{16}$ did not find it in any of their patients. McClelland et al reported that only 1 of his 26 patients displayed preexcitation on the 12-lead 
ECO. When we realized the prevalence of the rS pattem in lead III in our patients wo examined previous reports dealing with decrementally conducting bypass tracts. We did find the rS pattem in lead III in many ECG's considered as normat in cardiology joumals $^{17.14-20}$ and textbooks ${ }^{21-2 ?}$. This suggests that the reported low figures of abnomal ECG's in patients with Maham bypass tracts is an underestimation. Some authors did acknowledge the presence of minimal preexcitation in 25 to $50 \%$ of the patients $^{14 .}$. We found an incidence of $72 \%$ of minimal preexcitation, mainly in the presence of an $\mathbf{r}$ pattem in lead III $(60 \%)$. It should be stressed that in these patients there is no classic delta wave. It is of interest that the $\mathrm{r}$ pattern was found in patients with decremental accessory pathways having their atrial end over a very large area around the tricuspicl annulus, from anterolateral to posterolateral, also in the only patient with a midseptal location (figure 6). This supports a ventricular insertion in a small anterolateral area in the right ventricle in or close to the exit of the right bundle branch and also explains (when ventricular activation starts at this site) the absence of a $q$ wave in lead $I$.

To validate the $\mathrm{rS}$ and $\mathbf{r} \mathbf{R}$ ' as abnomal patterns in lead III due to preexcitation of a small region of the right ventricle it was critical to show a positive relationship between those patterns in lead III during sinus rhythm and left axis deviation during tachycardia with anterograde conduction over the Mahaim fiber (ligure 1). All 20 patients with an $\mathrm{r}$ in lead III had left axis deviation $\left(\leq 0^{\circ}\right)$ during tachycardia. Another important step in validation is to show a clear change in QRS complex configuration after ablation of the decremental accessory pathway. Figure 2 depicts most of the patterns of QRS that emerged after successful ablation of the Mahaim fiber.

The fact that 9 patients did not show an $\mathrm{SS}$ pattern in lead III during sinus rhythm but an LBBB-like QRS with left axis deviation during tachycardia can be explained by impulse conduction over the Mahaim fiber during sinus rhythm slower than impulse conduction over the normal $\mathrm{A}-\mathrm{V}$ conduction system. We like other authors ${ }^{23}$ found day-to-day variability in the expression of minumal preexcitation. This is different from "intemittent" preexcitation which may occur in rapidly conducting $A P$ 's with long anterograde refractory periods. Our patients with variable expression of prexcitation did not have long refractory periods of their accessory pathway. There is one case report of sudden death in a patient with similar findings ${ }^{24}$. Conduction over Mahaim fibers can be so slow to that no ventricular preexcitation occurs even during atrial pacing. Still, these so-called latent Malnaim's are capable of being involved in antidromic tachycardias ${ }^{25}$

\section{Are all Mahaim fibers inserting close to or in the right bundle branch?}

Some Mahaim fibers are probably not inserting in that region. Our 3 patients with anterior Mahaim did not show an $\mathrm{rS}$ in lead $\mathrm{III}$ nor left axis devation cluring tachycardia, suggesting that in those fibers the ventricular insertion is not in the vicinity of the right bundle branch (figure 5 ).

\section{Septal $q$ wares}

In our population lead I was more sensitive for minimal preexcitation than lead V6. In patients with atriofascicular pathways inserting close to the apex, ventricular 
activation proceeds from an apical toward a basal direction, which may result in $q$ wave in lead V6. Minimal preexcitation due to leftesided accessory pathways can be better appreciated in lead $V 6$ which had been shown to be more sensitive than leads I and $\mathrm{arL}^{26}$.

\section{" $\mathrm{s} S$ " as a normall pattern in lead III}

It has been shown that an $\mathrm{rS}$ pattern in lead III can be found in normal individuals. This may occur during posterior displacement of the apex leading to $S$ waves in leads I. II and III $\left(S / S 2 S 3\right.$ pattern $\left.^{28}\right)$ and in counterclockwise rotation of the heart resulting in a $\mathbf{q} \mathbf{R}$ in lead $\mathbb{I}$ and $\mathbf{r S}$ in III. However, in those situations a nomal $\mathbf{q}$ wave in lead 1 is likely to be present. In our survey on $200 \mathrm{ECG}$ 's from young individuals with palpitations we found the rS pattern in lead III in $6 \%$, but always associated with a $q$ wave in lead I. No individual showed an $\mathrm{rS}$ pattern in lead III combined with the absence of a $q$ wave in lead I, a pattern that seems specific for patients with a Mahaim fiber.

\section{Limitation of the study - specificity of the $r S$ pattern in the general population}

The finding of an 15 pattern in lead III in $60 \%$ of the patients with Mahaim fibers is significantly higher than its occurrence in young persons with palpitations ( $p<$ $0,0001)$. Mahaim fibers comprise approximately $3 \%$ of the overt accessory pathways $^{29}$. Based on the prevalence of accessory pathways in the general population $^{30}(0,2 \%)$, the prevalence of Mahaim fibers would be $0,5-1: 10,000$. The specificity of an $\mathrm{rS}$ pattern in lead III associated with the absence of a septal $q$ wave will be close to $90 \%$ (if we assume one false positive in 1.000 individuals), albeit the sensitivity decreases to $45 \%$.

\section{CONCLUSION}

In young patients suffering from tachycardias the finding of a narrow QRS with an $r S$ pattem in lead III during sinus rhythm should raise the suspicion of the presence of a Mahaim fiber, especially in those showing absence of $q$ wave in lead $I$.

\section{Roforences}

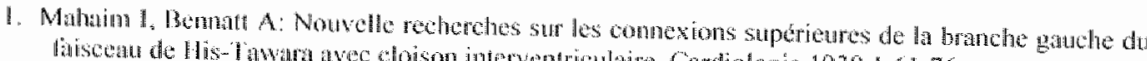

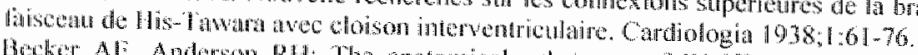

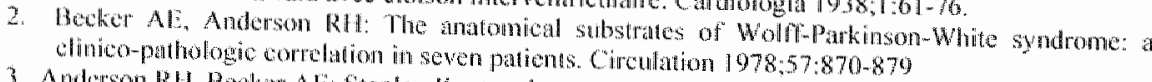

3. Anderson RU, Becker AE: Stantey Kent and accessory antiowentricular cannections. I Thoracic Cardowase Surg 1981:81:649-658.

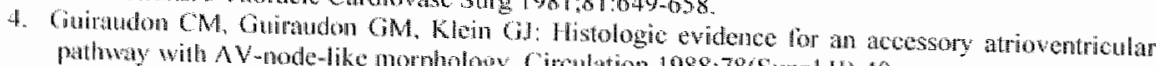

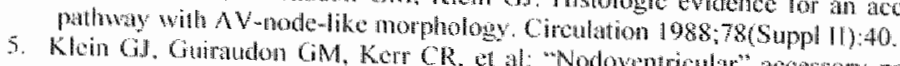

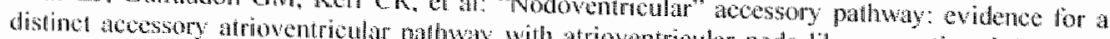
Candal 1988, 11:1035-1040.

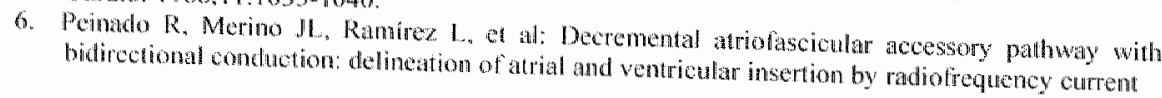


application. J Cardiovascular Electrophysio $2001: 12 ; 439492$.

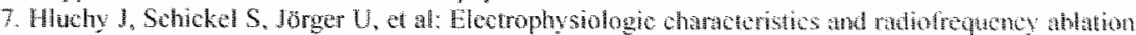

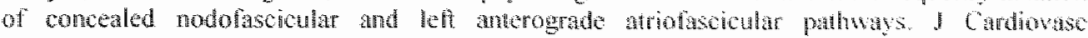
Electrophysio $2009 \% 11: 211-217$.

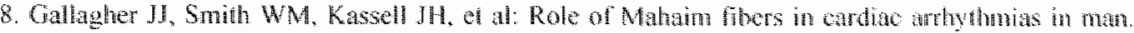
Circulation $1981,64: 176,89$.

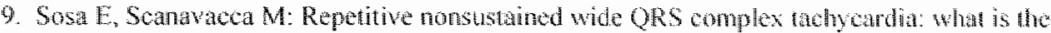
tachyouda mechanism? J Cardiovasc Electrophysiol 2001:12,977.978.

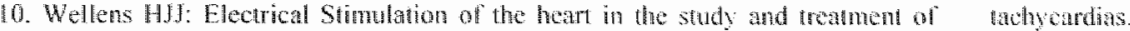
Universily Pank Press, Batimore, 1971.

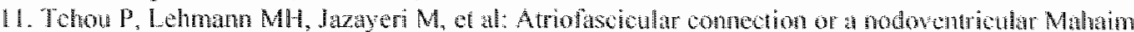

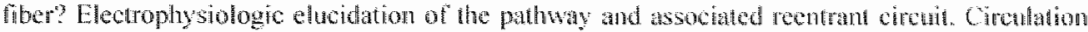
$1988,77837 \times 48$.

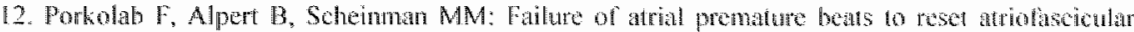
tachyeardia. Pacing Clin Electroplysiol 1999;22-528-530

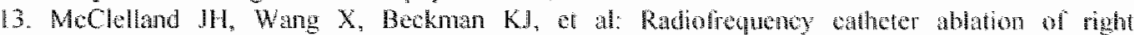

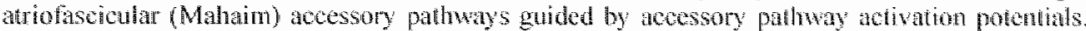
Circulation 1994:89:2655-66.

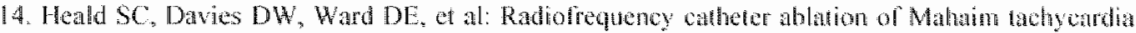

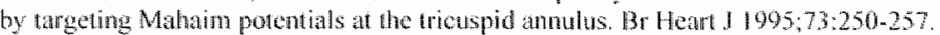

15. Stemich EB, Gerken I.M. Vrandecic MO. Apprassal of "Maham" automatie tachyoutha: I Caraiovase blectrophysio $2002 ; 1,3: 244-249$.

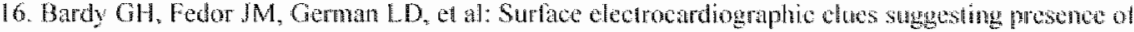
a nodolascicular Maham biber, J Am Coll Cardiol 1984:3:1 161-1168.

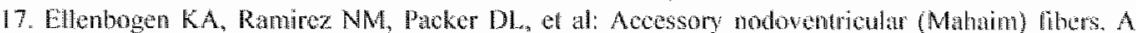
anical revew. Pacing Clin Clectophysiol 1986;9:868-884.

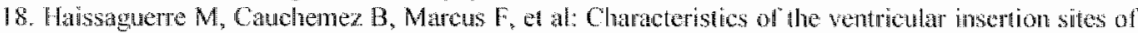
necessoly pathways with anterograde decremental conduction properices. Cureulation $1905,91: 1077-1085$.

19. Klen LS, Hacket K, Zipes DP. at al: Radiofrequency catheter ablaton of Mataim fibers a the tricuspid anmulus. Circulation $1993,87: 738-747$

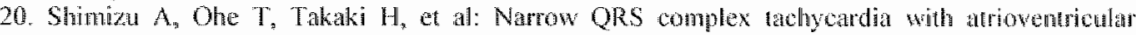
dissociation. Pacing Clin Electrophysiol 1988,11:384-393.

21. Mitteman RS, Hung SKS: Ablation of Mahom fibers. In: "Radofrequency catheter ablation ot

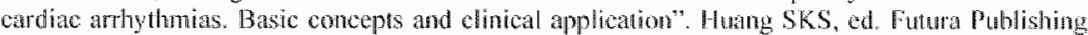
Co, Amonk, NY, USA, 1995, p 352.

22. Josephson ME Prexcitation syndromes. In: "Clinical Cardiac Blectrophysiology, "iechniques and Interpretations". Philldelphia, PA, USA Lippincon Williams Wilkins, 2002, pe 404.

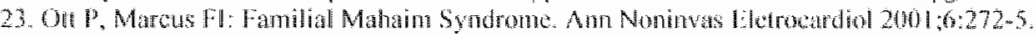

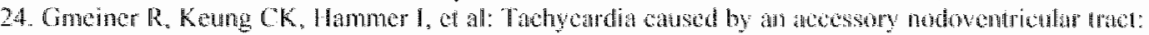
7 chicopalhologic correlation. Eur Hoat J 1964,$5 ; 23,9-242$.

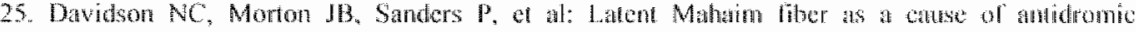

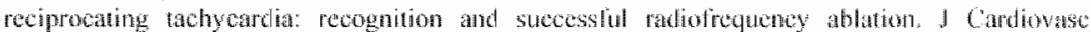
Cieconoplysiot $2002 ; 13: 74-78$

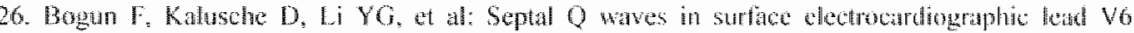

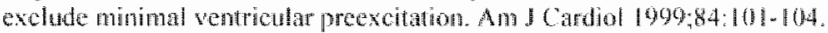

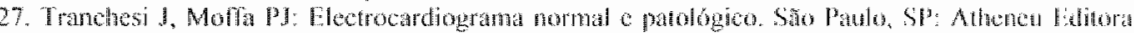
1.7DA. 1983, p. $86-9 \|$

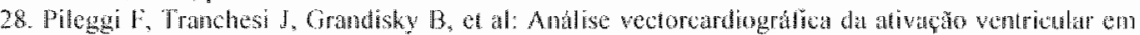

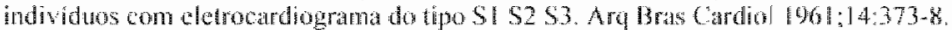

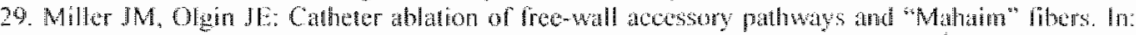

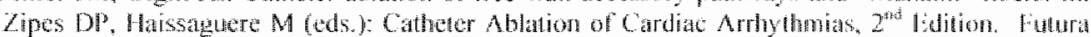
Publishing Co. Inc Ammonk, NY, 2002, pp277-303.

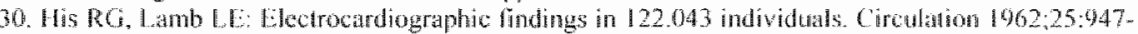
061 . 


\section{Chapter 4}

\section{Tachycardias associated with Mahaim fibers: a review}

Eduardo Back Sternick MD*

Hein JJ Wellens MD, PhDt.

Biocor Instituto*, Nova Lima, Brazil; Cardiovascular Research Institute $\ddagger$, Maastricht, The Netherlands. 


\title{
INTRODUCTION
}

Accessory pathways with decremental conduction are fascinating and uncommon. Their incidence is estimated to be around $3 \%$ of the population with accessory pathways'. Its rarity played a role in our slow progress in understanding their structure and properties ${ }^{24}$. It took some decades to discover that the proximal end arises in the right atrium and not the A-V node in the majority of Mahaim libers ${ }^{\text {s- }}$ ${ }^{6}$, with some rare reports of left-sided Mahaims ${ }^{2.3}$. We now know that nodoventricular and nodofascicular fibers are rare but they may play a role in tachyarrhythmias with successful ablation of such structures in the right midseptal region.12. Fasciculoventricular pathways do not have decremental conduction ${ }^{9-15}$ but because they originate in the normal sub $\mathrm{A}-\mathrm{V}$ nodal conduction system they are still included in the original family of Mahaim fibers (table I). In this review we will discuss the clinical spectrum of cardiac arrhythmias related to Mahaim fibers.

\section{ECG DURING SINUS RHYTHM}

Analysis of the 12-lead ECG during sinus rhythm can be helpful in the clinical diagnosis of a Malnaim fiber. According to our experience up to $70 \%$ of the patients manifest minimal preexcitation ${ }^{16}$, which can be overlooked because preexcitation is subtle and associated with a narrow QRS complex. There is some day-to-day variability in the occurrence of preexcitation and analysis of ECG's taken at different days can improve sensitivity. The most common abnomality is an $\mathrm{rS}$ pattern in lead III (figures: $8 \mathrm{~A}$-before MAT- and 10B), with a small $r$ amplitude and an $r / S$ ratio less than $1 / 3$. Absence of a septal $q$ wave in the left precordial leads and especially in lead I may occur in association with the rS patten ${ }^{16}$. The ECG during sinus rhythm in short atrioventricular Mahaim fibers is usually fully preexcited ${ }^{1.9}$.

A latent form of Mahaim fiber has been described, where preexcitation is only present during antidromic tachycardia and not during both sinus rhythm and atrial pacing. It is sometimes difficult to make the differential diagnosis with ventricular tachycardia. It is important in those cases to deliver late premature right atrial beats during tachycardia at a time of low septal right atrium and lis bundle refractoriness. If ventricular activation can be advanced a latent Mahaim fiber is likely to be engaged in the circuit ${ }^{18.19}$.

\section{CLASSIFICATION OF THE DIFFERENT CARDIAC ARRHYTHMIAS THAT MAY OCCUR IN THE PRESENCE OF MAHAIM FIBERS:}

\author{
Arrhythmias related to atrioventricular/ atriofascicular (AV/AF) fibers \\ 1. Antidromic tachycardia with anterograde conduction over a long Mahaim liber \\ 2. Antidromic tachycardia with anterograde conduction over a short Mahaim fiber \\ 3. AVNRT with bystander Mahaim conduction \\ 4. AVRT with bystander Mahaim conduction \\ 5. Spontaneous fast automatic tachycardia arising in the Mahaim fiber \\ 6. Spontaneous slow automatic rhythm arising in the Mahaim fiber
}


7. Automaticity induced by radiofrequency ablation at the site of atrial insertion of the Mahaim fiber

8. Atrial fibrillation with amerograde conduction over the Mahaim fiber

9. Non-reentrant preexcited tachycardia due to simultaneous dual Mahaim conduction

\section{Arrhythmias related to nodoventricular/ nodofascicular (NV/NF) fibers}

10. Antidromic nodoventricular/ nodofascicular tachycardia with $\mathrm{A}-\mathrm{V}$ dissociation

11. Orthodromic tachycardia via a "concealed" NF fiber

Fasciculoventricular (FV) fibers

12. Orthodromic tachycardia with a "bystander" fasciculoventricular pathway

13. Atrial fibrillation with anterograde conduction over a fasciculoventricular pathway

\section{ANTIDROMIC TACHYCARDIA WTTH ANTEROGRADE CONDUCTTION OVER A LONG MAHAIM FIBER}

This is the most common type of tachycardia associated with Mahaim fibers.
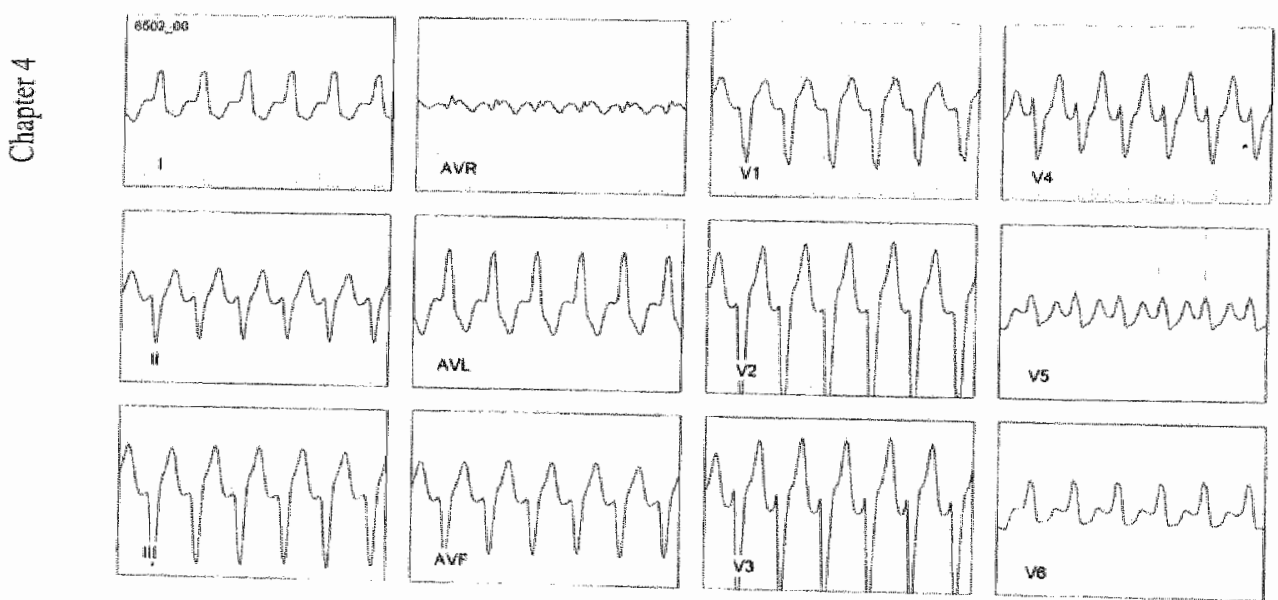

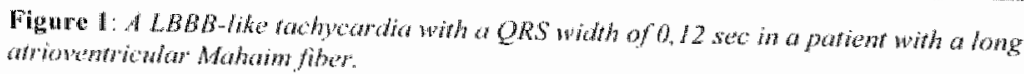

The tachycardia (Figure I) is usually regular with a mean cycle length of $309 \pm 52$ $(220$ to 450$)$ msec. The QRS complex width is usually around $0,13 \pm 0,01$ sec (range 0,11 to 0,15 ), shows a left bundle branch block like configuration with a smooth slope of the downstroke in $\mathrm{V} 1$, and a frontal plane axis between 0 and $-75^{\circ}$. In rare cases of an anteriorly located Mahaim fiber the axis can be around $+60^{\circ}$. The QRS width during tachycardia is related to the distal site of insertion of the Malnaim fiber. The closer to the right bundle branch the narrower the QRS. Retrograde VA conduction over the normal conduction system is associated with. 
a time interval between $100-140 \mathrm{msec}$. This will also depend whether $V-A$ conduction goes over the right or left bundle branch. The retrograde $P$ wave can usually not be recognized because it falls within the linal portion of the ORS complex. The major differential diagnosis is an SVT (orthodromic atrioventricular reentrant tachycardia using an $\mathrm{A}-\mathrm{V}$ bypass tract retrogradely or more rarely an AVNRI with LBBB).

\section{ANTIDROMIC TACHYCARDIA WITH ANTEROGRADE CONDUCTION OVER A SHORT MAHAIM FIBER}

Short atrioventricular Mahaim fibers usually have wider QRS complexes and also longer VH intervals. Figure 2 shows a LBBB-like tachycardia of $215 \mathrm{~b} / \mathrm{min}$, and a $-30^{\circ}$ QRS frontal plane axis in a patient with Ebstein's disease and an associated right

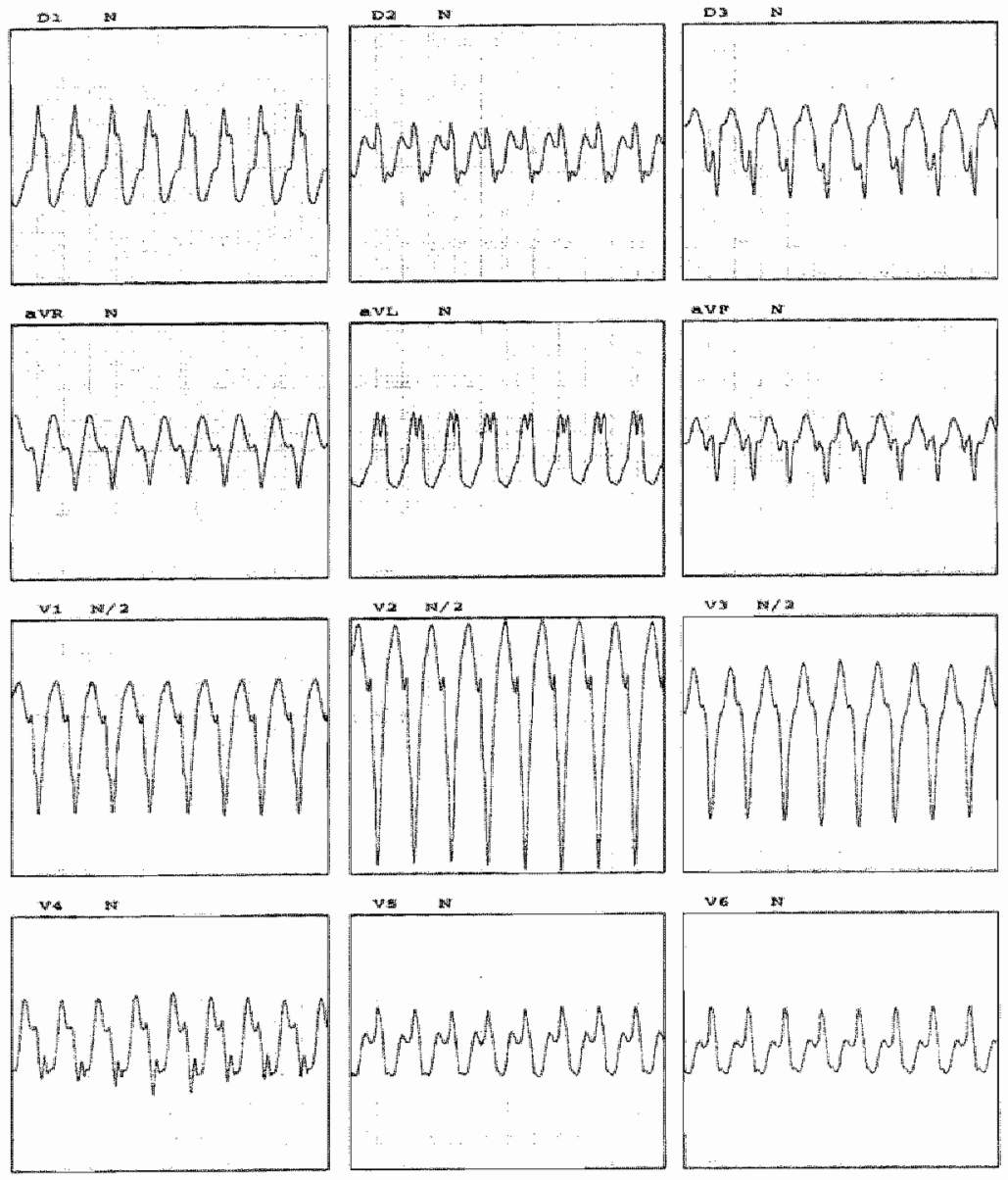

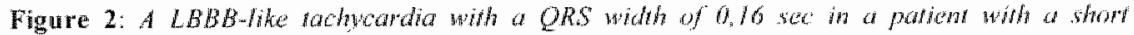
atriowentricular Maham fiber. Note the motch in the s wave in Vh. 
postero-septal accessory pathway, which is utilized as the retrograde limb of the tachycardia circut. The QRS complex is wide $(0,16$ sec), there is a notch at the downstroke of $\mathrm{V} \mathrm{I}$ indicating ventricular activation starting in the free wall of the right ventricle consistent with a short atrioventricular Mahaim fiber. Ventricular activation mapping showed early activation at the level of the tricuspid annulus. It is expected that the longer the distance berween the distal insertion of the Mahain fiber and the right bundle branch the wider the QRS will be. The V-A conduction interval was $80 \mathrm{msec}$, and the retrograde $\mathrm{P}$ wave could not be seen on the 12 lead ECG, being buried inside the QRS complex. Ebstein's disease is a common diagnosis in patients with

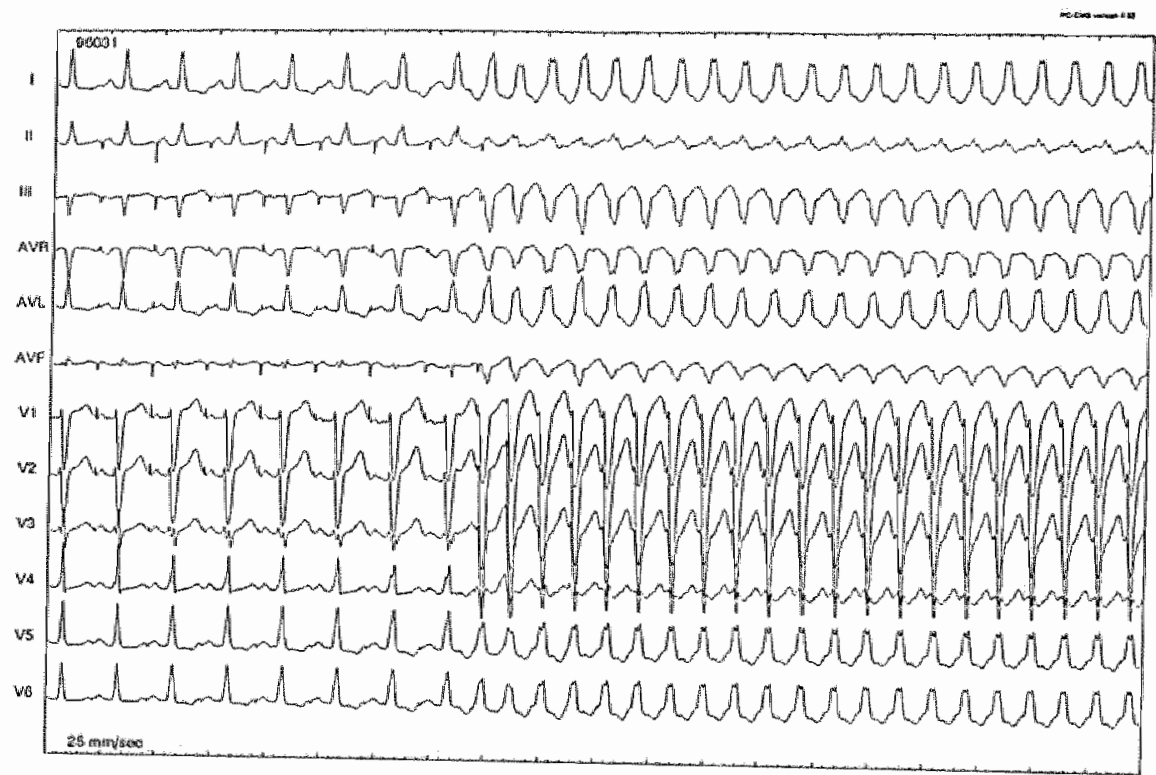

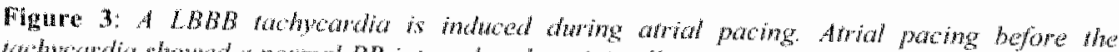

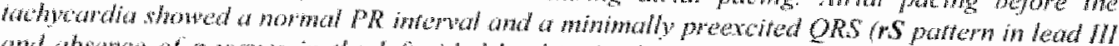

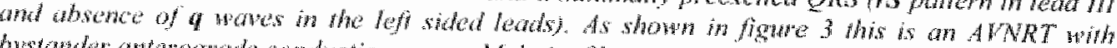

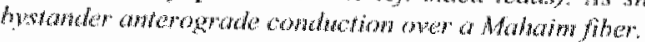

Mataim fibers with an incidence ranging from 10 to $50 \%(20 \%$ in our series of 40 patients). Mahaim fibers are associated with an overt or a concealed accessory A$\checkmark$ pathway in up to $20 \%$ of cases. The AP location is usually right-sided or postero-septal and only exceptionally left-sided ${ }^{21}$

Such $A P^{2}$ can be used in $V-A$ direction during antidromic tachycardia. 


\section{AVNRT WITH BYSTANDER MAHAMM CONDUCTION}

Atrioventricular nodal reentrant tachycardia (AVNT) can be associated with a Mahaim fiber ${ }^{10,22.23}$. It can occur as a narrow ORS Inchycardia, usually with a faster rate than the antidromic reciprocating tachycardia or it can be the presenting arthythmia showing ventricular preexcitation by bystander anterograde conduction over the Mahaim fiber (figures 3 and 4). The QRS complex would be indistinguishable from the real antidromic reciprocating tachycardia because anterograde $A-V$ conduction goes over the Mahaim fiber. The finding of fusion beats during tachycardia is the major clue to diagnose such a mechanism but fusion is almost never present during SVT, when it does, it is usually at induction. During the EP study block in the accessory pathway can be achieved by means of premature stimuli at the atrial (figure 4) or ventricular level. Sometimes no spontaneous or induced fusion beats can be seen and the diagnosis of bystander anterograde Mahaim conduction can only be made when an AVNRT with the same rate can be induced after ablation of the Mahaim fiber ${ }^{24}$.

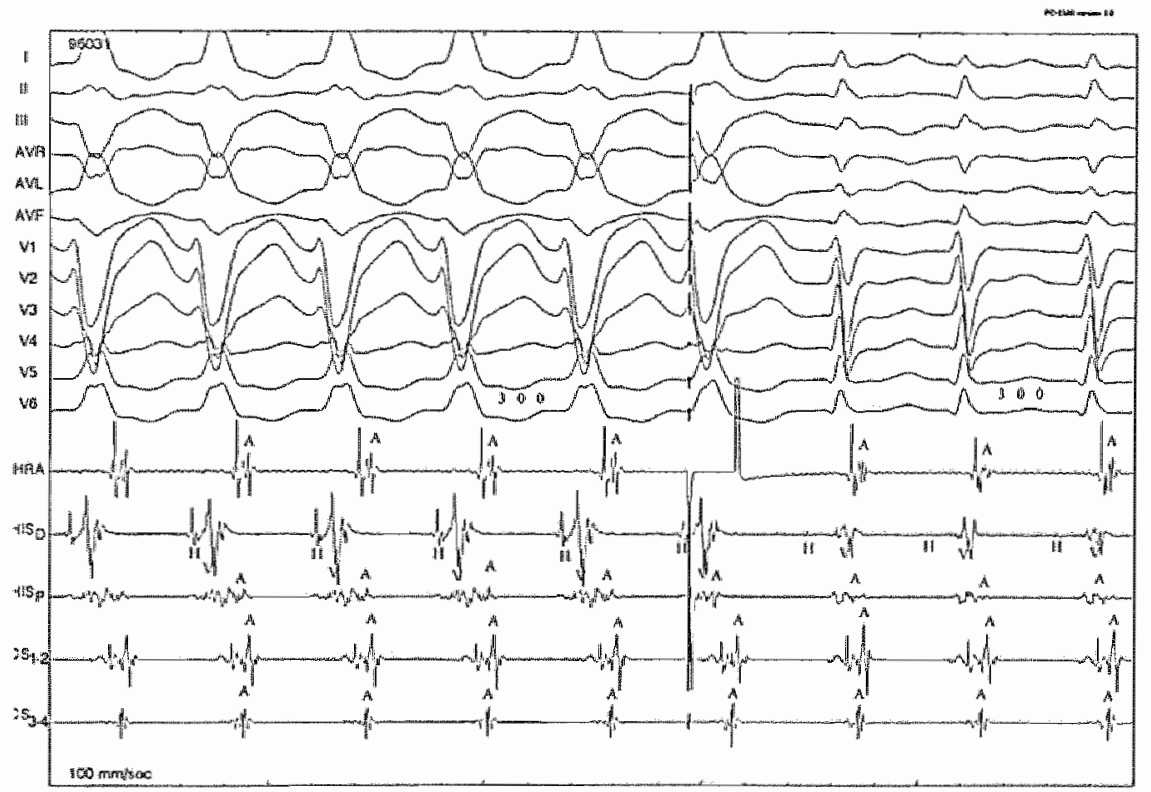

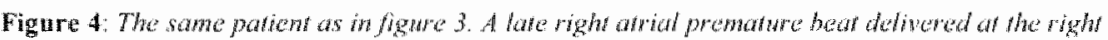

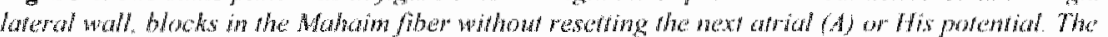

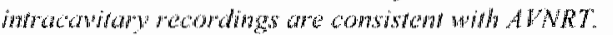

\section{AVRT WITH BYSTANDER MAHAIM CONDUCTION}

In our series of 40 patients with "Mahaim variants" bypass tracts, associated rapidly conducting accessory pathways occurred in $20 \%$ (5 WPW and 3 concealed APs). In spite of that common association, we induced an orthodromic 
reciprocating tachycardia with bystander anterograde Maham conduction only once (figure 5 ).

The usual scenario (in 3 patients with multiple pathways) was the induction of 2 different unrelated lachycardias, an orthodromic AVRT and an antidromic tachycardia using the Mahaim pathway as the anterograde limb and the A-V node rettogradely. However, complex reentrant circuits may be seen. The diagnosis of an orthodromic AVRT with bystander anterograde Mahaim conduction is most likely if retrograde conduction during a pre-excited tachycardia is excentric. Noninducibility after ablation of the rapidly conducting accessory pathway is the definite proof that the decremental pathway does not participate in the circuit ${ }^{24}$.

\section{SPONTANEOUS FAST AUTOMATIC TACHYCARDIA ARISING IN THE MAHAIM FIBER}

Spontaneous automaticity arising in the Mahaim fiber has been described occasionally ${ }^{25}$. The clinical presentation of such rhythms can be as premature beats in a bigeminal pattern, slow rhythms resembling accelerated idioventricular rhythm or as fast non-sustained bursts of repettive tachycardia. We have seen 2

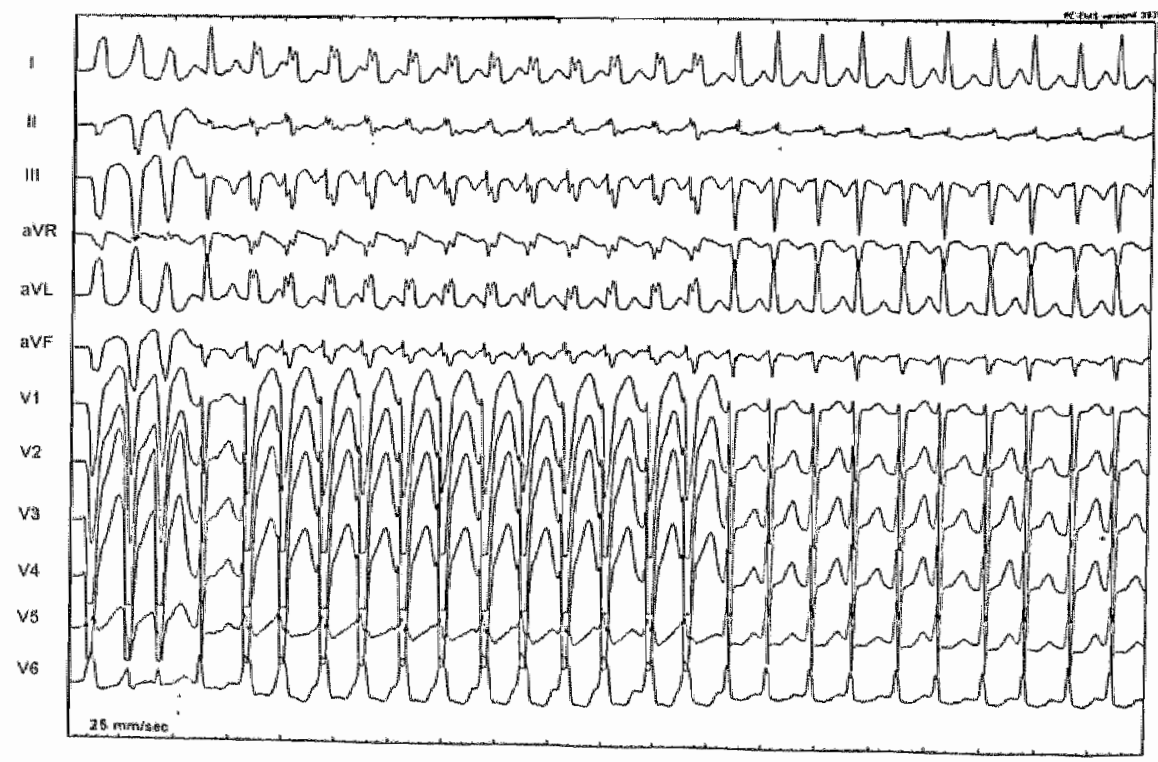

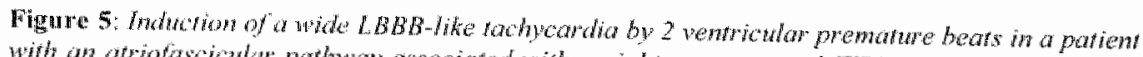

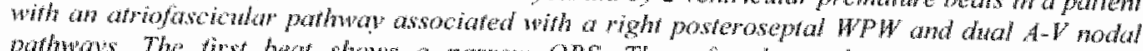
pathwass. The first beat shows a warow QRS. Therequen bystander anterosmade Maham

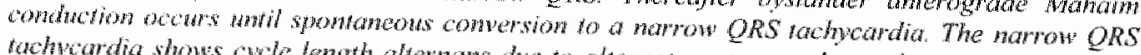

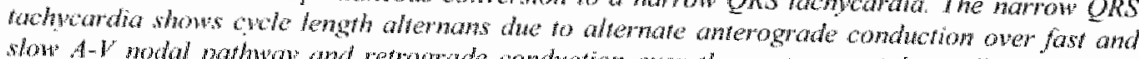

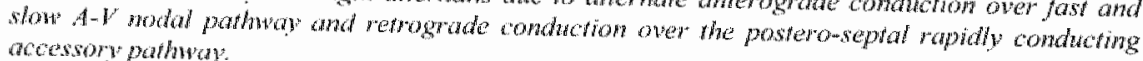


patients with frequent episodes of repetitive atumatic rhythms as the prasenting armythmia, without a true antidromic tachycardia. There was no V-A conduction over the A-V node and over the Mahaim fiber (figure 6). The QRS morphology during tachycardia equals the one during atrial pacing at the same rate. Patients having spontaneous
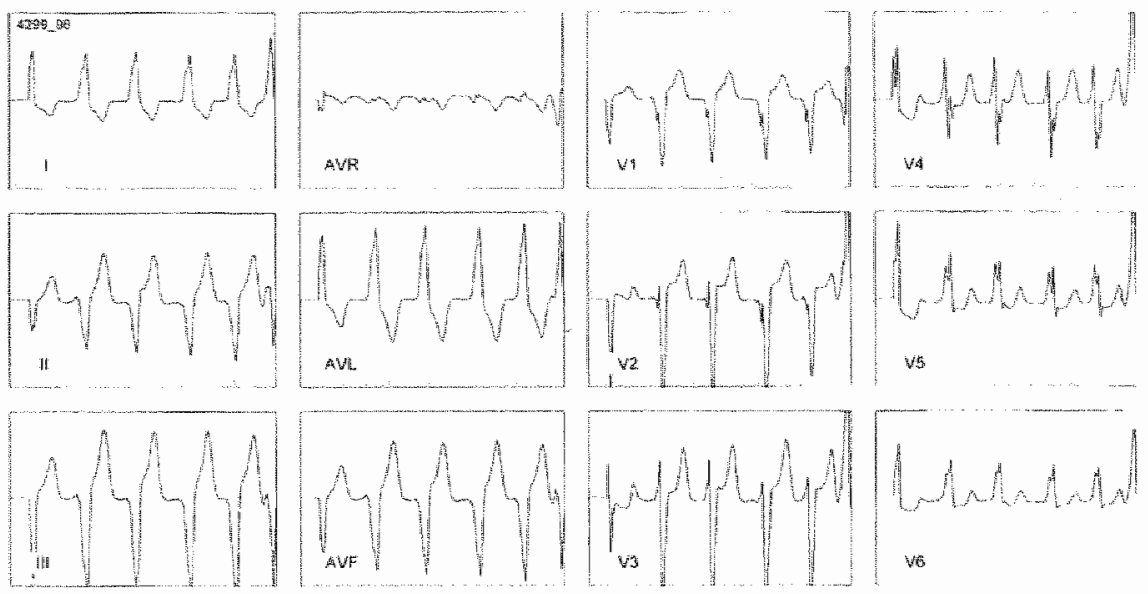

Figure 6: The 12Head ECG shows a slow LABB-like tachycardia (ORS= $0.12 \mathrm{sec}$ with $\mathrm{A}-\mathrm{V}$ disseciation itregular RR imervals and a cycle length between $400-480$ msec. The frowtal ORS axis is $-50^{\circ}$.

automaticity associated with Mahaim fibers are younger (15-7 years) then patients (26+13 years) with Mahaim fibers without spontaneous automaticity"

\section{SPONTANEOUS SLOW AUTOMATIC RHYTHM ARISING IN THE MAHAIM FIBER}

Spontaneous slow automaticity arising in Mahaim fibets is an infrequent rhythm that was present in only $7,5 \%$ (3 out of 40 ) of our patients. Those rhythms are clinically silent (figure 7) resembling an AIVR. In 2 of our 3 patients spontaneously occurring automatic beats triggered episodes of antidromic tachycardia (figure 8). Automaticity was abolished after successful catheter ablation of the Maham fiber. In both patients it was required to terminate all heat induced Mahaim automaticity (MAT) ${ }^{27}$, to abolisth conductionover the Mahaim fiber and spontaneous Mahaim automaticity. 


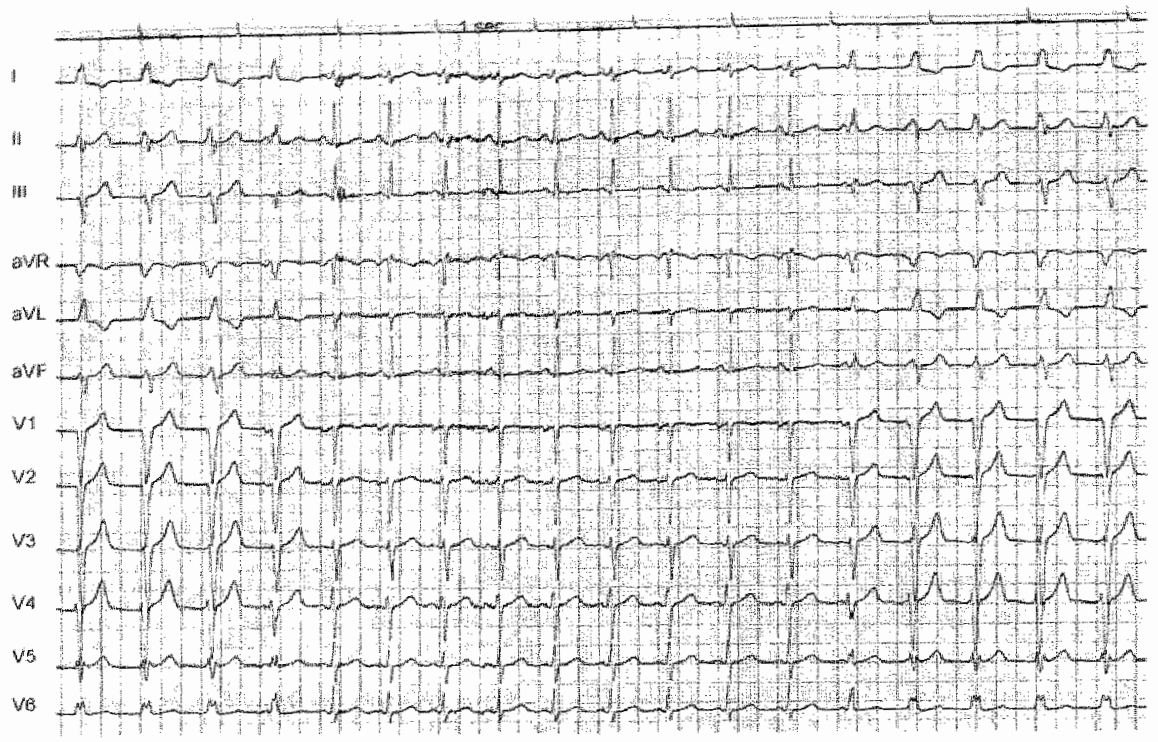

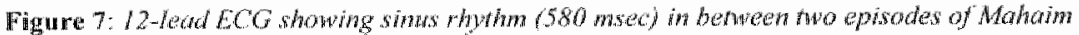

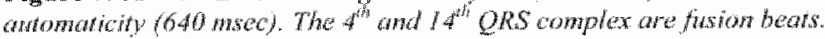

\section{AUTOMATICITY INDUCED DURING RADIOFREQUENCY ABLATION AT THE ATRIAL INSERTION OF THE MAHAIM FLBER}

Heat induced attomaticity is a long known phenomenon occurring when ablating the slow $A-V$ nodal pathway in patients with AVNRT. Some authors believe that heating thecompact node at a distance is the most likely explanation ${ }^{29}$. Others believe that it is caused by heating of $\mathrm{A}-\mathrm{V}$ nodal tissue at the posterior extensions of the A-V node. It is also known that regular atrial or ventricular myocardium does not generate such autonaticity. The observation of automaticity during ablation of Mahaim fibers (MAT) was reported in the early 90's. McClelland et al ${ }^{30}$ found it in 11 out of 23 patients, Heald at al' found it in 12 out of 16 patients and called it "stuttering block", Braun et al found it in 15 out of 15 patients. We found it in 30 out of 33 patients, dnuring ablation at the tricuspid annulus targeting the Mahaim "compact node". MAT usually starts immediately or a couple of seconds after current delivery. Automaticity may be short-lived, as short as 4 beats or very long-lasting up to 90 seconds (figure 9). The attitude toward the occurrence of MAT changed from previous concem about stability of the catheter $^{32}$ to a desirable event meaning a hallmark of successful ablation. We belicve that in some cases with prolonged automaticity, sometimes associated with spontaneous autonatic rhythms, complete termination of MAT may be required for long-term success ful outcome. 


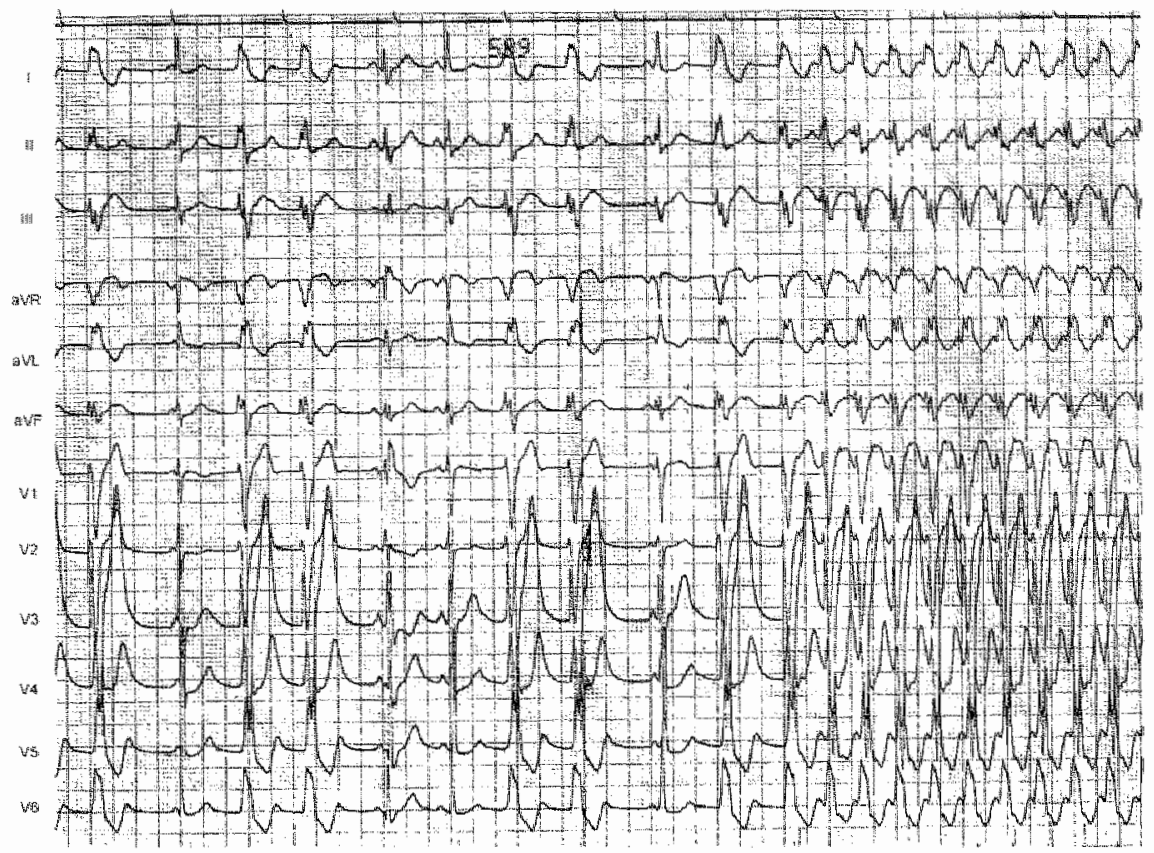

Figure 8: 12-1ead ECG showing antomaric bears anong minimally preexcited sinus beats.

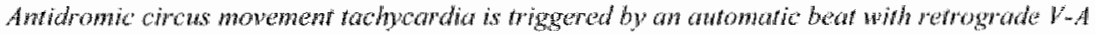
conduction whough the A-V wode.

\section{ATRIAL FIBRILLATION WITH ANTEROGRADE CONDUCTION OVER THE MAHAIM PATHWAY}

We saw spontaneous atrial fibrillation as the presenting arrhythmia in patients witl Mahain hibers only once in 40 patients. It was a 50 yoar odd patient (ligure 10) without retrograde conduction over the fiber. After successful ablation of the Mahaim fiber atrial fibrillation did not recur during a follow-up of 2,5 years. Miller et $a^{25}$ ablated a Mahaim fiber during atrial fibrillation. After ablation atrial fibrillation could not be re-induced.

We reviewed 14 articles $10.13,16,12,25,25,31,33.38$ with a total of 208 patients with Mahaim fibers and only 4 patients had atrial fibrillation as the presenting anthythmia $(1,9 \%)$. It is a much lower incidence as compared with the $10-32 \%$ incidence of atrial fibrillation in patients with the WPW syndrome ${ }^{10}$. Degeneration of circus movement tachycardia to atrial fibrillation was the mechanism in $25 \%$ of the patients with WPW studied by Bauenfend et at ${ }^{40}$. This mechanism may explain atrial fibrillation in the patient of Brugada et al ${ }^{36}$ who had an additional right posteroseptal $A P$ and antidromic tachycardia but could not explain atrial fibrillation in our patient who did not have other arrhythmias. The reason for the small incidence of atrial fibrillation in patients with Mahaim syndrome remains unknown but the presence of the Mahaim fiber itself seems to be important, as in the case reported by Miller et $a^{25}$, where atrial fibrillation could not be re-induced after ablation of the Mahaim fiber. 


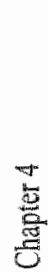

4
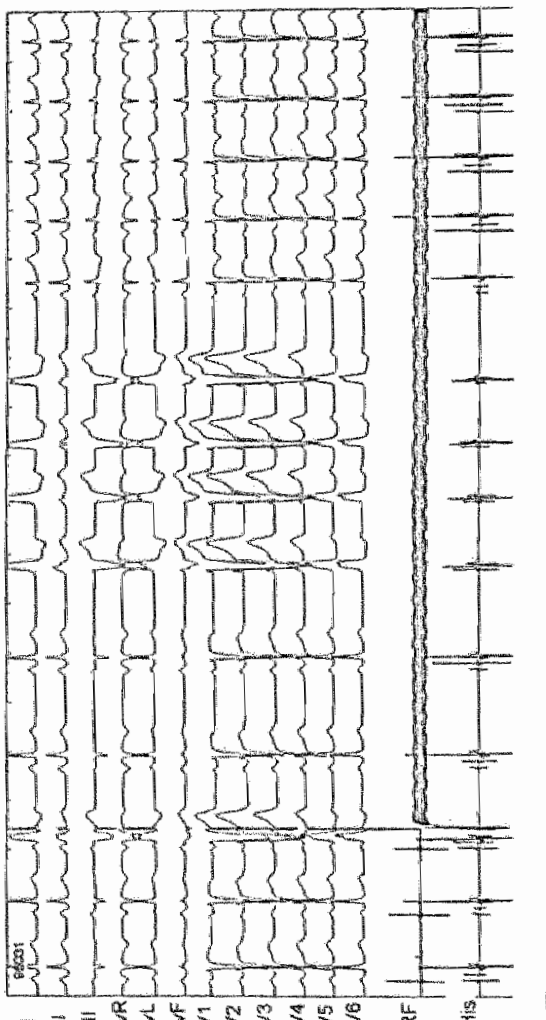

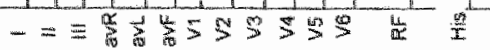

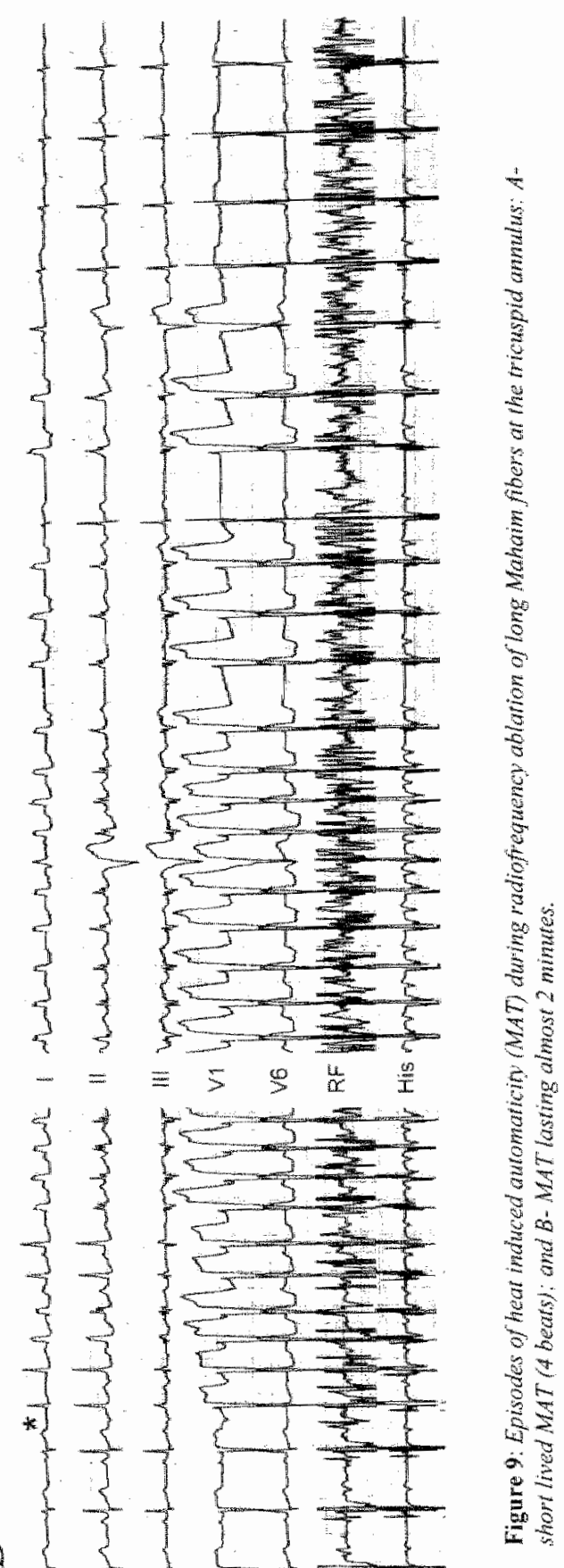




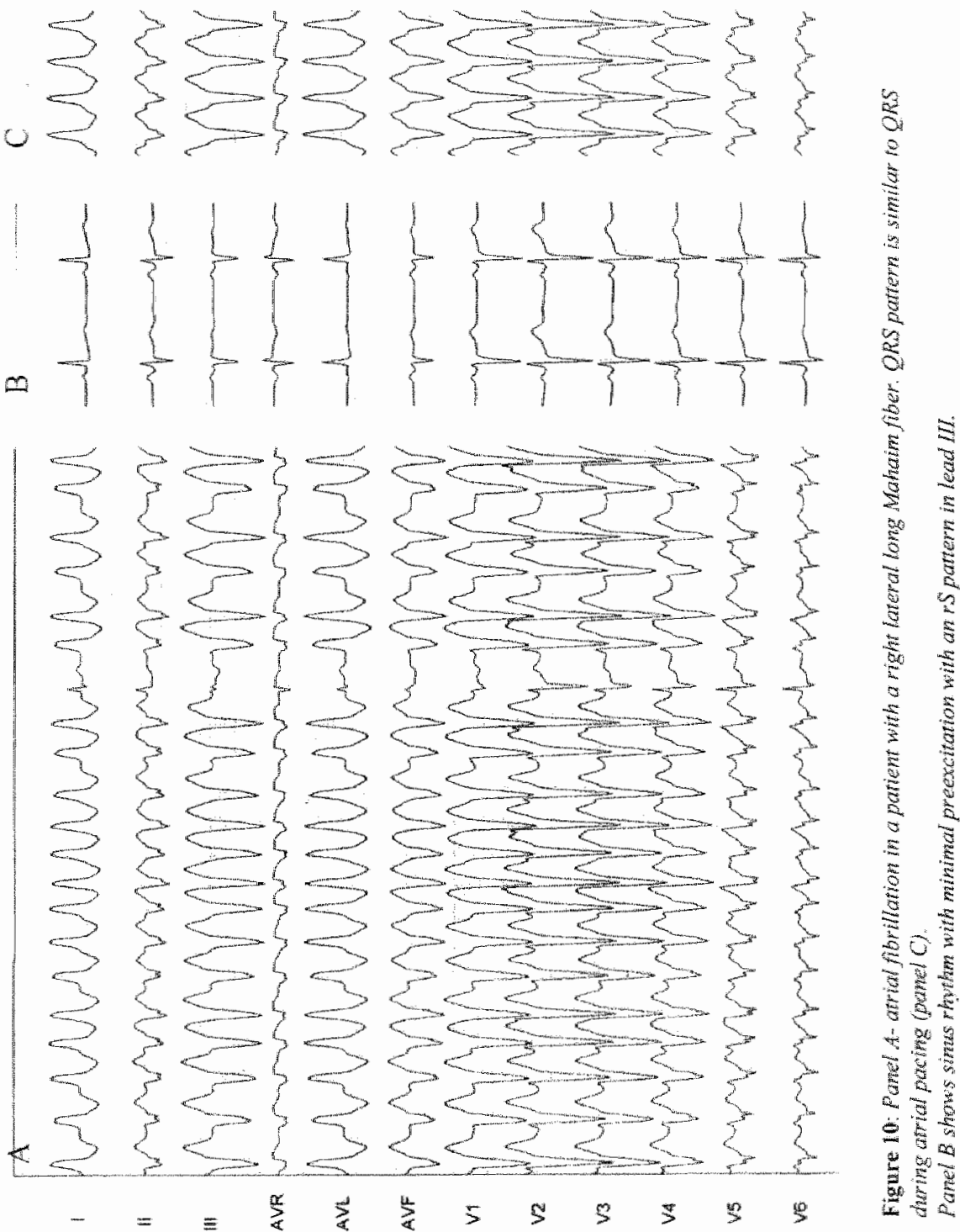




\section{NONREENTRANT PREEXCITED TACHYCARDIA DUE TO} SIMULTANEOUS DUAL CONDUCTION IN A MAHAMM FEER.

Nonteentrant supraventricular tachycardia with simultaneous conduction in the fast and slow A-V nodal pathways had been widely reported ${ }^{31}$. Those patients share some common features: they are usually refractory to antiarthythmic drug treatment. Tachycardia can be aggravated by the use of drugs; in the majority of patients no reentrant $A-V$ nodal tachycardia can be induced. The effective refractory period of the "fast" pathway is shorter than the "slow" pathway; they usually show absence of retrograde $\mathrm{V}-\mathrm{A}$ conduction. Without retrograde conduction over the "slow" pathway at the time of anterograde conduction over the "fast" pathway, conduction can proceed over the "slow" pathway and with a critical atrial cycle length it can reach the ventricle a second time. We have recently reported ${ }^{42}$ a patient with a Mahaim fiber showing likewise characteristics: an incessant tachycardia due to 1:2 P/QRS relationship (figure 1 i), no reentrant antidromic tachycardia could be induced, tachycardia did not respond to antiarhythmic drugs (sotalol and amiodarone) and he did not have $V-A$ conduction through the Mahaim fiber.

No further dual conduction occurred after successful catheter ablation of the decremental pathway.

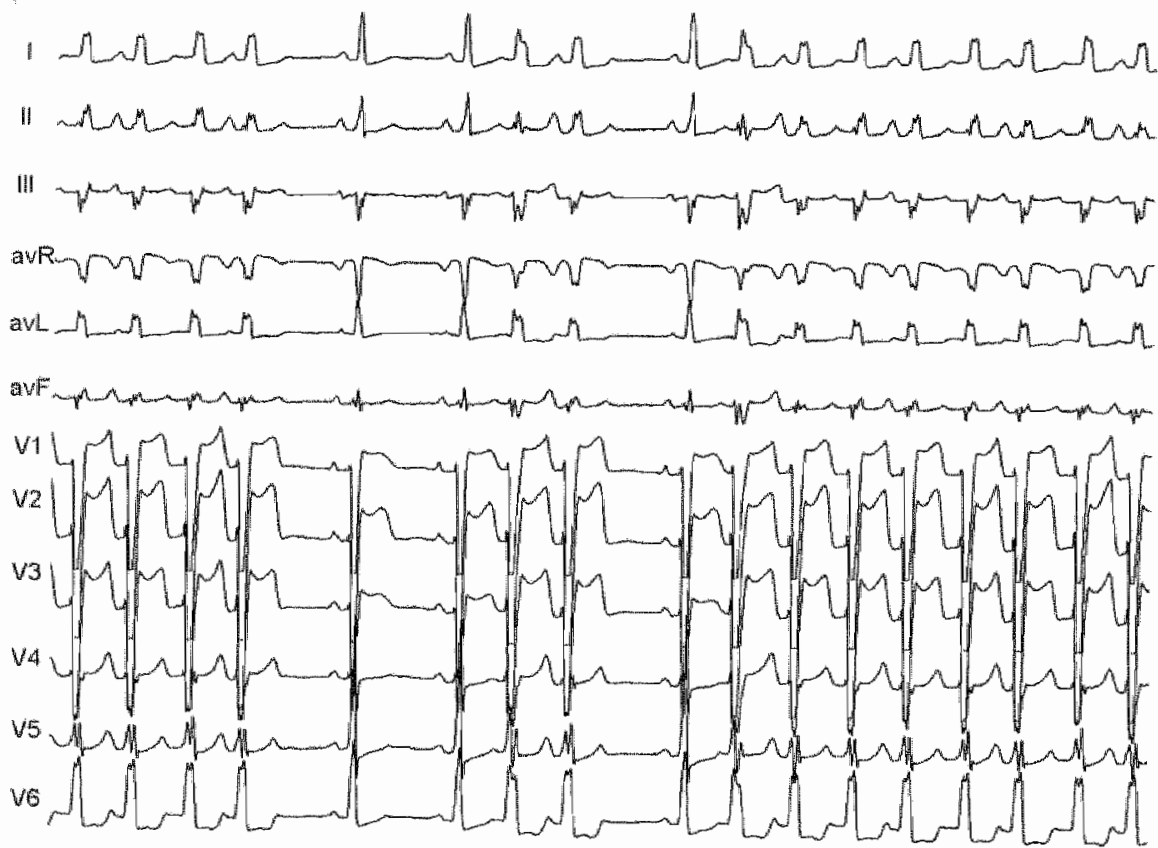

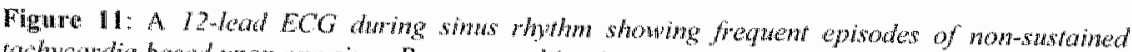

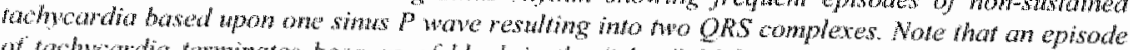

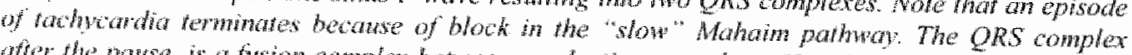

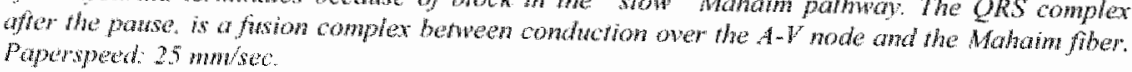




\section{ANTIDROMIC NODO-VENTRICULAR TACHYCARDIA WITH V-A BLOCK OR A-V DISSOCIATION}

Antidromic tachycardia with $\mathrm{A}-\mathrm{V}$ dissociation (or $2: 1 \mathrm{~V}-\mathrm{A}$ conduction) is the most important clue for diagnosing a nodoventricular fiber incorporated in the tachycardia circuit. Ventricular tachycardia is the main differential diagnosis of such tachycardia It is not possible to pre-excite ventricular activation, because the accessory pathway is taking off from the AV node. Some authors ${ }^{13}$ suggested that failure to pre-excite ventricular activation by atrial premature beats would be an argument for the presence of a NV Mahaim fiber. However, Porkolab et $a^{43}$ showed failure of decremental right atrial extrastimuli to reset an antidromic tachycardia in a patient with a true atriofaseicular Mahaim. This patient was successfully ablated targeting the Mahaim potential at the right lateral tricuspid annulus. In a patient with a LBBB-like tachycardia with $\mathrm{A}-\mathrm{V}$ dissociation, the presence of a true NV Mahaim fiber must be considered depending upon the finding of a progressive AH interval prolongation and a $\mathrm{HV}$ interval shortening during atrial pacing at increasing rates. Another clue is to induce a natrow QRS tachycardia with A-V dissociation in the same patient, due to reversal of the tachycardia circuit, with anterograde conduction over the $A-V$ node and retrograde conduction over the NV Mahaim fiber, as reported by Gallagher et alt.

\section{ORTHODROMIC TACHYCARDIA VIA A "CONCLALED" NF" NV FIBER}

There are very few cases with a NF Mahaim fiber and a clinical tachycardia where such a structure was diagnosed and ablated ${ }^{9-1 ?}$.

Those cases showed no pre-excitation at baseline ECG and a narrow QRS tachycardia with ventriculoatrial block or $A-V$ dissociation, and a midseptal concealed NF/ NV fiber was successfully ablated. Engagement of an accessory pathway was proven by interruption of the tachycardia or advancement of the next His bundle potential by giving a wentricular premature beat at the time of His bundle refractoriness. In one case reported by Hluchy et al ${ }^{2}$ there was $1: 1$ midline $\mathrm{V}-\mathrm{A}$ conduction and intermittent 2: $1 \mathrm{~A}-\mathrm{V}$ infra-Hisian block. Atrial tachycardia was ruled out because the taclycardia could be terminated by ventricular premature beats without $V-A$ conduction, and an accessory pathway polential preceded the earliest retrograde atrial activity, at the right midseptal area.

Shimizu at $\mathrm{al}^{\frac{44}{4}}$ reported a patient with Mahaim physiology and narrow QRS lachycardia preceded by a normal HV interval with $\mathrm{V}$-A dissociation. He could pre-excite $\mathrm{H}$ is bundle activation during tachycardia with a premature ventricular beat at a time of His bundle refractoriness. Atrial pacing findings were consistent with Maham physiology. The findings where suggestive of a nodoventricular Mahaim fiber.

12. ORTHODROMIC ATRIOVENTRICULAR TACHYCARDIA WITH A "BYSTANDER" FASCICULOVENTRICULAR PATHWAY

Fasciculoventricular pathways have so far not actively been associated with cardiac arrhythmias, but they are commonly associated with additional accessory 
pathways ${ }^{15}$. One has to be careful assessing the mechanism of any antidromic tachycardia before taking a decision to ablate. A "bystander" fasciculoventricular pathways can be misdiagnosed as a parahisian accessory pathway, while mapping during an orthodromic A-V reentrant tachycardia as shown in figure 12. This patient presented with a wide QRS tachycardia suggestive of an antidromic tachycardia using an anteroseptal bypass tract as the anterograde limb for a ventricular tachycardia). The baseline 12-lead ECG (figure $12 \mathrm{~A}$ ) was consistent

A
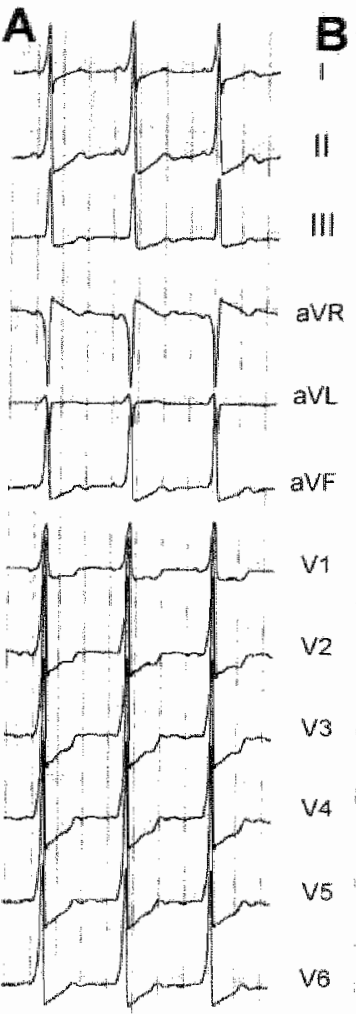

B

W
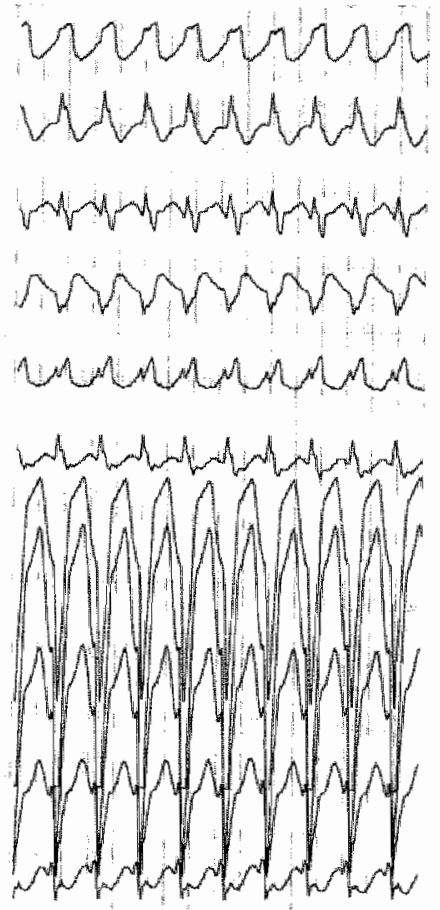
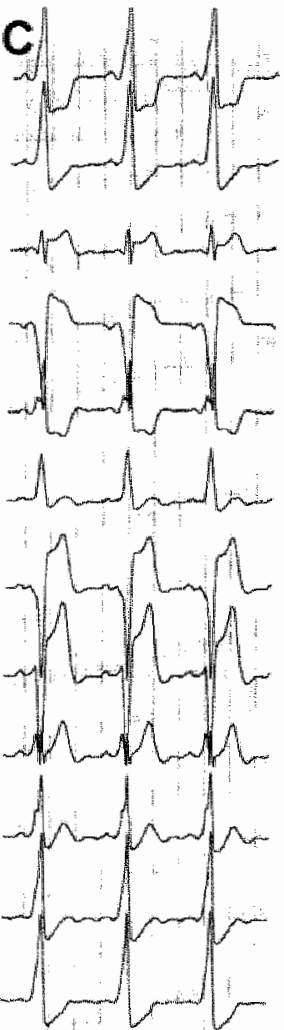

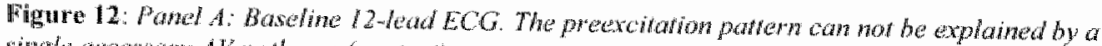

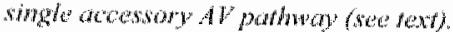

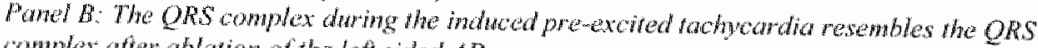
conplet afiem ablation of the lef shded AP

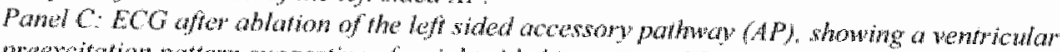

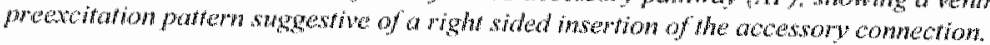

with a combination of two anterograde preexcitation patterns (a left lateral accessory pathway and a right-sided or anteroseptal accessory pathway). After 
ablation of the left-sided AP the ECG (figure 12 C) showed the same QRS pattern as during tachycardia. Electroplyysiologic findings included a fixed, short HV interval and preexcitation despite variation in the $\mathrm{AH}$ interval following atrial premature beats.
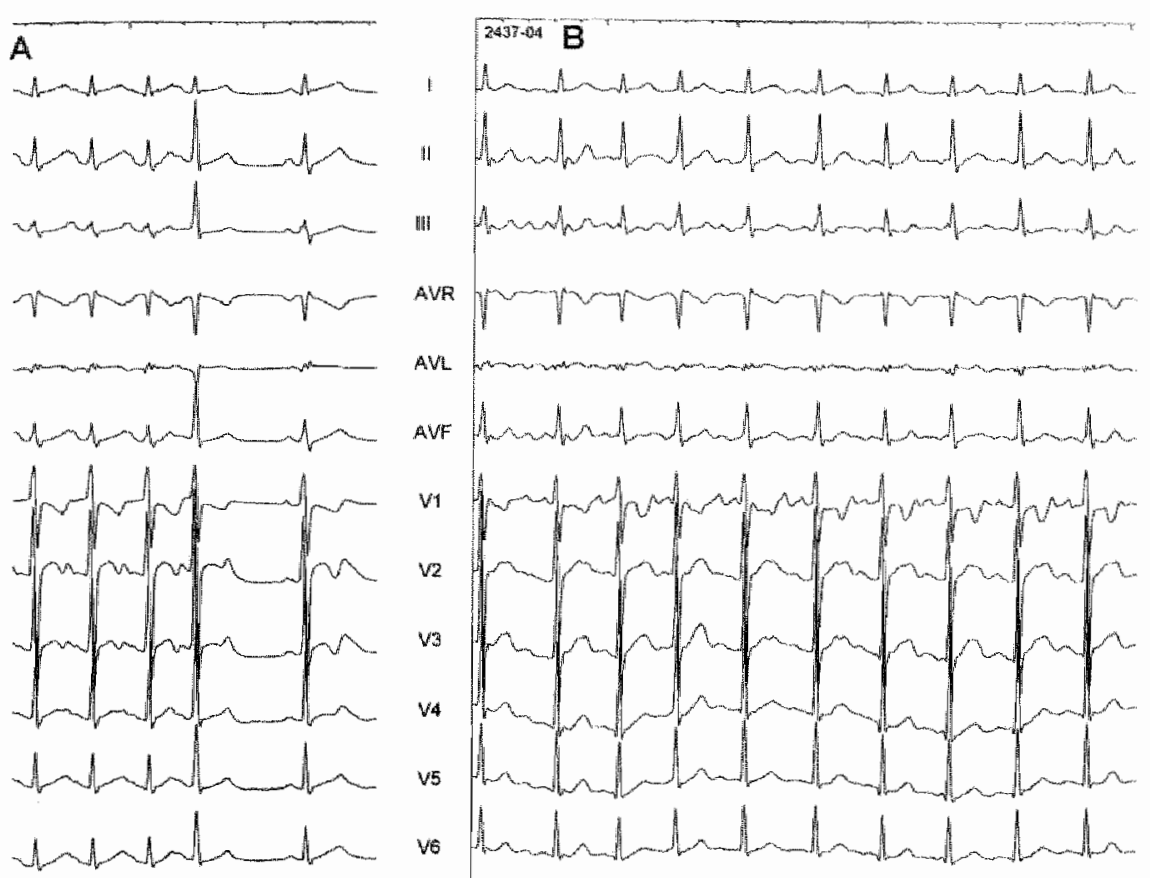

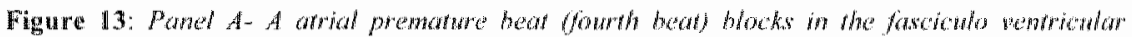

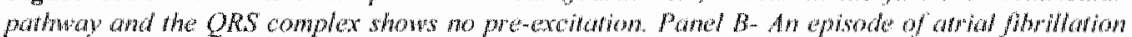

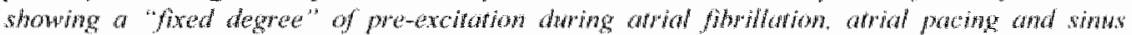
rhythm (pornet $A$ ).

One should suspect an additional fasciculoventricular pathway when the findings on the 12-lead ECG during simus rhythm can not be explained only by ventricular preexcilation over one accessory $A-V$ pathway.

\section{ATRIAL FIBRILLATION WITH ANTEROGRADE CONDUCTION OVER A FASCICULOVENTRICULAR PATHWAY}

Another clue to the diagnosis of a fasciculoventricular pathway can be seen in figure 14 which shows a self-terminating episode of atrial fibrillation with a minimally preexcited QRS complex. What strikes the eye is the absence of any 
variation in the degree of preexcitation both during changing $R-R$ intervals in atrial fibrillation and in sinus rhythm (figure 13). This is highly suggestive for an associated fasciculoventricular pathway. The association of atrial fibrillation with a fasciculoventricular pathway is fortuitous with no cause-effect relationship.

\section{CONCLUSION}

The large group of accessory pathways comprising the "Mahaim family" having diferent electrophysiologic characteristics and also diverse structural features can give rise to a number of reentrant and automatic rhythms. It is important to correctly understand their pathways in order to perform the correct therapeutic approach.

\section{References}

1. Meald $\mathrm{SC}$, Dawies W. Ward DE, Garrat $\mathrm{CJ}$, Rowland E: Radiofrequency catheter ablation of Maham tachycardia by targeting Maham potentials at the tricuspid annulus. Brit Heart I 1995;73:250-257.

2. Mahaim 1, Benati A: Nouvelles recherches sur les comexions superieures de la branche gauche du faiscealu de His-Tawara avec cloison interventriculaire. Cardiologia 1938;1:61-76.

3. Wellens HJ. Electrical Stimulation of the heart in the study and treatment of tachycardias. Thesis. Universiy Patk Press, Baltimone, 1971.

4. Gatlagher J, Smith WM, Kassell JH, et al: Role of Mahaim fibers in cardiac arthythmias in men. Circulation 1981;64:176-89.

5. Klein GJ, Guiraton GM, Kerr CR, at al: "Nodoventricular" accessory pathway: evidence for a distinct accessory atrioventricular pathway with atrioventricular node-like properties. I Am Coll Cardiol 1988:11:1035-1040.

6. Tchou P, Lehman MH, Jazayeri M, Akhtar M: Atriofascicular connection or an nodoventricular Mahaim fiber? Electrophysiologic elucidation of the pathway and associated reentrant circuit. Circulation $1988 ; 77: 837-48$.

7. Yamabe H. Okumuta K. Minoda K. Yastue H. Nodoventricular Mahaim fiber connecting to the left weniricle. Am Heart J 1991:122:232-234.

8. Tada H. Nogami A, Naito S. et al: Left posteroseptal Mahaim fiber asseciated with marked longindinal dissociation. Ibacing Clin Electrophysiol 1999:22:1696-1699.

9. Haissaguerre M, Campos I, Marcus Fl, et al Involventent of a nodofascicular connection in supraventricular lachycardia with VA dissociation: I Cardiowase Electrophysiol 1994,5:854 862 .

10. Grogin LR, Lec RJ, Kwasmun M, of al: Radiofrequency eatheter ablation of atriofascicular and modoventricular Mahaim tracts. Circulation 1994:90:272-281.

11. Huchy J, Schegehnitch P. Schichel $S$. et al: Radiofrequency ablation of a concealed nodoventicular Maham fiber guided by a discrete potential. I Cardiowase Electrophysiol
1099:10:603-610.

12. Hachy J, Schickel S, Worger URS, at al: Electrophysiologic aharacteristics and radiofrequency ablation of concealed nodofascicular and left anterograde atriofascioular pathways. I Cardiovase Electrophysiol 2000;11:211-217.

13. Kotkamp $H$. Hindricks $G$, Shenasat $H_{0}$ of all Variants of preexcitation-specialized atriolascicular pathways, nodofascicular pathways, and fisciculoventricular pathways: electrophysiologio findings and target sites for radiofrequency catheter ablation. J Cardiowase
Electrophysiol 1996:7:916-930.

14. Ganz L1, Elson J. Chenaricles MG: Preexcitation in a child with syncope. Where is the connchion? I Cardiovase Electrophysiol 1998,9:892-895.

15. Semick EB. Gerken LM Vrandecio M. Wellens HJI. Fasciculoventricular Pathways: Chinical and Elecirophysiologic Characteristics of a Variant of Prexexitation. I Candiovase
Electrophysiol 2003:14:1057-1063. 
16. Stemick EB, Tinmermans $\mathrm{C}$. Sosa $\mathrm{E}$, ef al The electrocardiogram duting sinus rhythm and tachycardia in patients whth anterograde conduction over Mahaim tibers: The importance of the "rS" pattern in lead I1.1. I Am Coll Cardiol 2004:44:1626 1635.

17. Haissaguerre $M$, Cauchemez $B$, Marcus $F$, et al: Characteristos of the ventricular insertion sites of accessory pathways with anterograde decrentental conducion propertiss. Circutation 1995;91:1077-1085.

18. Goldberger J, Pederson DN, Damle RS, el al. Antidromic tachycardia uthilizing decremental. latent accessory atrioventricular fibers: differentiation fron adenosine-sensitive ventricular tachycardia. I Am Coll Cardiol 1994:24:732.738.

19. Davidson NC, Morton JB, Sanders P, Kalman J. Latent Mahaim Iiber as a cause of antidnomic reciprocating tachycardia: recognition and successful radiofrequency ablation. I Candiovase Electrophysiol 2002:13:74-78.

20. Bardy GH, Fedor IM. German LD, et al: Surtace clectrocardiographic clues suggesting presence of a nodofascicular Mahaim fiber J Am Coll Cardiol 1984;3:1161-116\%.

21. Li HG, Klein GJ, Thakur $\mathbb{R K}$, Yee R: Radiofrequency ablation of decremental accessory pathways mimicking "nodoventricular" conduction. Am J Cardiol 1994:74:828-833.

22. Bardy $\mathrm{GH}_{\text {, German }} \mathrm{LD}$, Packer $\mathrm{DL}_{2}$ et al: Mechanism of tohycandia using a nodoventricular Mahain fiber. Am J Cardiol 1984;54:1140-1141.

23. De Ponti R, Stont C. Stanke A, et al: Radiofrequency catheter ablation in patients with Maham-type slow conduction accessory right atrio-ventricular pathway. Cardiologia $1994 ; 39: 169-180$.

24. Johna $\mathbb{R}$, Eckardt $\mathbb{L}$, Kirchof $P$. et al: Reproducible conversion from wide 10 nartow complex tachycardia: what is the mechanism? I Cardiovase Electrophysiol 2000;11:941-943.

25. Miller JM. Harper GR, Rothman SA. Hsia HH: Radiofrequency catheter ablation of an atrictascicular pathway during atrial fibrillation. A case report I Cardiowase Electrophysiol 1994;5:846-853.

26. Sosa E, Seanavacca M. Repetitive, non-sustained wide ORS complex lachycardia: what is the tachyeardia mechanism? I Cardiowase Electrophysiol 2001;12:977-8.

27. Sternick EB, Timmermans C, Sosa E, et al: Automaticity in Mahaim tibers. J Cardiovase Electrophysiol 2004; 15:738-744.

28. Sternick EB, Gerken LM, Vrandecic MO: Appraisal of "Mahaim" automatic tachycardia. J Cardiovase Electrophysiol 2002;13:244-249.

29. Thibault B, de Bakker. JMT, Hocini M, el al: Origin of heat induced accelerated jumctional thythm. J Cardiovasc Electrophysiol 1998:9:631-641.

30. McClelland $J H$, Wang $X$, Becknan $\mathrm{K}$. , et al Radiofrequency catheter anblation of right atriofascicular (Mahaim) aceessory pathways guided by accessory pathway activation potantials. Cireulation 1994:89:2655-66\%.

31. Bratan E, Siebbels J, Volkmer M, et al: Radiofrequency induced prexcited autonatic blythm during ablation accessory pathways with Mathaim-lype prexeitations does it predict clinical outcone? Pacing Clin Electrophysiol 1997,20:1121.

32. Dawies DW: Treatment of "Mahaim" tachycardiats by radioftequency catheict ablation. In Camm J, Lindemans FW (eds.): Transwerbous Defibrillation and Radiolfequency Ablation. Futurat Publishing Co., Ine., Armonk, NY 1995:199.208.

33. Ellenbogen KA, Ramirez NM. Packer DL. et al: Accessory nodoventricular (Mahaim) fibers. A clinical review. Pacing Clin Electropliysiol 1986:9:868-884.

34. Klein LS, Hacket K, Zipes DP. Miles WM: Radiolrequency catheter ablation of Maham fibers at the tricuspid annulus, Circulation 1993:87:738-747.

35. Bockeria LA, Chigogidze NA, Goluhhowa EZ, Artjukhina TV: Diwgnosis and surgien treatment of tachycardias in patients with nodoventricular fibers. Pacing Clin Lilectrophysiol $1991 ; 14: 2004-9$

36. Okishige $\mathrm{K}$, Friedman PL: New observations on decremental atriofascicular and nodofascicular fibers: implications for catheter ablation. Pacing Clin Electrophysiol 1995:18:986-998.

37. Mcclelland JH, Wang X, Beckman KJ. el al: Radiofrequency catheter ablation of right atriofascicular (Mahaim) accessory pathways guided by accessory pathway activation potentials. Circulation $1994,89: 2655 \cdot 66$.

38. Bragada J. Samohez JM. Kuzmicic B, et al: Radiofrequency calheter ablation of atriofascicular accessory pathways guided by discrete electrical potentials recorded at the micuspid annulus. Pacing Clin Electrophysiol 1995:18:1388-1394.

39. Campbel RWF, Smith RA, Gallagher Jj, et al: Arral fibrillation in the precexcitation syndirome. Ans f Cardiol $1977 ; 40: 514-520$. 


\section{Tachycardias associated with MAHAIM fibers: a review}

40. Batuefufeind RA, Wyndham CR, Swiry SP, et al : Paroxysmal atrial fibrillation in the WolfParkinson- White syndrome. Am J Cardiol 1981;47:562-569.

41. Csapo $G$. Paroxysmal nonreentrant tachycardia due to simultaneous conduction through dual atroventricular nodal pathways. An J Cardiol 1979;43:1033-1045.

42. Sternick EB, Sosia E, Scanavacca M, Wellens HJ. Dual Conduction in a Mahaim Faber. J Cardiovase Electrophysiol 2004, 15:1212-1215.

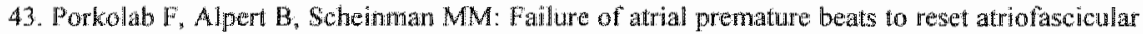
achycardia. Pacing Clin Electrophysiol 1999;22:528-530.

44. Shimian $A$, Ohe $T$, Takaki $H$, ef al: Narrow QRS complex taclizycardia with atrioventricular dissociation: Pacing Clin Electrophysiol 1988;11:384-393. 


\section{Chapter 5}

\section{The electrocardiogram during tachycardia in patients with anterograde conduction over a Mahaim fiber. Old criteria revisited.}

Eduardo Back Sternick*

Fernando E. S. Cruz Filhot

Carl Timmermans $\ddagger$

Eduardo A. Sosa"

Luz-Maria Rodriguez ${ }^{\ddagger}$

Luiz M. Gerken*

Márcio L. A. Fagundest

Hein J.J. Wellens ${ }^{\ddagger}$

From the Biocor Instituto*, Belo Horizonte, Brazil, Instituto Cardiologia Laranjeiras, Ministério da Saúde†, Rio de Janeiro, Brazil , University Hospitalł, Maastricht, The Netherlands, Instituto do Coração "da Faculdade de Medicina da Universidade de São Paulo, Brazil.

Heart Rhythm 2004;1:406-413 


\begin{abstract}
Background: Pre-excited tachycardia associated with decrementally conducting right sided accessory pathways usually shows a rather "narrow" QRS complex and can be difficult to differentiate from a supraventricular tachycardia (SVT) with LBBB aberrant conduction.

Methods and Results: In order to prospectively evaluate the sensitivity, specificity, positive and negative predicive value of previously described electrocardiographic critera to identify preexcited tachycardia due to decrementally conducting accessory pathways (QRS axis between 0 and $-75^{\circ}$, QRS width $\leq 0,15$ seconds, an $R$ wave in lead $i$, an $r S$ pattern in lead $V_{1}, R S>1$ QRS transition after $V_{4}$, and cycle length between 220 and 450 milliseconds), we analyzed three groups of patients: 32 patients with an atriofascicular pathway (group 1); 8 patients with a long $(\mathrm{n}=3)$ or short $(\mathrm{n}=5)$ decrementally conducting right sided atrioventricular pathway (group .I); and, a control group of 35 patients with SVT and LBBB (group III). Presence of all 6 criteria had a $87,5 \%$ sensitivity in group 1 and a $0 \%$ sensitivity in Group II. There were 4 false negatives in Group I. The negative predictive value was $82,5 \%$ with 6 false positives in group III (5 patients with an aberrant LBBB-shaped tachycardia with ventriculo-atrial conduction over an accessoly A-V pathway). The criterion cycle length was not helpful. Conchusions: Criteria for identifying a tachycardia with anterograde conduction over a Mahain fiber are only helpful in atriofascicular pathways with a sensitivity of $87,5 \%$ and a negative predictive value of $82,5 \%$. The major cause of false positives was a tacliycardia with aberant $\mathrm{LBBB}$ conduction and ventriculo-atrial conduction over an accessory A-V pathway.
\end{abstract}




\section{INTRODUCTION}

Twenty years ago Bardy et al ${ }^{1}$ reported six ECG features showing a high eflicacy in identifying antidromic tachycardia due to nodofascicular accessory pathways. In that study all $22 \mathrm{ECG}^{\prime}$ s with ventricular tachyoardia were correctly identified and only 1 of 18 ECG's with antidromic tachycardia was enoneously diagnosed as a Mahaim tachycardia. Those criteria - (1) a QRS axis between 0 and $-75^{\circ}$, (2) a QRS duration of 0,15 seconds or less, (3) an $R$ wave in limb lead 1 , (4) an $r S$ in precordial lead $V_{\|},(5)$ a transition in the precordial leads to a predominantly positive QRS complex after $V_{4}$, and (6) a tachycardia cycle length between 220 and 450 milliseconds - became the gold standard in identifying preaxited tachycardia due to anterograde conduction over a decrementally conducting accessory pathway. In the following decades our understanding about the anatomic course as well as the site of the proximal and distal insertion of such pathways has evolved ${ }^{2-1}$. Those pathways originally described as nodofascicular fibers would probably be re-classified today as atriofascicular pathways or decrementally conducting accessory atrioventricular pathways (figure 1). The am of our study was to evaluate the sensitivity, specificity and positive and negative predictive value of the criteria from the study of Bardy et all to identily patients with tachycardia due to atriofascicular and decrementally conducting accessory atrioventricular pathways with emphasis on the differential diagnosis with LBBB-shaped supraventricular tachycardia.

\section{METHODS}

\section{Common electrophysiologic findings}

Every patient showed during atrial pacing a decrementally conducting right sided atrio-ventricular bypass tract: progressive atrio-ventricular and $\mathrm{AH}$ interval prolongation coupled with a decreasing HV interval resulted in a greater degree of ventricular pre-excitation with a left bundle branch-like morphology". The His bundle deflection was inscribed after tine right bundle potential during maximal preexcitation. At maximal ventricular preexcitation, there was a constant QRS-His relationship without further changes on shortening the atrial pacing cycle length. A 12-lead ECG was stored simultaneously with intracavitary signals on a digital polygraph (MS or EP-TRACER, CardioTek BV, Maastriclat, The Netherlands).

\section{Definitions of terms}

An atriofascicular pathway showed a short $\mathrm{V}-\mathrm{H}$ interval, an early activation of the RV apex, and late activation at the annulus during pre-excited tachycardia. The atrial insertion was located by the finding of the accessory pathway ("M") potential (we did not differentiate the long atrioventricular decremental pathways inserting close to the distal right bundle area from the atriofascicular pathways). 

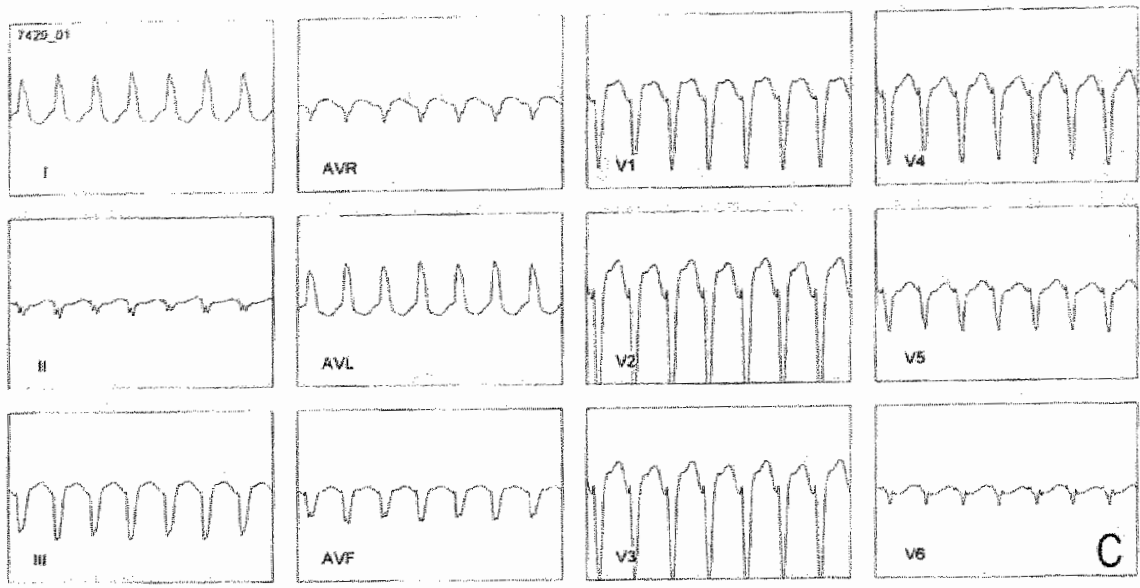

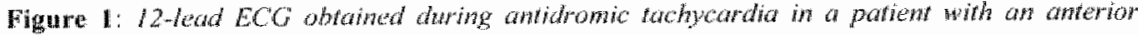
atriofascicular pothusy (ORS $1912 \mathrm{sec}$

Short clectemental atrioventricular pathways: during pre-excited tachycardia there was a long V-H interval, early ventricular activation was found at the tricuspid annulus, and late activation at the $R V$ apex.

Long decremental right superior atrioventricular pathways: during pre-excited tachycardia there was late activation of the right ventricular apex and late activation at the tricuspid annulus, and the $12-1 \mathrm{ead}$ ECG did not have left axis deviation (QRS frontal plane axis between +30 and +60 ). Atrial insertion was located by the finding of the " $\mathrm{M}$ " potential at the right superior area at the tricuspid anmulus.

Accessory pathway location nomenclature was presented in accordance to a previous expert consensus statement ${ }^{2}$.

We classified these patients with Mahaim fibers in 2 gronps: Group I: 32 patients with right sided atriofascicular pathways (figure 1); Group II: 5 patients with a short decrementally conducting right sided atroventricular pathway which was characterized by a distal insertion close to the tricuspid annulus (figure 2) plus 3 patients with long right superior decrementally conducting $A-V$ pathways figure 3). As a control group we examined 35 patients with SVT and aberrant conduction with a LBBB shaped QRS (Group III). All tracings were reviewed by the first author and 2 cardiologists to assess inter-observer variability.

The 40 consecutive patients with decrementally conducting accessory pathways came from 4 Institutions (tables I and 11). There were 23 females and 17 males, with a mean age of $25 \pm 13$ (range 8 to 80 ) years. Thirty-two patients underwent radiofrequency catheter ablation at the atrial aspect of the tricuspid anmulus where a discrete accessory pathway ("M") potential where recorded, while in 5 patients (cases $1,2,4,7$, and 8 - table I, AV Mahaim section) catheter ablation was carried out at the ventricular insertion guided by the pace-mapping technique. In 2 patients the pathway was surgically interrupted. 
TABLE I

Clinical data of patients with Mathaim fibers

\begin{tabular}{|c|c|c|c|c|c|c|c|}
\hline Case & Age & Gender & $\begin{array}{l}\text { Right sided } \\
\text { AFP site }\end{array}$ & C. Diagnosis & AP site & Ebstein & Therapy \\
\hline 1 & 32 & $F$ & Al & AVNRT & & & $\mathrm{RFp}$ \\
\hline 2 & 19 & $F$ & A & & & yes & $\mathrm{RFp}$ \\
\hline 3 & 21 & $F$ & Al & & & yes & $\mathrm{RFF}$ \\
\hline 4 & 13 & $M$ & A) & MPM & $\mathrm{PS}$ & ves & App \\
\hline 5 & 52 & $M$ & AI & & & & $\mathrm{RFp}$ \\
\hline 6 & 21 & $M$ & A! & & & & RFp \\
\hline 7 & 3 & $\mathrm{~F}$ & SA & & & & $R F p$ \\
\hline 8 & 30 & $M$ & Al & & & & $R F p$ \\
\hline 9 & 27 & $F$ & A & & & & $R F p$ \\
\hline 10 & 19 & $F$ & A & & & & $\mathrm{RFp}$ \\
\hline 11 & 12 & $F$ & Septal & & & & $\mathrm{RFp}$ \\
\hline 12 & 39 & $F$ & A & & & & $\operatorname{RFp}$ \\
\hline 13 & 15 & $M$ & $S A$ & & & & RFo \\
\hline 14 & 17 & $M$ & A & MPWCBT & PSIA & ves & $\mathrm{RFp}$ \\
\hline 15 & 23 & $F$ & $A_{1}$ & AVNNRT & & & $\mathrm{RFp}$ \\
\hline 16 & 80 & $M$ & Al & WPN & IPS & & RFp \\
\hline 17 & 25 & $\mathrm{~F}$ & Al & & & & $\mathrm{RFp}$ \\
\hline 18 & 35 & $M$ & A & & & & RFp \\
\hline 19 & 42 & $\mathrm{~F}$ & A & & & & $\mathrm{RF}$ \\
\hline 20 & 23 & F & A & & & & $R=p$ \\
\hline 21 & 23 & F & A & & & & RFp \\
\hline 22 & 45 & $F$ & A & & & & $\mathrm{RFp}$ \\
\hline 23 & 23 & $\mathrm{M}$ & A & & & & $s$ \\
\hline 24 & 11 & M & A & & & wes & RF-dis \\
\hline 25 & 26 & M & A & CBT & $\mathrm{P}$ & yes & RF-dis \\
\hline 26 & 26 & $\mathrm{~F}$ & A & AVNRT & & & RF.dis \\
\hline 27 & 31 & $\mathrm{~F}$ & A & & & & RF-dis \\
\hline 28 & 22 & $F$ & A & & & & RFp \\
\hline 29 & 17 & $M$ & I & & & & $\mathrm{RFp}$ \\
\hline 30 & 18 & $F$ & A & & & & RFp \\
\hline 31 & 31 & $F$ & A & & & & 5 \\
\hline 32 & 8 & $M$ & A & & & & RFp \\
\hline \multirow[t]{2}{*}{ Heane $\mathrm{SQ}=$} & $26+14$ & & & & & & \\
\hline & & & AVP site & & & & \\
\hline 1 & 31 & $M$ & $A$ & & & & $\mathrm{RFp}$ \\
\hline 2 & 12 & $\mathrm{~F}$ & A. & WPW & A & yes & RFp \\
\hline 3 & 45 & $\mathrm{M}$ & $S$ & & & & RFp \\
\hline 4 & 17 & $F$ & $A$ & & & & $\mathrm{PFP}$ \\
\hline 5 & 25 & $M$ & $\mathrm{~s}$ & & & & Pif.dis \\
\hline 6 & 19 & F & $s$ & & & & RFp: \\
\hline 7 & 50 & $F$ & $\mathrm{~A} . \|$ & & & & $R F p$ \\
\hline 8 & 25 & $M$ & A & & & & $\mathrm{RF}$ \\
\hline Wean $+S[D=$ & $24+10$ & & & & & & \\
\hline
\end{tabular}

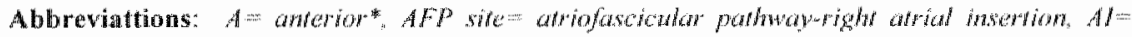

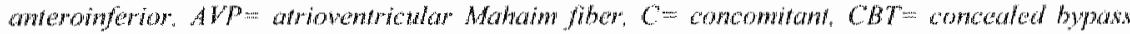
wact. $I=$ inferios. IPS $=$ infero paraseptal. $P=$ posterior. $S=$ superior, $S A=$ superoanfertor. Therapy: RFp= catheter ablation targeting the atrial insention guided by the proximat AP (M) potential. RFd = catheter ablation at the distal insertion of the Mathaim jober, $S=$ surgical endocardial abtation. "ESCWGANASPEP Expers Consensus Statentent 
The ECO during tachycardia in pathents with anterograde conduction over a Malhain fiber

Table II

Electrocardiographic characteristics of the pre-excited tachycardia

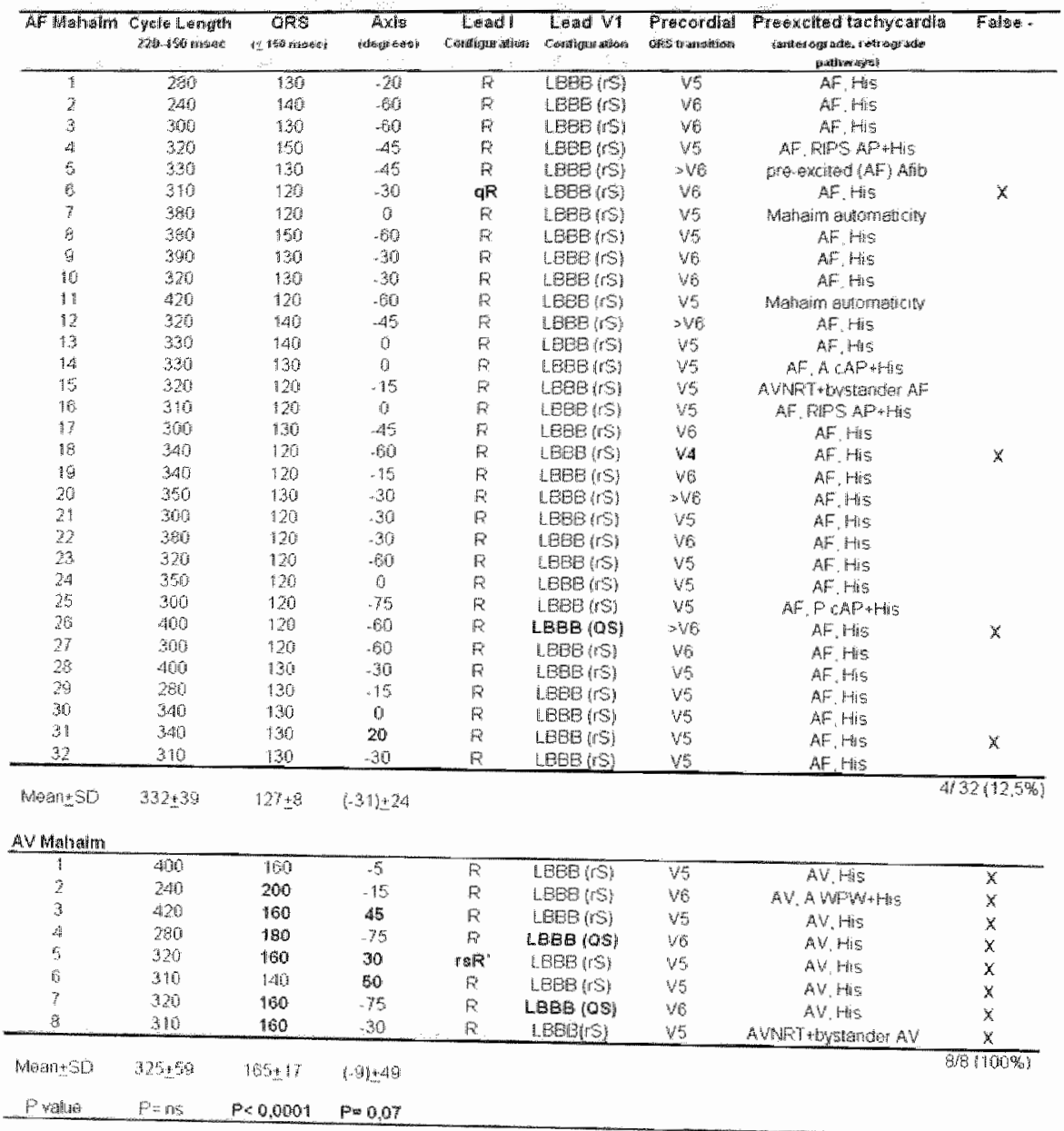

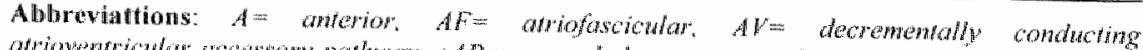

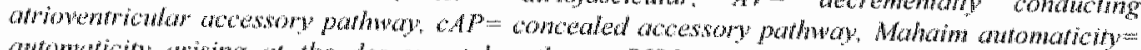

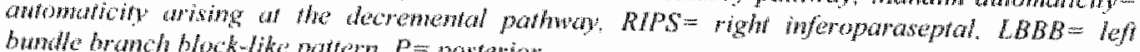

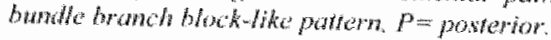


In all patients the radiofrequency catheter ablation or surgical intervention was strecessful. The control group consisted of 35 patients $(60 \%$ female) with left bundle branch block-shaped supraventricular tachycardia and a mean age of $40 \pm$ 20 years old (table III).

\section{Statistical Analysis}

Values are given as mean \pm standard deviation. The significance of differences ( $p$ $<0.05$ ) between groups of clinical and electrocardiographic parameters was assessed by Student's test or Fisher's exact test.

\section{RESULTS}

\section{Electrocardiogram during pre-excited tachycardia in patients with an} atriofascicular pathway (group I)

The index arrhythmia in the 32 patients with atriofascicular pathways was antidromic tachycardia with anterograde conduction over the atriofascicular pathway in 25 patients, incessant non-sustained spontaneous

automatic tachycardia arising at the atriofascicular pathway in 2 patients, preexcited tachycardia due to AVNRT with bystander anterograde atriofascicular conduction in one patient, pre-excited atrial fibrillation in one patient and antidromic tachycardia with anterograde conduction over the atriofascicular pathway and retrograde conduction with fusion between the A-V node and a rapidly conducting accessory pathway (two right inferior paraseptal and one anterior) in 3 patients. Those 3 patients with multiple accessory pathways also had an additional inducible orthodromice atrioventricular reentrant tachycardia. Mean cycle length of the pre-excited tachycardia was $332 \pm 39 \mathrm{msec}$ (range 220 to 420 ). Mean QRS width was $127 \pm 8$ (range 120 to 150 ) $\mathrm{msec}$. No patient with an atriofascicular pathway had a QRS larger than $150 \mathrm{msec}$. Mean QRS axis was -31 \pm 24 degrees (range 20 to -75 ), but only one patient with an atriofascicular pathway showed a positive frontal QRS axis during premexcited tachycardia. All patients with atriofascicular pathways showed an $R$ wave in lead I except case 6 (qR). Thirty-one patients showed an $\mathrm{rS}$ pattem in lead VI (case 26 had a $\mathrm{QS}$ pattern).

The QRS transition in the precordial leads $(R / S>1)$ occurred after $V 4$ in all but case 18 . Sensitivity of all 6 criteria in identifying an atriofascicular pathway was. $87,5 \%$ in these 32 patients. There were 4 false negatives.

Electrocardiogram during pre-excited tachycardia in patients with an atrioventricular accessory pathway with decremental conduction (group II)

The index arthythmia in 7 of 8 patients was an antidromic tachycardia using the Mahaim fiber as the anterograde limb of the circuit. In 6 patients the A- $V$ nodeHis bundle- right bundle branch system was used as the retrograde limb, while in case $2 \mathrm{~V}$-A conduction occured over the $\mathrm{A}-\mathrm{V}$ node and a right lateral rapidly conducting accessory pathway. One patient had an AVNRT with bystander anterograde conduction over a short atriowentricular Mahaim fiber (case 8). The mean tachycardia cycle length was $325 \pm 59(240$ to 420$)$ msec and within the prewiously described range. The mean QRS complex width $(165 \pm 17 \mathrm{msec}$ ) was larger than the width of the QRS in patients with atriofascicular pathways $\left(\mathrm{p}<0_{3}\right.$ 0001). Five of the seven patients had a QRS complex widh above the $150 \mathrm{msec}$ 


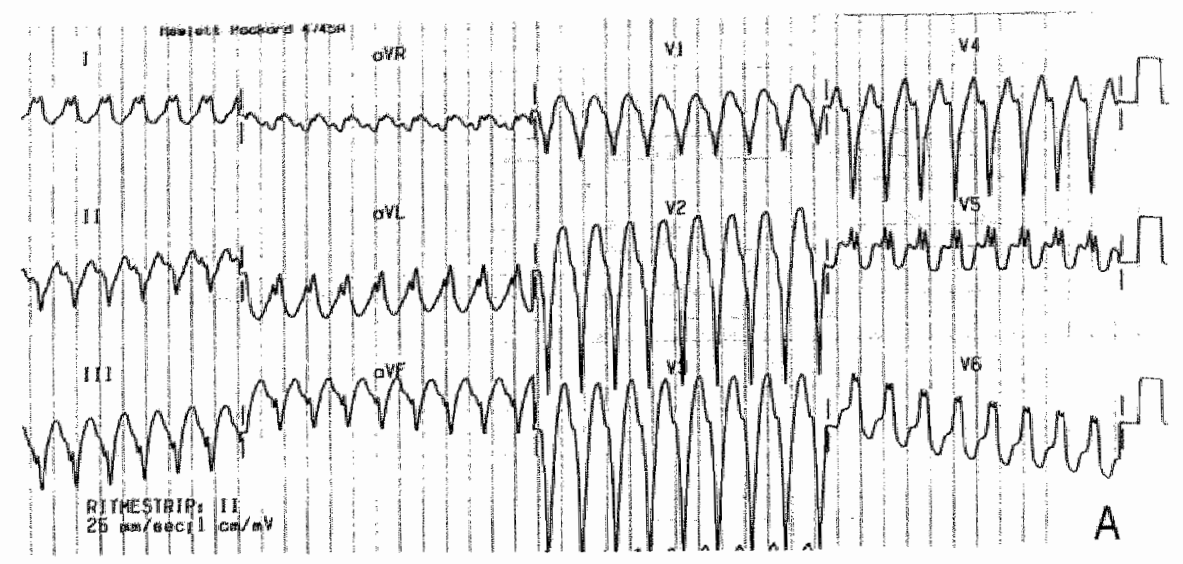

Figure 2: 12-tead ECG durimg antidromic tachycardia in a patien with a short decrementally atrionentriculas accessory parhwoy $(Q R S=0,17 \mathrm{sec}$.

limit. The mean frontal plane axis $(-9 \pm 49)$ in these patients were not significantly different $(p=0,07)$ from the

patients with an atriofascicular pethway in spite of a trend toward a less marked left axis deviation. Six patients showed an $\mathbf{R}$ wave in lead I and 6 patients showed an rS pattern in lead VI. No patient with an atrioventricular Mahaim would be diagnosed using the criteria from the study of Bardy et al. Those exclusions were based on the presence of 3 different criteria in 1 patient, 2 criteria in 3 patients and only 1 criterion in 4 patients. No exclusions occurred by the criterion "cycle length".

\section{Control group (group III)}

The control group consisting of patients with supraventricular tachycardia and LBBB abertant conduction had a similar gender distribution but a higher mean age $(40 \pm 20$ years $)(p<0,001)$. The tachycardia mechanisms were $A-V$ reentry due to an accessory pathway in 19 patients ( 14 left free-wall). AVNRT in 11 patients ( 1 slow-slow and 10 slow-fast) and atrial reentry in 5 patients. Mean tachycardia cycle length of $331 \pm 60$ msec was similar to the patients from Group I and II $(\mathrm{p}=\mathrm{ns})$. The mean QRS complex width $(153 \pm 23 \mathrm{msec})$ was also larger than the QRS width in patients with atriofascicular pathways $(p<0,0001)$ but not different from the group Il patients $(165 \pm 17 \mathrm{msec})(\mathrm{p}=\mathrm{ns})$. The patients with atrial reentry showed the widest QRS width $(188 \pm 18 \mathrm{msec})$. The mean frontal plane axis $(+10 \pm 49$ degrees $)$ in these patients was also significantly different 
Table III

Electrocardiographic characteristics of the LBBB-shaped $\mathrm{SVT}$

\begin{tabular}{|c|c|c|c|c|c|c|c|c|c|}
\hline 6 as: & 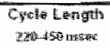 & 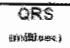 & 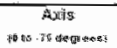 & $\begin{array}{c}\text { Lead } \\
\text { and }\end{array}$ & 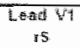 & 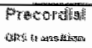 & 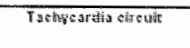 & Dfagetsen & Falat \\
\hline 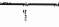 & 340 & 30 & $\sqrt{18}$ & retss & yes & Vis & सEIf & PEY & $x$ \\
\hline 2 & 320 & 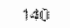 & 58 & ves & wes & w & 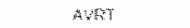 & my & \\
\hline 3 & $2 \times 40$ & 150 & 60 & ) & 的 & $n=$ & AVEF & Roston? & \\
\hline s & 240 & 160 & .10 & wes & no & $\sqrt{2}$ & 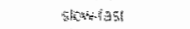 & muthe & \\
\hline 5 & 260 & 40 & .30 & $y \leqslant 5$ & 난 & $\sqrt{3}=$ & काint- & 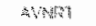 & $x$ \\
\hline 6 & 430 & 200 & 90 & mo [x? & no & 35 & 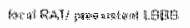 & 4 & \\
\hline$i$ & G:50 & 180 & 5it) & yes & W & $\forall 4$ & 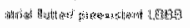 & Pandatitar & \\
\hline is & 360 & 170 & 4 & yes & $\%$ & $\mathrm{Fe}_{\mathrm{e}}$ & stotinst & सराशा & \\
\hline 9 & Mo: & 190 & $\theta$ & was: & no & 35 & An: & Fits & \\
\hline 10 & 340 & 1 40 & .60 & vos & no. & 45 & AnW $\mathrm{T}$ & Pका & \\
\hline 11 & 280 & 200 & $a$ & yes & 3no. & W & Nich igst & AUt Th & \\
\hline 12 & 260 & 160 & 60 & tos & no & $\sqrt{2}$ & AXRY & DET & \\
\hline 13 & $\$ 10$ & 140 & 75 & not & Wes & vo & RER & A mon & \\
\hline tat & 300 & $1: 40$ & 78 & nolat & yss & 40 & 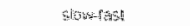 & 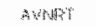 & \\
\hline 85 & 460 & 1,160 & 45 & ne trsh & yes & 45 & 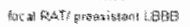 & A & \\
\hline 8 & 300 & 2900 & 15 & yes & หาo & ४ह & 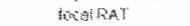 & MT & \\
\hline $1 \%$ & 350 & 17\% & .15 & yos & yes & VA & sllibetiagt & A.WW & \\
\hline 10 & 250 & 150 & 0 & was & noto & $1 s_{5}$ & swet ias? & Awat & \\
\hline 19 & 390 & 自80 & 60 & not & mo & Wa & AWT? & $130 \mathrm{dor}$ & \\
\hline 50 & 45 & 200 & 60 & $y_{12}$ & yos & $w i$ & (4) & 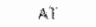 & \\
\hline $2+1$ & 320 & 180 & plo & $y+0$ & mo & 35 & Augr & PICt & \\
\hline 27 & 400 & 140 & .45 & ys & yes & $\sqrt{4}$ & AWT & $p$ wom & \\
\hline 22 & 320 & 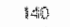 & Eิก & yes & we & $\sqrt{4}$ & Autror & purpind & \\
\hline 24 & 320 & $5 \div 0$ & 80 & no $\|_{R} \mathbb{R}^{2}$ & hes & vid & shatas & AWhrt & \\
\hline 25 & 200 & 160 & .89 & no $(9)$ & no & bs & saselani & HWA & \\
\hline $2 \hat{\mathrm{ki}}$ & 30 & $|\pi| a \mid 0$ & 60 & ye & 395 & VE & मीWT & $5 \mathrm{CHT}$ & \\
\hline 27 & 320 & 120 & $4 \mathrm{~B}$ & yes & yes & $\forall 4$ & silantial & 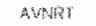 & \\
\hline 25 & 320 & Ha & 0 & 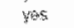 & Yos & vis & dut? & phent & $x$ \\
\hline 20 & 20 & 140 & 30 & was & 870: & $3 / 5$ & shong-sian & AVMFI & \\
\hline 30 & 30 & 190 & 60 & was & 50 & VA & A W & $\mathrm{PuBT}$ & \\
\hline 3 & 110 & 130 & 链 & nos & 405 & $\forall 5$ & whet & : CहT & $x$ \\
\hline 32 & 360 & 140 & 30 & yos & ves & 15 & $M_{4}+4 T$ & Fo WHP & \\
\hline 33 & 490 & 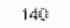 & 36 & yis & yes & $w$ & 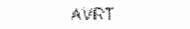 & PArWW & $x$ \\
\hline 34 & 320 & 130 & 33 & Yws & yos & $\forall s_{5}$ & $4 v a y$ & $\mathrm{PBT}$ & \\
\hline$t_{j}$ & 390 & 130 & 30 & $y$ & $y \theta s$ & 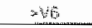 & Whipl? & $9 p \& Q$ & $x^{0}$ \\
\hline
\end{tabular}

Abreviattions: $A=$ anterior. $A P=$ accersory pathwa, AWNP = atrionentricular mode pathway,

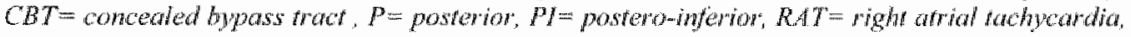
RIPS $=$ right inferoparaseptal, SPS $=$ superoparaseptal, WPW $=$ Wolff-Parkinson-White.

$(p<0,0001)$ from the axis in patients with an atriofascicular pathway, but not significantly different from group II patients. Six patients $(17 \%)$ would be erroneously (false positives) diagnosed as having a Mahaim tachycardia. The most frequent diagnosis associated with a false positive was the presence of an accessory pathway used for $\mathrm{V}-\mathrm{A}$ conduction. Five of the 19 patients with $\mathrm{A}-\mathrm{V}$ orthodromic tachycardia with aberrant LBBB (26\%) would be misclassilicd (figure 4). One of 11 patients (9\%) with AVNRT and aberrant LBBB would be classified as a tachycardia using an atriofascicular pathway. No patient with an atrial tachycardia due to atrial reentry was misciassified. Correct identification of aberrant SVT was done by 5 criteria in 1 patient, 4 in 2 patients, 3 in 7 patients, 2 in 10 patients and only 1 criterion in 9 patients.

\section{Inter-observer variability}

Comparative analysis of the 74 12-lead ECG's with $L$ BBB-shaped tachycardia by the 3 observers resulted in no disagreement. 


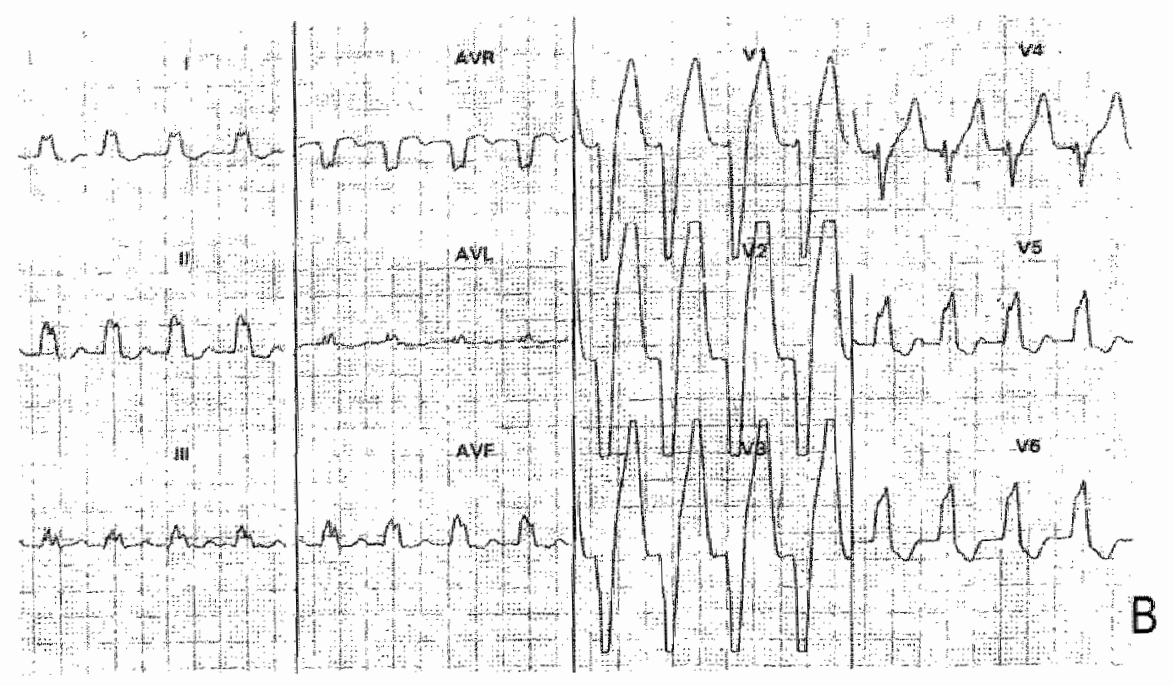

Figure 3: 12-4ead EOG daring anidrowic tachycardiat in a patient with a long decrementally accessury atromentricular pathway (QRS $=0.16 \mathrm{sec}$.

\section{DISCUSSION}

\section{Ventricular activation during a QRS with left bundle branch block configuration}

Our understanding of ventricular activation during left bundle branch block in humans is based on intraoperative epicardial studies ${ }^{13}$, catheter-based endocardial mapping studies ${ }^{14}$ and endocardial mapping study using a non-contact catheter technique ${ }^{15}$. These studies showed that in most patients with LBBB, activation started in the anterior RV wall. The delayed left septal activation (the septum is activated from the right to left side, and from an anterior to posterion direction) causes disappearance of the q wave in lead. 1 , aVL and in the left precordial leads and also of the wave in $\mathrm{Vl}$ in up to $50 \%$ of the patients. The activation of the anterior region of the $\mathrm{RV}$ can explain the inscription of a small and narrow $r$ wave in lead V $\mathrm{V}$ in the other half. In $97 \%$ of the group I patients with an atriofascicular pathway the presence of an $r$ wave in lead Vl is consistent with pre-excitation of the anterior region of the $\mathrm{RV}$, close to the area where the right bundle branch comnects to the RV myocardium. On the other hand, as expected, $37 \%$ of our patients with a LBBB-shaped SVT show a QS pattem in lead VL. In spite of a mean axis of $-31^{\circ}$ the wide range in frontal QRS axis from 0 to $-75^{\circ 7}$ can be explained by the quite variable site of early endocardial left ventricular septal activation in the presence of LBBB: high septal, postero-septal (close to the posterior fascicle or mid-septal ${ }^{\text {s. }}$. 

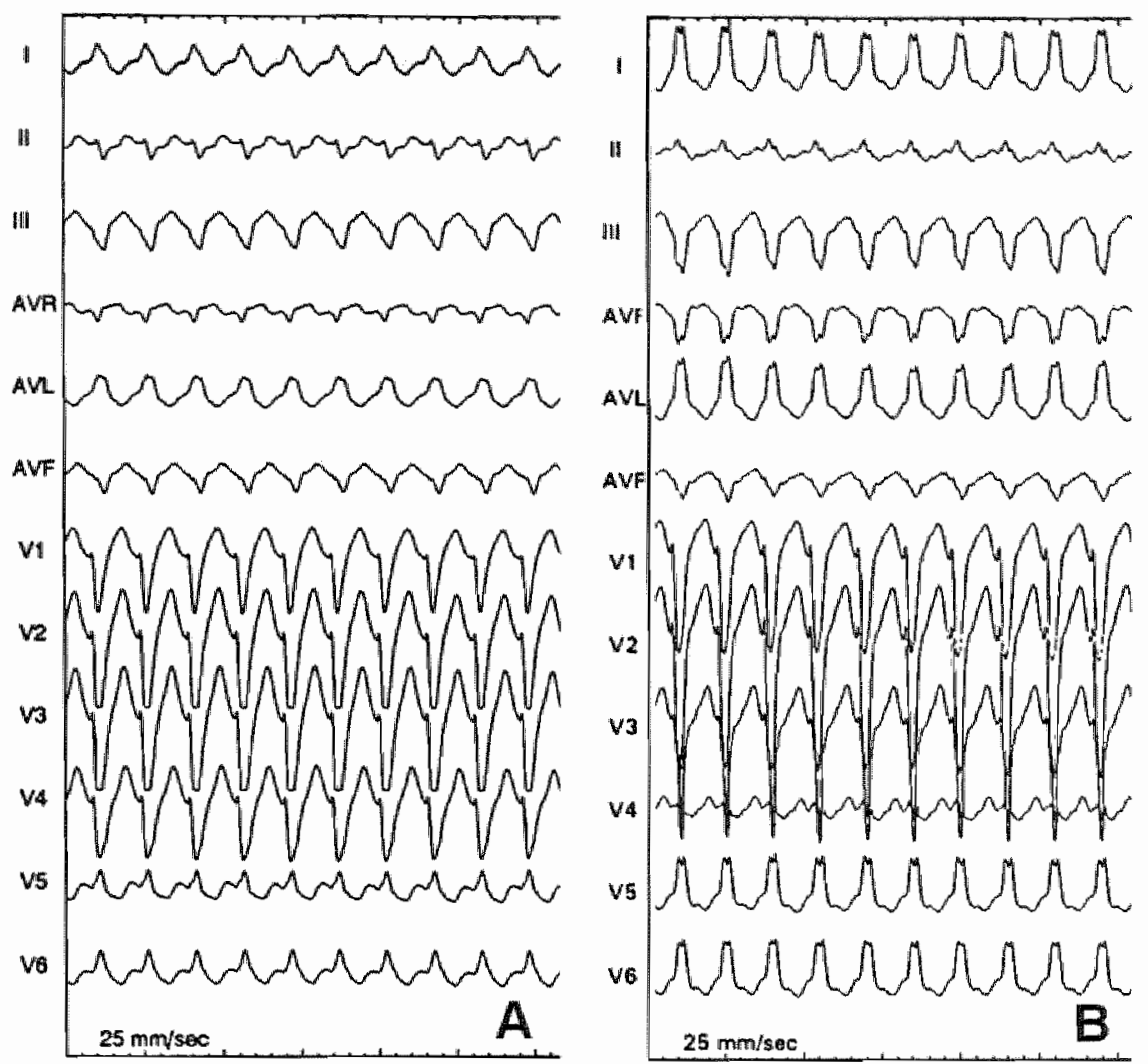

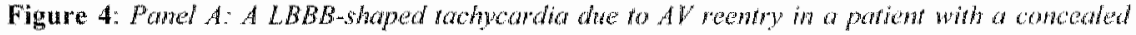

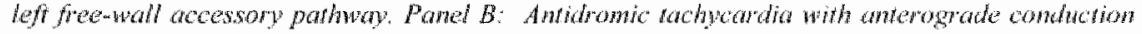

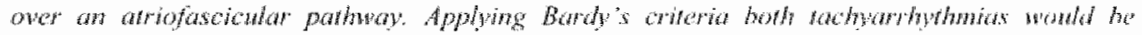
classifed as Maham tachyedrata.

LBBB tachycardia in patients with decrementally conducting accessory atrioventricular pathways

The causes of failure to identify these patients are lwofold: the absence of left ax is deviation in those 3 patients with long superior atrioventricular Mahaim's, whose distal insertion is not located in the vicinity of the distal right bundle branch, and the wider QRS width associated with a short decremental atrioventricular fiber, leading to a ventricular pre-excitation pattern like the one of a rapidly conducting right sided bypass tract. Six of the 8 patients had a QRS width of more than 150 msec.

\section{Aberrant LBBB-shaped supraventricular tachycardia}

The higher age of the control group can be explained by the inclusion of patients with atrial reentry and AVNRT which incidence peaks after the fouth decade. Our finding that 5 of the 6 false positives in the control group were caused by $A-V$ 
reentrant tachycardia using an accessory pathway highlights the importance of the tachycardia mechanism. As long as $26 \%$ of the $\amalg \mathrm{BBB}$ SVT due to an accessory pathway fall within the false positive range, a higher proportion of orthodromic tachycardia in the control group can decrease the negative predictive value of the aforementioned criteria, and vice-versa: the more patients with SVT and preexistent $\angle B B B$ are included the higher the positive predictive value, because this group of patients has a mean larger QRS width (188 $18 \mathrm{msec})$.

\section{Comparison of our data with the study of Bardy et al}

In spite of small differences between our data and the results reported by the group from Duke University, sensitivity $(87,5 \%$ \% $92 \%, p=0,9$ ) and negative predictive value $(82,5 \% \mathrm{ws} .91 \%, p=0,5)$ of the 6 electrocardiographic criteria did not reach statistical significance. The criteria cycle length was not helpful in this study as in Bardy's series.

All electrocardiographic criteria are simple, easy to assess, with no inter-observer variability and only the QRS transition in the precordial leads can be influenced by a mal-positioning of the electrodes, but that criterion would have the least impact on the results: I more false negative in group $I$ and 1 more false positive in group III.

\section{CONCLUSION}

The previously reported criteria showed reliable efficacy in identifying patients with an atriofascicular pathway, but are of no value in the diagnosis of a decrementally conducting accessory atrioventricular pathway. The tachycardia cycle length was not helpfull in making the correct diagnosis.

\section{References}

1. Barily GH, fedor JM, Gemmin LD, at al: Surtace electocardiographic cines sugyesting presence of an nofascicular Mahaim Liber. J Am Coll Cardiol 1984:3:1161-1168.

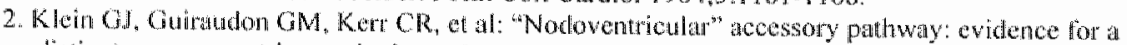
distinc accessory atroventricular pathway with athoventricular node-like properties. J Am Coll Cardion 1988:1:1035-1040.

3. Tchou P. Lehmetno MA. Jazayeri M, Akhtar M: Atriofascicular connection or a nodoventricular Mathatum fiber? Electrophysiologic atucidation of the pathway and associated teentrant circuit. Cincultion $1988 ; 77,837-48$

4. Guratalon CM, Guiraudon GM, Klein G. Histologic evidence for an accessory attoventicular pathway with $A$ Vanode-like morphology, Circulation $1988 ; 78$ (Suppl ID): 40.

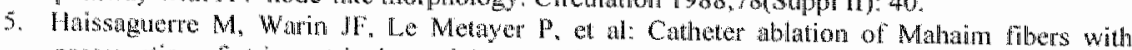
preservation of atriowentricular noda! conduction. Circulation 1990;82:418-427.

6. Klein L.S, Hacket FK, Zipes DP. Mites WM: Radiofrequency catheter ablation of Mahaim thbers at the tricuspid annulus. Circulation $1993 ; 87 ; 738-747$.

7. MoClelland JH, Wang X. Beckman KJ, et al: Radiofrequency catheter ablation of right atriofaschen (Mahain) accessory pathways guided by acessony pathway activation potontials. Circulation 1994:89:2655*66.

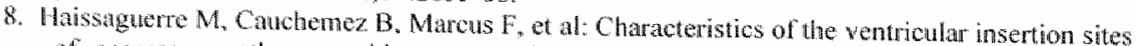
of acessony pathways with anterograde decremental conduntion properties. Circulation $1995,91: 1077-1085$

9. Cappato R. Schtuter M. Weiss C. ail: Catheter-induced mechatical conduction block of rightsidad atcessory hbers wh Mahaim-type preexcitation to guide radiofrequency ablation. 
10. Furg WHJ, Chan HCK, Chan WWL, Sanderson JE: Ablation of the Manwin Pathwa guded by noncontact mapsing. I Candiovase Electrophystol 2002; $13: 1064$.

11. Tan HL, Witkampf FHM, Nakagana H, Derksen R: Ariolascicular acessony pathway. I Cardiovas t: tectrophysio $2004,15: 118$.

12. Cosio FG, Anderson RH. Kuck KH, at. ESCWGANASPEP Experts consenus statumem. Living anatomy of the atrioventricular junctions. A guide to electroplysiologic mapping. I Cardiovase Electropinysiol 1999; 10:1162-1170.

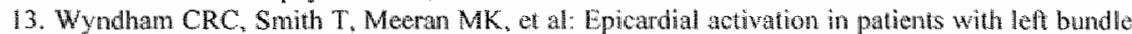
branch block. Circulation 1980:61:696-703.

14. Vassalo JA, Cassidy DM, Marchinshi at al: Findocardial aetivation of the lief bunde brench block. Circulation 1984;69:914-923.

15. Rodriguez LM, mimermans C, Nobar A, at al: Variabla patterms of septal auvation in patients with left bundle branch block and heart fallure. J Cardoxasc Electrophysiol $2003 ; 14: 135-141$. 


\title{
Chapter 6
}

\section{Fasciculoventricular fibers: clinical and electrophysiological characteristics of a variant of pre-excitation}

\author{
Eduardo Back Sternick* \\ Luiz M. Gerken* \\ Mário O. Vrandecic* \\ Hein J.J. Wellens ${ }^{\dagger}$
}

From the Department of Arrhythmia and Electrophysiology, Biocor Instituto*, Nova Lima, Brazil and the Interuniversity Cardiology Institute of The Netherlandst, Utrecht, The Netherlands.

Journal of Cardiovascular Electrophysiology 2003;14:1057-1063 


\begin{abstract}
Introduction: Fasciculoventricular tracts are considered a rare form of ventricular preexcitation. Few fasciculoventricular pathways have been reported, in none they have been linked to a reentrant tachycardia.

Methods and Reswlts: Four patients with fasciculoventricular bypass tracts underwent electrophysiologic evaluation. Two patients had a single fasciculoventricular pathway, one inserting anteroseptally and the other in the left ventricle. Two patients atso had an atrioventricular bypass tract, with anterograde conduction over the fasciculoventricular pathway during orthodromic atrioventricular reentrant tachycardia. After ablation of the atrio-ventricular pathways the ECG during sinus rhythm and the electrophysiologic study showed ventricular pre-excitation due to a fasciculoventricular bypass tract inserting into the right ventricle. Adenosine triphosphate was helpful in the diagnostic process.

Conchusion: Electrophysiologists should be able to make the differential diagnosis between a fasciculowentricular bypass tract and an anteroseptal accessory pathway to preclude potential harm to the $A-V$ conduction system if one targets a fasciculoventricular pathway for catheter ablation.
\end{abstract}




\section{INTRODUCTION}

Both fasciculoventricular and nodoventricular pathways are varieties of true Mahaim fibers.' 2. Since Gallagher et $\mathrm{al}^{3}$ published the largest series of fasciculoventricular pathways (6 patients) in 1981, few additional cases were reported ${ }^{4-6}$. A number of reasons account for the paucity of publications such as its ranity and underdiagnosis either because no cardiac arrhythmias occur or because of the small amount of ventricular preexcitation on the electrocardiogram. According to Joseplason ${ }^{7}$ it should be regarded as an electrocardiographic curiosity. However, in this era of therapentic cardiac electrophysiology, one should be able to differentiate an anteroseptal para-hissian bypass tract from a fasciculoventricular pathway, in particular in patients presenting with a tachycardia using an associated atrioventricular bypass tracts.

\section{PATIENTS AND METHODS}

We have seen 4 patients with right-sided fasciculoventricular pathways out of 332 patients with manifest ventricular preexcitation referred to our Laboratory for catheter ablation during the last 8 years $(1,2 \%)$. All four were male and without structural abnormalities of the heart on the transthoracic echocardiogram.

\section{Case reports}

Case I: A 13 year male patient with the WPW syndrome and recurrent paroxysmal tachycardia was referred for catheter ablation. The rest 12-lead ECG (figure 1A) was suggestive of a combination of two anterograde preexcitation patterns (a left lateral accessory pathway and a right-sided or anteroseptal accessory pathway). A pre-excited tachycardia, was induced during electrophysiologic study (figure (B). A right-sided bypass tract was thought to be responsible for anterogradle concluction of the electrical impulse during tachycardia. A left free wall bypass tract was used for ventriculoatrial conduction as shown by an eccentric left sided ventriculoatrial conduction pattern. Since it was not possible to advance ventricular activation by means of atrial premature beats during tachycardia we became suspicious of bystander anterograde conduction over an accessory pathway. We decided to ablate the left lateral bypass tract. Figure $1 \mathrm{C}$ shows the pattern of pre-excitation after radiofrequency current ablation of the left free wall pathway.

After ablation there was no ventriculoatrial conduction. The AH interval was 60 msec, the HV interval $25 \mathrm{msec}$. Incremental atrial pacing increased the $\mathrm{AH}$ interval with the development of $\mathrm{AV}$ nodal Wenckebach at a cycle length of 320 msec. The effective refractory period of the AV node was $260 \mathrm{msec}$. The amount of right-sided pre- excitation as well as the HV interval remained unchanged during atrial premature beats (figure 2).

Intravenous adenosine infusion caused transient complete A-V block without escape beats. No pre-excited QRS complex occurred till A-V conduction resumed. These findings are in agreement with a fasciculoventricular pathway inserting into the right ventricle and no further ablation was attempted. The patient remained asymptomatic during a follow-up of 3 years. 

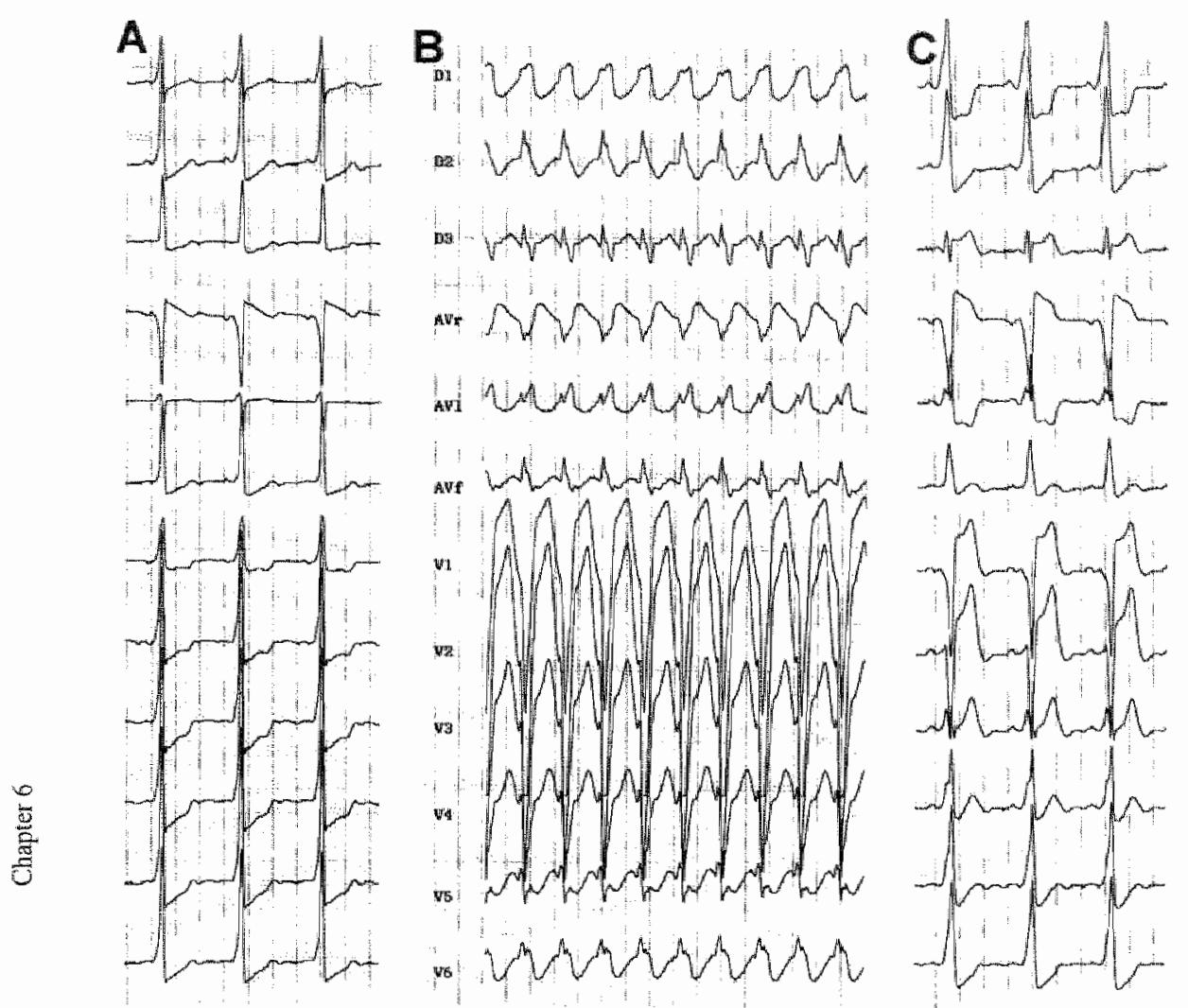

Figure 1: (Case 1) A: Baseline 12-lead ECG. The pre-excitation patien can not be explainad by a

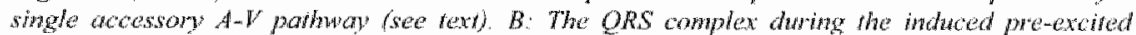

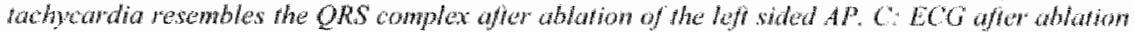

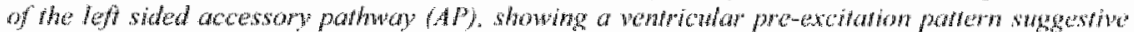
of a wh sted insertion of the acessory comberion.

Case 2: This 19 year male patient complained of palpitations but denied the occurrence of a sustaned tachycardia. The 12-lead ECC showed premextation with a P-delta interval of 0.12 sec. His general practitioner referred him for electrophysiologic evaluation. The delta wave was negative in $\mathrm{VI}$ and $+60^{\circ}$ in the frontal plane, consistent with an anteroseptal location of the accessory pathway (figure 3). The baseline $\mathrm{AH}$ interval was $50 \mathrm{msec}$, suggesting enhanced $\mathrm{A}$ - $V$ nodal conduction, but the $\mathrm{AH}$ conduction curve during incremental atrial pacing showed AH prolongation with an $A-V$ nodal Wenckebach conduction pattern at an atrial pacing cycle length of $380 \mathrm{msec}$. The IrV interval was sllort $(20 \mathrm{msec})$, did not change during atrial pacing, and showed a normal His bundlem right bundle activation sequence (figure 4). The amount of pre-excitation did not change during atrial pacing at increasing rates and induced atrial fibrillation (figure 3). 
Ventricular pacing during sinus rhythm showed ventriculoatrial conduction, which was concentric and decremental. Intravenous adenosine was administered in sinus thythm and during ventricular pacing. In both instances we observed complete conduction block. A few pre-excited escape beats were recorded with the same QRS configuration as during sinus thythm. No tachycardia could be induced by programmed stimulation. Mapping of ventricular activation during sinus rhythm showed the shortest delta- $V$ interval at the site of His bundle recording (figure 4). No ablation was attempted and the patient is arrhythmia free during a 6 -month follow-up.
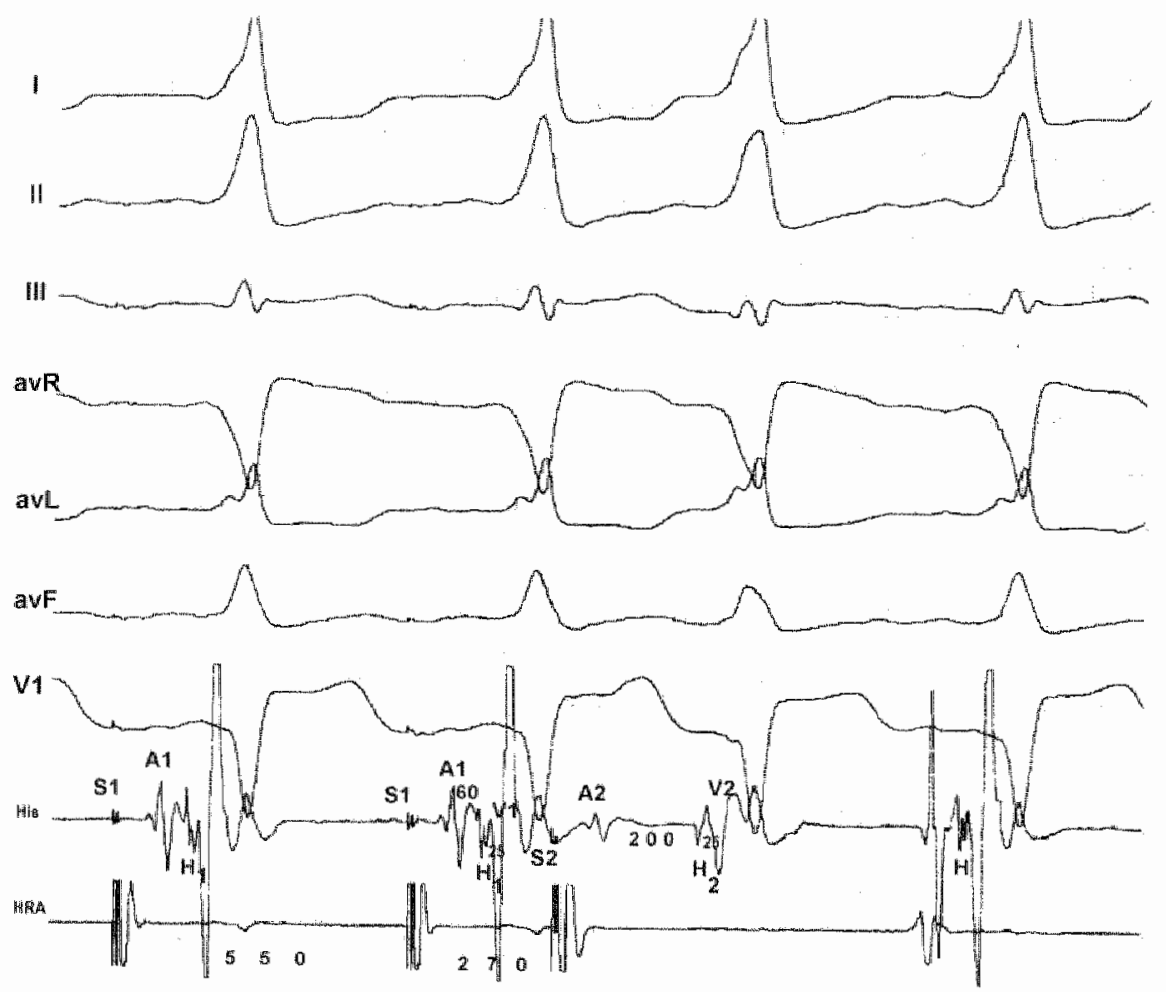

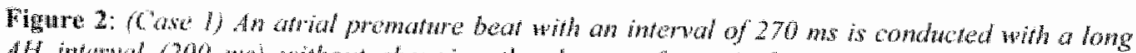
AH inferval (200) ms) without changing the degree of ventrowlar pre-encitation and the HW interval.

Case 3: A 19 year old male patient with the Wolff-Parkinson-White syndrome was admitted because of aborted sudden death while swimming. His sister underwent catheter ablation at our Institution 2 years before because of multiple accessory pathways. The patient had frequent episodes of paroxysmal tachycardia 
before his collapse. The baseline 12-lead ECG (figure 5A) showed a ventricular pre-excitation pattern that could not be explained by A-V conduction over a single accessory pathway, suggesting the presence of multiple accessory pathways. During electrophysiologic study a very fast antidromic A-V tachycardia was. induced by atrial extrastimuli (figure $5 \mathrm{~B}$ ). Ventriculoatrial conduction during tachycardia showed long conduction time and midline atrial activation. During ventricular pacing, however, ventriculoatrial conduction was lef sided. We decided to ablate this pathway, which was successfully accomplished by the transaortic approach and another pattern of ventricular pre-excitation emerged (figure 5C). A midseptal accessory AV bypass tract with bi-directional conduction was also successfully ablated at the base of the fasciculoventricular pathway

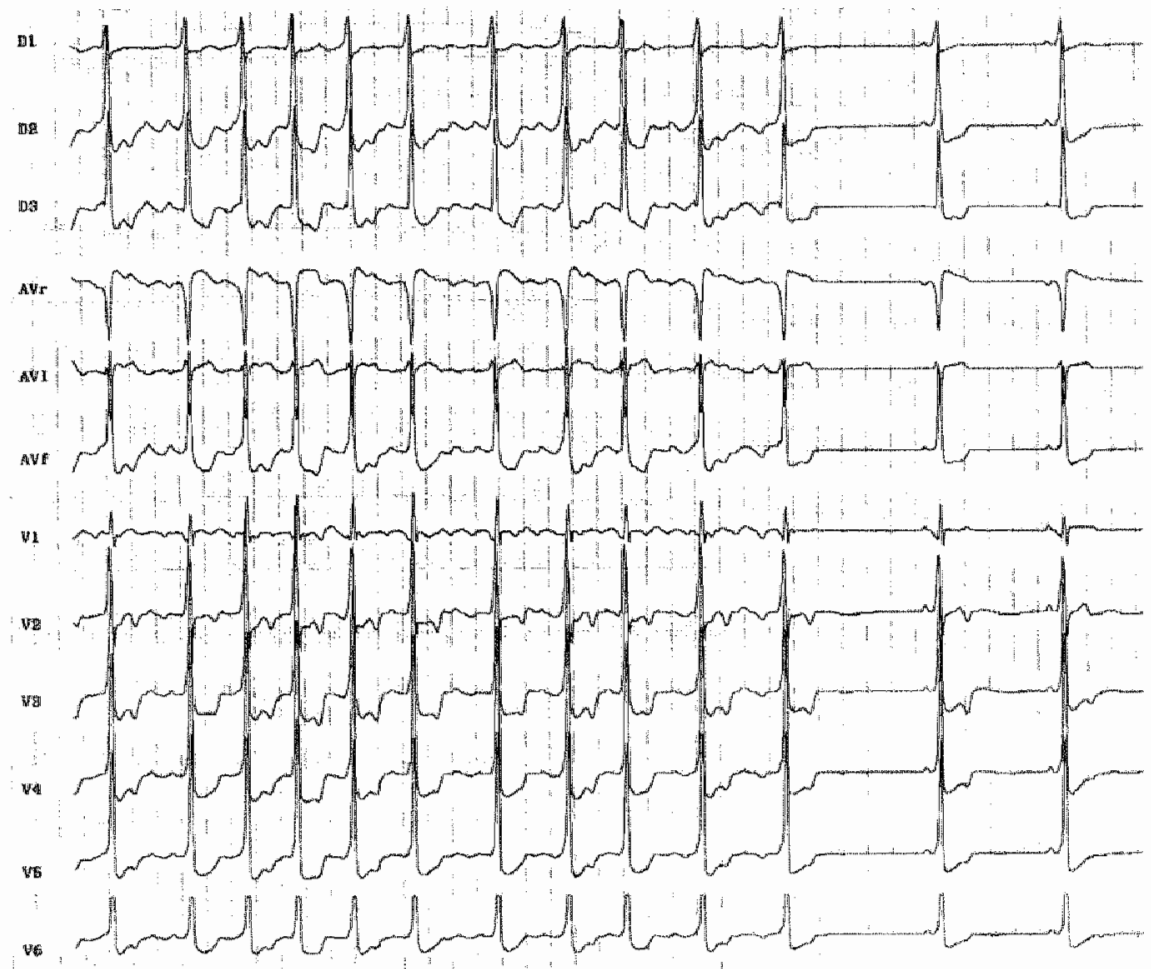

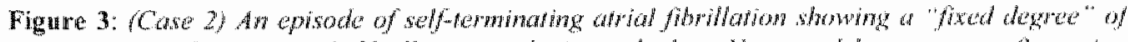

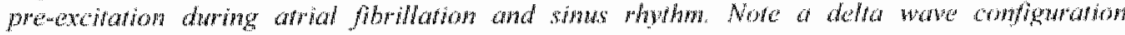
suggestive of an anteroseptat inserion of the accessory connection.

triangle of Koch.After ablation of those two AP, pre-excitation persisted but with a narrower QRS and a negative delta wave in $V I$ (figure 5D). Tachycardia could no longer be induced. The $A H$ interval was short but $A-V$ nodal conduction assessment showed a normal behavior during incremental atrial pacing and atrial premature stimuli. The HV interval was short and remained fixed during 
incremental atrial pacing as well as the amount of ventricular pre-excitation. Response 10 intravenous adenosine was $2: 1 \mathrm{~A}-\mathrm{V}$ block during simus rhythm without ary change in the QRS configuration. The diagnosis was made of a inserting in the right anteroseptal area. The patient is free of arrhythmias during a 1. -year follow-up.

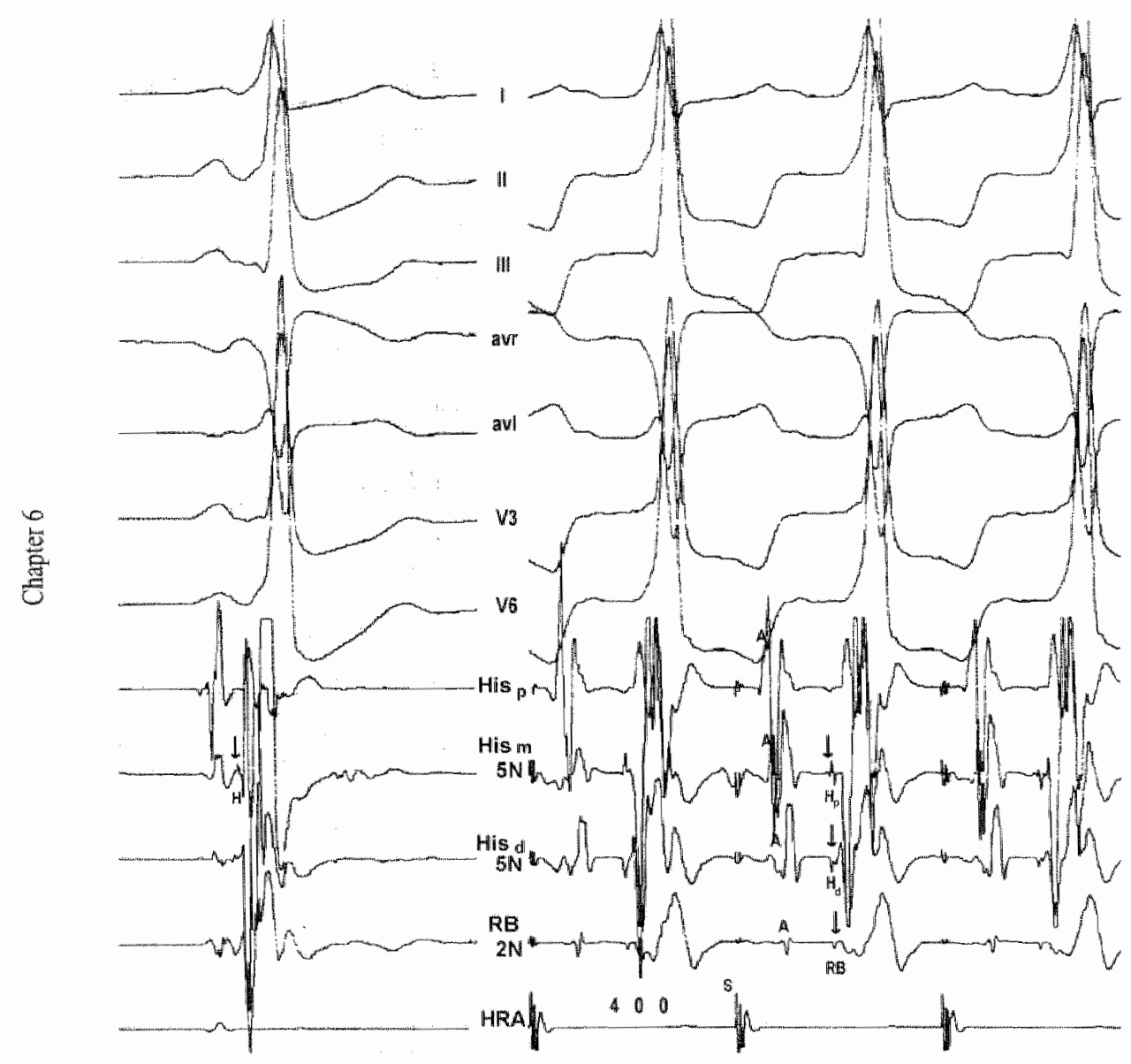

Figure 4: (Cave 2) Lefo panel: winns whym with pre-excitation. Right panel: High right arrial simudation tho moed showing prolongation of the AH interval wish a fixed HV interval and

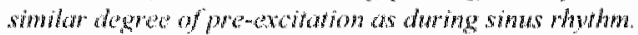

Case 4: A 51 year asymptomatic male patient was referred for electrophysiologic evaluation because ventricular preexcitation was found during a routine preoperative examination. The rest 12-lead ECG (tigure 6-A left panel) showed a short PR interval $(0,10$ sec) and minimal pre-excitation with a RBBB-like pattern suggestive of a left sided accessory pathway. Atrial activation pattem during right ventricular pacing was concentric with decremental V-A conduction. Incremental 
atrial pacing at the coronary sinus ostium resulted in prolongation of the $\mathrm{AH}$ interval but no change in the HV interval and the degree of preexcitation. Aurial premature beats with a coupling interval below $300 \mathrm{msec}$ showed a nomal $\mathrm{HV}_{\mathrm{V}}$ interval followed by a narrow QRS complex (figure 6-A right panel and figure 6-

A

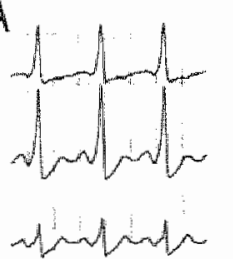

$\sqrt{1} \operatorname{lom}^{2}$

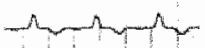

Naved

Hid
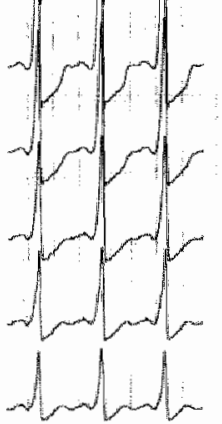

$B$

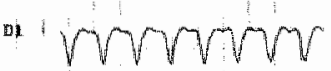

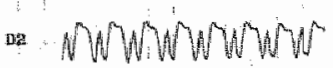

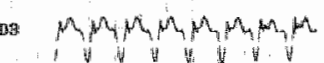

avr Moldnown
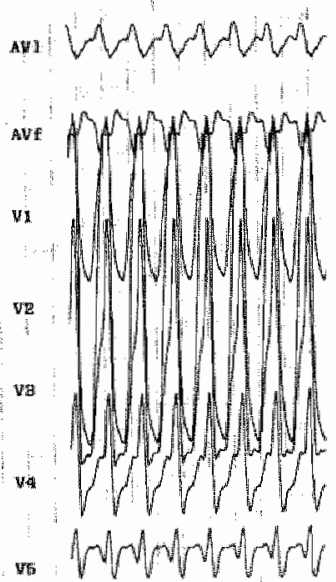

vo MWMWMW
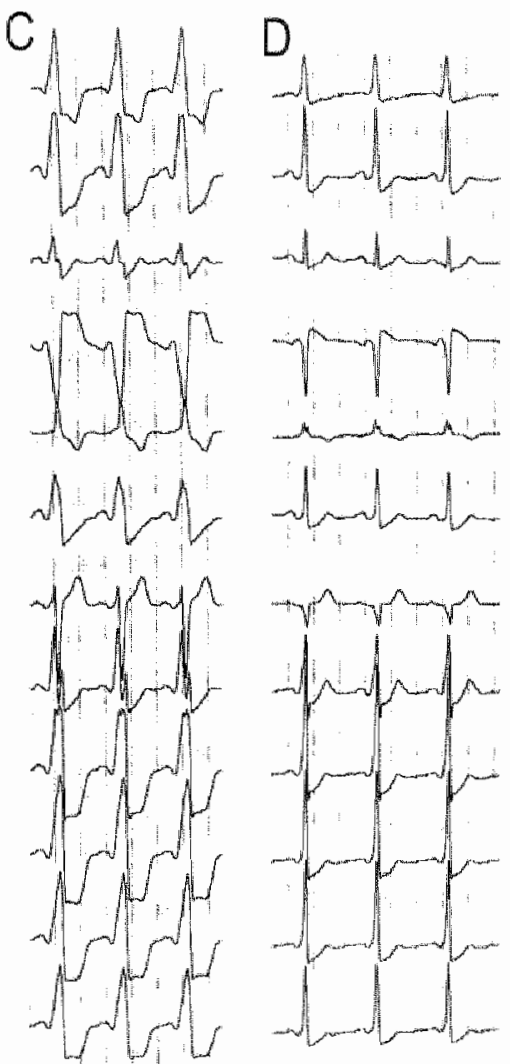

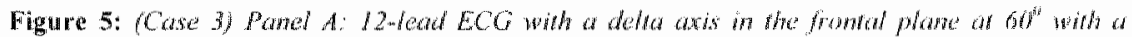

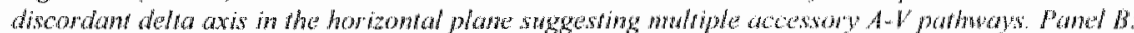

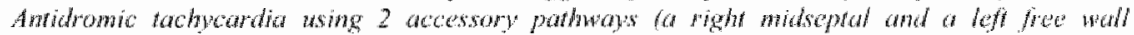

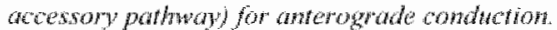

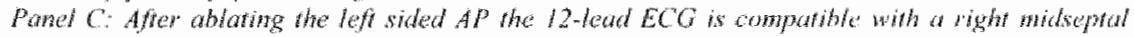
accessory paihnor.

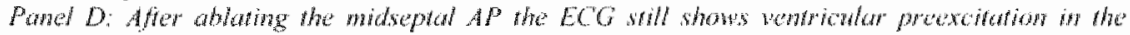
anerabeptal area bu with hess presexcination.

B). Those complexes were also seen as short $\mathrm{RR}$ intervals during induced atrial fibrillation. Intravenous adenosine (up to 36 micrograms) did not cause $\mathrm{A}-\mathrm{V}$ block. 
it resulted in sinus bradycardia and a junctional rhythm with a preexcited QRS complex. A fasciculoventricular pathway inserting in the left ventricle was the likely diagnosis and catheter ablation was not performed.

A
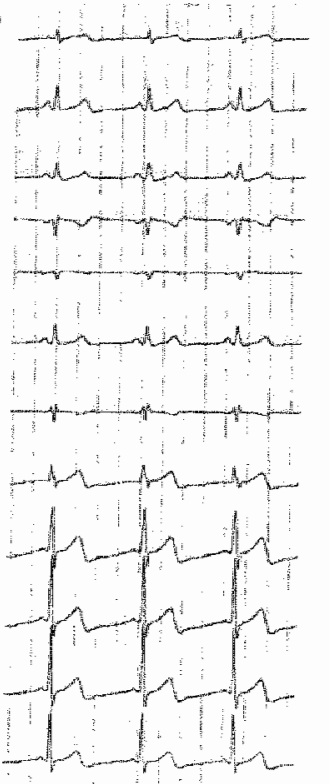

चु⿰耳

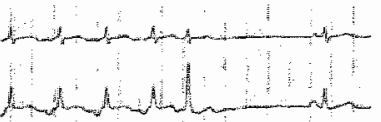

A9.

발

$+3$

Sit

蚁

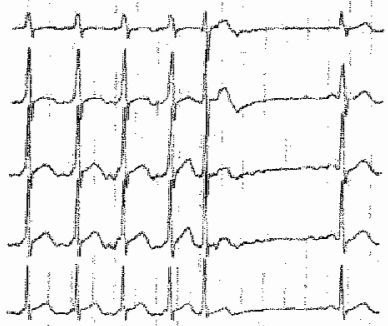

tat

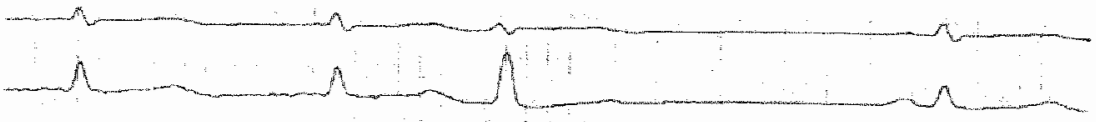

v․

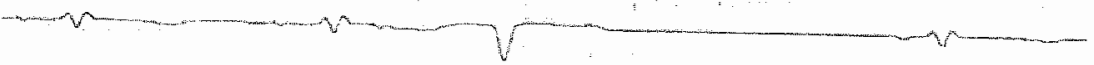

we

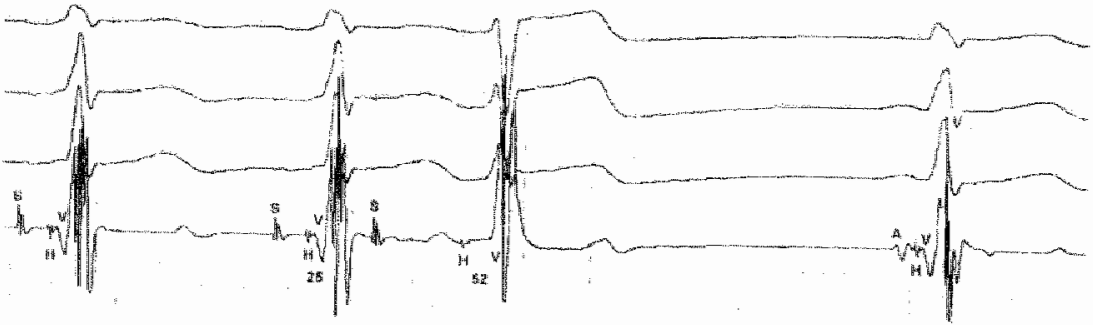

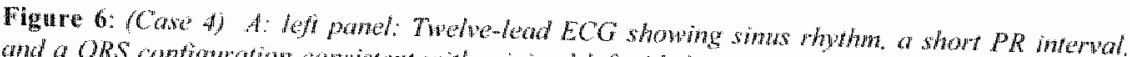

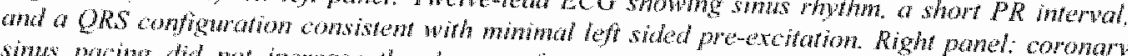

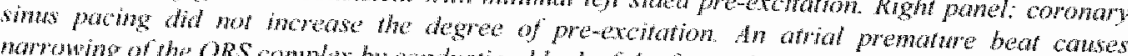

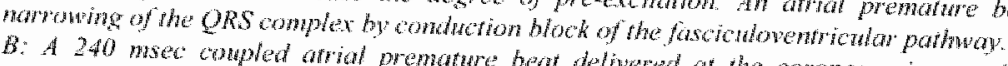

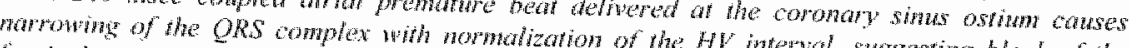

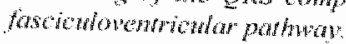




\section{DISCUSSION}

We found fasciculloventricular pathways in 4 out of $320(1,2 \%)$ consecutive patients referred for electrophysiologic study with ECGs showing ventricular preexcitation. Previous reports in predominanty adult populations gave simiar figures: $6(1,9 \%)$ out of 308 patients $^{3}$ and $2(1,8 \%)$ out of 111 patient $s^{7}$.

Enhanced $\mathrm{A}-\mathrm{V}$ nodal conduction was a common finding among the patients studied by Gallagher et al ${ }^{3}$. However, ow 4 cases, in spite of having a short intranodal conduction time (AF interval) during sinus rhythm, showed nomal $\mathrm{AH}$ interval lengthening and $\mathrm{A}-\mathrm{V}$ nodal behavior during incremental atrial pacing.

The classic findings of a fascicaloventricular connection include prolongation of the $A H$ interval without changes in the HV interval, a constant degree of preexcitation, and a fixed relationship between the His bundle and the right bundle potential, during incremental atrial pacing. This was found in all our 4 patients.

We found that intravenous adenosine was very useful as a method to assess the relationship between the fasciculoventricular pathway and its connection to the A$V$ node-His bundle system. The use of adenosine triphosphate as a diagnostic tool is based on its transient marked negative $A-V$ nodal dromotropic effects". In addition, adenosine shortens the action potential duration of an accessory pathway ${ }^{\text {ing }}$.

Adenosine did not elicit a greater degree of pre-excitation in any of our patients, but created second degree or complete $A-V$ block with junctional beats with the same degree of pre-excitation and short HV interval as during sinus rhythm. His bundle pacing producing an identical QRS as during sinus rhythm is another method to demonstrate the presence of a fasciculoventricular fiber. However, we found it difficult to get relliable and stable tis bundle pacing. Two out of our 4 cases had associated bypass tracts and circus movement tachycardia. Gallagher et al ${ }^{3}$ reported associated WPW syndrome in 1 out of 6 patients, Sallee III et al found multiple accessory pathways in 1 out of his 3 children, and the patient reported by Kotkamp et al ${ }^{4}$ also had a left lateral bypass tract. The child reported by Ganz et al did not have associated WPW. Taking all these patients withfasciculoventricular pathways into account, including ours, the incidence of associated WPW would be $33 \%$ (5 out of 15 patients). Multiple accessory pallways are estimated to occur in up to $13 \%$ of WP W patients". Higher figures. may represent bias due to referral of symptomatic patients. Nonetheless, it is important to recognize in patients with ventricular pre-excitation referred for catheter ablation, that a fasciculoventricular pathway and not an anteroseptal bypass tract is present, avoiding damage to the $\mathrm{AV}$ node-His bundle system if such pathway is mistakenly targeted for catheter ablation.

\section{CONCLUSION}

One should be especially suspicious for an additional fasciculoventricular pathway when the findings on the 12-lead ECG during sinus rhythm can not be explained by ventricular pre-excitation over one accessory A.V pathway only. We ablated the left-sided pathways first (cases 1 and 3) as well as the midseptal accessory pathway (case 3) after having obtained electrophysiologic data supporting their location and their active role during tachycardia. After ablation of these pathways the remaining pre-excitation should be assessed carefully. 


\section{References}

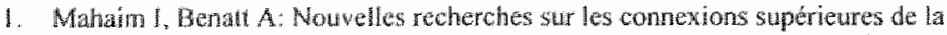
prancli gache du Cáscea de His-Tawara aves cloison interventriculaire. Cardiologia $1938: 1: 61-76$

2. Wellens HII. Electrical stimulation of the heart in the study and treatment of tachycardias. Universily Park Press, Balimork, 1971:70-121.

3. Galloghen J, Smith WM, Kasell JH, al al: Role of Wahaim hbers in cardiac arthythmias in man. Circulation $1981 ; 64: 176-189$.

4. Kothamp H, Hridricks $G$, Shenasa $H$, at al: Variants of prexcitation-specialized arrofascichlar pathways, nodofascicular pathways, and fasciculoventricular pathways: electrophysiologic finding and target sites for radiofrequency catheter ablation. J Cardiovasc Electrophysiol 1996:7.916-930.

5. Ganz LI, Elson J, Chenarides JO: Preexcilation in athild with syncope. Where is the connetion? J Cardiovasc Electrophysiol 1998;9:892-895.

6. Salte III D, Van Hare GF" Prexcitation secondary to fasciculoventricular pathway in children: a report of three cases. J Candiovascular Elentaphysiol 1999; 10:36-42.

7. Josephson ME. Preexcitation syndromes. In: Josephson ME (ed). Clinical Cardiac Electrophysiology. Techniques and Interpretatons. Philadelphia: Lippinoot Williams \& Witkins, 2002:419-421.

8. Abbotl JA, Scheinman MM, Morady F, eft al: Coexistent Mahain and Kent accessory connections: diagnostic and therapeutic implications. I An Coll Cardiol 1987;10:364-372.

9. Bathassen B: Adenosine triphosphate in candiac arrhytmias: from therapentic to cliagnostic use. $P A C B 2000,25,98+102$

10. Garrat CJ, Griftith $M$, ONunain $S$, et all: Effects of intravenous adenosine on antegrade refractoriness of accessory atroventricular connections. Circulation $1991,84,1962-1968$.

11. Colavita PG, Packer DL, Pressley JC, al: Frequency, diagnosis and clinical characteristics of patients with multiple accessory atrioventricula pathways. An J Cardiol 1987;59:601-606 


\section{Chapter}

\section{The electrocardiogram in patients with}

Fasciculoventricular pathways: a

comparative study with anteroseptal and midseptal accessory pathways

Eduardo Back Sternick ${ }^{*}$

Luz-Maria Rodriguez ${ }^{\dagger}$

Luiz M. Gerken*

Hein JJ Wellens ${ }^{\dagger}$

Biocor Instituto", Nova Lima, Brazil

University Hospitalt, Maastricht, The Netherlands 


\begin{abstract}
Background: Electrophysiologic lindings suggest that fasciculoventricular pathways insert into the septal region. It is also suggested that the surface 12-lead ECG during simus whythm is similar to those of patients with anteroseptal and midseptal bypass tracts.

Objective: To describe the electrocardiographic profile of a cohort of patients with fasciculoventricular pathways and compare it with the ECG of patients with anteroseptal, and midseptal accessory pathways.

Methods and results: Four different observers analyzed the 12-lead ECG of 7 patients with fasciculoventricular pathways (group 1) and the ECG of 20 patients with midseptal A-V accessory pathways (group II), and 20 patienis with anteroseptal $A-W$ accessory pathways (group III). The following variables were analyzed: the ECG frontal plane QRS and delta wave axis, the angle between the QRS and the delta wave axis, QRS width, the $\mathrm{R} / \mathrm{S}$ ratio in lead III, the presence of a negative delta wave in inferior leads, and precordial lead transition to a $R / S>1$.

The ECG of fasciculoventricular pathways shows a variable pattern. It shares some features with an anteroseptal A.P and others with a midseptal AP. A narrower QRS width was the most important variable in distinguishing a fasciculoventricular pathway from a septally located $A-V$ bypass tract $(p<0.0001)$. The angle between QRS and delta wave frontal plane axis could differentiate a fasciculoventricular pathway from an anteroseptal bypass tract $(p<0.0001)$ but not from a midseptal AP.

Conchusion: The sinus rhythm ECG of patients with fasciculoventricular pathways shows similarities with anteroseptal and midseptal accessory pathways, but the QRS complex are usually narrower. Fasciculoventricular pathways with a broad QRS complex can not reliably be differentiated from an anteroseptal or a midseptal bypass tract on the ECG during sinus rhythm.
\end{abstract}




\section{INTRODUCTION}

Fasciculowentricular pathways are rare vartants of pre-excitation taking off from the bundle of His or the bundle branches and inserting into the ventricular septum?. The electrocardiographic recognition of a fasciculoventricular pahway is based upon the finding of a minimal pre-excitation pattem with a normal QRS frontal plane axis with a variable PR interval. ". Although playing no active role in tachycardia circuits, fasciculoventricular pathways are frequently associated with rapidly conducting bypass tracts, and they must be correctly differentiated from an anteroseptal bypass tract, to avoid harm to $\mathrm{A}-\mathrm{V}$ nodal conduction if they are targeted for catheter ablation". There are very few published data on the electrocardiogram of fasciculoventricular pathways ${ }^{3-6}$. We carried out this study to describe the electrocardiographic profile in a cohort of 7 patients with fasciculoventricular pathways and compared their characteristics with the ECG pattern of patients having $A-V$ bypass tracts inserting in the anteroseptal and midseptal region.

\section{METHODS}

\section{Study population}

Fasciculoventricular pathways (group 1): During the last 9 years, 8 patients (2\%) with fasciculoventricular pathways (figure 1) were diagnosed out of 392 patients with manifest ventricular pre-excitation referred to our laboratory for electrophysiologic evaluation. In 3 of the 8 patients $(38 \%)$ the fasciculoventricular pathway could only be diagnosed after catheter ablation of a rapidly conducting bypass tract. One of the 8 patients lacked some electrophysiologic data and was deleted from this study. One of them (case 3 ) had 2 rapidly conducting accessory pathways and had an episode of aborted sudden cardiac death. Six patients were male. Mean age was $29 \pm 16$ (range 13 to 54) years. Four patients were referred because of paroxysmal tachycardia, 2 patients were asymptomatic and were studicd for risk assessment of preexcitation and I patient had palpitations. Two patients (29\%) had paroxysmal $A-V$ nodal reentrant tachycardia and one of them also had non sustained repetitive atrial tachycardia (table 1). Crotp wh artoventricular accessory pathwoys: 40 patients (17 were male) with a single accessory $\mathrm{A}-\mathrm{V}$ pathway with a mean age of $28 \pm 13$ (range 12 to 55 ) years. 20 Patients had a midseptal AP (group II), 13 of them located close to the CS ostium and 7 located at the apical region of the triangle of Koch, and 20 patients with an anteroseptal AP (group TII). We used the method to analyze the ECG from patients with septal bypass tracts described by Rodriguez et al".

None of our patients had additional congenital or acquired cardiac abmormalities which could have affected the QRS morphology. The electrocardiograms were analyzed independently by the four authors. The electrocardiographic data analyzed were: QRS axis and delta wave axis in the frontal plane, the angle between the QRS and the delta wave frontal plane axis, the R/S ratio in llead III, presence of initial negativity in the inferior leads, the $R / S>1$ transition in the precordial leads, PR interval and the QRS width. 
Table 1

Clinical, electrocardiographic and electrophysiologic data of patients with fasciculoventricular pathways

\begin{tabular}{|c|c|c|c|c|c|c|c|c|c|c|c|c|}
\hline Cate & Age & sex & Symptom & EPS & BT Silte & PR & $\begin{array}{c}\text { aRS } \\
\text { width }\end{array}$ & $\begin{array}{l}\text { OR:S } \\
\text { anis }\end{array}$ & $\begin{array}{c}\text { Angle } \\
\text { ans toetia }\end{array}$ & $\mathrm{AH}$ & HV & $W$ \\
\hline 1 & 5月 & M & no: & & & 0.10 & 0.10 & 80 & 20 & 60 & 25 & 320 \\
\hline 2 & 19 & $M$ & palpitation riss & & & 0.09 & 0.12 & 70 & 40 & 80 & 25 & 380 \\
\hline 3 & 19 & $\mathrm{M}$ & tachyourdea & WFW & LLHRMS & 0.12 & 0.12 & 50 & 20 & 75 & 25 & 290 \\
\hline 4 & 21 & $M$ & no & & & 0.11 & 0.12 & 80 & 0 & 80 & 19 & 350 \\
\hline 5 & 54 & $M$ & tachyeardla & AVINET & & 0.09 & 0.09 & 15 & 45 & 55 & 29 & 360 \\
\hline 6 & 43 & $M$ & tachyeardis & WPW & LE & 0.11 & 0.15 & 30 & 30 & 80 & 20 & 320 \\
\hline 7 & 27 & $\mathrm{~F}$ & tachyceardia: & AVRART+AT & & 0.11 & 0.15 & 45 & 45 & 60 & 28 & 250 \\
\hline
\end{tabular}

Abbrewiotions: Angle QRS/Deha axis = difference between the frontal plane axis of QRS complex and delta wave, $A T=$ atrial tachycardia, $A V N R T=A-V$ node reentrant tachycardia, BT= bypass tract. EPS se electrophysiologic study, $L L=$ let lateral $A P, P W=$ Wenckebach point, $Q R S$ width $=$

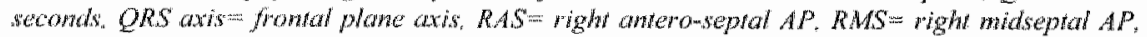
WPW Wolff-Parkinsorn-White syndrome.

\section{Definitions}

Fasciculoventricular pathway: The baseline HV (H-delta) interval during sinus rhythm is $<35 \mathrm{msec}$. During atrial pacing at increasing rates the HV interval does not change. Atrial premature beats cause progressive prolongation of the AH interval without any change in the HV interval and QRS configuration, unless they caused block in the fasciculoventricular pathway resulting in a normal HV interval and narrow QRS complex. Response to adenosine triphosphate suggests a fasciculoventricular pathway when prolongation of the PR interval (AH interval) does not change the degree of pre-excitation or complete AV block occurs after the P wave.

Anteroseptal accessory pathway (AP): bypass tracts lying in close proximity to or immediately anterior to the His bundle. The so-called para-Hissian bypass tracts were included in this group.

Midseptal accessory pathway: bypass tracts located in an area anterior to the coronary sinus and below the His bundle.

Accessory pathway location was established by endocardial activation mapping during sinus rhythm and validated by successful catheter ablation.

Polarity of the delta wave: the first 40 msee vector of the QRS complex.

All patients underwent an electrophysiologic study after discontinuation of antiarrhythmic drug for at least 7 days (no patient was taking amiodarone).

\section{Statistical Analysis}

Comparison between the accessory pathway location groups and their electrocardiographic parameters were anallyzed using the 2-way analysis of variance and the Student Newman-Keuls test. Values are given as mean \pm standard deviation. Statistical significance was assumed for $p$ values of $<0.05$. 


\section{RESULTS}

Group I (Fasciculowentricular pathways): after ablating all associated bypass tracts we found the following ECG and electrophysiologic findings:

Electrocardiogram during sinus thythm: The mean PR interval was $0.10 \pm 0.01$ (range 0.09 to 0.12 ) seconds. Mean QRS complex width was $0.12+0.02$ (range 0.09 to 0.15$)$ seconds. QRS frontal plane axis ranged from 415 to $+80^{\circ}\left(50 \pm 22^{\circ}\right)$. Mean delta wave axis in the frontal plane was $47 \pm 16^{\circ}$ (range 30 to $60^{\circ}$ ), excluding case 8 because the delta wave could not be assessed in all limb leads.

The angle between the QRS and the delta wave frontal plane axis was $24115^{\circ}$. The $R / S$ ratio in lead III was $>1$ in 4 patients, $=1$ in 2 , and $<1$ in 1 patient. The

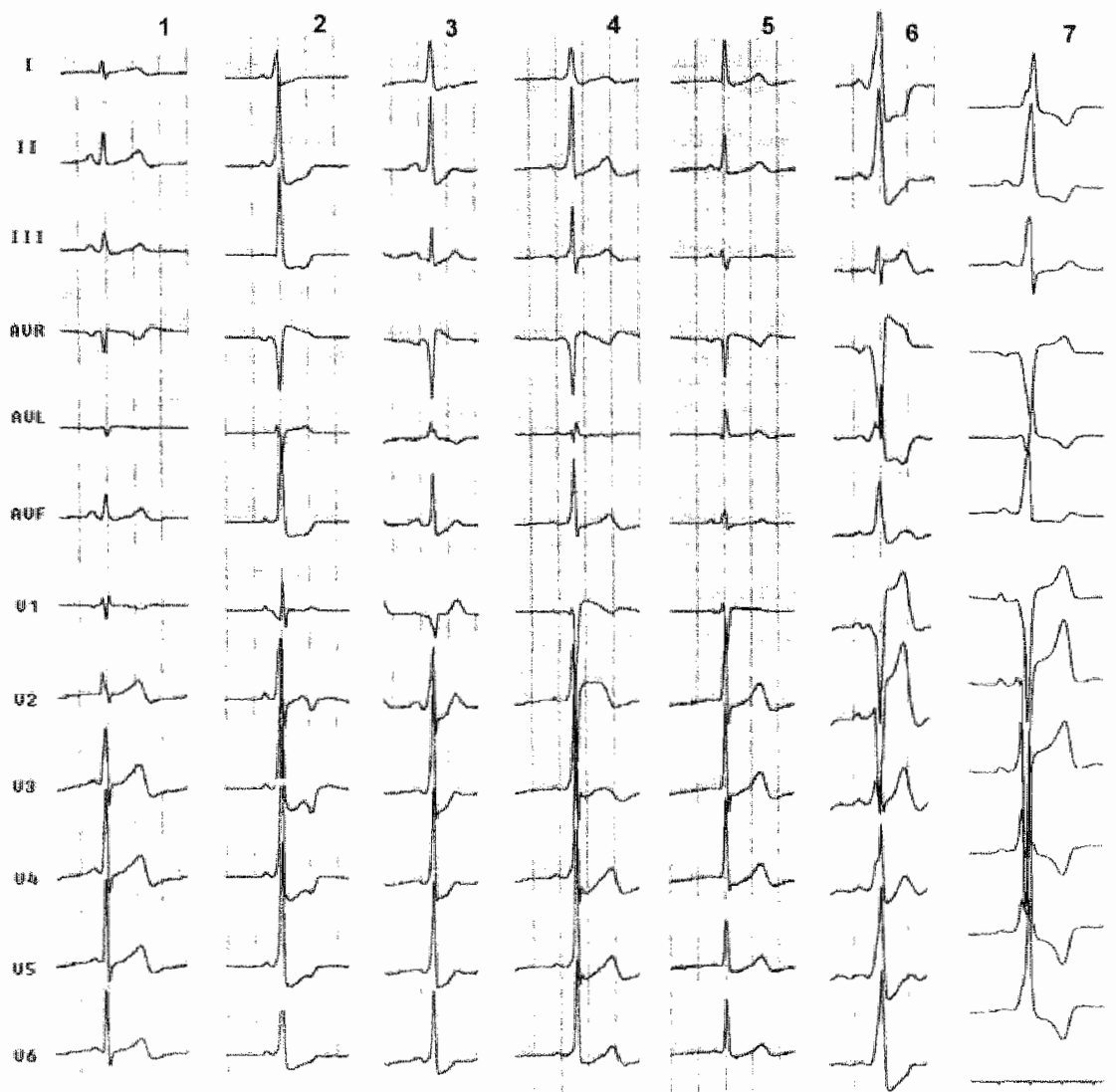

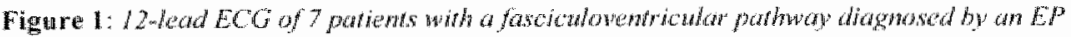

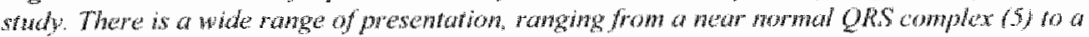
fully preacented $Q R S$ complex 6,7 . 
delta wave was positive in the inferior leads and in $V_{5}$ and $V_{6}$ in all patients (fig. 1). QRS transition $(R / S>1)$ in the precordial leads occurred in lead $V_{2}$ in 5 patients, $V_{3}$ in 1 , and $V_{4}$ in 1 . Patients 6 and 7 , had an ECG pattern indistinguishable from a midseptal bypass tract located at the apical region of the triangle of Koch (figure 2). Patient 1 had a positive delta QRS and the delta wave frontal plane axis was $24 \pm 15^{\circ}$. The $\mathrm{R} / \mathrm{S}$ ratio in lead III was $>1$ in 4 patients, $=1$ in 2 , and $<1$ in 1 patient. The delta wave was positive in the inferior leads and in $V_{5}$ and $V_{6}$ in all patients (figure 1$)$. QRS transition $(R / S>1)$ in the precordial leads occurred in lead $V_{2}$ in 5 patients, $V_{3}$ in 1 , and $V_{4}$ in 1 . Patients wave in lead $V_{1}$, suggestive of a left-sided ventricular insertion, while all the others had a flat or negative delta wave in lead $V_{1}$, consistent with a right ventricular insertion.

Electrophysiologic evaluation: Mean $\mathrm{AH}$ and HV intervals were $62 \pm 10 \mathrm{~ms}$ and $24 \pm 3 \mathrm{msec}$, respectively. During right or left atrial extra-stimulation all patients responded with prolongation of the $\mathrm{AH}$ interval with a fixed HV interval. In 3 of 7 patients $(42 \%)$, a critically timed atrial premature beat blocked the impulse conduction in the fasciculoventricular pathway, causing prolongation of the HV interval and normalization of the QRS complex (figure 3). ECG during AVNRT showed a wide QRS tachycardia due to bystander anterograde conduction over a fasciculowentricular pathway in case 7 (figure 4).

All patients received intravenous adenosine as a bolus and responded with prolongation of the PR interval (AH interval) without changing the degree of preexcitation (and the HV interval). During adenosine conducted atrial beats always showed pre-excitation (figure 5 ).

\section{Group II (Groups of patients with accessory atrio-ventricular pathways)}

1. QRS axis in the fromtal plane.

Midseptal AP: -15 to $+60^{\circ}$ (mean $32 \pm 22^{\circ}$ ); anteroseptal AP: 0 to $+75^{\circ}$ (mean 45 $\left.+25^{0}\right)$.

2. Delta wave axis in the frontal plane

Midseptal and anteroseptal AP had an intermediate delta wave axis: 0 to $60^{\circ}$ $\left(\right.$ mean $\left.23 \pm 18^{\circ}\right), 0$ to $60^{\circ}$ (mean $43 \pm 18^{\circ}$ ), respectively.

3. The angle between the QRS and the delta wave axis: midseptal: 10 to $40^{\circ}$ (mean $23 \pm 8^{0}$ ), anteroseptall accessory pathway: 0 to $15^{\circ}$ (mean $\left.4 \pm 8^{0}\right)(\mathrm{p}<$ 0.0001 , as compared with group I patients).

4. ORS with: the mean QRS width in the 40 patients with accessory A-V pathways was $0.14+0.01$ ( $\mathrm{p}<0.0001$, as compared with the group I patients).

S. The $R / S$ ratio in lead III:

Seven of the 20 patients with midseptal $A P$ had an $R / S$ ratio equal to 1 in lead $I I I$ and the $R / S$ ratio was $<1$ in the remaining 13 patients. All 7 patients with midseptal accessory pathways wilh an $R / S=1$ had their AP located in the apical part of the triangle of Koch, just below the bundle of His. In all patients with anteroseptal $A \mathrm{P}$ the $\mathrm{R} / \mathrm{S}$ ratio in lead 111 was $>1$.

6. Delta wave negativity in inferior leads: 


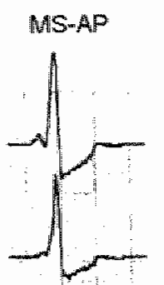

FWP.6

MS-AP

Fip.
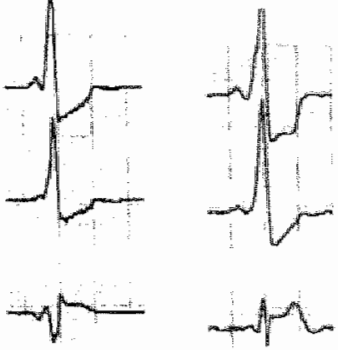

1.1.<smiles>CCCC(C)CC(C)C</smiles>

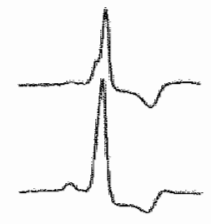

III
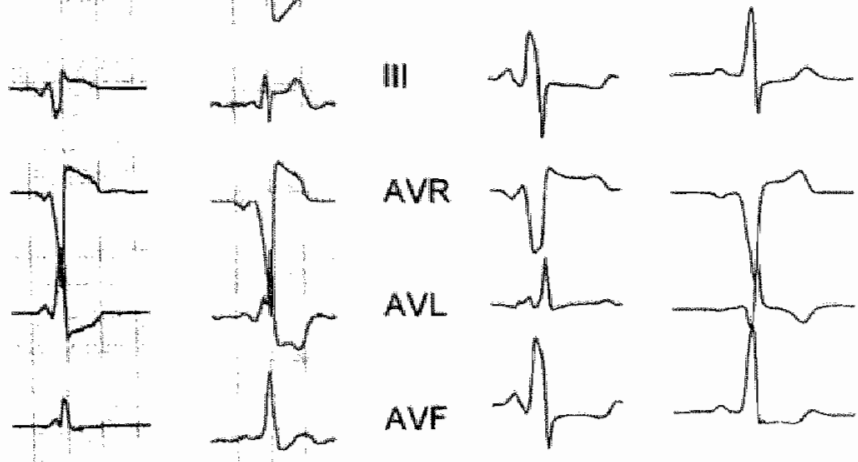

AVR

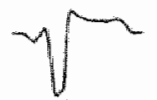

AVL<smiles>CCC(C)C</smiles>

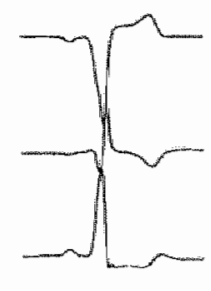

AVF
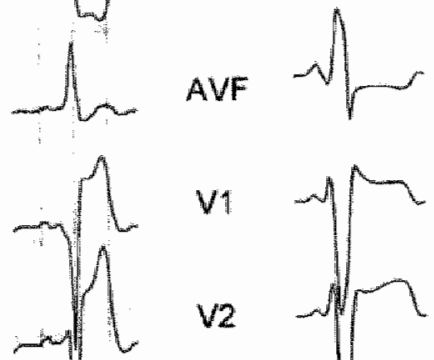

$v_{1}$
v2
v3
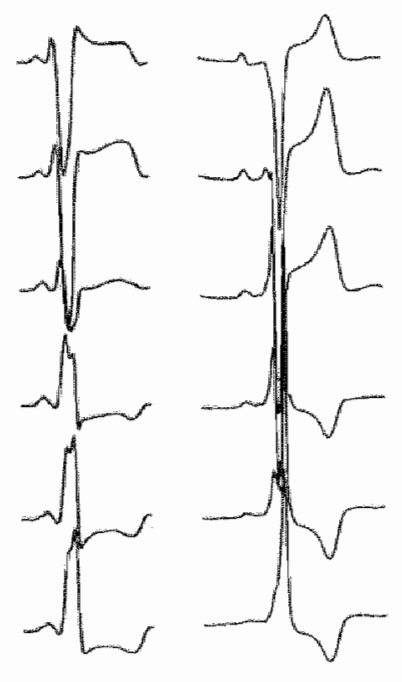

$Q$

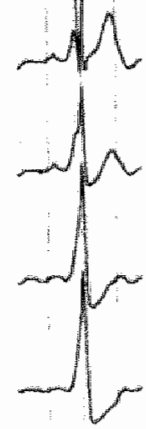

V4

V5

16

Figure 2: ECGs of parients 6 and 7 with a faseiculowenricular pathenys (FVP) showing a broad RS complex. They are displayed for comparison with ECO of 2 Grow II pasiends with midseptal bypass tracts $(M S-A P)$ 


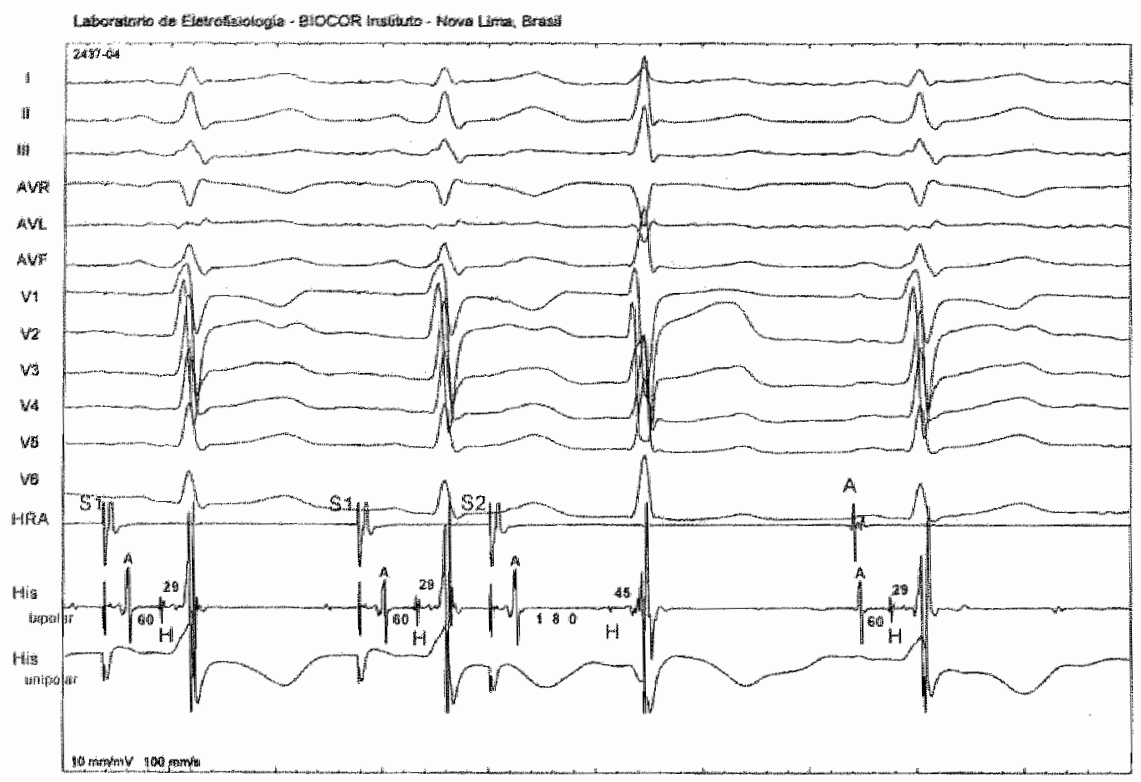

Figure 3: Case 5-A critically thed arial prematwe bea blacked in the fasciculoventricular

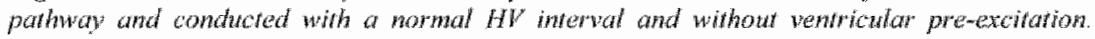
Show are recordings from surface leads, and intracandiac electrograms from the high laterat

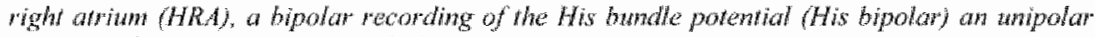

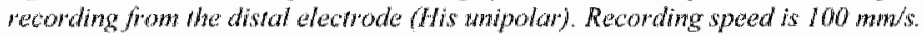

Midseptal accessory pathways showed positive delta waves in lead III in 12 of the 20 patients and a negative della wave in lead III in 8 of 20 . All 20 patients with anteroseptal AP showed positive delta waves in the inferior leads.

7. Precordial lead tramsition to an $R / S$ ratio $>I$

Midseptal AP: 5 patients showed R/S $>1$ in $V_{2}, 11$ in $V_{3}$, and 4 in $V_{4}$. Anteroseptal AP showed a shift to an R/S ratio $>1$ mainly in $V_{4}(16 / 20$ patients), and less often in $V_{s}(4$ patients)

\section{DISCUSSION}

The septal region is a complex anatomic region harboring not only the fasciculoventricular pathways but approximately $30 \%$ of all accessory atrioventricular pathways. As a consequence, it is expected that the ECO presentation of the fasciculloventricular pathway may share some characteristics with septal atrioventricular pathways. Applying previously published algorithms in the diferential diagnosis of septal accessory pathways in patients with fasciculoventricular pathways they will be categorized as anteroseptal or midseptal bypass tracts ${ }^{\text {? }}$. Patients with fasciculoventricular pathways show a wariable PR interval. Some authors describe a nomal or short PR interval. Callagher et al reported 6 patients with fasciculoventricular pathways with a PR interval $<0.12$ seconds and electrophysiologic evidence of enhanced $A-V$ nodal 


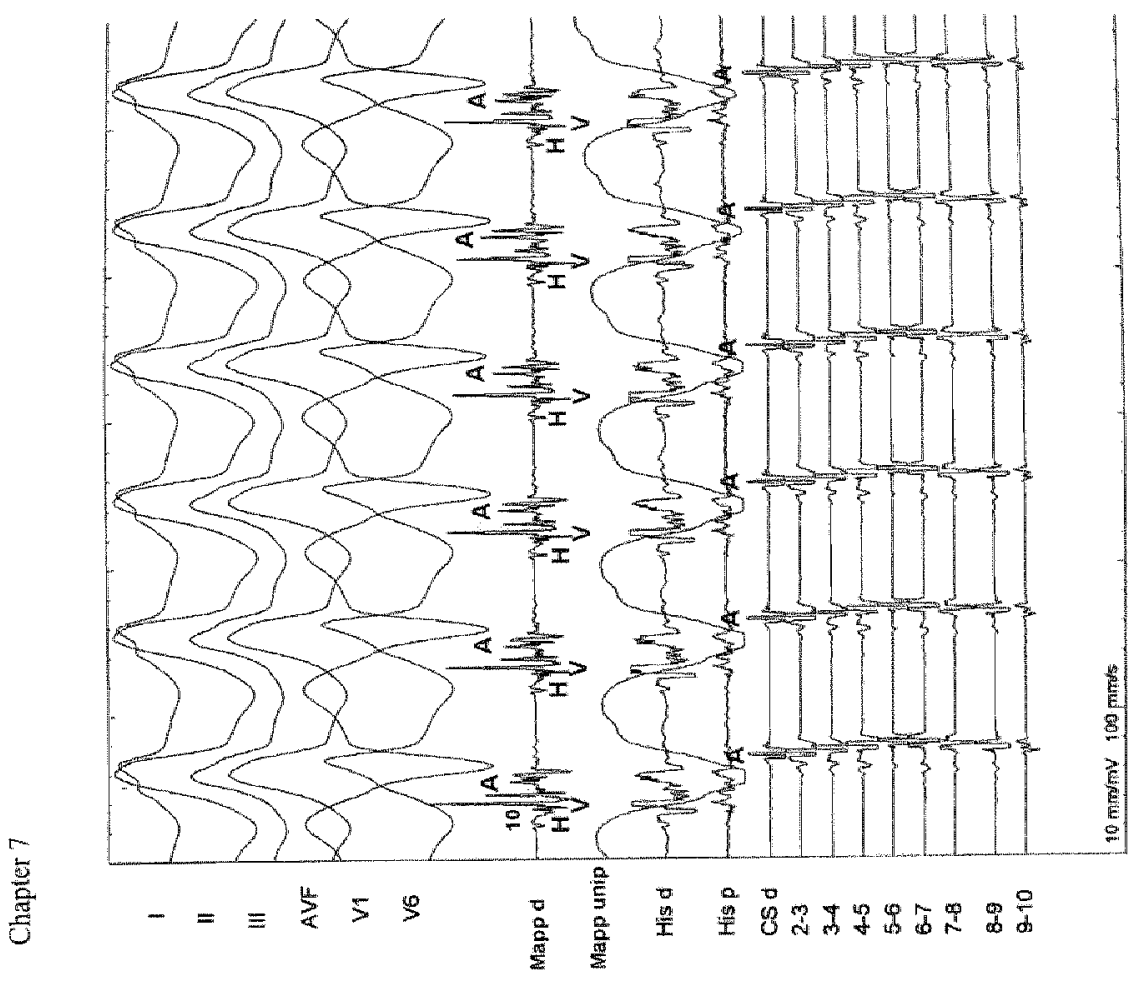

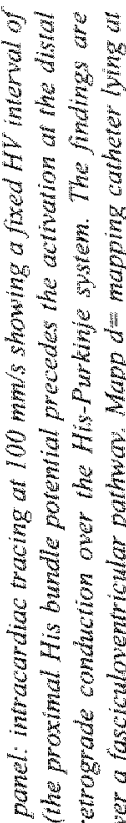

2

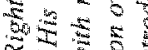
2. $5 \&$

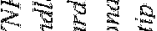

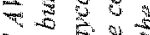
\& के 250 \%.

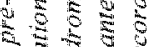
20,5 与

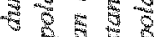

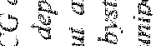
प w) 7 है

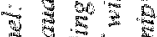
5

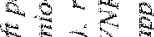
और $\therefore \therefore$ 羟 $y$ \& $0 \leqslant 0$ ?

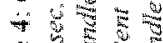
然语 봉요

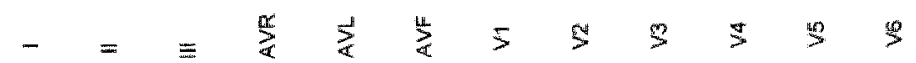


conduction. In our series the mean PR interval was $0.10 \pm 0.01$ (range 0.09 to 0.12 ) seconds, but no patient had enhanced $A-V$ nodal conduction. Previous studies ${ }^{50}$ concentrated on the QRS configuration and delta wave morphology in lead $V_{1}$ which in our cohort showed a wide variability. According to our findings fasciculoventricullar pathways have overlapping electrocardiographic features with both anteroseptal and midseptal accessory bypass tracts. The mean QRS and delta wave frontal plane axis was normal in all 3 groups.

\section{Electrocardiographic similarities with anteroseptal accessory pathways}

The $\mathrm{R} / \mathrm{S}$ ratio in lead III was $>1$ in 4 of the 7 patients $(57 \%)$ with fasciculoventricular pathways and in $100 \%$ of the patients with an anteroseptal AP $(p=n s)$. In contrast, patients with midseptal accessory pathways never showed an $\mathrm{R} / \mathrm{S}$ ratio $>1$.

Patients with fasciculoventricular pathways and patients with anteroseptal bypass tracts never showed a negative delta wave in inferior $\|$ eads, in contrast with $25 \%$ of the patients with a midseptal accessory pathway.

\section{Electrocardiographic similarities with midseptal accessory pathways}

Patients with fasciculoventricular pathways and patients with a midseptal bypass tract had a similar angle between the QRS and the delta wave axis in the frontal plane $\left(24 \pm 15^{\circ}\right.$ and $23 \pm 8^{\circ}$, respectively) as compared with the angle of $4 \pm 8^{\circ}$ in patients witt an anteroseptal bypass tract $(p<0,0001)$.

\section{Electrocardiographic dissimilarities between fasciculoventricular pathways, midseptal, and anteroseptal accessory pathways}

The transition to an $R / S$ ratio $>1$ in the precordial leads occurred mainly in $V_{2}$ in patients with fasciculoventricular pathways, $V_{3}$ in midseptal pathways and $V_{4}$ in anteroseptal bypass tracts.

The major feature differentiating fasciculoventricular pathways from a septal bypass tract was the QRS width: $0.12 \pm 0.02 \mathrm{~ms}$ in the former and $0.14 \pm 0.008$ and $0.14 \pm 0.01 \mathrm{~ms}$ (midseptal and anteroseptal bypass tracts, respectively) ( $\mathrm{p}<$ $0.0001)$.

The $\mathbb{E C G}$ during sinus rhythm in patients with fasciculowentricular pathways will show a short PR interval, usually with a minimal pre-excitation pattern with a QRS width of 0.12 seconds, a normal frontal plane QRS and delta wave axis, a short angle between the QRS and the delta wave axis and a precordial R/S $>\mathbb{1}$ transition most likely in $V_{2}$. It is worth mentioning that no patient with an anteroseptal or a midseptal bypass tract had minimal preexcitation.

Patients with left-sided bypass tracts may show minimal pre-excitation. According to Bogun et al ${ }^{10}$ an $r s R$ " is usually found in those patients in lead V 6 , in contrast with a $R$ pattern found in our 8 patients with fasciculoventricular pathways. Mahaim fibers of the atriofascicular variety usually show minimal prexcitation, but an ${ }^{S} S$ pattern can be found in lead $1 \mathrm{II}^{\mathrm{h}}$, while fasciculoventricular pathways usually show a $\mathrm{R} / \mathrm{S}$ ratio $>1$ in lead III.

\section{Clinical implications}

Fasciculoventricular pathways are responsible for a variable pre-excitation pattern on surface ECG, as shown in this study. The ECG is similar to ECG of patients with antero-septal and midseptal accessory atrioventricular pathways. A negative test with intravenous adenosine calls for an electrophysiologic study for a correct 

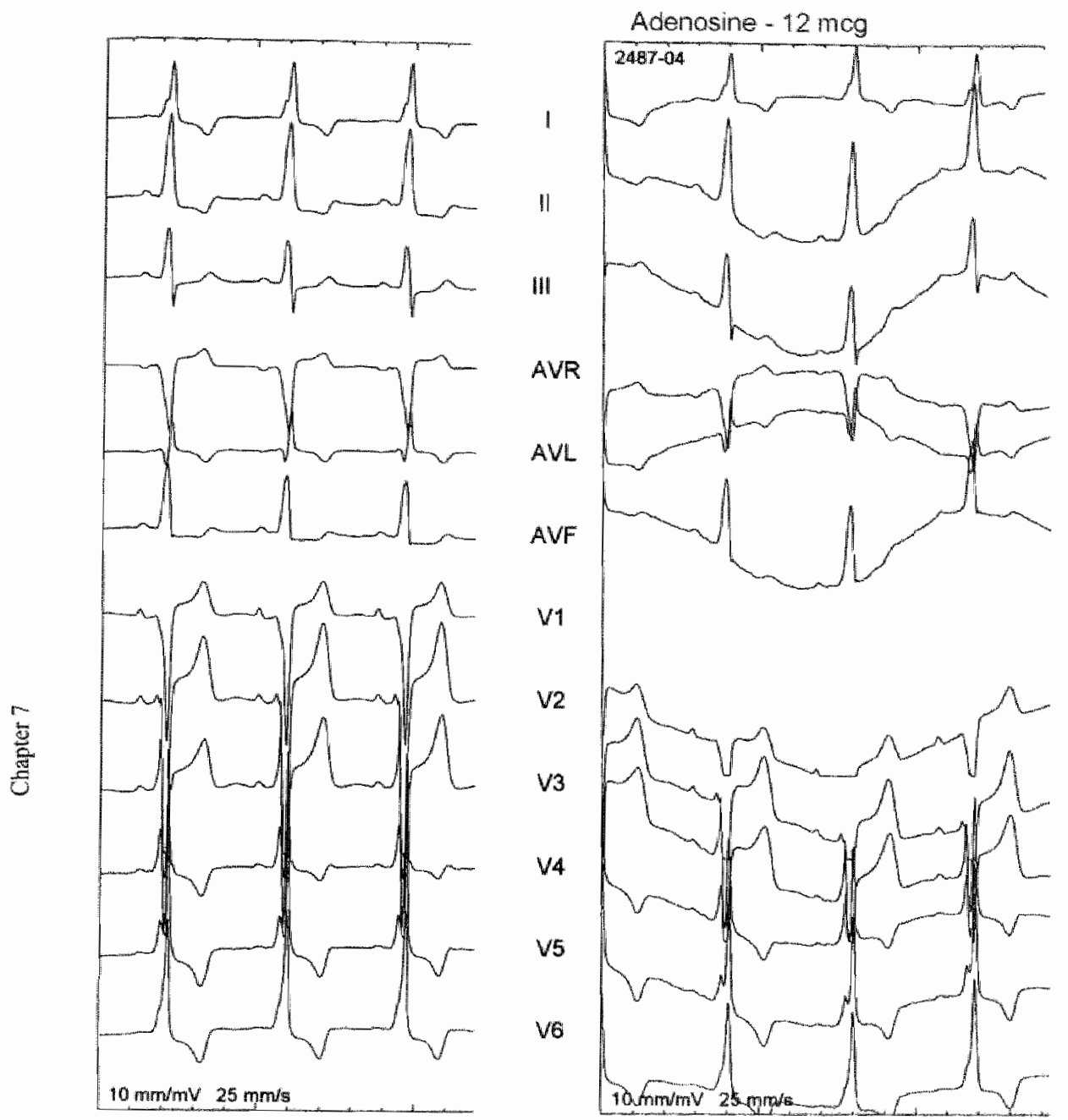

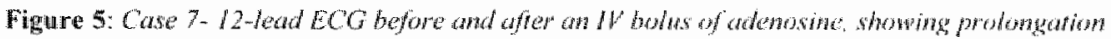

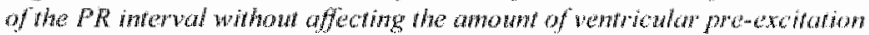

diagnosis. It has been shown that fasciculoventricular pathways may be associated with other arrhythmogenic substrates like dual AV nodal pathways or rapidly conducting $\mathrm{AV}$ bypass tracts, and be involved as bystanders during supraventricular tachycardia, causing ECG patterns of difficult interpretation as shown in figrure 4.

\section{Limitations of the study}

The main limitation to our study relates to the fact that the fasciculowentricular pathways were not ablated, and we assume that they have a ventricular septum connection based upon the finding of an carliest ventricular activation close the bundle of His. The number of patients with fasciculoventricular pathways is too small to come to a definite conclusion. 
$\frac{1}{3}$

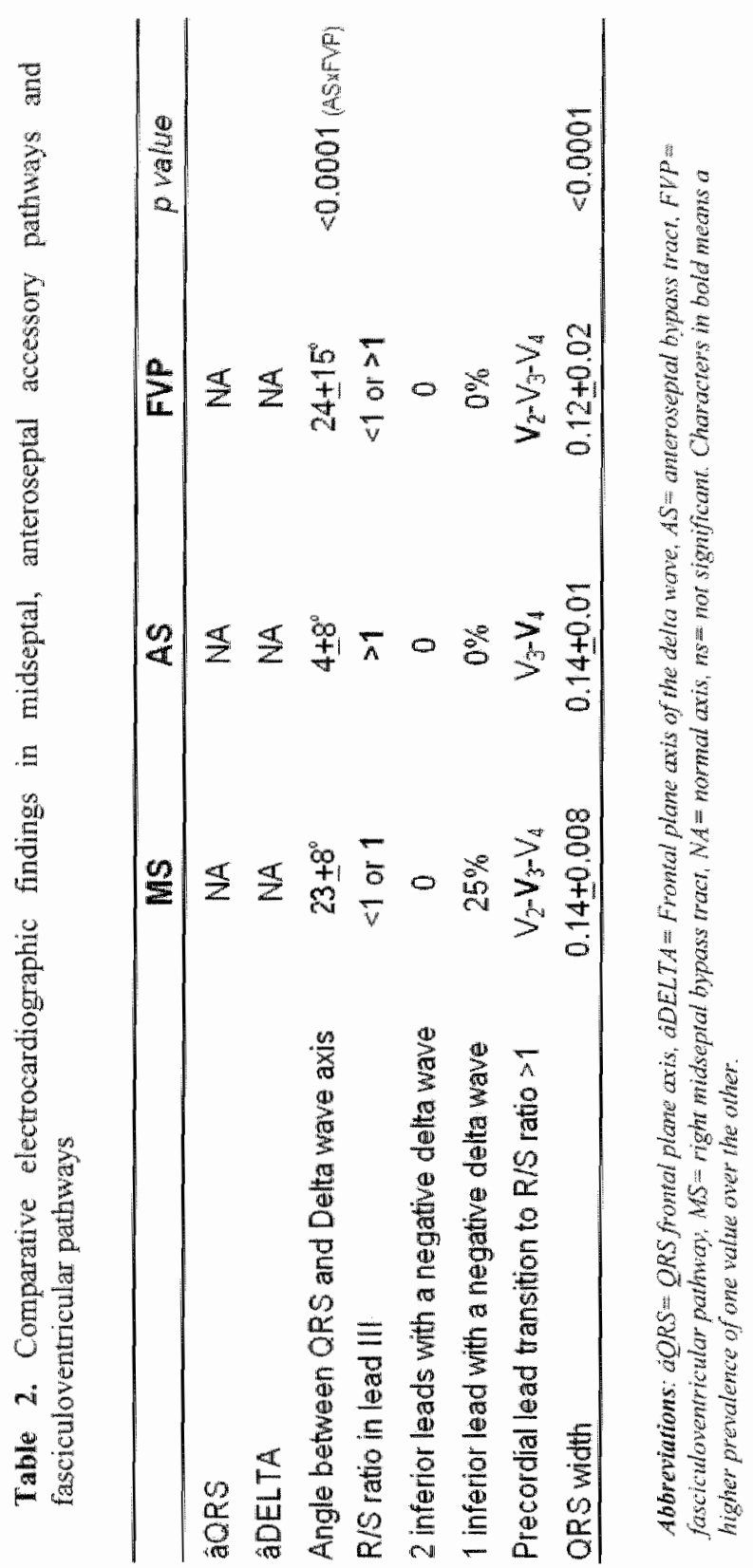




\section{CONCLUSION}

The ECG of patients with fasciculoventricular pathways shows similarities with anteroseptal accessory pathways and midseptal bypass tracts located at the apex of the triangle of Koch, but the QRS complex are usually narower. Fasciculoventricular pathways with a large QRS complex can not reliably be differentiated from an anteroseptal or a midseptal bypass tract by the surface ECG. The definite diagnosis requires an intracardiac study observing the presence or absence of changes in the QRS complex during single test atrial stimulation and atrial pacing at increasing rates. This should always be done when ablation of septally located accessory pathway is considered.

\section{References}

1. Josephson ME. Preexcitation syndromes. In: Josephson ME (ed). Clinical Cardiac Electroplysiology. Techniques and Interpretations. Phitadelphia: Lippincot Williams \& Wilkins, 2002:419-421.

2. Lev M, Fox SM, Bharati S, Greentield IC, Rosen KM, Pick A: Mathaim and James fibers as a basis for a unique wariety of ventricular preexcitation. An J Cardiol 1975;36:880-8.

3. Gallagher J, Snith WM, Kasell JH, Benson DW, Sterba R, Gram AO: Role of Mahaim tibers in cardiac arrbyommas in man. Circulation 1981;64:176-189.

4. Stermick EB, Gerken LM, Vrandecic MO, Wellens HJ: Fasciculoventricular pathways: clinicnl and electrophysiologio characteristics of a variant of preexcitalion. I Cardiovase Flactrophysiol $2003 ; 14: 1057-1063$.

5. Myaguchi K, Tsuzuki J, Yokota M, Hayashi H: Characteristic findings on the standard 12-lead DOG in patients with the fasciculoventricular Mahaim tiber. I Electocardion 1992;25:253-61.

6. Ito $M$, Onodera $S$, Noshiro H, Odakura H, Yasuda S, Nagashima $M$, Shinoda $S$, Suzuki Ht: Encet of class IA antiarrhythmic agents on fasciculowentricular fibers. J Electrocardiol 1990;23:32:3-9.

7. Rodriguez LM, Smeets IL, de Chillon C, Metzger I, Schlapler I, Perm O, Weide A. Westlens HJJ: The 12-lead electrocardiogram in midseptal, anteroseptal, posteroseptal and right free watl atecssory pathways. Am \& Cardiol 1993;72:1274-1280.

8. Finzpatrick AP, Gonzales RP. Lesh MD, Modin GW, Lee RJ, Scheinman MM: New allgorithm for the localization of accessory atrioventricular connections using a baseline electrocardiogram. I Am Coll Cardiol 1994;23:107-116.

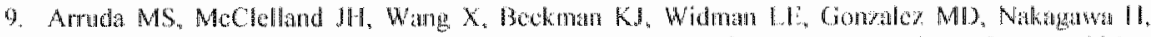
Lazara R, Jackman WM: Development and waldidtion of an ICCG algorithm for identifying aceessory pallway ablation ste in the Wolr-parkinsm-White syndrome. I Condiownc Electrophysiol 1998:9:2-12.

10. Bogun F, Katusche D, Li YG. Auh-Eisernic S, Groncheld G. Hohnloser SH: Septal Q waves in

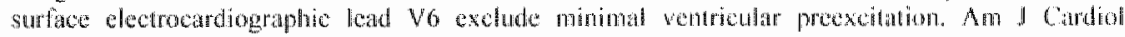
$1099,84: 101-104$.

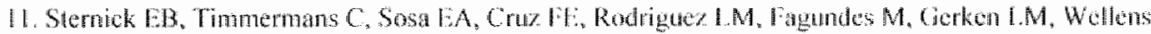

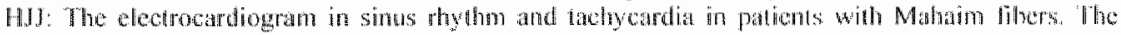
importance of an " 15 " paittern in lead 111 . A Am Coll Cardiol $2004,44: 1626.1635$. 


\section{Chapter 8}

\section{Mahaim fiber: an atriofascicular or a long atrioventricular pathway?}

Eduardo Back Sternick*

Carl Timmermans ${ }^{\dagger}$

Luz-Maria Rodriguez ${ }^{\dagger}$

Hein JJ Wellens ${ }^{\dagger}$

Biocor Instituto", Nova Lima, Brazil

University Hospitalt, Maastricht, The Netherlands

Accepted for publication in Heart Rhythm 


\begin{abstract}
Introduction: There is debate concerning the distal insertion of Mahaim fibers. Some findings favor an atriofascicular fiber connected with the distal right bundle branch while others favor a long atrioventricular structure inserting into the myocardium.

Case report: A patient is reported having a decrementally conducting accessory pathway. Proximal and distal Mahaim potentials were recorded during sinus rhythm, atrial pacing and antidromic tachycardia. Both proximal and distal $M$ potentials always preceded the QRS complex during sinus rhythm and antidromic tachycardia earlier than the right bundle branch potential. During tachycardia the distal $M$ potential was recorded $6 \mathrm{msec}$ before the retrograde right bundle potential. Other arguments consistent with an atrioventricular connection were a change in the QRS configuration during tachycardia after the first radiofrequency pulse at the site of the distal $M$ potential, and absence of right bundle branch block after successful ablation. Conduction through the proximal part of the Mahaim fiber was unaltered after ablation, as assessed by recording the proximal $M$ potential.

Conciusion: Electrophysiologic evidence is presented suggesting a long atrioventricular accessory pathway inserting close to the distal right bundle branch rather than an atriofascicular connection in this patient with a Mahaim fiber.
\end{abstract}




\section{INTTRODUCTION}

For more than 30 years the proximal part of a Mahaim fiber was believed to take off from the $A-V$ node ${ }^{1-3}$. These $A-V$ node-like structures are accessory pathways with long and decremental conduction and are now usually classified as a short atrioventricular pathway, when ventricular activation mapping during sinus thythm shows early activation at the tricuspid annulus or as an atriofascicular pathway when ventricular activation is found at the lower right ventricle free wall ${ }^{4-5}$. However, some studies raise the issue of insertion of the distal end of those long decremental pathways in the right bundle branch ${ }^{6}$. We present a case with a long Mahaim fiber which was carefully mapped both at is proximal and distall end. Successful radiofrequency catheter ablation aimed at the distal insertion did not cause right bundle branch block.

\section{CASE REPORT}

A young female aged 22 was admitted because of frequent episodes of a left bundle branch block-like tachycardia since the age of 16 years. She had a history of many hospital admissions for acute treatment of her tachycardias which were always terminated with A-V node blocking agents such as verapamil or adenosine. Physical examination, the 12-lead ECG during sinus thythm and a transthoracic echocardiogram showed no abnormalities. The ECG during regular tachycardia showed a QRS complex (width of $130 \mathrm{msec}$ ) with a LBBB-like pattern and a superior frontal plane axis $\left(-15^{\circ}\right)$. The tachycardia was regular with a $1: 1 \mathrm{~A}: \mathrm{V}$ relationship. There was an " $R$ " wave in lead $I$, and an " $\mathrm{S}$ " in VI without notches in the fast downstroke. The R/S ratio became $>1$ in lead VS (figure 1).

Electrophysiologic procedure: The patient underwent the EP study in the fasting non sedated state. Catheters (USCI, Billerica, MA, USA) were positioned in the coronary simus, the His-bundle region, and the high right atrimm. During sinus rhythm the basic intervals (figure 2) were normal $(P A=15, A H=60, R B-V=28$, $H V=40$ msec). During atrial pacing at increasing rates the classic "Mahaim pattern" emerged. During ventricular pacing there was retrograde conduction over the $A-V$ node His-Purkinje systen, and realizing that we were facing a Mahaim fiber we rearranged the catheters as follows: quadripolar catheters were left in the high right atrium, coronary sinus and right ventricular apex. The decapolat catheter was positioned over the His bundle and right bundle area. Two 8-F ablation catheters were used for mapping the proximal $\left(M_{p r o s}\right)$ and distal $\left(M_{\text {dist }}\right)$ accessory pathway potentials at the tricuspid annulus and at the antero-apical area of the right ventricle, respectively. Programmed electrical stimulation was done by using stimuli of 0,5 milliseconds duration at twice diastolic threshold. A 12-lead ECG was stored simultaneously with intracavitary signals on a digital polygraph (EPTRACER, CardioTek BV, Maastricht, The Netherlands). Local electrograms were filtered at $50-500 \mathrm{~Hz}$ and amplified at a gain of $10 \mathrm{~mm} / \mathrm{mV}$. We found a small M potential of low amplitude at the lateral aspect of the tricuspid annulus. The $A$ $M_{\text {pros }}$ interval was $75 \mathrm{msec}$ and the $\mathrm{M}-\mathrm{V}$ interval was $30 \mathrm{msec}$. We attempted to delineate the course of the Mahaim fiber by mapping both the proximal and the distal insertion during simus rhythm. Mapping in the apical region of the right ventricular free wall we found a huge spike-like (high frequency) potential a short distance from the QRS complex $\left(\mathrm{M}_{\text {distal }}-\mathrm{V}=8 \mathrm{msec}\right)$. Both proximal and the distal potentials were simultaneously reconded (figure 2 ). 


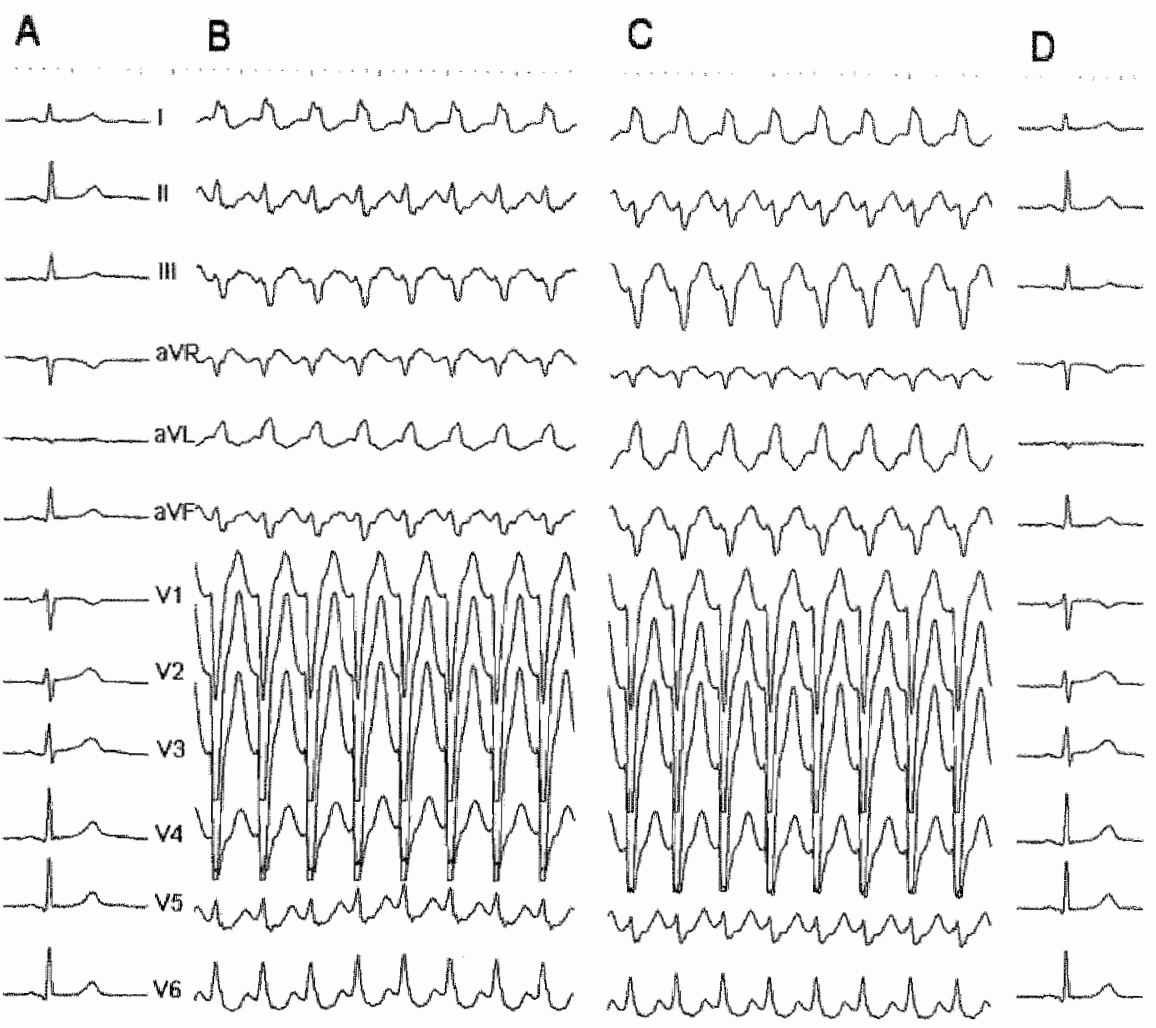

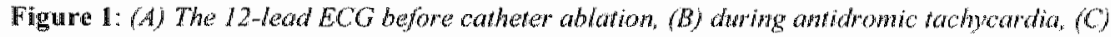
dumg antidromic tachycardia after the first episode of radiofiequency crarent, and (D) aftom suctessyl carherer ablation, without RBBB. Paper speed: $25 \mathrm{nmw} / \mathrm{sec}$.

Induction of tachycardia was possible during atrial and ventricular pacing. Latte atrial premature stimul anticipate wentricular activation during preexcited tachycardia, proving that the accessory pathway was used in the tachycardia circuit. Earliest ventricular activation during tachycardia was recorded at the site where we recorded the distal $M$ potential $\left(M_{d}-V=10 \mathrm{msec}\right.$ and $V$-delta $=-4 \mathrm{msec}$ ) (figure 3 ). Both proximal and distal $M$ potentials always as well as the right bundle branch potentialpreceded the QRS complex during sinus rhythm and antidromic tachycardia but not the His bundle branch potential. During tachycardia the anterograde distal $M$ potential was recorded $6 \mathrm{msec}$ before the retrograde right bundle potential. We decided to ablate the accessory pathway at this distal site during sinus thythm to improve stability of the catheter. However, during current application (15 seconds) we recorded an irregular non sustained thythm. Tachycardia could still be induced after current delivery, but with a slightly different QRS configuration (figure 1). We then applied radiofrequency current at the same site for almost 2 minutes. Thereafter programmed stimulation could no longer induce a tachycardia. Preexcitation was also abolished during atrial stimulation but we were able to record the proximal Mahaim potental with the same timing as before. During atrial pacing with a cycle length of $360 \mathrm{msec}$, Wenckebach block at the $A-V$ node level was achieved but the Mahaim liber was 


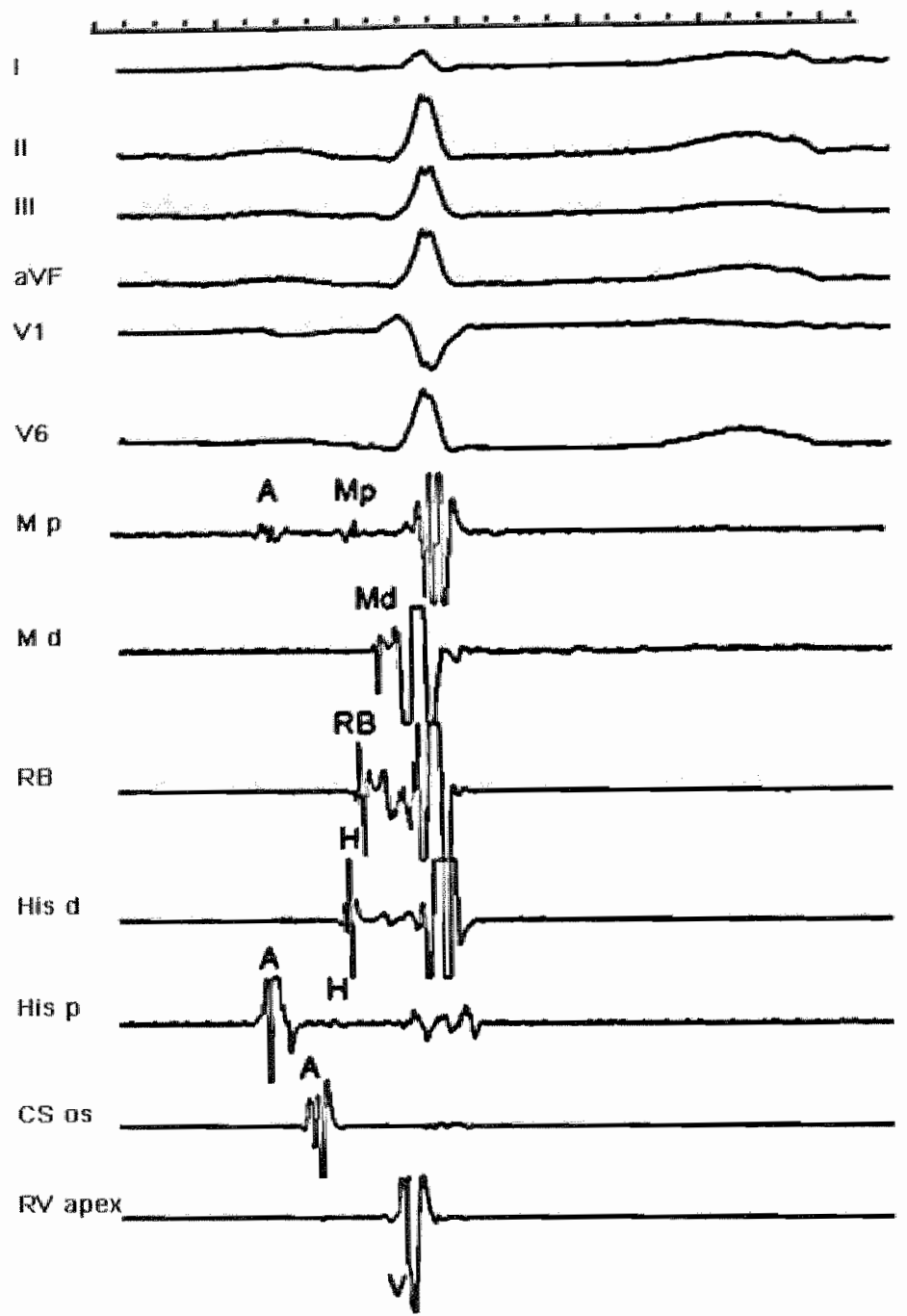

Abbreviations: A-atrial, $\mathrm{Mp} / \mathrm{Md}=$ proximal and distal Mahaim potentials, $\mathrm{H} / \mathrm{RB}=\mathrm{His}$ bundle and righ bundle potentials, $V=$ ventricle

Figure 2: Sinus rhythm. There is no ventricular preexcitation. The $A-H$ interval is shorter than A-Mp interval. The His bundle is depolarized before the right bundle. Paper speed: $200 \mathrm{~mm} / \mathrm{sec}$.

still able to conduct the stimuli through its proximal part, but with complete conduction block at its distal portion (figure 4).

\section{DISCUSSION}

The issue of the correct understanding and delineation of the course and insertion of the so called Mahaim fibers is still open. We know now, that in spite of former 


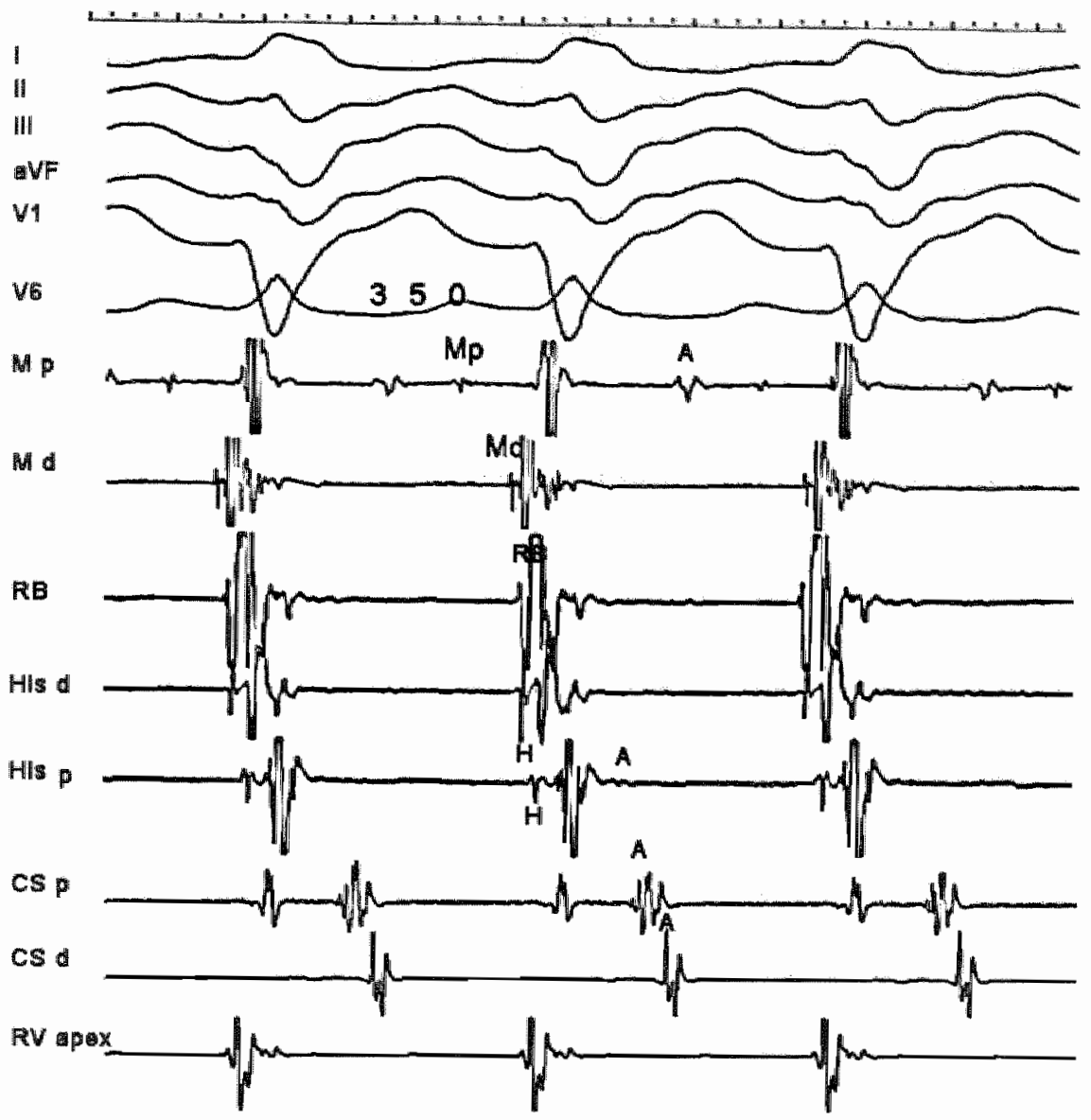

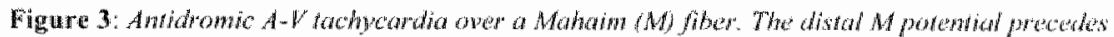

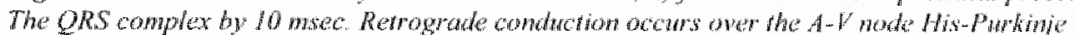

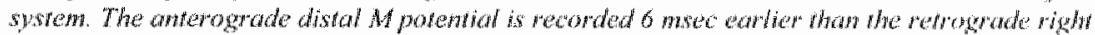

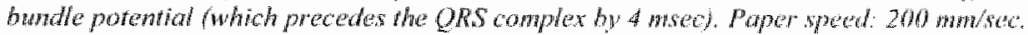

opinions, that Mahaim fibers are usually not connected to the A-V node and HisPurkinje system ${ }^{7.8}$.

Many authors still believe that the distal insertion of the Mahaim fiber is attached to the normal conduction system particularly the right bundle branch, the atriofascicular concept. However, the findings in this case are consistent with a direct myocardial insertion of the Mahaim fiber. The disappearance of the distall M potential after ablation with preservation of the right bundle potential, the absence of right bundle branch block in spite of 2 minutes of radiofrequency current application at that site, as well as the modification of the QRS complexes during tachycardia after the first radiofrequency pulse (figure 1), suggest distal myocardial arborization of this pathway. The occurrence of an irregular antomatic rhythm 
(Mahaim automaticiry) during application of radiofrequency current and achievernent of successful ablation also validate the aforementioned potential as a distal Mahaim potential, and not a right bundle branch potential. In our patient the accessory $\mathrm{A}-\mathrm{V}$ node-like structure length

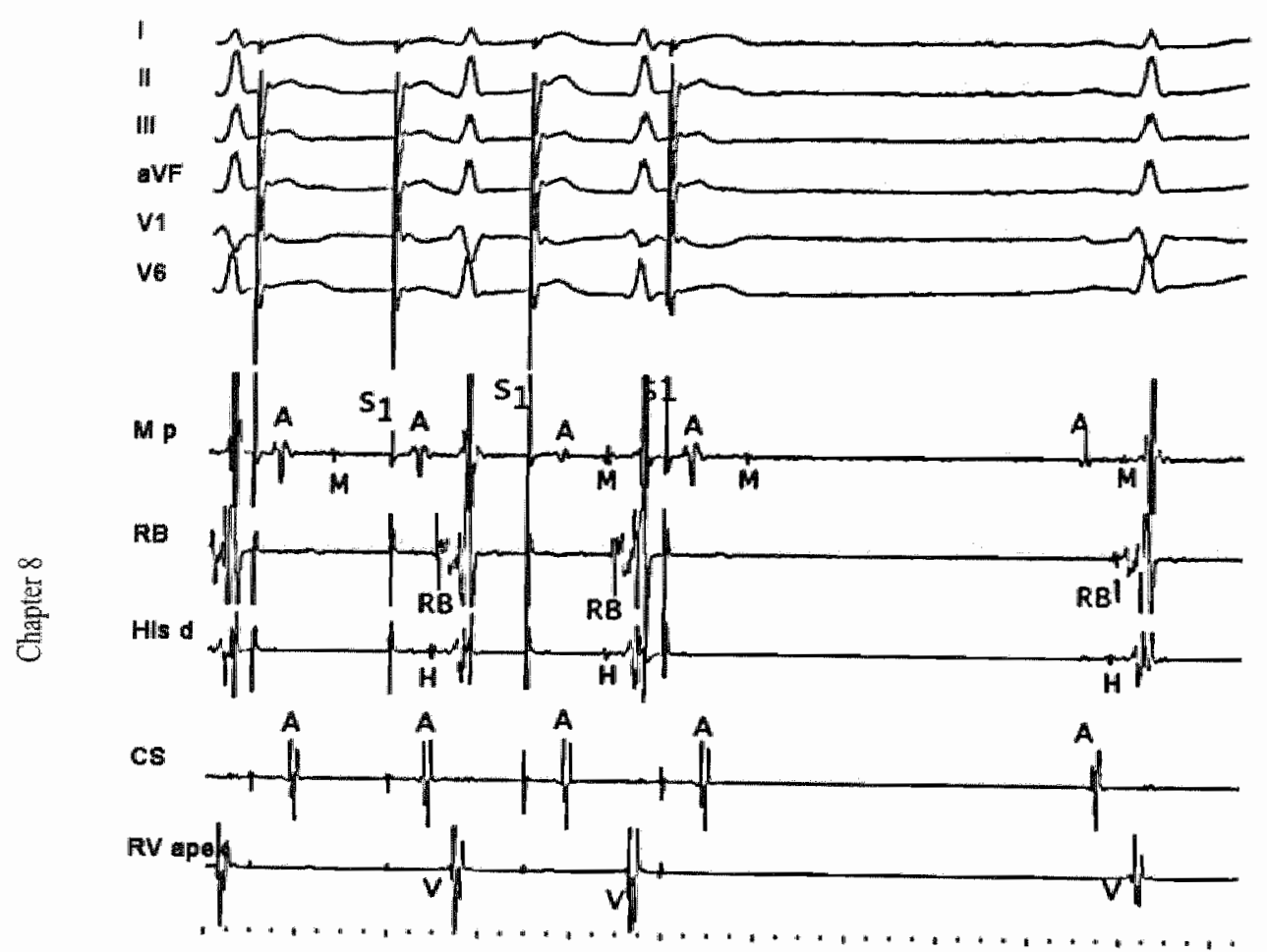

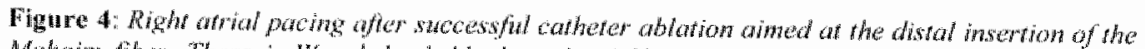

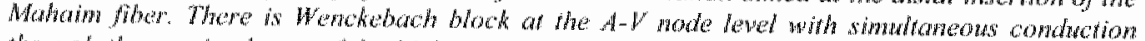

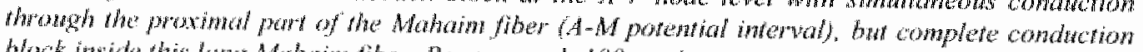

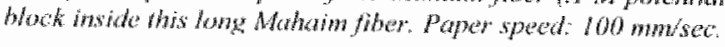

could be radiographically determined by assessing the distance from the proximal to the distal potential as around $5 \mathrm{~cm}$. Other authors have estimated its length from 2 to $5 \mathrm{~cm}$ " Noncontact mapping during antidromic tachycardia in one patient with a posterolateral Mahaim ${ }^{2}$ showed break out of conduction as far as at the apex, validating old data from intraoperative epicardial mapping 4 . The major electrophysiologic argument used in support of the atriofascicular concept is the timing of the spike-like potential of the distal right bundle branch, which is usually recorded at the onset of the QRS complex during preexcited tachycardia (V-RB interval showing a range from 0 to $20 \mathrm{msec}$ ). In case of right bundle branch block this interval increases because retrograde conduction is shifted to the 
left bundle branch. However, recent studies using noncontact mapping system show that right wentricular depolarization starts in normal individuals usually at the right wentricle free wall $-9,8+5,5(-410-15)$ msec before onset of the surface $\mathrm{QRS}^{13}$. As we have shown in this case the distal M potential was recorded 6 msec ahead of the right bundle potential and $10 \mathrm{msec}$ before the preexcited QRS complex, which is consistent with early activation of the right ventricle from a decremental conduction pathway at a small distance from the distal right bundle branch. According to Rudy ${ }^{1 / 4}$ electrical impulse propagates through the ventricle with a conduction velocity of $60 \mathrm{~cm} / \mathrm{second}$. We can estimate the distance from the distal Mahaim potential from the site where the distal right bundle branch potential was recorded to be around $3,6 \mathrm{~mm}$. Other authors differentiate atriofascicular from long atrioventricular decremental pathways by looking at QRS duration and to $\mathrm{V}$-His (and V-RB) interval during preexcited tachycardia. Our observation suggests that the distance between the myocardial insertion of the Mahaim fiber and the right bundle branch is the key factor for those differences. The absence of a more pronounced sturring at the onset of the QRS during antidromic tachycardia also favors a small distance between the distal accessory pathway and the distal Purkinje fibers in this case.

\section{Limitations of the study}

In spite of the electrophysiologic evidence suggesting that this fiber inserted directly over the right ventricular myocardium we can not rule out arborization into the distal Purkinje system rather than the myocardium. Likewise the absence of RBBB does not exclude ablation of some distal Purkinje fibers. A strong argument in favor of a long atrioventricular pathway could have been made if ransjent block at the right fascicle either caused by bump napping or cryonapping did not interrupt antidromic tachycardia, but that was not done in this case.

\section{References}

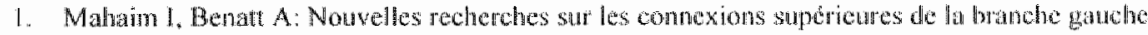
dhi faisceatu de His-Tawara avec cloison interventriculare. Candologen 1938; 1:61-76.

2. Wellens $\mathbb{H J J}$. Electroal Stimulation of the heat in the study and treamen of tachycardias. Uniwersity Parti Press, Ballimore, 1971

3. Gallagher J, Smith WM, Kassell JH, ef al: Role of Mahan fibers in cardiac arthyllamias an mat Circulation 1981;64:176-89.

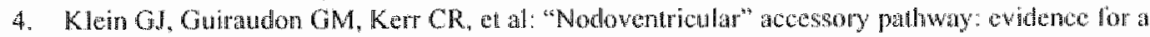
distimet acessory atroventicular pathway with atroventicular node-like properties. I Am Coll Cardiol 1988;11:1035-40

5. MoClelland JH. Wang X. Becknan KX, et al: Radiotrequency catheter ablation of Fight

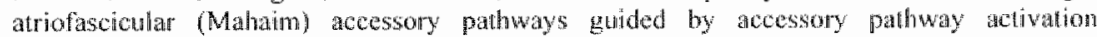
potentials. Circulation 1994,892655 - 66 .

6. Habsiguere M. Cauchenez B. Marcus $\mathrm{F}$, et al Characteristics of the ventricula insertion sites of accessoty pathways with anterograde decremental conduction properties. Circulation $1995,91: 1077-1085$

7. Tchou $\mathrm{P}$. Lehmonn MH, Jaxayen M, Akhtar M: Atriofascicular connection or a nodoventicular Mahain fiber? Elactrophysologic elucidation of the pathwy and associated reentrant circuit. Circulation 1988;77:837-48.

B. Guraudon CM, Guirudon GM, Klein Gil Histologic evidence for an acessory atrioveltricular patsway wh $A$ V-node-like morphology. Cilculation 1988;78(Supp/ II):40

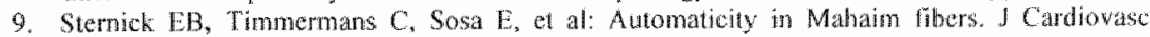
Electrophysiol $2004 ; 55: 738-744$ 
10. Haissaguere $M$. Warin JF, Le Metayer $P$, et al Catheter ablation of Mahaim fibers with presteryation of atrigyentricular notal conduction. Circulation 1990:82:418-427.

11. Tan HL, Witlkampl FHM. Nakagawa H, Derksen R. Atriofascicular accessory pathway. I Cardiovase Electrophysiol 2004:15:118.

12. Fung JWH, Chan HCK, Chan WWL, Sanderson JE: Ablation of the Mahaim pathway guided by noncontacl mapping J Cartiovase Electrophysiol 2002;13:1064.

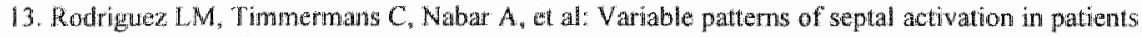
with left bundle branch block and hedrt failure. J Cardiovase Electrophysiol 2003;14:135-141.

14. Rudy $Y$. Prineiples of Slow Conduction in Cardiac Tissue: In Mazgalev TN, Tchou PJ (eds): "Alrial-AV nodal Electrophysiology: A View from the Millenum". Futura Publishing $\mathrm{Co}$. Ine., Armork, NY, 2000: 61-88. 


\section{Chapter 9}

\section{Dual conduction in a Mahaim fiber}

Eduardo Back Sternick*

Eduardo A. Sosa ${ }^{\dagger}$

Maurício I. Scanavaccał

Hein J.J. Wellens $\ddagger$

From the Biocor Instituto*, Belo Horizonte, Brazil, Instituto do Coração† da Faculdade de Medicina da Universidade de São Paulo, Brazil, Cardiovascular Research Institute $\ddagger$, Maastricht, The Netherlands

Journal of Cardiovascular Electrophysiology 2004;15: 1212-1215 


\begin{abstract}
An 8 year old girl is described suffering from an incessant non-sustained left bunde branch block (LBBBi)-like tachycardia refractory to antiarrhythmic drug therapy. An electrophysiologic study revealed the presence of a right sided accessory atriofascicular pathway. The episodes of non-sustaned tachycardia were found to be based upon al dual response in arrioventricular conduction over the Mahaim fiber to one $\mathrm{P}$ wave. No reentrant tachycardia could be induced. The arrhythmia was cured by catheter ablation targeting a Mahaim potential at the right lateral tricuspid annulus.

The findings can be explained by longitudinal dissociation in a single Mahaim fiber, a fiber distally diverging into two fibers with different conduction times, or (less likely) two closely located Mahaim fibers with different concuction times.
\end{abstract}




\section{INTRODUCTION}

In the presence of two connections between the atrium and the ventricle or dual AV nodal pathways, it is possible to have two QRS complexes after one simus $P$ Wave ${ }^{[-1 !}$.

Mahaim fibers, which are accessory atriovenmicular pathways with long conduction times and decremental properties, are considered as being composed of accessory $\mathrm{A}-\mathrm{V}$ nodal tissue ${ }^{2-16}$.

Here we describe a patient with a Mahaim fiber showing 2 QRS complexes after one sinus $\mathrm{P}$ wave.

\section{CASE HISTORY}

An 8 year old female child was referred because of an almost incessant tachycardia of 2 years duration. Onset of tachycardia was not related to specitio factors and was associated with palpitations. Echocardiography showed no abnormality. The 12-lead electrocardiogram (figure 1, left panel) showed episodes of non-sustained wide complex tachycardia with a LBBB-like configuration. During tachycardia a $1: 2$ relationship between the $P$ waves and the QRS complexes was present. In the absence of tachycardia the sinus beats showed a normal PR intervall $(0,12 \mathrm{sec})$, with a minimally preexcited QRS complex with an iS pattern in lead III (figure 2) and absence of septal q waves in the left leads. Twenty-four hour Holter monitoring showed most of the day repetitive bursts of tachycardia. There was a decrease in the number of episodes and beats per run during the night. Pharmacologic therapy was unsuccessful. Antiarthythmic drugs like verapamil, propranolol, and amiodarone did not change symptoms nor diminished the occurrence of tachycardia runs.

\section{METHODS AND RESULTS}

During electrophysiologic study, measurements during simus thythm revealed an AH interval of $80 \mathrm{msec}$; an HV of 25 to $30 \mathrm{msec}$, a His-right bundle branch (RBB) interval of $20 \mathrm{msec}$ and a QRS width of $90 \mathrm{msec}$. High right atrial pacing at increasing rates showed typical findings of a accessory pathway with long conducion times and decremental properties with progressive AH delay, reversal of the His bundle-right bundle depolarization, HV shortening and increasing preexcitation. Wenckebach block in the accessory pathway occurred with high right atrial pacing at $260 \mathrm{msec}$. The maximal increase in the anterograde conduction time of the Mahaim fiber was 140 msec. A proximal Mahaim (M) potential was recorded at the right lateral annulus with an atrium-Mahaim (AM) interval of 50 msec. We could also record a distal Mahaim potential in the right anteroapical region (figure 2). We observed a 1:2 P wave to QRS complex ratio when the sinus cycle length was shorter than $900 \mathrm{msec}$. This 1:2 ratio disappeared during atrial pacing at cycle lengthis below 

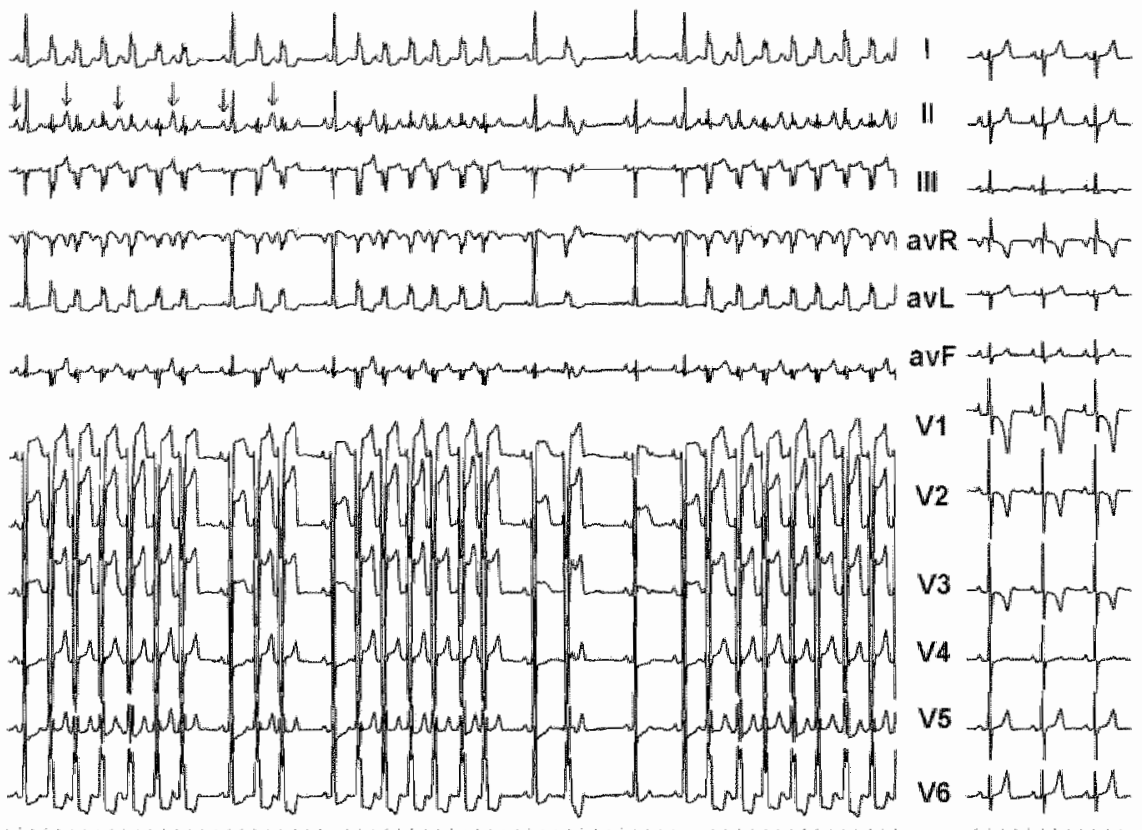

Figure 1: Lef pavel a 12 lead ECG during simus rhwh showng trequent apisodes of non-

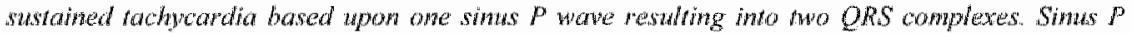

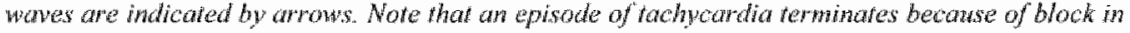
the "slow" Mahaim pathway. The QRS complex after the panse is a fusion complex between AV conduction oner the A-H node and the Maham fiber.

Right panel The 12-1ead ECG affer wheter ablation of the Mahain fiber. Candiac memory is presen in $M$, an and early precordhal heads. Paprer speed. $25 \mathrm{~mm} / \mathrm{sec}$.

$700 \mathrm{msce}$. Atrial pacing at faster rates resulted in a $1: 1 \mathrm{~A} \times \mathrm{V}$ conduction over the "fast" Mahaim pathway and only occasional beats with dual response were seen at the onset of atrial pacing drive. After atrial pacing we observed a transient suppression of the dual response pattern related to a slowed sinus rate because of the overdrive suppression effect of atrial pacing. With acceleration of the sinus rate the dual response pattern resumed. Response to intravenous adenosine was slowing of the $\mathrm{A}-\mathrm{V}$ conduction time and blocked $\mathrm{P}$ waves, with a gradual resumption of the dual response. During ventricular pacing no ventriculoatrial conduction was observed, and no reentrant tachycardia could be induced. Catheter ablation was performed targeting the proximal $M$ potential. Ten pulses of radiofrequency were deliwered and an irregular rhythm consistent with heat induced automaticity was seen followed by complete block in the Mahaim fiber. After ablation 1:1 A.V conduction ensued. Lead III now showed a qR pattern (figure 1 , right panel) 


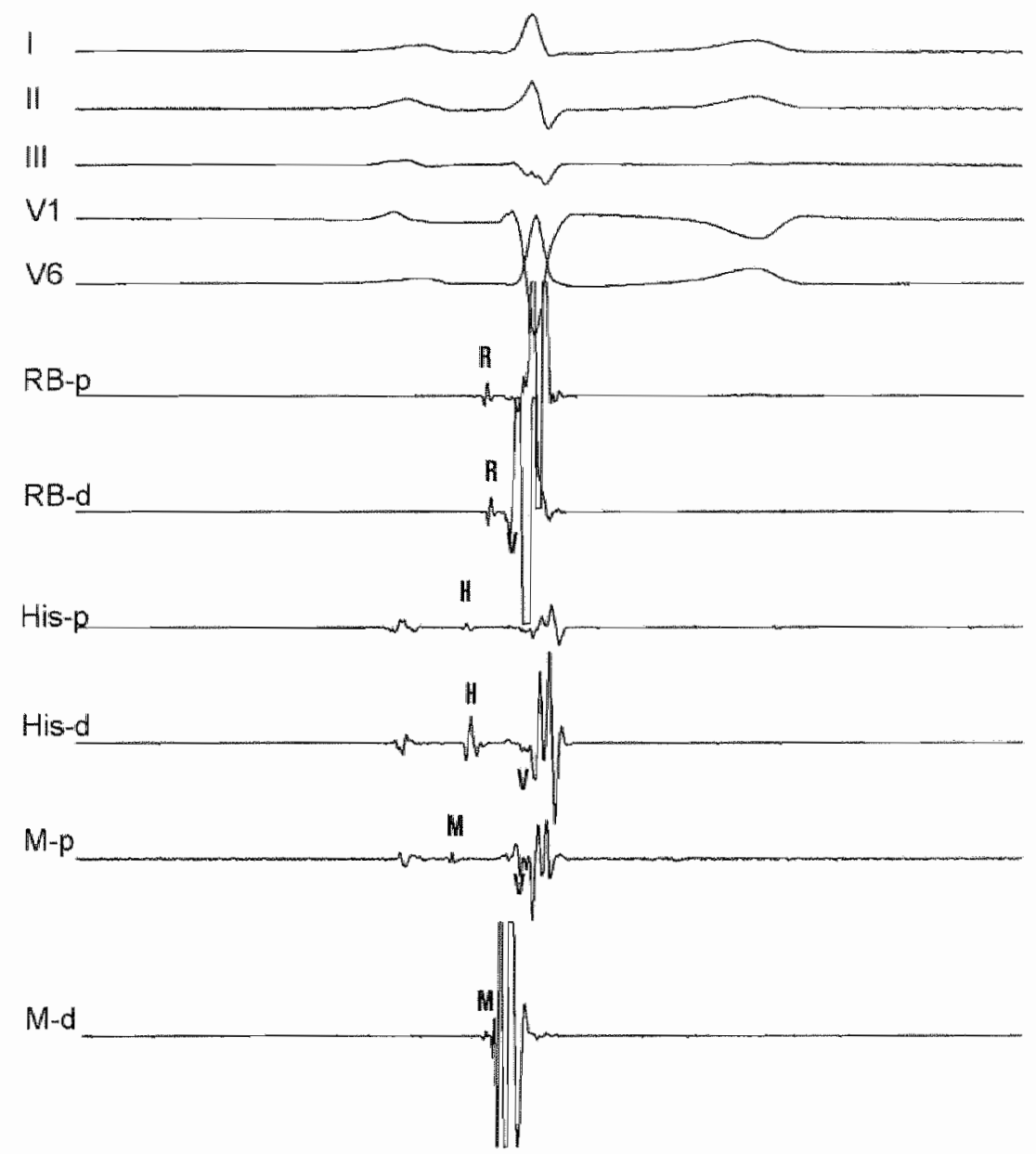

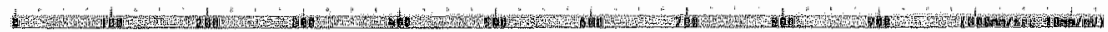

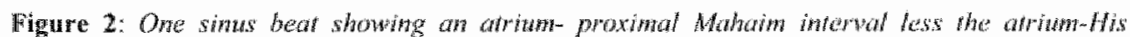

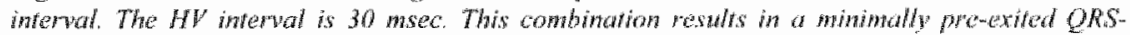

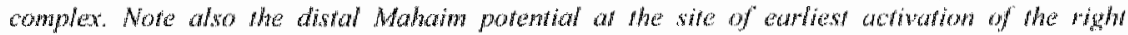
ventricte. Faper speed: $300 \mathrm{~mm} / \mathrm{sec}$.

Assessment of $A-V$ conduction showed $A-V$ conduction over the $A-V$ node without dual A-V node pathway.

\section{DISCUSSION}

Duality of conduction over Mahaim fibers because of longitudinal dissociation was 
$\frac{5}{3}$
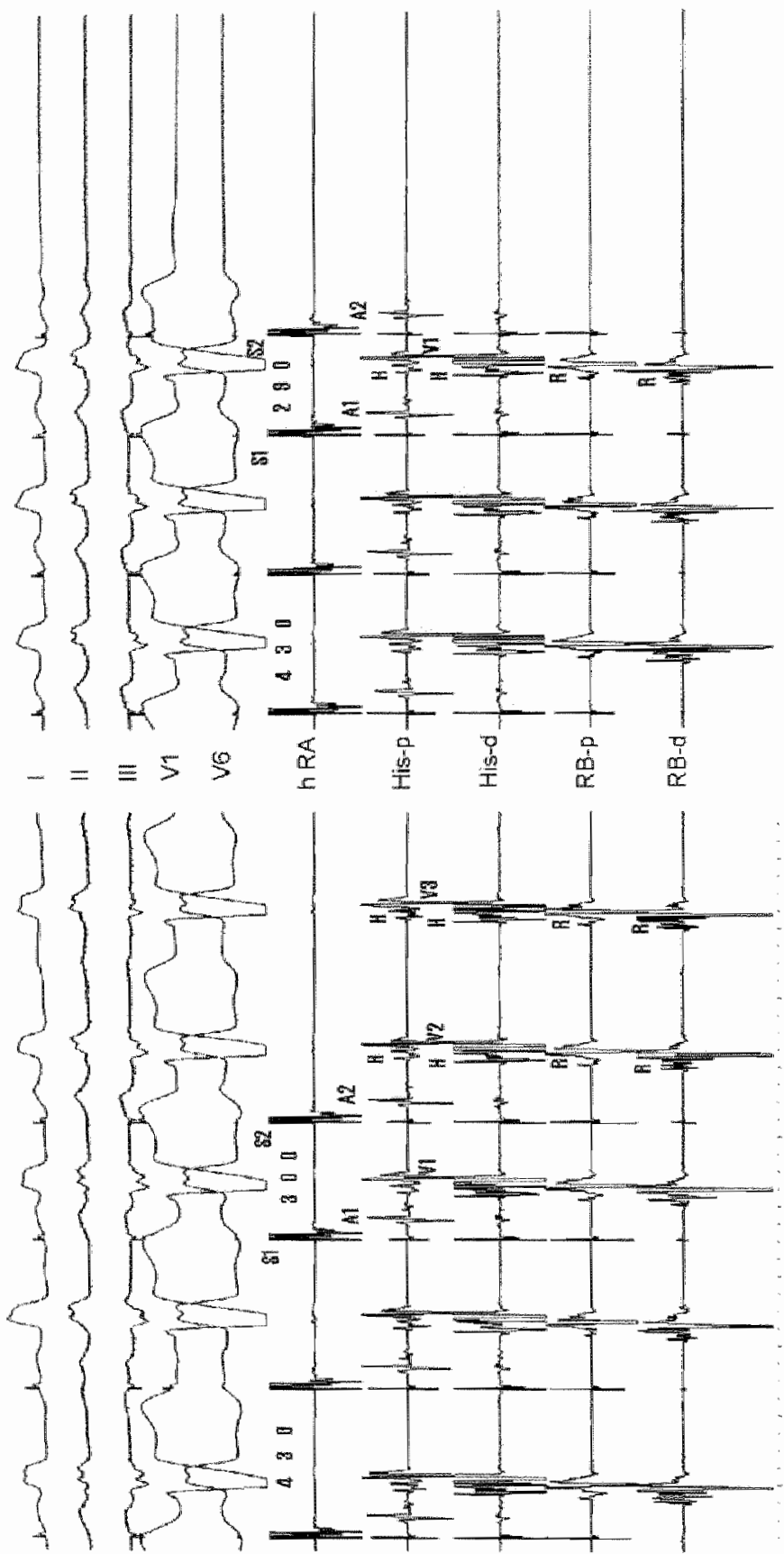


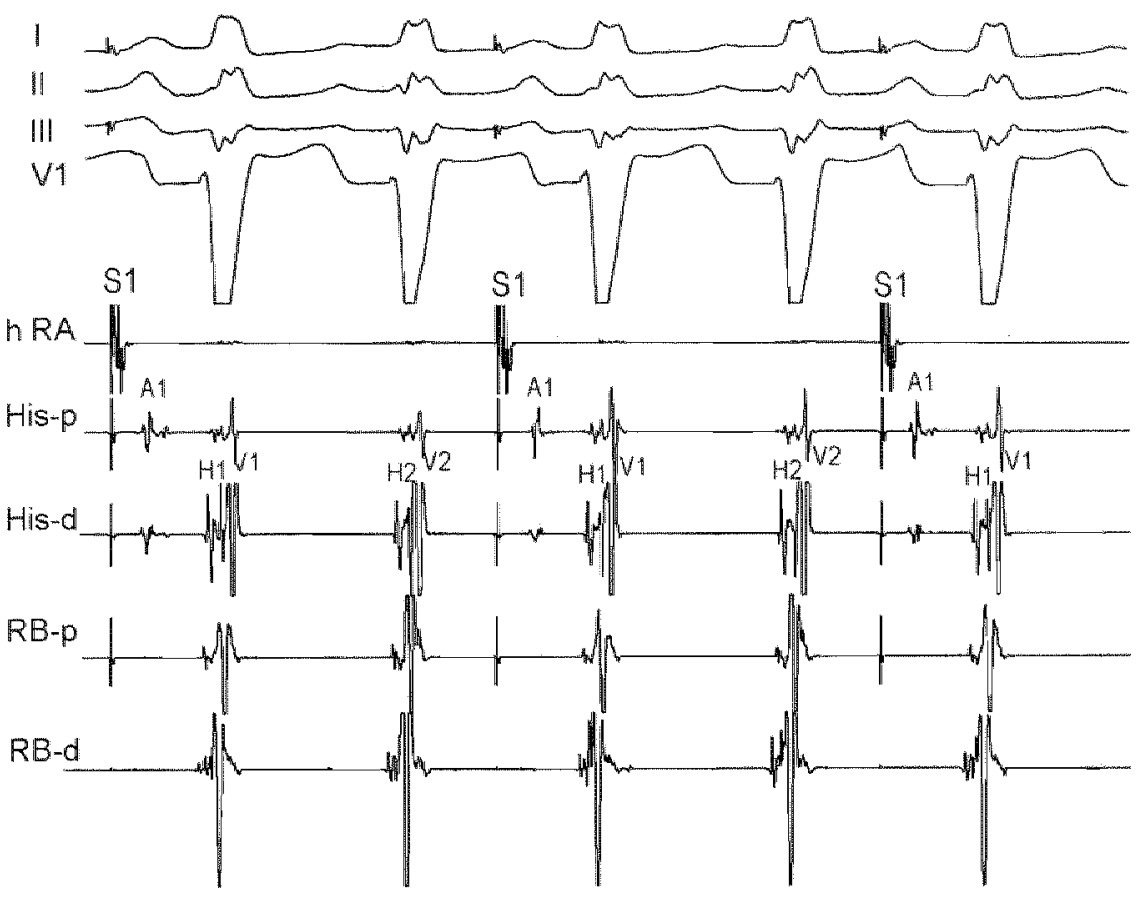

$\frac{0}{\frac{0}{8}}$

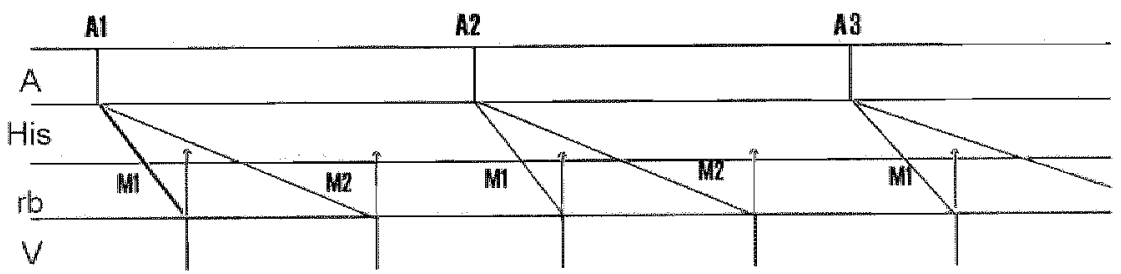

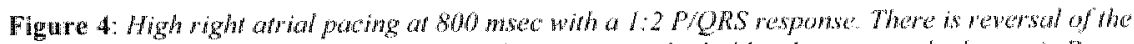

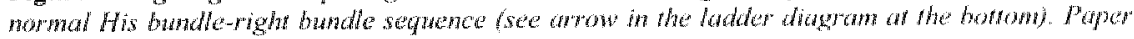
speed: $150 \mathrm{~mm} / \mathrm{sec}$. 
described to explain cycle length altenans during antidromic tachycardia in patients with Mahaim fibers. This concept was validated by showing a "jump" and a dual conduction patterm over the atriofascicular pathway during atrial extra stimulus testing ${ }^{1719}$. However, to the best of our knowledge there are no previous reports of spontaneous dual Mahaim conduction resulting in a 1 to $2 \mathrm{P} / \mathrm{QRS}$ ratio. From patients with nonreentrant tachycardia due to simultaneous conduction over dual A V nodal pathways we leamed that: 1 . they are often refractory to antiarrhythmic drug treatment. In fact tachycardia can be facilitated by the use of drugs; 2 . Usually no reentrant $A-V$ nodal tachycardia can be induced; 3 . They usually show absence of retrograde $\mathrm{V}$-A conduction. Without retrograde invasion into the "slow" pathway at the time of anterograde conduction over the "fast" pathway, conduction can proceed anterogradely over the "slow" pathway and can reach the wentricle a second time. Dual Mahaim conduction in our patient did only occur spontaneously during sinus rhythm or during right atrial pacing over an atrial cycle length range between 700 and $900 \mathrm{msec}$ (figure 4). One may speculate that at slow rates retrograde invasion into the "slow" pathway prevented anterograde conduction over this structure. We could not demonstrate anterograde block in the "slow" Mahaim with the atrial extra stimulus technique. Impulse conduction proceeded over either pathways or none (figure 3 ).

However during sinus rhythm episodes of a dual response tachycardia terminated by block in the "slow" Mahaim fiber (figure 1). We are not sure about the exact anatomic situation in our patient. Findings can be explained by a single Mahaim fiber with longitudinal dissociation resulting in dual pathways. We cannot exclude a fiber distally diverging into two fibers with different electrophysiologic properties. As shown in ligure 1 , the QRS complexes during 1 to 2 P-QRS conduction are not the same. These differences can be explained however by different degrees of aberrancy related to changes in the QRS interval.

Two closely located Mahaim fibers with different conduction times seem unlikely in view of the single Mahaim potential, and also because of the observation (figure 3) of block in both pathways on reaching a critical atrial premature beat interval, suggesting a proximal common pathway. Spontaneous enhanced automaticity may also occur in a Maham fiber ${ }^{20.21}$. This can be excluded in our patient by the strict I to 2 relation between the sinus $P$ wave and the QRS complexes. This was also seen during atrial pacing. A-V dissociation was never observed during the non-reentrant tachycardia.

Final proof was the relation between 1 to 2 conduction at critical sinus and atrial pacing rates.

\section{References}

1. Hosephson ME, Seides SE, Damato AN: Woll-Parkinson. Whate syndrone with 1:2 atrioventricular conduction. An I Cardiol 1976,37:1094-1096

2. Csapo O: Paroxysmal nonirentrant achycardia due to simulwateous corduction hrough dual atrioventriudan nodal pathways. Am I Cardiol 1979;43:1033-1045.

3. Suton FJ, Lee YC: Suptaventricular nonreentrant tachyeardia due to simultaneous conduction through duat atrioventricular nodal petteways. Am I Cardiol 1983;51:897-900.

4. Buss 1. Kratz I. Stegati $B$, at Unusual nechanism of $P R$ interval variation and nongentran supatentricular tachycandia as manifestation of simultaneous araterograde fast and stow conducton through dual atrioventricula nodal pathways. Pacmg Clin Electrophysiol
$1985,8,235-241$. 


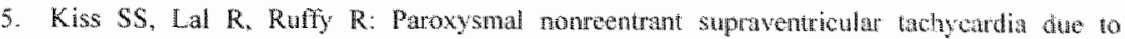
simultaneous fast and slow pathway conduction in owal atrioventicalar nodal pathways. 1 A.m Coll Cardiol 1987;10:456-461.

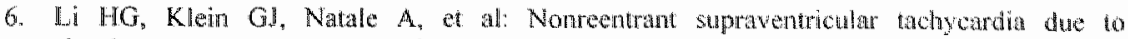
simultaneous conduction over fast and slow AV node pathways. Pacing Clin Elactrophysio $1994: 17: 1186-1193$.

7. Glotz de Lima G, Roy D. Talläic M, Dubuc M: One-towo atriowentricuar conduction causing nonreentant tachycardia: successful treatment wih ndotequency ablaton. Pacing Clin Electrophysiol 1998:21:1152*1154

8. Arena G. Bongioni M, Soldati E, et al: Incessant nonentrant tachycardia due to multiple nodal pathways treated by radiofrequency ablotion of the slow pathwys, Cardiowasc Electrophysiol 1999;10:1636-1642.

9. Kurchhof $P$, Loh $\mathrm{P}$, Ribbing $M$, Wasmer $K$ : lncessant supraventroular tachycurdia wilh constant 1:2 AW ratio: a longitudinally dissoctated artiowentricuar node? J Cardiowase Electrophysiol 2003;14:316-319.

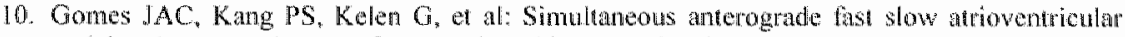
nodal pathway conduction after procainamide. An \Cardiol 1980;46:677-684.

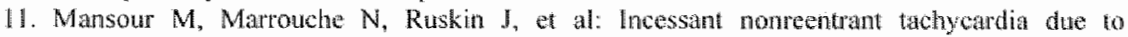
simultaneous conduction over dual atrioventricular nodal pathways mimicking anith fibrillation in patients referted for pulmonaty vein isolation. I Candiovase Electroptyssiol $2003 ; 14: 752-755$

12. Wellens HJJ. Electrical stimulation of the heart in the study and treatment of tactncardias. University Park Press, Baltimore, 1971.

13. Klein GI, Guiraudon GM. Kerr CR, et al: "Nodowentricular" accessory pathway: evidence for" a distinct accessory atrioventricular pathwa with atrioventicular node-like properties. I An Coll Cardiol 1988;11:1035-1040

14. MeCleltand JH, Wang X, Becknan $K J$, at: Radiofrequeney catheter ablation of right atrofascicula" (Mahain) accessory pathways guided by accessory pathway actwation potentials. Circulation 1994;89:2655-66.

15. Ellenbogen KA, Rogers R, Old W: Plwamacological characterization of conduction ower a Mahain fiber" evidence for adenosine sensitive conduction. Pacing Clin Electroplysiol $1989 ; 12: 1396-1404$.

16. Sternick EB, Gerken LM, Vrandecic MO: Appraisal of" "Mahaim" automatic lachycardia. I Candiovase Electrophysiol 2002;13:244-249.

17. Belhasen B. Mistahi D. Shapira 1 , Lanado $S$ : Longitudinal dissociation in an anomalous accessory atrioventricular pathway. An Heart J 1983;106:1441-1443.

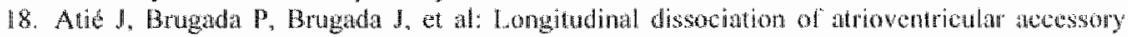
pathways. Am Coll Cardol $1991: 17: 161 \times 166$.

19. Okishige K. Friedman PL: New obserwatons on decremental atriofasciculan and nodofascicular fibers. implicstions for catheter abation. Pacing Clin Elactophysiol $1995 ; 18: 986-998$.

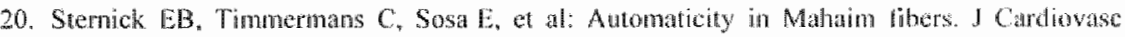
Electrophysiol 2004;15:738-744.

21. Sosa E, Scanavacea M: Repetitive, non-sustained whe QRS complex tachyedrda: what is the tachycardia mechanism? I Cardiovase Elecinophysiol $20011 ; 12977-8$. 


\section{Chapter 10}

\section{Appraisal of Mahaim automatic tachycardia}

Eduardo Back Sternick

Luiz M. Gerken

Mário 0 . Vrandecic

From the Biocor Instituto, Nova Lima, Brazil.

Journal of Cardiovascular Electrophysiology 2002;13:244-249 


\begin{abstract}
A series of four patients with right-sided accessory pathways with long conducting times and decremental properties is reported. All patients underwent radiofrequency catheter ablation and target areas were guided by a discrete "Mahaim" potental recorded at the lateral aspect of the tricuspid valve. A slow automatic and irregular rhythm with a QRS morphology similar to that of a fully preexcited QRS complex occurred during radiofiequency current delivery. The occurrence of so-called "Mahaim" automatic tachycardia heralded successful elimination of the accessory pathway in a manner similar to that of junctional automatic rhythm during slow pathway ablation in patients with A-V nodal reentrant tachycardia.

The observation of an automatic rhythm brought about during radiofrequency current ablation of a Mahaim accessory pathway is electrophysiologic evidence of the accessory A-V nodal behavior of this structure.
\end{abstract}




\section{INTRODUCTION}

There has always been an aura of mystery regarding Mahaim accessory pathways. Electrophysiologists long believed Mahaim accessory pathways represented nodofascicular or nodoventricular connections ${ }^{2}$. This misconception was challenged when patients with "Mahaim" fibers underwent surgical" and radiofrequency (RF) catheter ablation ${ }^{4}$ of the atrioventricular (A-V) node but remaned premexcited. Tchou et al shed light on the correct anatomic course, proving that the right atrium rather than the $A-V$ node is the proximal insertion of this accessory pathway. The same conclusion was reached when a surgical approach was attempted. In all cases, the authors found aright parietal accessory pathways rather than nodoventricular fibers. The same results were obtained in many centers worldwide ${ }^{7-11}$. Therefore, the concept of nodoventricular pathways was replaced by the atriofascicular pathway concept, and nodoventricular pathways became a rare item 11,12 . The issue of "Mahaim" automatic tachycardia has received litle attention in the literature, although there are many publications on the subject of atriofascicular pathways. The observation of automatic rhythms arising during RF ablation of atriofascicular pathways lead to an inevitable analogy with junctional automatic rhythms occurring during slow pathway ablation ${ }^{13}$. The am of the present article is to describe the electrophysiologic characteristics of these automatic thythms, which were documented in four patients with right-sided Mahaim-like accessory pathways.

\section{POPULATION}

Five patients having electrophysiologic diagnosis of atriofascicular accessory pathways according to criteria described elsewhere ${ }^{3}$, underwent catheter ablation at our Institution between 1996 and 2001. Ablation was performed successfully by targeting sites where "Mahaim" ("M") potential was recorded in 4 of 5 patients (Table 1). Other criteria, such as earliest stimulus-delta wave interval while pacing the right atrium at different sites close to the $\mathrm{A}-\mathrm{V}$ ring, were sought in 1 of 5 patients. Automatic thythms due to RF current delivery (Table 2) arose in all patients in whom an accessory pathway potential was recorded and catheter ablation was successful. Neither automatic rhythms nor "Mahaim" potential was recorded in the patient whose procedure was unsuccessful.

\section{RESULTS}

\section{Case reports}

Case 1: A 21-year-old girl with occasional paroxysmal tachycardia since age 12 years was referted to Biocor Institute for evaluation and treatment of substantial increased frequency of episodes despite previous oral antiarrhythmic therapy with sotalol and then with amiodarone. Echocardiogram disclosed Ebstein's anomaly with slight 
TABLE I

Clinical characteristics

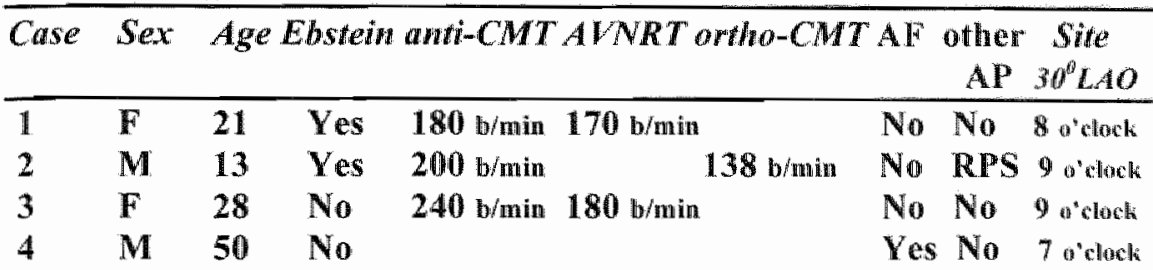

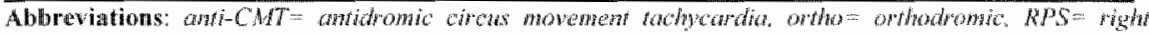

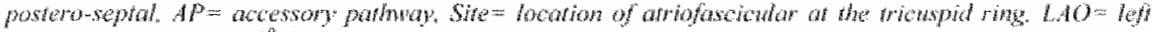

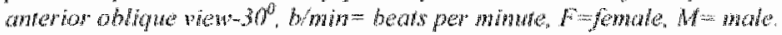

displacement of the tricuspid valve. Two different tachycardia patterns were induced during electrophysiologic study: typical A-V node reentrant tachycardia (AVNRT) and an antidromic circus movement tachycardia using an atriofascicular pathway as the anterograde limb and a slow A-V node pathway as the retrograde limb. Late right atrial extrastimuli that did not penetrate the $A-V$ node adwanced the timing of the subsequent QRS complex, proving that the accessory pathway was the anterograde limb of the circuit. A clear-cut accessory pathway potential was recorded at lateral aspect of tricuspid annulus ( 8 o'clock in the $30^{\circ}$ left anterior oblique (LAO) view) (figure 1). In all cases RF current was delivered using a 4-mm tip and thermistor large curve EPT (Boston Scientific Co., Watertown, MA, USA) 8-F ablation catheter. At 0,5 second after starting current

TABLE II

Ablation data

\begin{tabular}{rrrrrrrrr}
\hline \multicolumn{2}{c}{ Case } & M pot. Sheath & Temp & \multicolumn{4}{c}{ M.Automatic Tachycardia: RFp } \\
\multicolumn{1}{c}{} & & ${ }^{\circ} \mathrm{C}$ & Start & Last & min-maxCL \\
\hline 1 & + & SR02 & 70 & $0,2 \mathrm{~s}$ & $7,8 \mathrm{~s}$ & $360-720$ & 2 \\
2 & + & SR02 & 50 & $0,6 \mathrm{~s}$ & $140 \mathrm{~s}$ & $320-700$ & $4^{*}$ \\
3 & + & & 60 & $1,0 \mathrm{~s}$ & $30 \mathrm{~s}$ & $340-640$ & 2 \\
4 & + & SR01 & 60 & $3,0 \mathrm{~s}$ & $3 \mathrm{~s}$ & $\mathbf{4 2 0 - 5 4 0}$ & 2
\end{tabular}

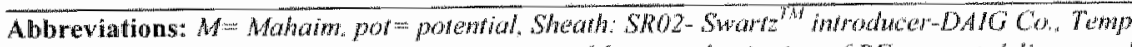

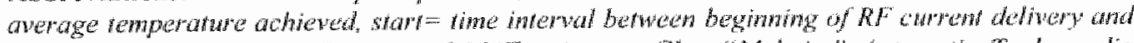

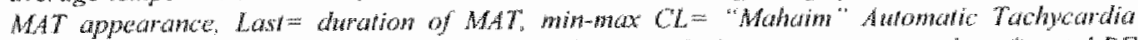

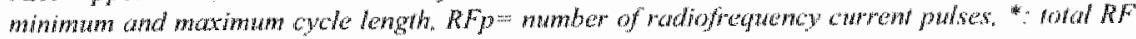
twhe of the 2 ablation sessions.

application, an irregular rhythm with QRS of varying width arose, resembling a fully pre-excited complex (figure 2). This salvo of non-sustained tachycardia lasted 7,8 seconds. 
The last three beats showed $\mathrm{V} \cdot \mathrm{A}$ conduction (A) and an arrial-pacing spike dissociated from the atrial potential. After tachycardia terminated a conducted non-3inus $P$ wave without pre-excitation was followed by wo other atrial paced beats with right bundle branch block (RBBB). No automatic tachycardia arose thereafter, and RF current was delivered for 60 seconds. The patient had no recurrence during 36-month follow-up. Case 2: 13-year-old boy with history of tachycardia since age 4 was referred to our institution for electrophysiologic evaluation. Various antiarthythmic drugs had failed to prevent recurrences. ECG showed a Wolfl-Parkinson-White pattern suggestive of right postero-septal location.

CASE

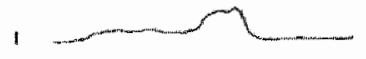

VI

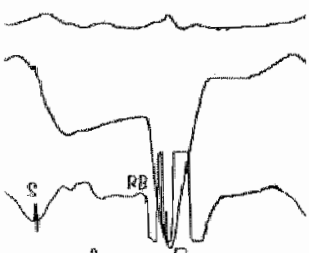

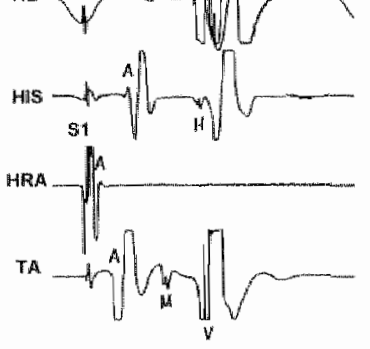

CASE 2

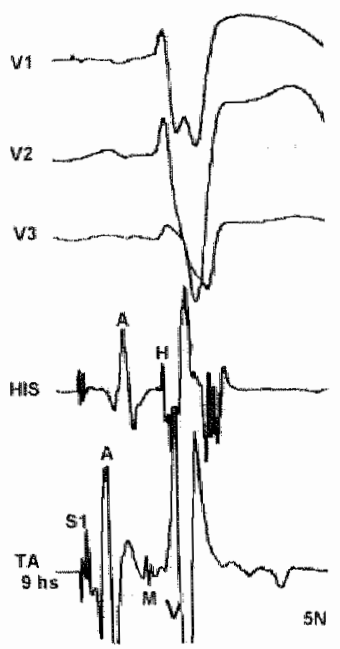

CASE 3
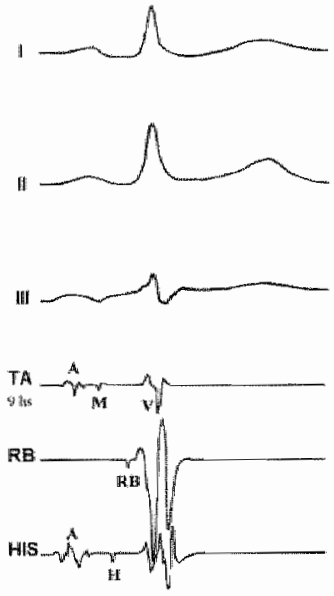

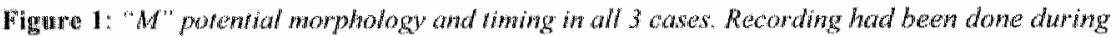
high laveral right atrial pacing in cases 1 and 2 .

Transthoracic echocardiogran was consistent with Ebstein's anomaly, without major hemodynamic impairnent. Two different tachycardias were induced at electroplrysiologic study: (1) 138 beats/min RBBB-like morphology orthodromic tachycardia, and (2) 190 beats/min LBBB-like morphology antidromic: tachycardia. After ablating the right postero-septal accessory pathway, features typical of atriofascicular pathway and induction of antidromic rachycardia were observed (figure 3). An " $\mathrm{M}$ " potential was recorded (9 o'clock in the LAO projectiony and targeted for ablation (figure 1). Irregular automatic tachycardia followed Rit application and preexcitation disappeared a few seconds after the current was delivered. Automatic thythm was recorded throughout current delivery ( 120 seconds), although the salvos progressively shortened. Less than 24 hours later, atriofascicular pathway conduction recurred. The patient underwent a second procedure I month later. The "M" potential was recorded at the same site, and there was no evidence of a righi posteroseptal palluway. 


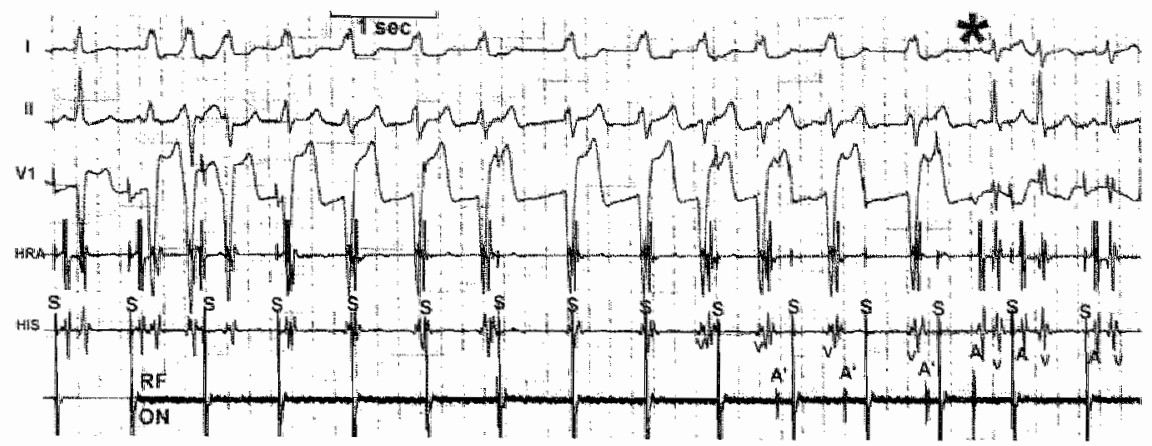

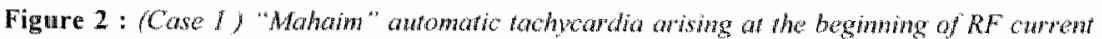

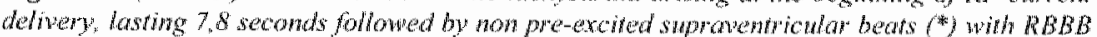
(Ebstein's anomoly).

Automatic rhythm arose again after RF current delivery. Preexcitation was abolished 3 seconds after RF application started, however, it was decided to terminate all automatic activity (figure 4), which required 20 seconds after starting RF current to achieve. Sinus rhythm then resumed. RF Energy was delivered for 2 minutes. Patient was arrhythmia-free during 46-month follow-up.

Case 3: A 28-year-old rural area primary school teacher was suffering many bouts of tachycardia, particularly while walking. Baseline ECG obtained on different days disclosed variable patterns ranging from minimal to full-blown preexcitation with a nomal PR interval. Transthoracic echocardiogram revealed no abnomalities. Spontaneous and induced tachycardia as well as atrial paced QRS complexes shared the same LBBB-like morphology. Two different tachycardias were induced at electrophysiologic study: (1) 180 beats/min with bystander preexctation AVNRT, and (2) 240 beats/min antidronic tachycardia. While mapping the tricuspid ring a discrete potential (figure 1) was recorded on its postero-lateral aspect and had been targeted for ablation. Autonatic rhythm started promptly after current deliwery (1 sec) and lasted for 30 seconds (salvos of unsustained irregular rhythm with LBBB-like morphology). The patient had an uneventful course and no arrhythmia recurrence during 5-year follow-up.

Case 4: A 50-year-man with preexcited atrial fibrillation, fast ventricular response and hemodynamic compromise was admitted to the emergency ward of Biocor Institute. It was his first episode of cardiac arthythmia. He was diagnosed as having ventricular tachycardia. DC cardioversion with 100 joules restored sinus rhythm, and the ECG was apparently normal. Transthoracic echocardiogram disclosed no abnomalities. Typical "Mahaim" physiology was present at electrophysiologic study. There was no $\mathrm{V}-\mathrm{A}$ conduction and no circus mowement tachycardia could be induced. 


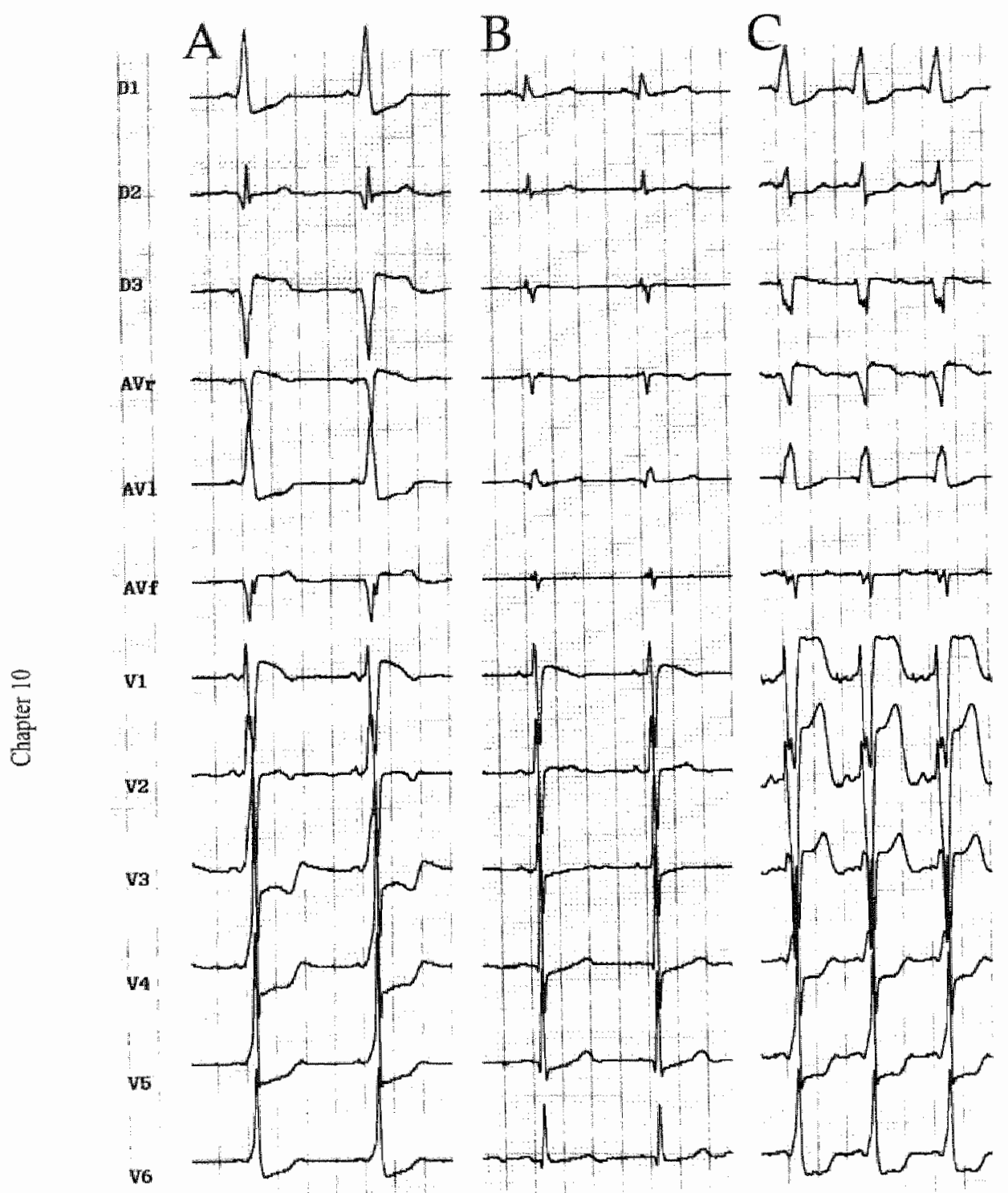

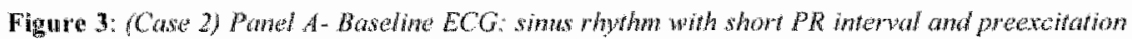

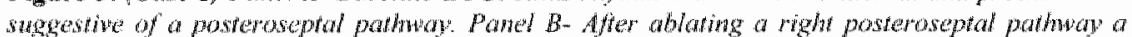

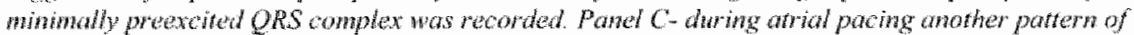

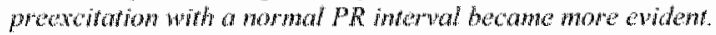

Sustained and self-terminating atrial fibrillation was induced by two extrastimuli delivered at the right atrum. Mean ventricular rate was approximately $220 \mathrm{~b} / \mathrm{min}$. The accessory pathway " $M$ " potential was recorded at the postero-lateral tricuspid anmulus ( 7 o'clock in the LAO projection) following an atrial deflection with an 
A/ $/$ ratio of 1:1 (figure 5). A short-lived burst of automatic tachycardia occurred five seconds after starting radiofrequency current deliwery. No additional automatic activity occurred during the last 50 seconds of ablation. Pation was arrhythmia-free during 2-month follow-up.

\section{DISCUSSION}

The results of previous reports on atriofascicular pathways are suggeslive of a proximal $A-V$ node like component and a distal bundle branch-like component, consistent with an accessory A-V conduction system, in most cases located at the postero-lateral tricuspid annulus, as in our cases, and occasionally elsewhere (interatrial septum ${ }^{14}$, mitral ring ${ }^{15}$ ).

Definite proof that such pathways are accessory $A-V$ nodes can only be obtained by fortuitous anatomic-pathologic assessment in patients with clinical diagnosis of atriofascicular pathways. Despite some reports on the occurrence of ectopic $\mathrm{A}-\mathrm{V}$ nodal tissue ${ }^{i k-20}$, sometimes with clear-cut features of an accessory $A-V$ node, with or electrophysiologic cortelation. In this context, characterization of automatic tachycardia while ablating atriofascicular pathways gains relewance.

Accelerated junctional rhythm is known as a response of the A-V node to thermal injury and common finding in RF ablation for AVNRT. The aceelerated thythms we and other electrophysiologists observed during RF current ablation in patients with like accessory pathways fits in that description of junctional rhythms. One conld argue that the thythms might be related to right ventricular heating. Davies ${ }^{21}$ observed without ventricular connections, there are insufficient data on its anatomic- Mahaim- like accessory pathways fits in that description of junctional rhythms. One could argue that the rhythms might be related to right ventricular heating. Davies ${ }^{21}$ observed less automaticity while ablating at the ventricular insertion. Based on the evidences invariably shown in all patients that there was a comparative cause-effect relationship between the occurrence of the automatic rhythm and successful ablation, the authors do not agnee that the rhythms occurned as a result of right ventricular heating.

Furthermore, RF current was allways delivered at a site with a large atrial potential (figure 1). Braun et al reported automatic rhythms during Mahaim accessory pathway ablation. They found that longer lasting thythms $(23 \pm 14$ vs. $8+14$ seconds/ $\mathrm{p}<0.05$ ) were associated with successful ablation.

Our observation was different: 2 of 4 patients developed short-lived Maham automatic tachycardia (case 1-7,8 sec and case 4-3 sec) and long-tem successful ablation. The occurrence of autornatic rhythms heralded successful ablation of the atriofascicular pathway in the same manner that junctional rhythin is associated with successful ablation and non-inducibility of $A$ VNRT. In three of our patients, Mahaim automatic tachycardia was no longer induced despite effective heating of the target area for as long as 60 seconds (case 2 ). Whether there is a relationship between complete termination of Mahaim automatic tachycardia by catheter ablation and persistent successful results is not known. This relationship was not present while ablating the slow $A-V$ nodal pathway. In other words: automatic junctional whythm can be induced by RF current application after successful ablation of AVNRT. Assuming atriofascicular pathways and $A-V$ nodes are related structures, the dissimilarify may be due to the size of the target structure. Posterior extensions of the $A-V$ node ${ }^{23}$ may be larger and/or longer than an 


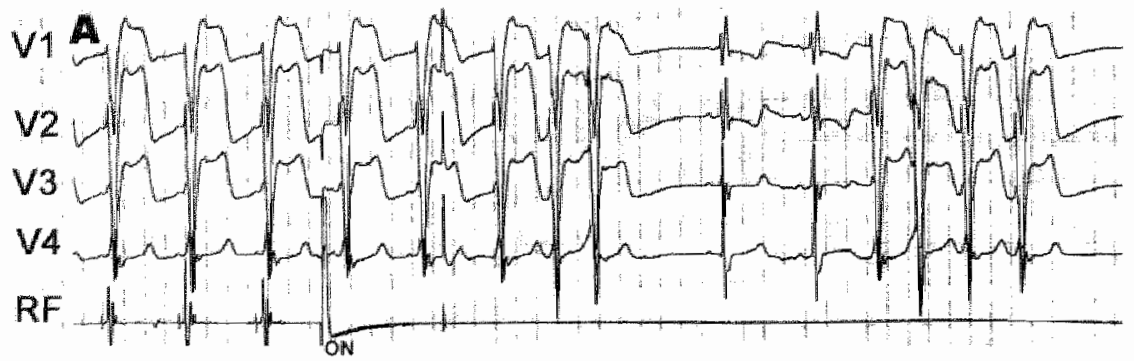

B

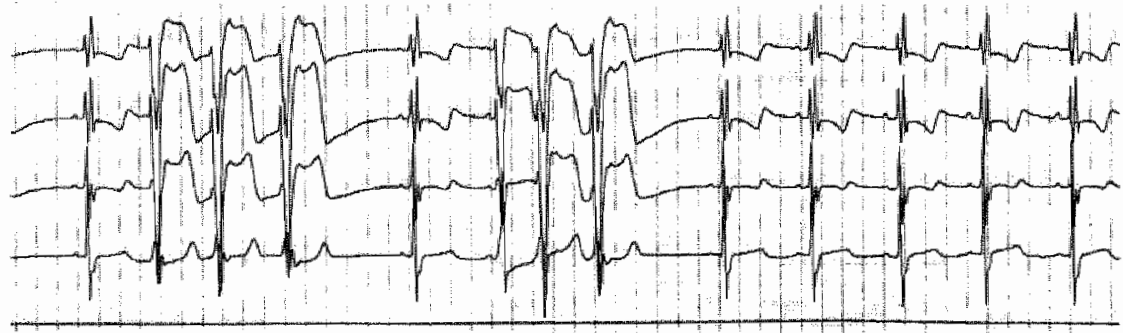

C

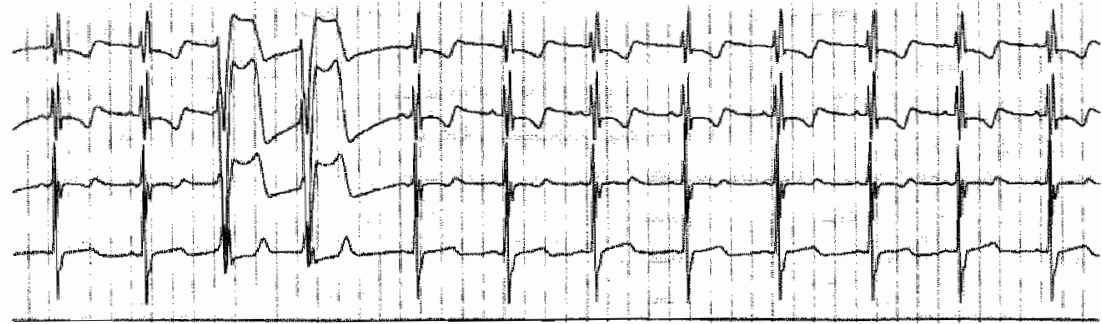

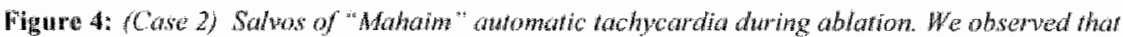

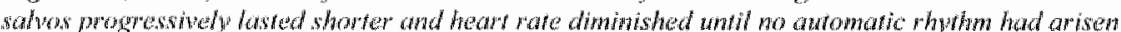

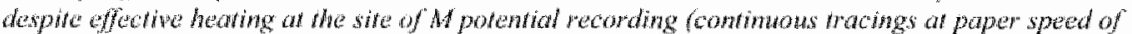
$25 \mathrm{minh} / \mathrm{wec}$

accessory $A-V$ node, accounting for viable $A-V$ nodall tissue that does not participate in the tachycardia circuit.

\section{Clinical implication}

Occurrence of "Mahain" automaticity during RF ablation of atriofascicular pathways seems to predict immediate success of the procedure. 

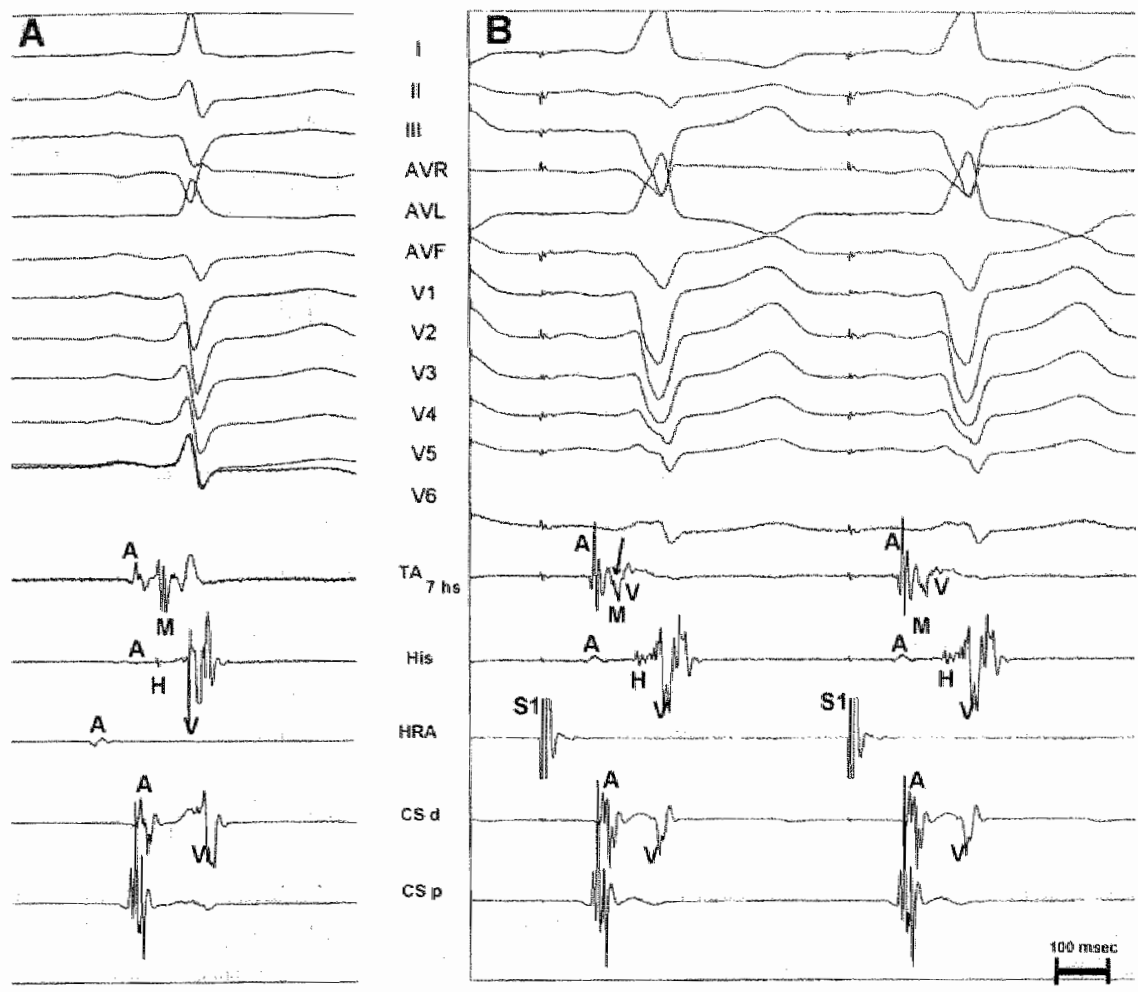

Figure 5: (Case 4) A- Simuthaneous recowding of the His bundhe potentiat amd the "M" poremial

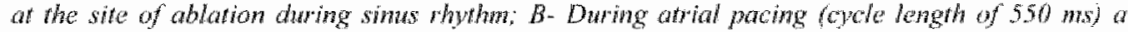
higher degree of preexcitation due fo protongaton of the AH internat occu's, while $A$ " $M$ " interval remans constant.

\section{CONCLUSION}

Heat induced automaticity is not observed while ablating regular accessory A-V pathways or normal atrial myocardium. Its occurrence due to RF ablation of atriofascicular pathways strongly suggests that the target structure might have $\mathrm{A}$ $\checkmark$ node like tissue.

\section{References}

1. Mahaim I. Benatt $A$ : Nouvelles recherches su les connexions stiperieures do ba banche gauche du faisceau de 1His-Tanara avec cloison interventriculaire. Cardiologid 1938;1:61.76.

2. Gallagher JI, Smith WM, Kassell JH, ef al: Role of Mahaim libers in cardac arthythmists in man. Circulation 1981:64:176-89.

3. Klein GJ, Guiradon GM, Kerr CR, at "Nodoventricular" accessory paltway evidence for a distinet accessory atrioventricular pathway with atrioventricular node-like properties. $\mathrm{d}$ Am Coll Cardiol 1988;11:1035-1040. 
4. Bhandari $A$, Morady $F$, shen EN, et al: Catheter-induced His bundle ablation in a patient with reentrant tachycardia associated with a nodoventricular tract. $₫ \mathrm{Am}$ Coll Cardiol 1984;4611616.

5. Tetou $P_{4}$ Lehmann MH. Jacayeri M, Akhtar Mr Atriofascicular connection or a nodoventricular Matuim fiber? Electrophysiologic elucidation of the pathway and associated reentrant circuit. Circulation $1988 \% 77.837 .48$

6. Murdock C, Letch JW, Klein JG, et all: Epicardial mapping in patients with "nodoventricular" accessory pathways. An J Cardiol 1991:68:208-214.

7. Brugada $J$, Martinez-Sanches $d$, Kuzmicic B, at al: Radiofrequency catheter ablation of atriofiscicular accessory pathways guided by discrete electrical potentials recorded at the tricuspid annulus. Pacing Clin Electrophysiol 1995;181388-94.

8. Mcclelland JH, Wang $X$. Becknan $K$, et al: Radiofrequency cathetar ablation of right atriofascicular (Mahaim) accessory pathways guided by accessory pathway activation polentials. Circulation 1994;89:2655-66.

9. Miller JM. Harper $\mathrm{OL}$, Rothman AS, Hsia HH: Radiofrequency catheter ablation of an. arroflascicular pathway during atrial fibrillation: a case report. I Cardiovasc Electrophysiol $1994,5: 846-53$.

10. Revault d'Allonnes $G$, Pavin $D$, Lebreton $H$, et al: Accessory pathways of decremental conduction (Maham Thers). Treatment by endocavitary ablation; apropos of 8 cases. Arch Mal Coeur Vaiss 1998;90:1637-43.

11. Kuck $\mathbb{K H}$, Siebeels J, Braun $\mathbb{E}$ : Electrophysiologic pecularities of nodoventricular accessory pathways with Mahaim-type preexcitation. J Am Coll Cardiol 1996;27(2-5uppl A):210.

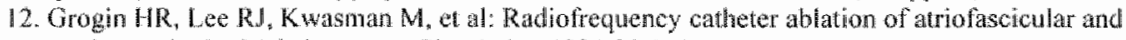
nodoventricular Mahaim tracts. Circulation 1994;90:272-81.

13. Boyle $\mathrm{NG}$, Ansetme F, Monahan $\mathrm{K}$, et at: Origin of junctional thythm during radiofrequency ablation of atriowentricular nodal reentrant tachycardia in patients without structural heart discase. Am I Cardiol 1997:80:575-80.

14. Petnado R, Merino JL, Ramirez, Echeverría I: Decremental atrofascicular accessory pathway with bidirectionall conduction: clefineation of atrial and ventriculat insertion by radiofrequency current application. I Cardiovasculac Electrophysiol 2001;12:489-492.

15. Huchy J, Schickel S, Jörger U, et al: Electrophysiologic characteristics and radiofrequency ablation of conceated nodofascicular and left anterograde atriofascicular pathways. J Cardiovasc Electrophysiol 2000; $11: 211-217$.

16. Guiraudon CM. Guiraudon GM, Klein G: Histologic evidence for an accessory atrioventricular pathway with AV-node-like morphology. Circulation 1988;78(Suppl II): 40.

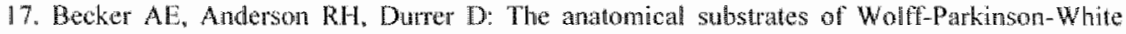
syndrome: a dinico-pathologic corretalion in 7 patients. Circulation $1977,57: 870-879$.

18. Epstcin MR. Saul JP. Weindling $\mathrm{SN}$, et al: Atrioventricular reciprocating tachycardia involwing twin atroventricular nodes in patients with complex congenitat heart disease. J Cardiowase Electrophysiol 2001:12:671-679.

19. Nogani A, Sugula M, Tomita $T$, et al: Novel form of atrial tacthycardia originating at the atroventricular annulus. Pacing Clin Electrophysiol 1998;21:2691-94.

20. Gollob $\mathrm{MB}$. Bharati $\mathrm{S}$. Swordiow $\mathrm{CD}$; Acessory atrioventricular node with properties of a typical accessory pathway: Anatomicelectrophysiologic correlation. I Cardiovasc Electrophysiol 2000;11:922-6

21. Duryes DW: Treatment of "Mahaim" tachycardias by radiofrequency catheter ablation. In Camm I, Lindemans FW (cds.): Transvenous Defibrillation and Radiofrequency Ablation. Futura Publishing Co. Inc., Armonk, NY 1995:199.208.

22. Bratan E. Sicbels J. Volkmer M, et al: Radiofiepuency-induced preexcited automatic rhythm during ablation of accessory pathways with Mahaim-lype preexcitation: does it predict clinical outconte? Pacing Clin Electrophysiol 1907;20(4) Part 11:1121.

23. Medkour D. Becker AE, Khalife K, Billette $J$ : Anatumic and functional characteristics of a slow posterior AV nodal pattway. Role in dual-pathway physiology and reentry. Circulation $1998 ; 98: 164-174$. 


\section{Chapter 11}

\section{Automaticity in Mahaim fibers}

Eduardo Back Sternick*

Carl Timmermans"

Eduardo A. Sosat

Fernando E. Cruz Filho ${ }^{\ddagger}$

Luz-Maria Rodriguez ${ }^{\mathbb{}}$

Luiz M. Gerken*

Mauricio I. Scanavaccat

Márcio L. Fagundesł

Sávia C. Buenot

Mário O. Vrandecic*

Hein J.J. Wellens ${ }^{\circledR}$

From the Biocor Instituto*, Belo Horizonte, Brazil, Instituto do Coração’ da Faculdade de Medicina da Universidade de São Paulo, Brazil, University Hospital ${ }^{\natural}$, Maastricht, The Netherlands, Instituto Nacional de Cardiologia Laranjeiras, Ministério da Saúdeł, Rio de Janeiro, Brazil. 


\begin{abstract}
Introduction: Automatic rhythms associated with Mahaim fibers usually occur during radiofrequency catheter ablation. The incidence and significance of spontaneous automaticity in Mahaim fibers is unknown.

Methods and Results: We found spontaneous automatic rhythms in $5(12,5 \%)$ of 40 patients with Mahaim fibers, referred for non pharmacologic therapy because of recurrent episodes of symptomatic tachyarrhythmias, usually antidromic circus movement tachycardia (33 out of 40 patients). Three were female and 2 male. Mean age was $15 \pm 7$ years as compared to $26 \pm 13$ years of the patients without automaticity $(\rho=0.09)$. Three patients had both antidromic tachycardia and asymptomatic spontaneous automatic rhythms, recorded during ambulatory electrocardiography (1 patient) or electrophysiologic study ( 2 patients). In 2 patients the automatic rbythm triggered antidromic tachycardia. Two other patients had non sustained repetitive episodes of wide QRS tachycardia due to automaticity arising in the Mahaim fiber, without antidromic tachycardia. All automatic rhythms were abolished by successful catheter ablation of the Maham fibers.

Conclusions: Spontancous automaticity occurred in $12.5 \%$ of our Mahaim patients and may trigger antidromic tachycardia. Spontaneous automaticity which is not seen in rapidly conducting accessory pathways is another argument for the presence of an $A-V$ nodal like structure in Maliaim fibers.
\end{abstract}




\section{INTRODUCTION}

Automatic impulse formation originates in cells with spontaneous diastolic depolarization. Those cells are found in the sinus node, the A-V node, the coronary sinus ostium, and the crista terminalis. In spite of the lack of conclusive anatomic-pathologic data, there are strong electrophysiological arguments suggesting that Mahaim fibers contain accessory AV nodal tissue such as 1) slow and decremental anterograde conduction ${ }^{2}$, 2) a structure with a proximal A-V nodal component and a distal bundle branch-like component, both with His bundle-like potentials ${ }^{3}, 4$ ) conduction block in response to adenosine and 4$)$ heat induced automaticity during radiofrequency catheter ablation ${ }^{6}$. According to the literature spontaneous automaticity arising in rapidly conducting accessory pathways is a very rare finding 7.8 . Spontaneous activity arising in Mahaim fiber has been reported only twice, 10 and its significance is unknown. Our aim therefore is to describe spontaneous Mahaim automaticity in a cohort of 40 patients with Mahaim fibers.

\section{METHODS}

\section{Definitions}

Every patient showed a decrementally conducting afrio-ventricular bypass tract with progressive atrio-ventricular and $\mathrm{AH}$ interval prolongation coupled with a decreasing HV interval leading to a greater degree of ventricular pre-excitation with a left bundlle branch-like morphology during atrial pacing ${ }^{2}$. The His bundle deflection was inscribed after the right bundle deflection during maximal preexcitation. At maximal ventricular preexcitation, there was a constant QRS-His relationship without further changes on shortening the atrial pacing cycle length.

\section{Study Population}

We retrospectively studied 40 consecutive patients from 4 Institutions having accessory pathways with long anterograde conduction times and decremental properties during sinus thythm, with tachycardia with anterograde conduction over the decremental pathway, and in whom ablation of the accessory pathway was performed.

There were 24 females and 16 males, with a mean age of $24 \pm 12$ (range 8 (o 80 ) years. Refertal for electrophysiologic assessment was because of a preexcited tachycardia over the Mahaim fiber in 33 patients (table I). Ebstein's disease was diagnosed in 8 patients (20\%). In 32 patients the atrial insertion of the fiber was identified by localizing a discrete accessory pathway potemial, and in 8 patients by assessing the shortest $\mathrm{A}-\mathrm{V}$ interval during atrial pacing at different sites. All patients underwent successful surgical $(\mathrm{n}=2)$ or radiofrequency catheter ablation $(n=38)$. RF ablation was done from the atral end in 32 patients, and in 6 patients from the ventricular end after righi ventricular pacemapping. Five patients (cases $9,18,22,33$ and 36 ) showed spontaneous automaticity (table I).

\section{Statistical Analysis}

Values are given as mean + standard deviation. The significance of differences ( $P$ 
$<0.05$ ) between groups of clinical, electrocardiographic or electrophysiologic parameters was assersed by Student's t test or Fisher's exact test.

\section{RESULTS}

\section{Spontaneous Automaticity}

The 5 patients with spontaneous automaticity were younger than the 35 patients without spontaneous automaticity $(15 \pm 7$ ws. $26 \pm 13$ years $)$ but the difference did not reach statistical significance $(P=0,09)$. Three were male and 1 had Ebstein's disease. None of the 5 patients had an associated rapidly conducting AP. Although the number of patients are too small for comparison, clinical presentation with antidromic tachycardia did not differ among Mahaim patients withoul spontaneous automaticity $(82 \%)$ and those $60 \%$ having it (3 out of the 5 patients). In those five patients $(12,5 \%)$ spontaneous automatic rhythms were found with the same QRS complex configuration as during maximal preexcitation. Mahaim automaticity rates ranged from a slow thythm occurring during the night, ind istinguishable from an accelerated idioventricular thythm (AIVR) (cycle length $=900 \mathrm{msec}$, to nonsustained repetitive tachycardia with a cycle length of 360 msec (table II). Three patients referred because of an antidromic tachycardia with anterograde conduction over the Mahaim fiber also showed spontaneous Mahaim automaticity. QRS complex configuration was identical during the reentrant and the automatic rhythm (figure 1). In one patient (case 36) episodes of asymptomatic slow automatic rhythm resembling AIVR occurred at a slower rate during the nighttime and at a faster rate during daytime (figure 3 ). In wo patients (cases 18 and 22) automaticity was the clinical problem. Those patients sought medical treatment because of a long history of nonsustained palpitations. Both patients showed repetitive bursts of nonsustained tachycardia with a left bundle branch block configuration. There was VA dissociation during the majority of the episodes, but in case 18 some tachycardias showed $2: 1$ retrograde $V$-A conduction over the $A-V$ node. Automatic thythm was recorded in all patients during the electrophysiologic study before the administration of isoproterenol. During its infusion there was an increase in both rate and duration of the episodes. The effect of isoproterenol was less pronounced in both patients with repetitive nonsustained automatic runs. In those 2 patients, there were only a slight increase in automaticity rate. Electrophysiologic validation of the site of arigin of this automatic rhythm included the recording of a $M$ potential preceding the preexcited QRS complex and retrograde depolarization of the right bundle followed by the His bundle, In patient 9 (figure 2 ), there was $V$-A dissociation during the automatic thythm.

During atrial overdrive stimulation there was a transient suppression without entramment, acceleration nor persistent termination of the tachycardia nuns in cases 18 and 22. Following stimulation tachycardia resumed almost immediately in cases 18 and 22 . 
TABLE I

Clinical data of patients with Mahaim Fibers

\begin{tabular}{|c|c|c|c|c|c|c|c|c|}
\hline Case & Sext & Agge & Site & Clinical arhythmi & $\begin{array}{l}\text { Therapy } \\
\text { sRFPRFd }\end{array}$ & $\begin{array}{l}M A T \\
\mathrm{AFp}\end{array}$ & WPIA & $\mathrm{CBT}$ \\
\hline 甚 & $F$ & 31 & L & Antididromic CMT/Mahaim & 5 & & & \\
\hline 2 & $\mathrm{~F}$ & 32 & L & Antidromic CMTMMahaim, AYNRT & $\mathrm{RFp}$ & Yes & & \\
\hline $3^{*}$ & $F$ & 19 & $\mathrm{PL}$ & Antidromic CMTMaham & RFp & Yes & & \\
\hline $4^{*}$ & $F$ & 21 & PL & $\begin{array}{c}\text { Antidromic CMTMahaim } \\
\text { Ontodromic CMT }\end{array}$ & $\begin{array}{l}\mathrm{RFp} \\
\mathrm{RFp}\end{array}$ & Yes & & RPS \\
\hline $5^{*}$ & M & 13 & PL & Antidiomic CMTMaham & $\mathbb{R F} \beta$ & Yes & RPS & \\
\hline $\mathbb{b}$ & $M$ & 52 & PL & Preexcited atrial fibrillation & RFp & Ves & & \\
\hline $7^{*}$ & $F$ & 19 & PS & Antidromic CMT/RAS AP & $\mathrm{RFp}$ & No & RAS & \\
\hline 8 & $F$ & 22 & P & fast palpitations & FiFp & Yes & & \\
\hline 9 & M & 21 & $L$ & Antidronaic CNT/Mahaim & $\mathbf{R F p}$ & Yes & & \\
\hline 10 & $F$ & 23 & $L_{0}$ & Antidromic ChT/Maham & BFp & Yes & & \\
\hline 11 & $M$ & 80 & PL & AVNPT + Mahaim bystande & FFp & Yes & RPS & \\
\hline 12 & $\mathrm{~F}$ & 19 & A & Antidromic CMTMahaim & $\mathrm{AFp}$ & Yes & & \\
\hline 13 & $F$ & 23 & $\mathrm{~L}$ & Antidromic CMTMMahaim & $\mathrm{RFp}$ & Yes & & \\
\hline 14 & $F$ & 25 & $\mathrm{PL}$ & Antidromic CMT/Mahaim & $\mathrm{RFp}$ & Yes & & \\
\hline 15 & M & 35 & $L$ & Antidromic CMTMahaim & RFp & Yes & & \\
\hline 16 & $\mathrm{~F}$ & 42 & $L$ & Antidromic CMTMatraim & RFp & Yes & & \\
\hline 17 & $\mathbb{F}$ & 23 & L & Antidromic CMTMahaim & RFp & Yas & & \\
\hline 18 & $F$ & $B$ & $\mathrm{AL}$ & Mahaim automaticity & $\mathrm{RF}_{p}$ & Yes & & \\
\hline 19 & M & 30 & $\mathbb{P L}$ & Antidnomic CMT/Mahaim & $R F p$ & Yes & & \\
\hline 20 & $\mathrm{~F}$ & 27 & $L$ & Antidromic CMIT/Mahaim & RFp & Yes & & \\
\hline 21 & $F$ & 19 & $\mathrm{~L}$ & Antidromic CAMTMahaim & RFp & Yes & & \\
\hline 22 & M & 12 & MIS & Mahatin automaticity & $\mathrm{RFp}$ & No & & \\
\hline 23 & $F$ & 39 & L & Antidromic CMTMahaim & RFp & Yes & & \\
\hline $24^{*}$ & $\mathrm{~F}$ & 12 & $\mathbb{L}$ & Antidromic CMT/Mahaim & RiFp & Na & RL & \\
\hline 25 & F & 15 & $\mathrm{ML}$ & AVMRT +Mahaim bystander & RFp & Yes & & \\
\hline 26 & $M$ & 13 & $\mathrm{~L}$ & Antidromic CMTMMahaim & RFp & Yes & & \\
\hline 27 & $M$ & 15 & $A$ & Antidromic CMTM Mahaim & RFp & Yes & & \\
\hline 28 & $F$ & 25 & $L$ & Antidromic CMTMahaim & RFp & Vas & & \\
\hline $29^{*}$ & $M$ & 17 & $L$ & $\begin{array}{c}\text { Antidromic CMTM/Mahairn } \\
\text { Ontodromic CMT }\end{array}$ & RFp & Yes & $\mathrm{RPS}$ & RL \\
\hline 30 & M & 18 & $L$ & Antidromic CMTMahaim & RFp & Ves & & \\
\hline 31 & $\mathrm{~F}$ & 45 & $\mathbb{L}$ & Antidromic CMT/Mallaim & RF $\mu$ & Yas & & \\
\hline 32 & $M$ & 24 & L & Antidromic CMTMathaim & RFd & & & \\
\hline $33^{*}$ & $\mathrm{M}$ & 11 & $L$ & Antidromic CMT Mabain & $\$$ & & & \\
\hline $34^{*}$ & $M$ & 26 & L & Antidromic CMTMaham & RFd & & & L.L \\
\hline 35 & M & 25 & 然 & Antidromic CMT/Mahaim & RFd & & & \\
\hline 36 & $\mathrm{~F}$ & 26 & $\mathrm{P}$ & Antiduomic CHT Malualim & RFA & & & \\
\hline 37 & $F$ & 31 & L & Aritidromic CMTMahaim & RFo & & & \\
\hline 38 & $F$ & 22 & $L$ & Antidromic CMTMaham; AVNRT & $\mathrm{RF}_{\mathrm{p}}$ & Yes & & \\
\hline 39 & $F$ & 17 & $L$ & Antidromic CMT/Malsaim & RFp & Yes & & \\
\hline 40 & M & 17 & $p$ & Antidromic CMT/Maham & $\mathrm{AF}$ & Yes & & \\
\hline
\end{tabular}

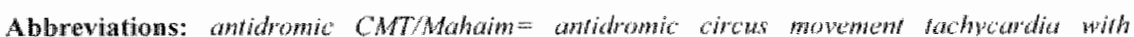

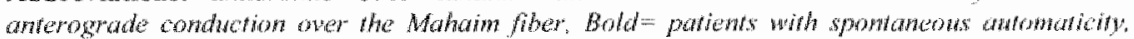

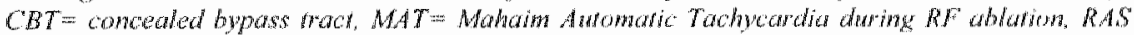

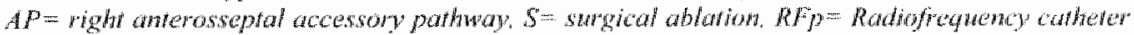

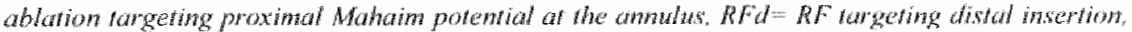

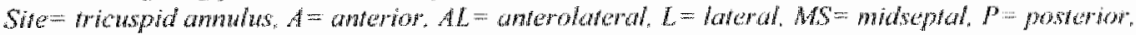

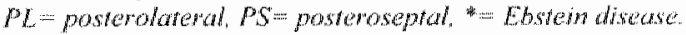




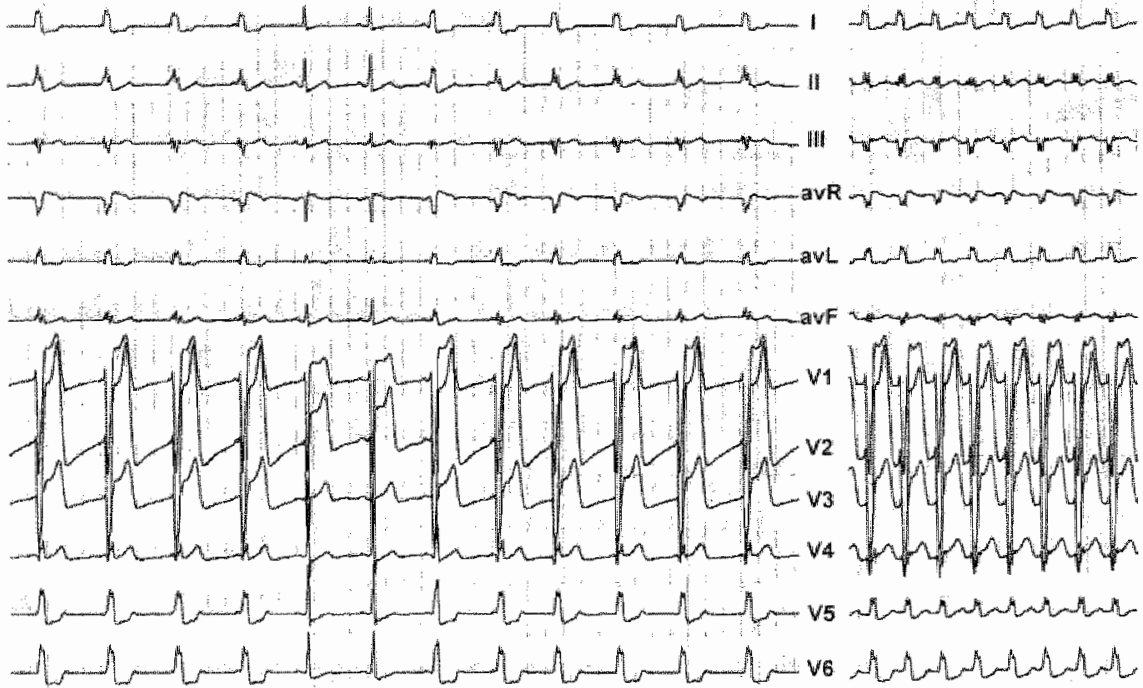

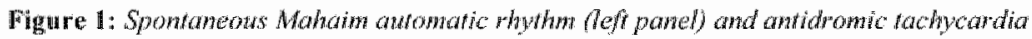
showitug the satne QRS complex mowphology (right panel).

\section{TABLE II}

Electrophysiologic data of the patients with spontaneous automaticity

\begin{tabular}{|c|c|c|c|c|c|c|c|c|}
\hline ns & Case & Shated arrhythmia & $\begin{array}{c}\text { AM } \\
\text { Cl range (rinec) }\end{array}$ & $\begin{array}{c}\text { Diagrinosis } \\
\text { at }\end{array}$ & $\begin{array}{l}\mathrm{CMI} \\
\mathrm{ByM}\end{array}$ & $\begin{array}{c}\text { Isruptel } \\
\text { Msceleration }\end{array}$ & $\begin{array}{l}\text { WA conduction } \\
\text { Mahaim } / \mathrm{M} \text { node }\end{array}$ & $\mathrm{MAT}$ \\
\hline 1 & 18 & antomatic runs & $550-450$ & $\mathrm{ECO}$ & & $*$ & not no & $y=s$ \\
\hline 2 & 22 & antomatic nus & $360-400$ & $\mathrm{ECG}$ & & $*$ & nolyes & no \\
\hline 3 & 9 & $\mathrm{a} \cdot \mathrm{CM}^{\mathrm{n}} \mathrm{I}^{\mathrm{n}}$ & 720.580 & EPS & yes & $x=$ & no $/$ yes & yes \\
\hline 4 & 33 & aCMT" & $840-550$ & Holter ${ }^{*}$ IPS & yes & *a: & nol yes & \\
\hline 5 & 30 & $\mathrm{~A}-\mathrm{MMT}$ & $900-5 y 0$ & Holter/HPS & & 䊉泣 & no / ges & \\
\hline
\end{tabular}

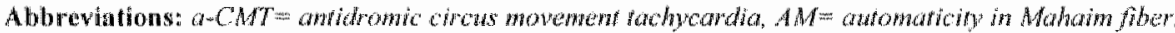

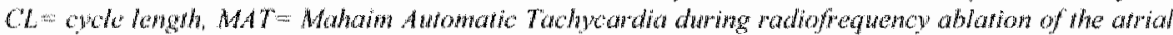

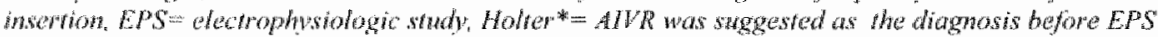




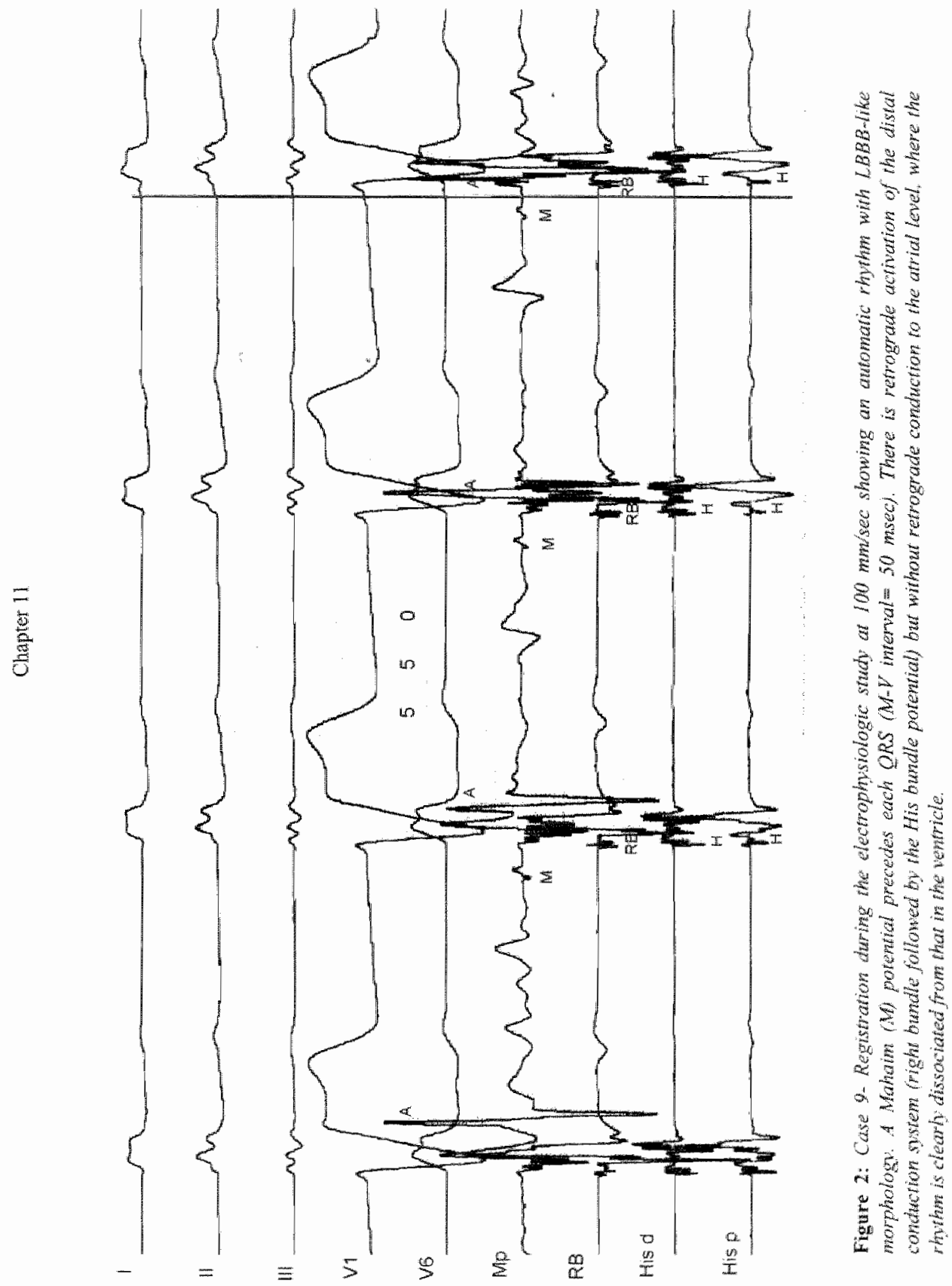




\section{Induction of antidromic CMT by automatic rhythm}

In 2 patients (cases 9 and 33) the automatic rhythm triggered episodes of antidromic tachycardia (figure 4 ). It happened in both cases during isoproterenol infusion. Initiation of antidromic tachycardia was preceded by a long RR cycle due to $A-V$ dissociation caused by automatic beats arising in the Mahaim fiber. The preceding $P$ wave which was not conducted through the $A-V$ node was conducted with a critical slowing in the Mahaim fiber (figure 4). Isoproterenol improved retrograde conduction over the $\mathrm{A}-\mathrm{V}$ node enabling antidromic circus movement tachycardia to become sustained, but the mode of tachycardia induction was not related to the $\mathrm{V}-\mathrm{A}$ conduction itself or to slowing of the sinus rate.

\section{Heat Induced Automaticity}

Automaticity during ablation at the proximal insertion of the Mahaim fiber: Mahaim automatic tachycardia (MAT) was induced during radiofrequency catheter ablation in 30 out of 33 patients $(91 \%)$ when catheter ablation was targeted at the proximal Mahaim potential at the tricuspid annulus. MAT usually started promptly after current delivery (usually in less than 5 seconds). MAT duration was variable lasting from 4 beats to almost 2 minutes (figure 5). Its occurrence could not be prevented by atrial pacing during catheter ablation. In 4 patients with prolonged MAT successful ablation was only achieved after complete elimination of that rhythm (figure $5 \mathrm{~B}$ ).

Automaticity during ablation at the distal insertion of the Mahaim fiber: We did not include in these results the 5 patients who were ablated targeting the distal insertion (table 1). They did develop automaticity but because there were slight differences in QRS configuration we could not rule out that the rhythm was arising in the distal part of the right bundle branch or ventricular myocardium.

\section{DISCUSSION}

Early investigators" 3. 4.11,12 suggested that Mallaim fibers could be a complete accessory atrioventricular conduction pathway with variable length containing $A$ $\checkmark$ nodal like tissue. The intracardiac electrophysiologic testing has been the main tool to solve the Mahaim puzzle. Transient accelerated automatic rhythms arising from the Mahaim fiber during radiofrequency catheter ablation ${ }^{6.13}$. 14 have been recognized as one of the features that such fibers share with $A-V$ nodal tissue. Those rlyythms are analogous to the accelerated junctional rythm (AJR) arising during slow A-V nodal pathway ablation in patients with A-V node reentrant tachycardia. AJR is considered to be a sensitive marker ${ }^{15}$ of a successful ablation site. It seems that heat induced automaticity is as common during ablation of Mahaim fibers as during slow A-V nodal pathway ablation. We found it in 30 out of 33 patients $(91 \%)$ when the atrial insertion was targeted. Other authors like Braun et al ${ }^{14}$ reported similar figures $\left(15\right.$ out of 15 cases $-100 \%$ ) while Heald ${ }^{13}$ et al found it in $57 \%$ ( 12 out of 21 patients).

We found in 4 patients that complete termination of heat induced automaticity may be required as an ablation endpoint to achieve long-term success ${ }^{6}$. 


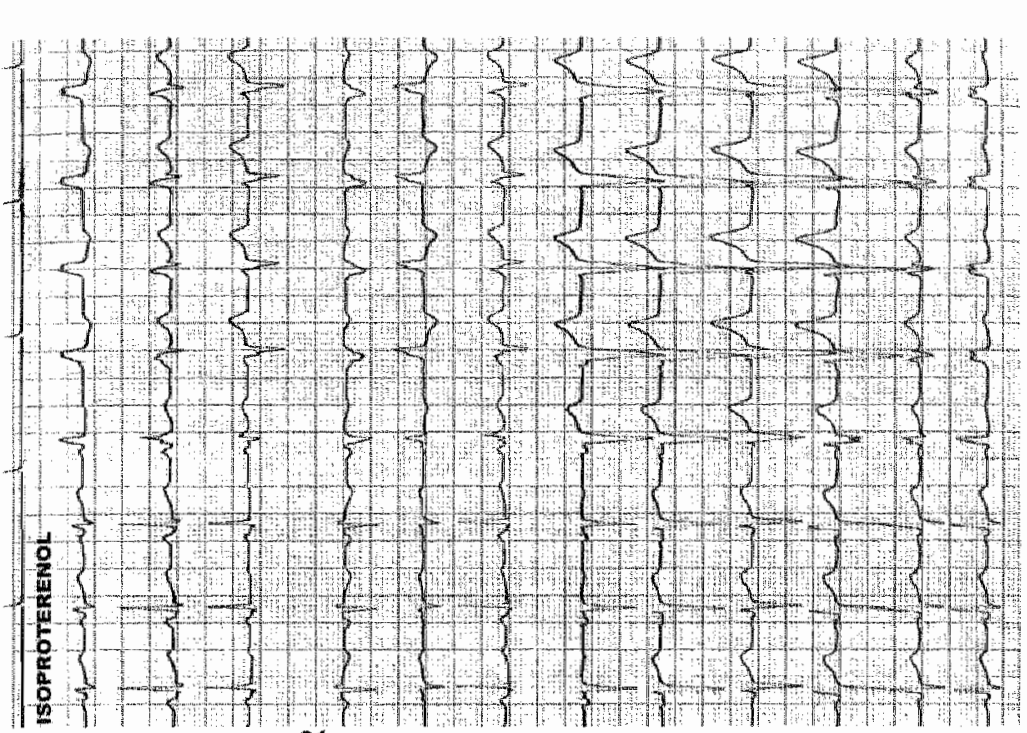

58

장

5 8 要

30

3

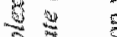

농

2 约

Q

은

$\because 6$

过

8 क

प

ㄴ.

ป ป

5

施

I

동

$=$
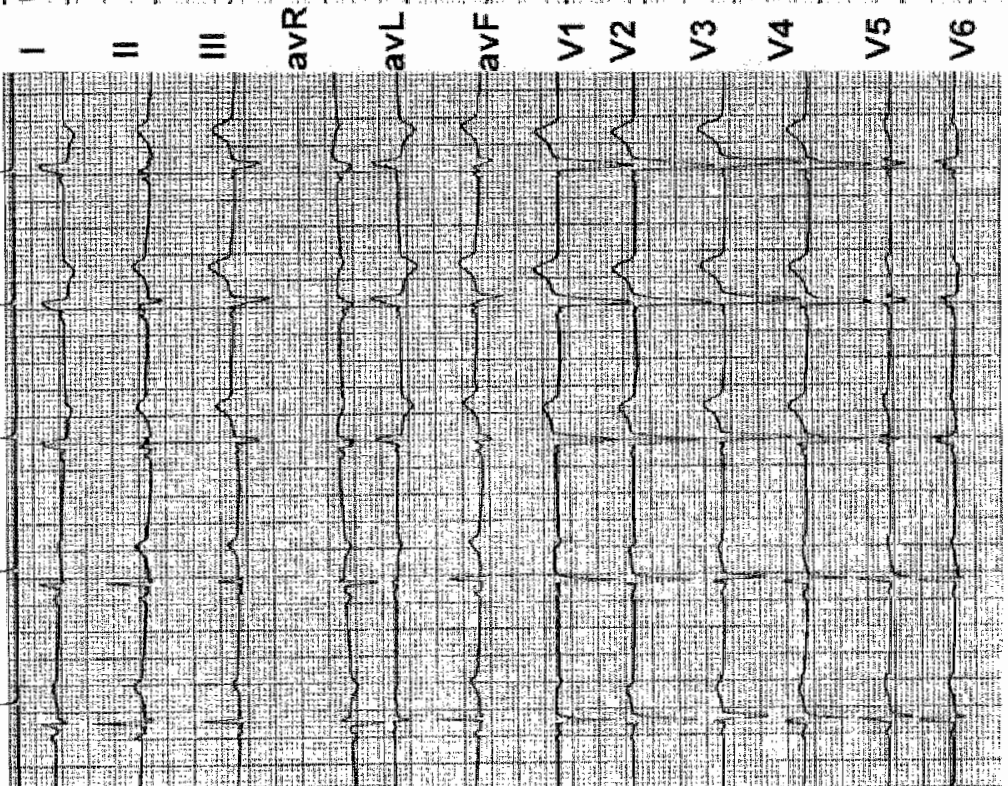

密造

58

52

35 比

ज.

要

施

क ए

0 용

山 8

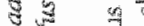

논 9

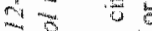

단

然

0 胥

4

의

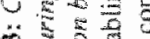

m $\frac{\pi}{3}$

\& क

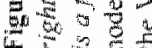




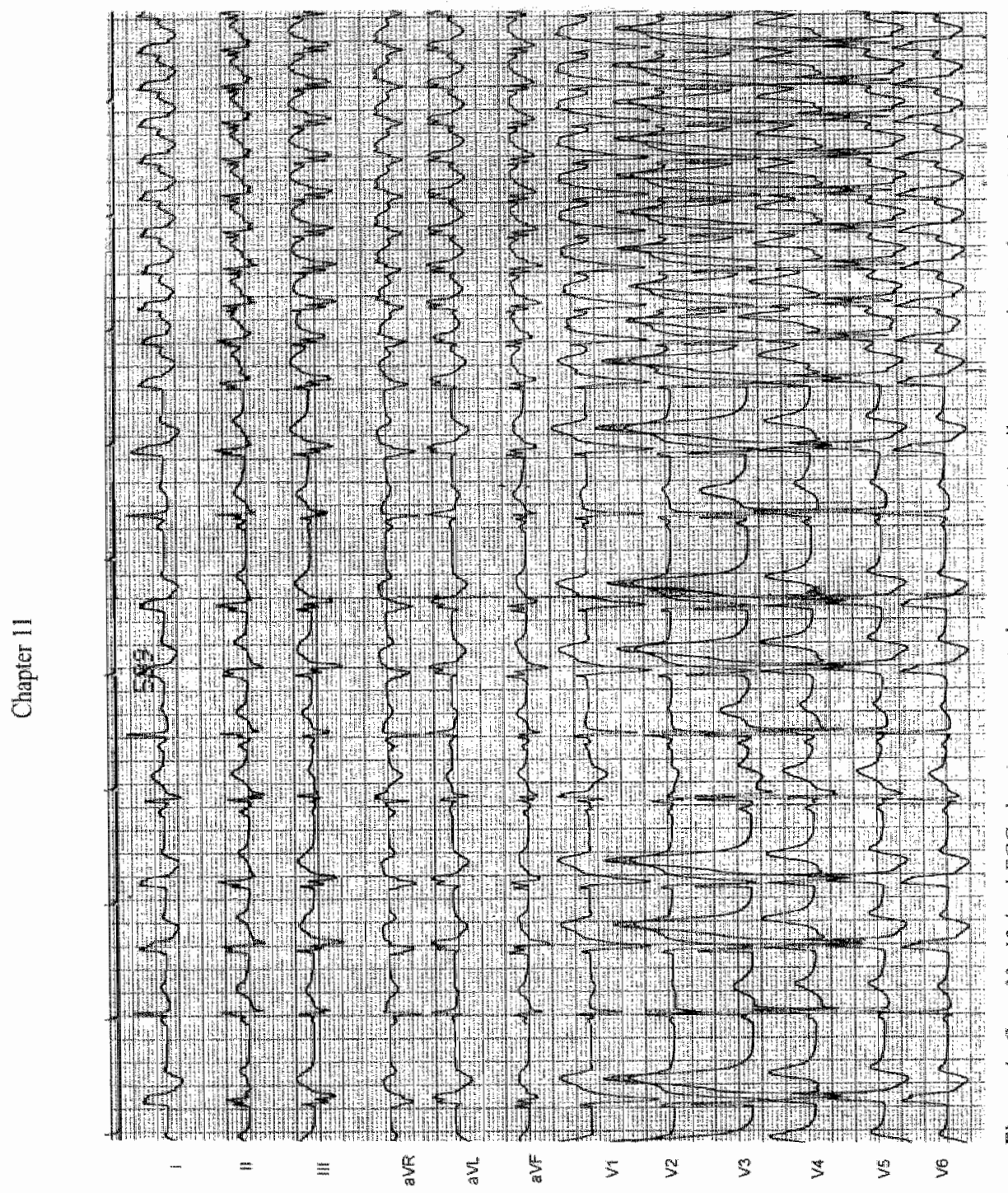

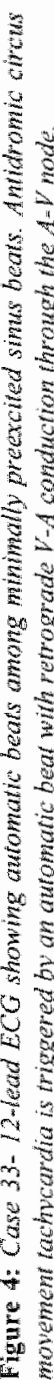




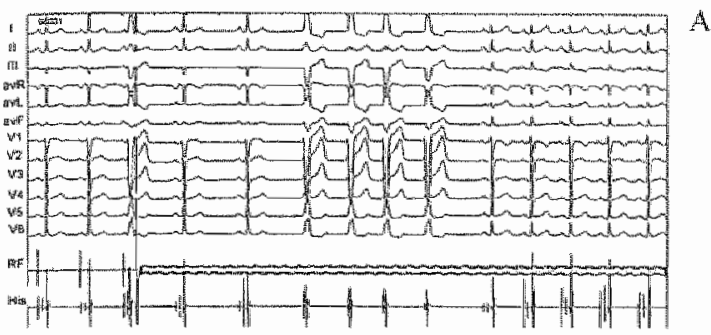

$\mathrm{B}$

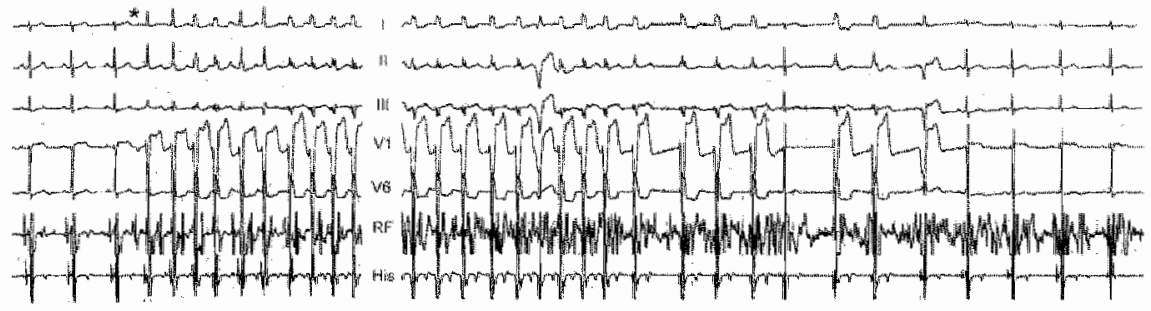

Figure 5: (A) A shori episode of Mahain awtomanicity convining of 4 beats whth LBBB-like

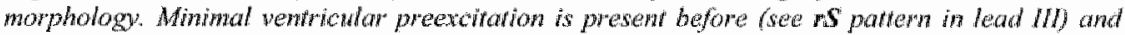

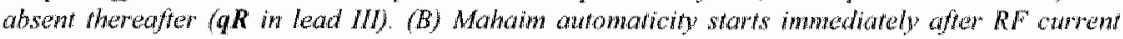

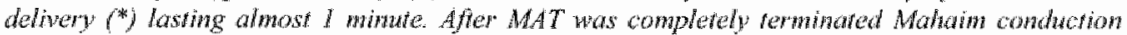
did not recur.

Spontaneous automaticity arising in a Mahaim fiber was first reported by Kanter et al ${ }^{16}$ in a 7 year old child with an incessant bigeminy initially thought to be of ventricular origin. She underwent electrophysiologic evaluation because a 24 hour Holter monitoring showed nonsustained runs suggestive of ventricular tachycardia. During the EP study an atriofascicular pathway was diagnosed. Sosa et al reported a patient with symptomatic runs of an irregular $\angle B B B-l i k e$ tachycardia with $A V$ dissociation, most likely due to spontaneous automaticity. Belhassen et all $^{\text {(ij }}$ reported one patient with a likely automatic escape thythm arising in an atriofascicular pattrway. It was remarkable to find $12,5 \%$ spontaneous automaticity in this large cohort of 40 patients with Mahaim fibers. These young patients presented with a spectrum of automatic rhythms ranging from asymptomatic slow rhythms to fast and repetitive bursts of tachycardia with LBBB-like morphology. This is another shared characteristic with the A-V node ${ }^{17}$. There are a few reports ${ }^{8-20}$ about young patients with AVNRT showing spontaneous accelerated junctional rhythms (A.JR), which can be cured by slow pathway modification with radiofrequency catheter ablation. Epstein at al found in 3 out of 5 patients that AJR served as trigger for AVNRT, parlicularly with AJR at an accelerated rate. We did the same observation in 2 of our 5 patients who had antidromic CMT triggered by automatic beats. He reported an incidence of $17 \%$ (5 out of 29 patients) of spontaneous episodic AJR in a population with a mean age of $12,9 \pm 5,2$ (range 4,2 to 25 ) years, which is similar to our study population with spontaneous automaticity with a mean age of $15 \pm 7(8$ to 26$)$ 
years. We also found that spontancous automaticity was terminated after successful catheter ablation matching Epstein"s group observation that in their patients with AJR and AVNRT it was required to eliminate or drastically modulate the AJR to achieve a long-term successfull result.

The likely mechanism of spontaneous thythms seen in cases 9,33 and 36 was normal automaticity. In those patients Mahaim automaticity was dependent on slowing of the sinus rate ("Mahaim escape rhythm") (figure 3). Isoproterenol infusion increased both sinus and Mahaim automaticity but in those patients a greater increase in the discharge rate was seen at the Mahaim fiber level ("Mahaim active rhythm"). Patients 18 and 22 had an incessant repetitive nonsustained tachycardia which could not be terminated nor induced by programmed electrical stimulation. Isoproterenol infusion in those patients caused only a slight increase in the discharge rate. Those findings suggest an abnormal automaticity as the mechanism of their clinical tachycardia.

\section{CONCLUSION}

Mahaim automaticity occurring during ablation of its atrial insertion is a common event, and its complete termination may be required in some patients to achieve long-term success.

Spontaneous automaticity arising in the Mahaim fiber can serwe as a trigger for antidromic circus movement tachycardia and can be a major clinical problem. It is another characteristic it shares with the electrophysiologic behavior of the $\mathrm{A}-\mathrm{V}$ node.

\section{References}

1. Guiraudon CM, Guiraudon GM, Klein GI: Histologic evidence for an accessory atrioventricular

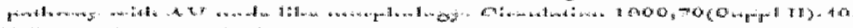

2. Wollens HIJ: Electrical stimulation of the heirt in the study and treatment of tachycardias. University Park Press, Baltimore, 1971

3. Klein GI, Guiraudon GM, Kerr CR, el al: "Nodowentricular" accessory pathway: evidence for a distinet accessory atrioventricular pathway with atrioventricular node-like properties. J Am Coll Cardiol 1988; 11:1035-1040.

4. MeClelland $\mathrm{JH}$. Wang $\mathrm{X}$, Beckman $\mathrm{KJ}$, et al Radiofrequency catheter ablation of right atriofuscicular (Mahaim) aceessory pathways guided by accessory pathway activation potentials. Circulation $1994: 89: 2655-66$.

5. Ellenbogen KA, Rogers R, Old W: Pharmacological characterization of conduction over a Malhain liber: evidence for adenosine sensitive conduction. Pacing Clin Electrophysiol 1989:12:1396*1404.

6. Sternick EB, Gerken LM, Vrandecic MO: Appraisal of "Mahaim" automatic tachycardia. I Cardiovase Electrophysiol 2002:13:244 249.

7. Macle L. Sthah DC. Jais P. Haissaguere M: Accessory pathway automaticity after radiofrequency ablation, 』Cardiovase Electrophysiol 2002:13:285-287.

8. Lerman BB, Josephson ME: Automaticity of the Kent bundle: confirmation by phase 3 and phase 4 block. J Am Coll Cardiod 1985,5:996-999.

9. Sosa E. Scantwacca M: Repotitive, thon-sustaned wide QRS complex tachycardia: what is the tachycardia mechanism? \# Cardiovasc Electrophysiol 2001;12:977-8.

10. Bellaassen B, Ilan $M$, Glick A. Wide QRS thythm in a young woman with recurrent palpitations: what is the diagnosis? I Cardiovase Electrophysiol 2003;14:1376-1378. 


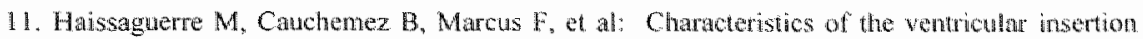
sites of accessory pathways with anterograde decremental condwotion properties. Cinculation $1995 ; 91: 1077 \cdot 1085$.

12. Kuck KH, Siebelss J, Bran E, al: Mahaim fibers - a second atrioventicular gonduction system. Pancing Clin Electrophysiol 1997;20:1201

13. Heald SC Daves W. Ward DE, et al: Radiofrequeney catheter abation of Manaim tachycandia by targeting Malnam potentials at the tricuspid annulus. Brif Hear J1995;73250-257.

14. Braun E, Siobbels I, Volkmer M, et al: Radiofrequency-induced preexcited automatic thython during ablation accessory pathways with Nahain-type prexcination does it predio chimical outcome? Pacing Clin Electrophysiol 1997,20:1121.

15. Thathur RR, Klein GI, Yee R: Juncional tachycardia: a useful marker during radiofequeney

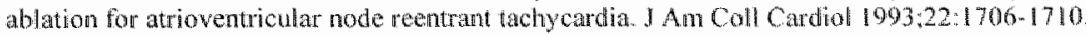

16. Kanter $R$, Saba Z, Garson Jr A: Wide complex bigeminy: musual presentation of an atriofascicular fiber, I Cardiovase Electrophysiol 1994;5:795-802.

17. l. ee PC. Kanter R, Gomez-Marin O, at: Quantitative assessment of the recovery property of atriofascicular/atriowentricular-type Maham fiber. „Catiowase Electrophysiol 2002;13:535. 541 .

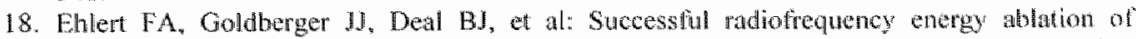
automatic junctional tachycardia preserving nomal atrio ventricular nodal conducion. Pacing Clin Electrophysiol $1993: 16: 54-61$.

19. Lewy AM. Bonazinga B.) Sudden snus slowing with junctional escape: a common mode of initiation of juvenile supraventricular tachycardia. Circulation $1983,67: 84-87$.

20. Epstein MR, Saul IP, Fishberger SB, et al: Sponaneous accelerated functional ahy thm: an unusual but useful observation prior to tadiofrequency catheter ablation for atric vantricular node reentant tachycardia in young patients. Pacing Clin Elactrophysiol 1997,201654m 1601 . 


\section{Chapter 12}

\section{Short atrioventricular Mahaim fibers: observations on their clinical, electrocardiographic and electrophysiologic profile}

Eduardo Back Sternick

Márcio L. Fagundes ${ }^{\dagger}$

Fernando E.S. Cruz Filho ${ }^{\dagger}$

Carl Timmermans $\ddagger$

Eduardo A. Sosa

Luz-Maria Rodriguez $\ddagger$

Luiz M. Gerken*

Mauricio I. Scanavacca ${ }^{d}$

Hein J.J. Wellens $¥$

Biocor Instituto*, Nova Lima, Brazil, Instituto Nacional de Cardiologia Laranjeiras, Ministério da Saúdet, Rio de Janeiro, Brazil, University Hospital ${ }^{\ddagger}$, Maastricht, The Netherlands, Instituto do Coração da Faculdade de Medicina da Universidade de São Paulo, Brazill, University Hospital

Accepted for publication in the Journal of Cardiovascular Electrophysiology 


\begin{abstract}
Introduction: A short atrioventricular decrementally conducting accessory pathway is an uncommon variant of preexcitation. Available data from small series suggests that their decremental properties might not be caused by $A-V$ nodal-like tissue.

Methods: We compared clinical, electrocardiographic and electrophysiologic parameters in two groups of patients: 8 patients with a short A-V Mahaim pathway (Group A), and 33 patients with atrofascicular pathways (Group B). Radiofrequency calheter ablation was carried out guided by activation mapping at the annulus in Group $A$ patients and targeting the " $\mathrm{M}$ " potential in Group $\mathrm{B}$ patients.

Results: After ablation of all associated rapidly conducting bypass tracts, 7 of the 8 Group A patients showed clear preaexcitation. In only 1 of $\&$ patients the short A-V Mahaim fiber was actively engaged in a reentrant tachycardia circuit. During radiofrequency catheter ablation an automatic rhythm occurted in $4 / 8$ patients. Intravenous adenosine caused conduction block in the Mahaim fiber in 3 of 5 patients tested. In group B no patient showed clear prexcitation $(p<0,000 \mathrm{H})$ while $72 \%$ had a minimal preexcitation pattern. Twenty-nine of the 33 patients had a circus movement tachycardia with AV conduction over the atriofascicular fiber. During radiofrequency catheter ablation $30 / 33$ patients showed accessory pathway automaticity. Adenosine caused transient block at the atriofascicular pathway in $11(92 \%)$ of the 12 patients tested. Conclustons: While short decrementally conducting right sided accessory pathways show a typical ECG pattern different from atriofascicular pathways, their ellectrophysiologic properties do not seem to be uniform. Those pathways can be successfully interrupted by catheter ablation.
\end{abstract}




\section{INTRODUCTION}

Decrementally conducting accessory pathways bypassing the tricuspid annulus, and inserting at the antero-apical region of the right ventricle in or close to the right bundle branch have $\mathrm{A}-\mathrm{V}$ node like features ${ }^{1-7}$ Current evidence suggests that they are an accessory conduction system with a proximal $A V$ nodal-like structure and distal branching resembling the His-Purkinje system ${ }^{3.8}$.

However, our understanding of decrementally conducting A-V Mahaim fibers inserting close to the tricuspid annulus is less clear. A better characterization of these fibers is hampered by their rare occurrence. The few reported series ${ }^{9-40}$ are small and show, apart from their decremental properties, no consistent features suggesting the presence of an accessory atrioventricular node. The aim of this study is to describe the electrocardiographic and electrophysiologic characteristics in 8 patients with short Mahaim fibers and to compare these findings with a group of 33 patients with atriofascicular pathways.

\section{METHODS}

\section{Definitions:}

Decrementat conduction property: a cycle length-dependent prolongation of the impulse conduction dime $\geq 30$ milliseconds through the accessory pathway.

Atriofascicular pathway: The His bundle deflection was inscribed after the right bundle deflection during maximal pre-excitation. At maximal ventricular preexcitation, there was a constant QRS-His relationship without further changes from shortening the atrial pacing cycle length. Ventricular activation at the right wentricular apex occurred earlier than at the tricuspid annulus.

Short A-K Mahaim: A decrementally conducting atrioventricular pathway showing ventricular activation at the tricuspid annulus during maximal pre-excitation earlier than at the right ventricular apical region (figure 1 ) was defined by the authors as a "short $A-V$ Mahaim fiber". The His bundle deflection was always unscribed before the right bundle deflection either during sinus rhythm or during atrial pacing with maximal preexcitation (figure 2 ).

\section{Study Population}

We retrospectively studied 47 consecutive patients from 4 Institutions having accessory pathways with long anterograde conduction times and decremental properties during sinus mythm, usually suffering from a tachycardia with anterograde conduction over the decremental pathway, in whom ablation of the accessory pathway was performed. Six patients were ablated targeting the distal insertion of the atriofascicular pathway and were excluded from this study. Every patient included in this series of patients showed a decrementally conducting atrioventricular (A-V) or atriofascicular bypass tract with progressive atrioventricular and AH interval prolongation coupled with a decreasing $H V$ interval leading to a greater degree of ventricular preexcitation with a left bundle branch-like morphology during atrial pacing ${ }^{2}$ (figure 3 ). 


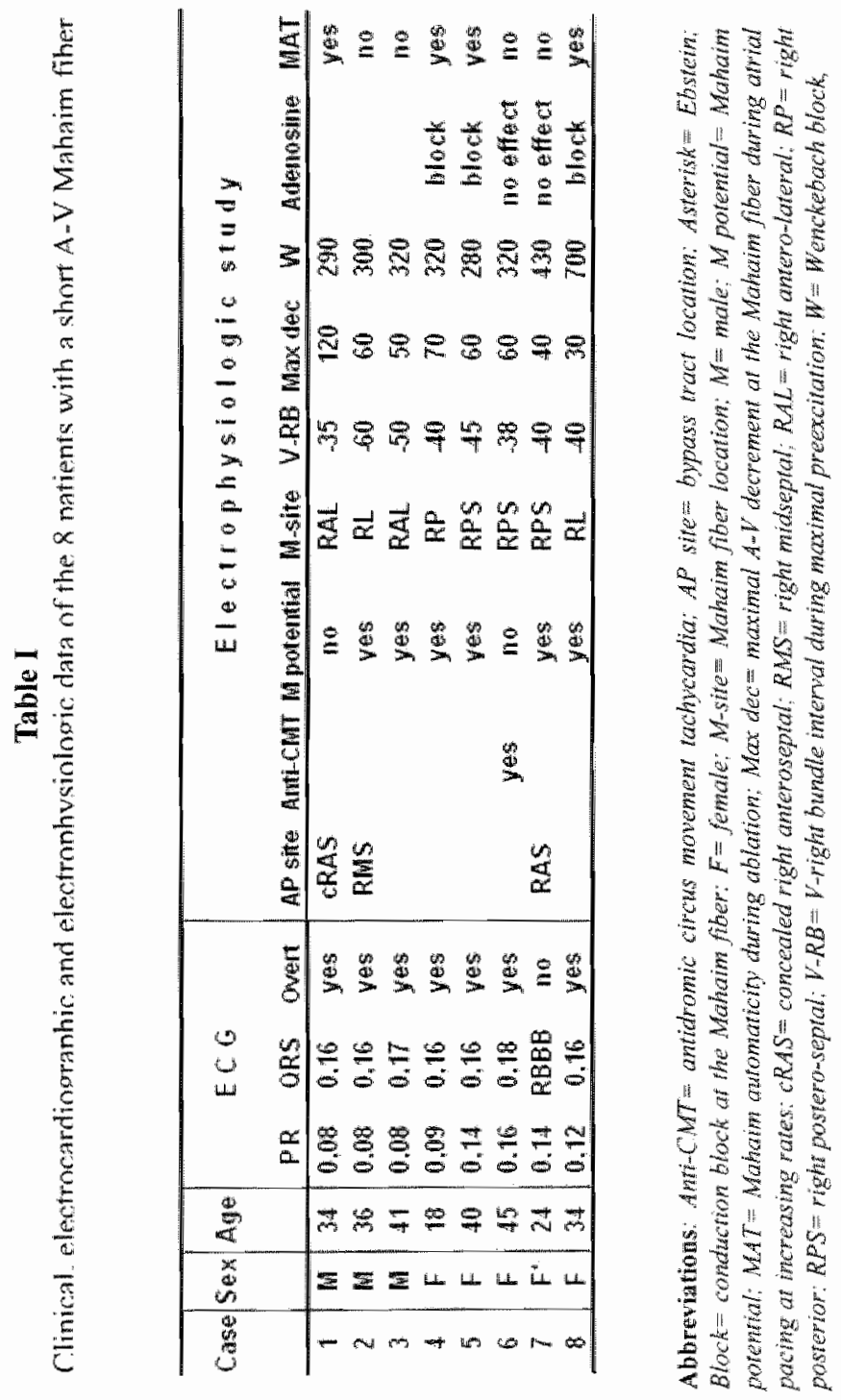




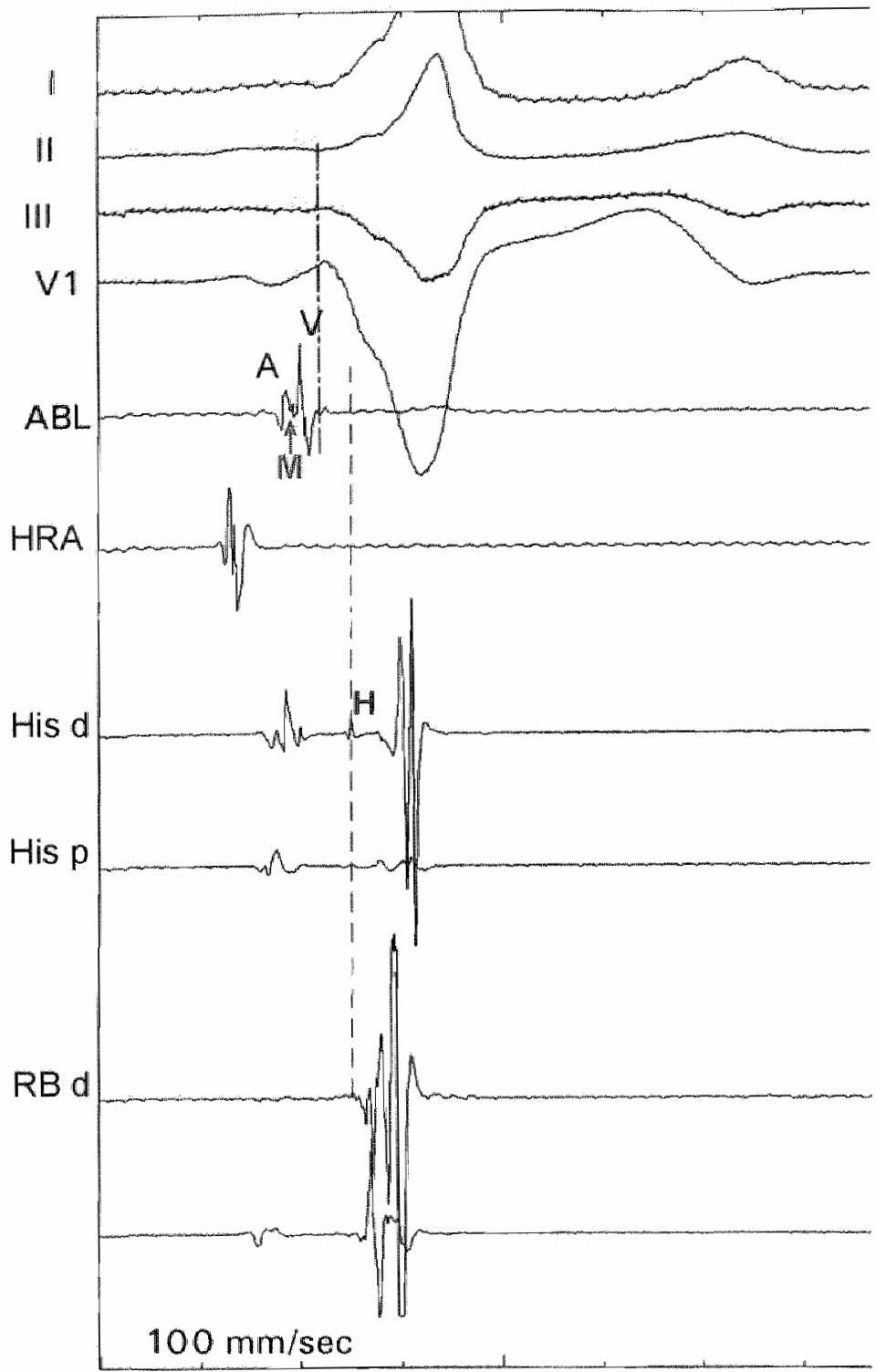

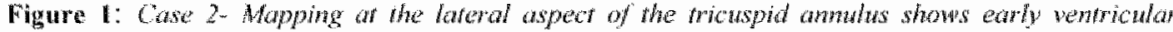

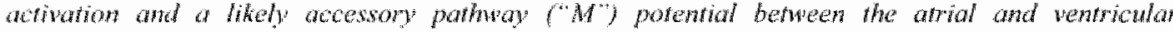

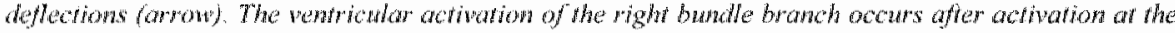
wichspid anmilas. 


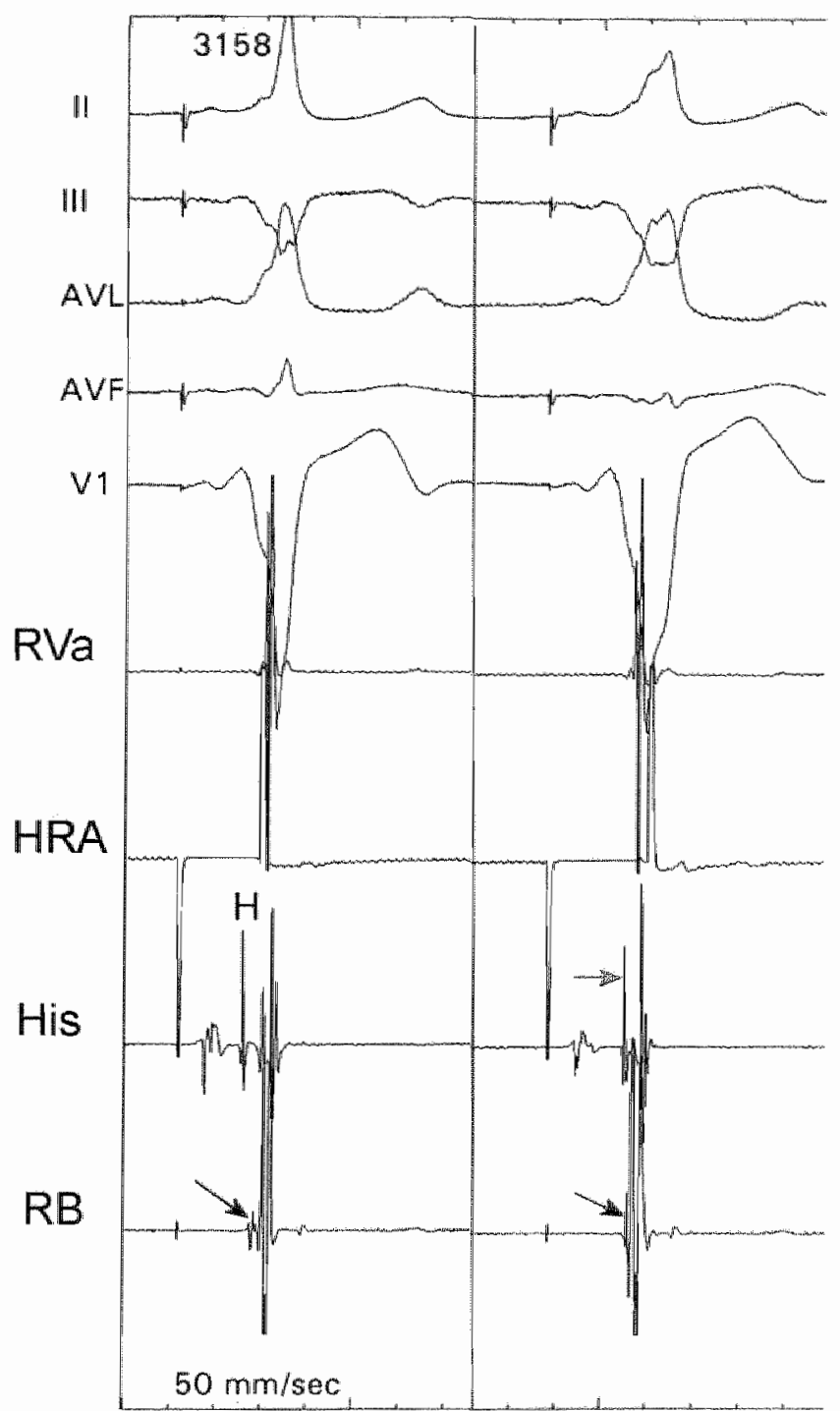

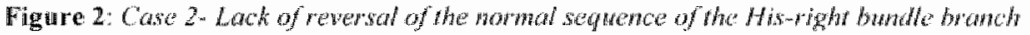
depalariantion daring arrid pacing with imorasing rates.

Group A consisted of 8 patients with a short $A-V$ Mahaim fiber (figure 4). There were 5 females and 3 males. Patients 1 and 2 are brothers. Age ranged from 18 to 45 (mean $34 \pm 8$ ) years. One patient (case 7 ) had Ebstein's discase and 3 patients had an associated rapidly conducting bypass tract (table 1). Four patients had recurrent tachycardia, 3 of them were preexcited and 1 had an orthodromic $A-V$ reentrant tachycardia without bystander conduction over the short A-V Mahaim fiber. Only one tachycardia was related to a short A-V Mahaim fiber. The other 3 tachycardias became non-inducible after ablation of their associated rapidy conducting bypass tracts. Patient 8 had intermittent preexcitation. 
Group $B$ consisted of 20 females and 13 males, with ages ranging from $8-52$ (mean $24 \pm 10$ ) years. All patients were referred for electrophysiologic assessment of a wide QRS tachycardia. Pre-excited. A-V node reentrant tachycardia using an atriofascicular pathway as a bystander was present in $\mathbb{1}$ patient. One patient had atrial fibrillation with preexcited QRS complexes and 2 patients were referred because of repetitive episodes of unsustained tachycardia caused by automaticity arising in the Mahaim frber ${ }^{7}$. Ebstein's disease was diagnosed in $4(12 \%)$ patients.

\section{Adenosine test}

Adenosine triphosphate was administered as a rapid intravenous bolus during atrial pacing. A bolus of $6 \mathrm{mg}$ was followed by another of $12 \mathrm{mg}$ if needed. A non responder was defined as no dellay or block in A-V conduction (prolonged P-delta interval or blocked $P$ waves) in spite of the injection of up to $12 \mathrm{mg}$ of adenosine. Recordings of the 12-lead surface ECG and intracardiac electrogram were made using the EP Tracer or MS System (CardioTek BV, Maastricht, The Netherlands).

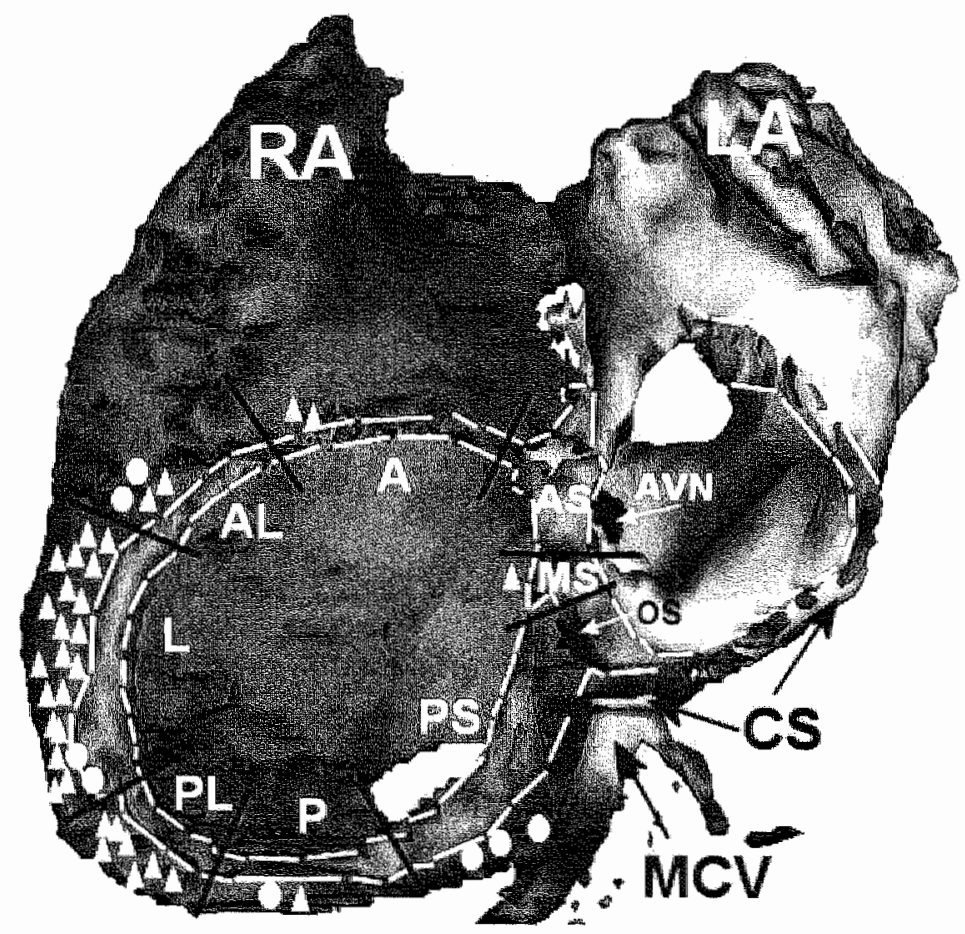

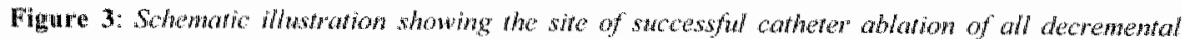

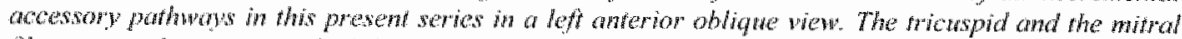

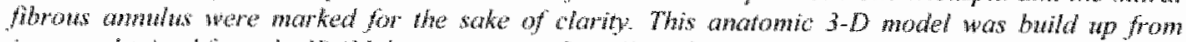

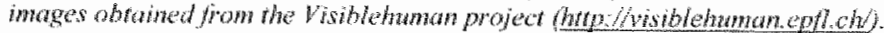

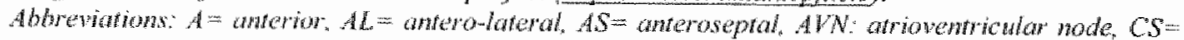

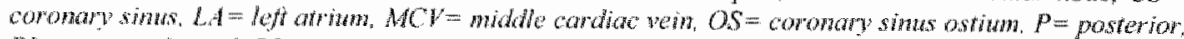

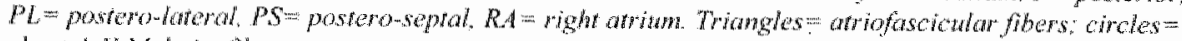
shom A V Maham thers. 


\section{Statistical Analysis}

Values are given as mean \pm standard deviation. The significance of differences ( $P$ $<0.05$ ) between groups of clinical, electrocardiographic or electrophysiologic parameters was assessed by Student's $f$ test or Fisher's exact test.

\section{RESULTS}

\section{Pre-ablation ECG findings}

Group A: All 8 patients had clear ventricular pre-excitation on their baseline 12lead ECG. In six patients ventricular preexcitation occurred because of impulse conduction over a short A-V Mahaim fiber. Two of the 8 patients showed preexcitation due to an associated rapidly conducting $A-V$ bypass tract, one right midseptal and one right anteroseptal. After ablation of those pathways one patient (case 2) showed another preexcitation pattern due to a short Mahaim fiber and patient 7 (with Ebstein's disease) showed no pre-excitation (but only RBBB) during sinus rhythm with preexcitation becoming apparent during atrial pacing (figure 4).

\section{Table II}

Comparative analysis between short (Group A) and long (Group B) Mahaim fibers

\begin{tabular}{|c|c|c|c|}
\hline Variables & Group $A$ & Group B & p value \\
\hline Female gender & $63 \%(5 \%)$ & $60 \%(2033)$ & nis \\
\hline Weall age & $34+8$ & $24 \pm 10$ & 0,02 \\
\hline Minimal preexcitation & $0 \%$ & $72 \%(24)$ & 0.08 \\
\hline Wo preexchitation* & $13 \%(1)$ & $290(9)$ & nis \\
\hline Ovent preexcitation & $98 \%(7)$ & $0 \%$ & $<0,0001$ \\
\hline Associated WPW & $25: 21$ & $15 \%(5)$ & ns \\
\hline Associated CBT & $13 \%(1)$ & $60(2)$ & ns \\
\hline Ebstein & $13 \%|1|$ & $12 * 44$ & ns \\
\hline Mahaim CMT & $13 \%, 1\}$ & $88 \%(29$ & ns \\
\hline Block with adenosine & $3560^{6} h$ & $1112,92 \%$ & ns: \\
\hline M potential & $75 \%(6)$ & $100 \%(33)$ & 115 \\
\hline Mahaim automaticity & $50 \%(4)$ & $91 \%(30)$ & nis \\
\hline
\end{tabular}

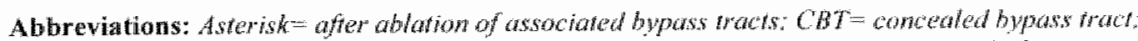

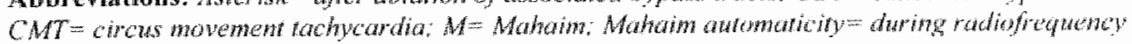
ablaton; ns mon significant p value 
In the seven patients with clear premexcitation during sinus rhythm the P-R interval was nomal in two patients (cases 5 and 6$)$ and short $(<0,12 \mathrm{sec}$.) in 5 patients. As shown in figure 4, the ECG in preexcitation is similar to the ECG in patients with a rapidly conducting right sided accessory pathway.

Group B: Five patients had an associated rapidly conducting accessory pathway. After ablation no patient showed clear pre-excitation. Twenty-four patients had a minimal preexcitation pattern, showing a QRS complex $<0,12$ seconds with an $\mathrm{r}$ pattern in lead III in 20 patients as the most prevalent finding ( 16 of the 20 patients also showed absence of septal $q$ wawes in lead 1$)^{11}$. Two patients had an rsR $^{3}$ in lead III and 2 patients had absence of septal $q$ waves in leads I and V6 as the only $\mathrm{ECG}$ clue for diagnosing minimal preexcitation".

\section{Electrophysiollogic findings}

Group $A$ : During atrial pacing at increasing rates maximal prolongation of the $A$ delta interval ranged from 30 to $120(61 \pm 26) \mathrm{msec}$. Wenckebach block over the short Mahaim fiber (figure 5) occurred with a paced atrial cycle length ranging from 700 to $280 \mathrm{msec}^{\mathrm{D}}$ During maximal preexcitation the mean onset QRS-RBB interval was $43+8$ (25 to 50$)$ msec. No patient showed reversal of His-right bundle potentials during maximal preexcitation. Only one patient (table I) had an antidromic tachycardia with anterograde conduction over the short A-V Mahaim fiber. Three patients had orthodromic tachycardia with their rapidly conducting AP as the retrograde limb of the circuit. None of these patients showed bystander anterograde conduction over the decremental pathway. The short decrementally conducting accessory pathways were mapped by activation mapping and ablated at an anterolateral ( 2 patients), lateral ( 2 patients), posterior (1 patient) and posteroseptal (3 patients) location. An "M" potential, which was not specifically looked for, was recorded in 6 of the 8 patients. The "M" potential can be difficult to assess because it merges with the early local ventricular electrogram. In patient 8 , we were able to document intermittent merging of the "M" potential and the Jocal ventricular electrogram during preexcited beals, because during non-preexcited QRS complex, spontaneous conduction block over the Mahaim fiber occurred in its ventricular insertion, distally to the "M" potential (figure 6). During radiofrequency ablation a Mahain automatic tachycardia occured in 4 of the 8 patients.

In 2 patients the automatic thythm was short-lived and consisted of only 3 beats followed by sinus rhythm without preexcitation (figure 7).

Group B: Patients with atrofascicular pathways showed during atrial pacing Wenckebach block and maximal A-delta prolongation with no significant differences from Group A patients. Mean onset QRS-RBB interval during maximal preexcitation was $-6 \pm 8$ msec $(p<0,0001$ compared with short A-V Mahaim fibers). Thirty of the 33 patients had automaticity during radiofrequency catheter ablation (table 11 ).

\section{Adenosine test}

Five patients in Group $A$ and 12 patients in group $B$ were tested. Three of the five patients $(60 \%)$ and 11 of the 12 patients $(92 \%)$, respectively responded with conduction block in the decrementally conducting accessory pathway (figure 8). 


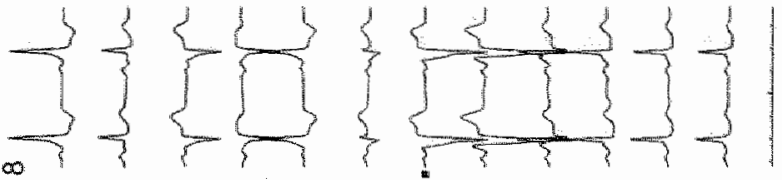

$\frac{3}{3}$

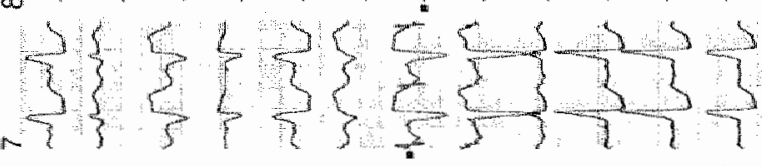
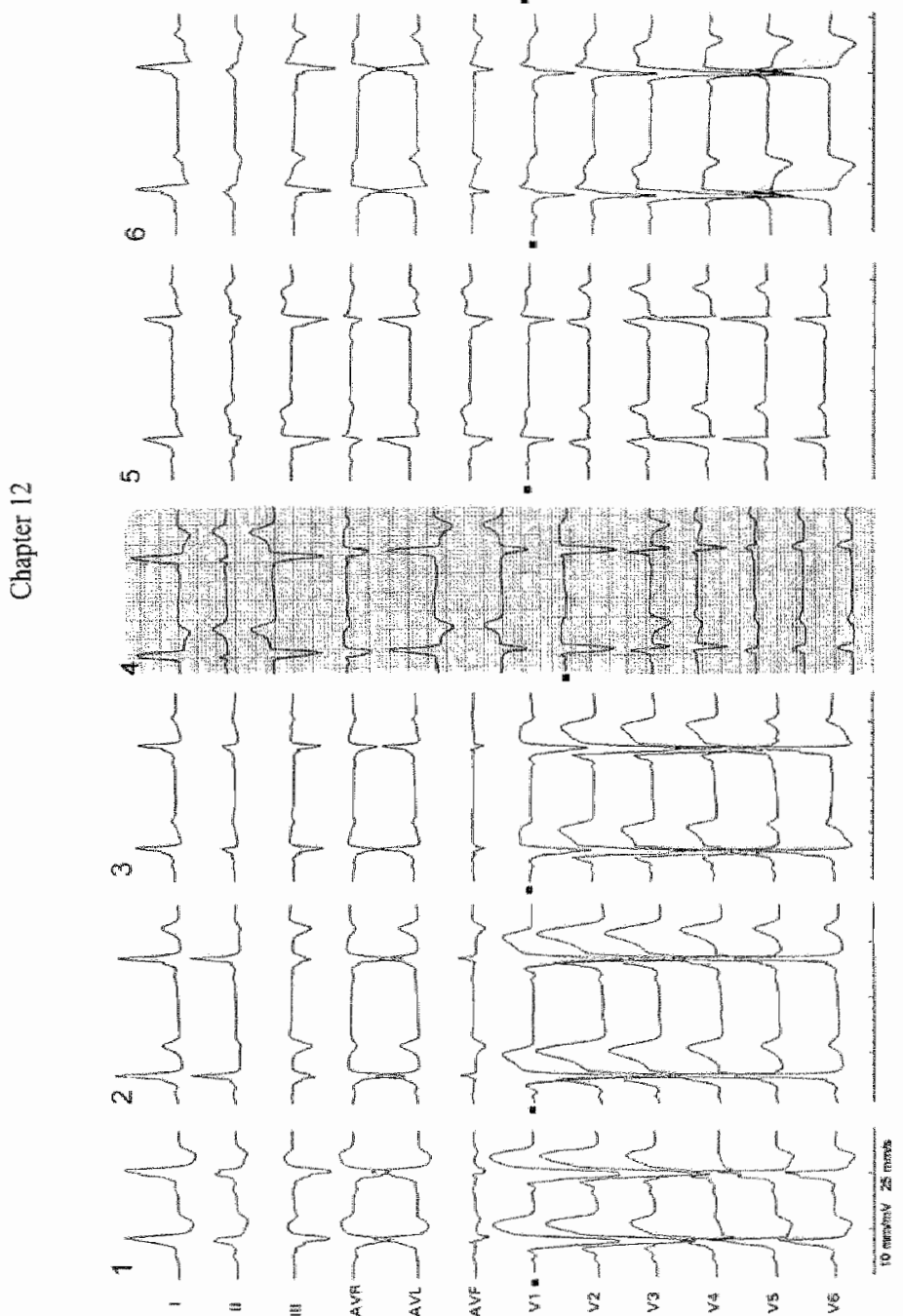

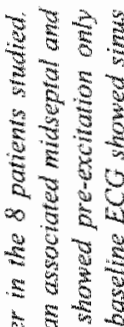

85 क

है

S 5

$\therefore$ 这

क 5

b 4

ह

\&

证

5

50

Un

要业

8 a

है

50 .

है

os -2 站

논

o

4 ह

원

i

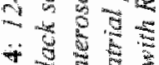

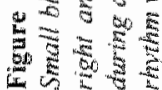




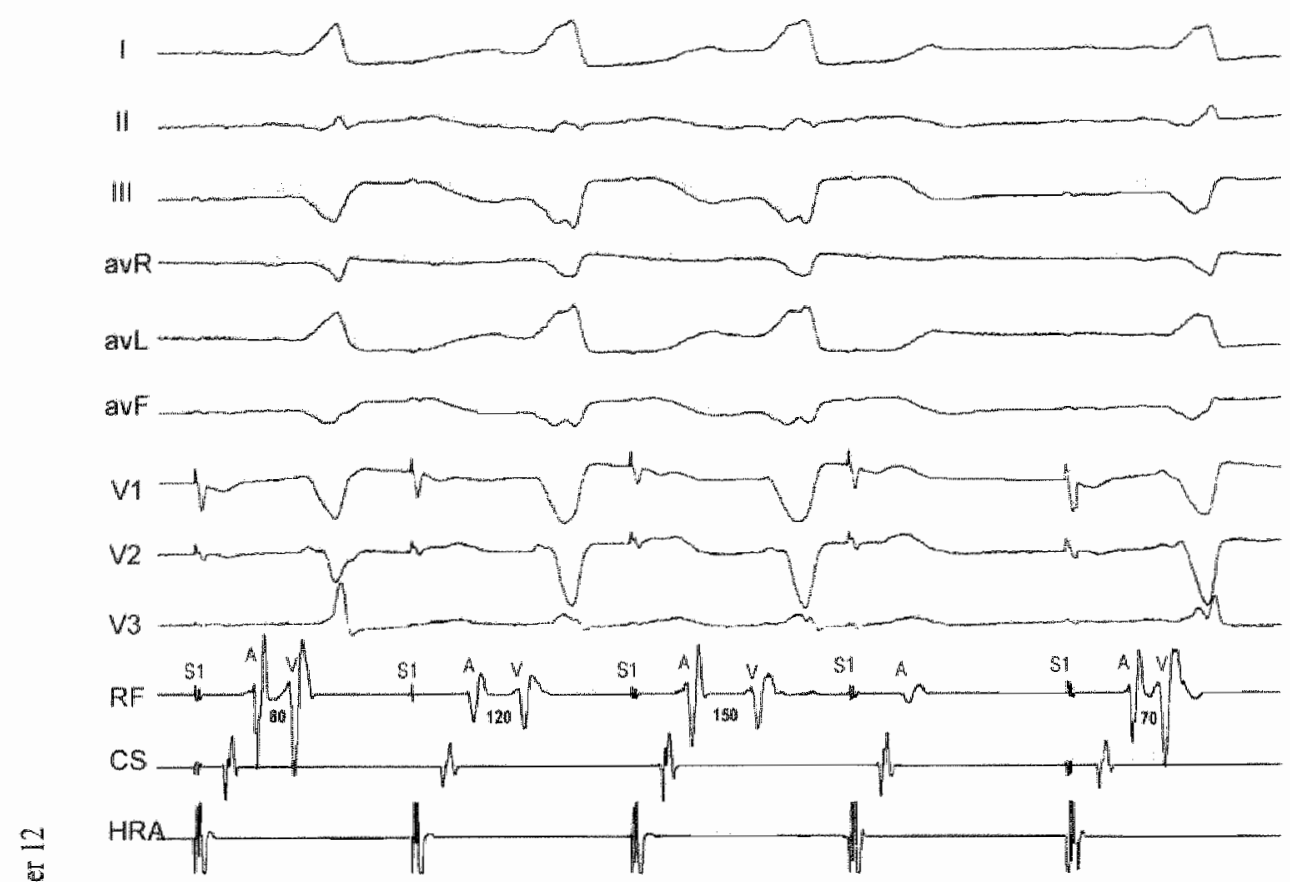

Figure 5: Case 7- Wenckebach block in a short A-W Mahain during right arial pacing al 430 musec.

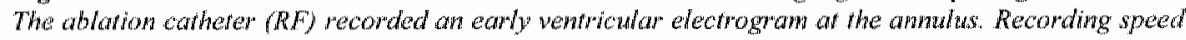
mas $100 \mathrm{~mm} / \mathrm{s}$

\section{DISCUSSION}

\section{Common features in patients with short A-V Mahaüm fibers}

The presence of overt pre-excitation in 7 of the 8 patients can be explained by the large mass of right ventricular myocardium depolarized by the impulse conducted over the short fiber before the arrival of the wavefront proceeding over the HisPurkinje axis. No reversal of the His bundle-right bundle branch activation occurs because when the wavefront over the Mahaim fiber reaches the right bundle branch region, the His bundle and right bundle branch are already activated (fig. 1 and 2). Maximal decremental conduction was not different between Group $A$ and $B$. No patient had retrograde conduction over the Mahaim tiber.

\section{Discordant features in patients with short A-V Mahaim fibers}

The response to an intravenous bolus of adenosine was not uniform in Group $A$ patients. In 3 out of the 5 patients tested, conduction block developed at the Mahaim level. Group B patients showed a different response. Adenosine induced conduction block in the Malaim 

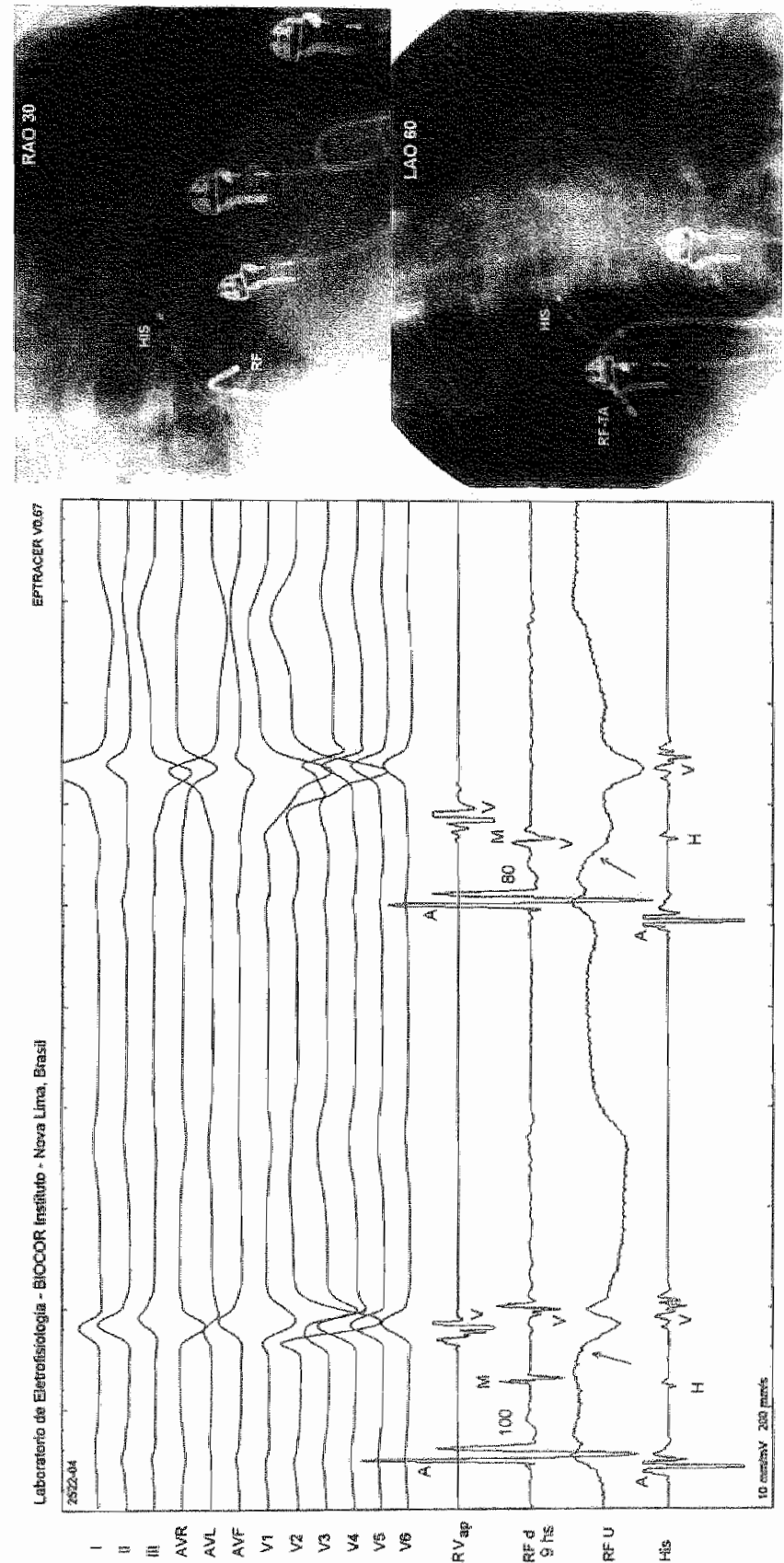

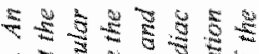

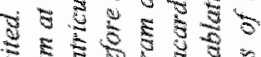

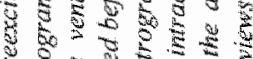

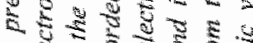

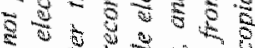
s 5 s.

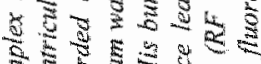

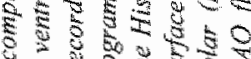

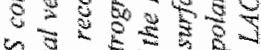

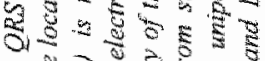
三 है \&

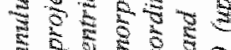
추의

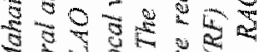

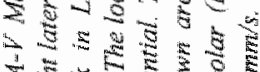

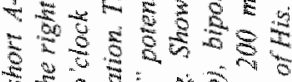

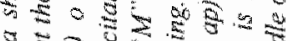

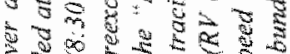

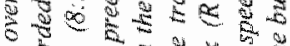
5 5 $5 \div 3$ 의

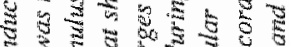

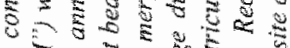
है है है है है है है है 5 क

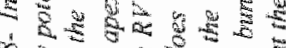
$\infty$ क 35

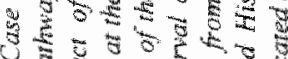

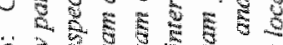

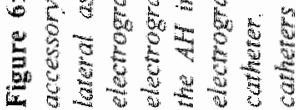

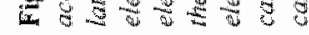




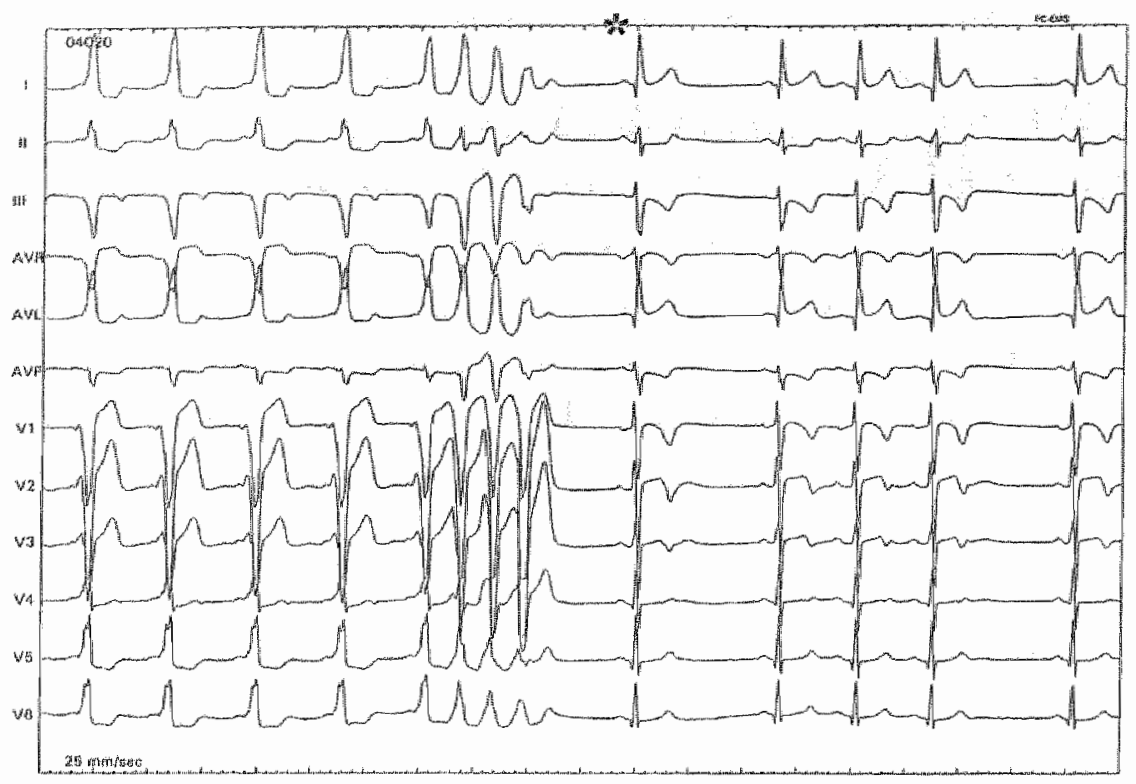

Figure 7: Case 1- A short run of heat induced awomatic whym from the Mahaim fiber vecurring just before block

fiber in 11 of the 12 patients tested. Other authors also found that atriofascicular pathways are sensitive to adenosine ${ }^{8,12}$.

Another difference was the accurrence of theat induced automaticity during radiofrequency catheter ablation. Only 4 group A patients $(50 \%)$ showed ectopic activity arising in the decrementally conducting pathway in contrast to 30 of the 33 patients $(91 \%)$ of Group $B$.

\section{A- $V$ node like features}

Patients 4,5 and 8 responded to adenosine and had automaticity emerging during radiofrequency ablation. Patient 1 showed heat induced automaticity but did not respond to adenosine. The electrophysiologic profile of these patients did not differ from patients with atriofascicular pathways, who also respond to adenosine $(92 \%)$ and show accessory pathway automaticity during ablation (91\%). Braun et $\mathrm{al}^{13}$ reported likewise heat induced automaticity in his 15 patients $(100 \%)$ with atrolascicular pathways. We hypothesize that this subset of patients with short A$\checkmark$ Mahaim fibers who responded to adenosine and showed radiofrequency induced automaticity may have an accessory $\mathrm{A}-\mathrm{V}$ node without a long branching portion as found in patients with atriofascicular pathways. This substrate could be similar to the accessory node-like structure described by Becker et al ${ }^{14}$ at the 
lateral tricuspid valve annulus that connected to the ventricular myocardium beneath the anmulus in a patient with Ebstein's disease and an additional posteroseptal AP.

\section{Short A-V Mahaim fibers without A-V node like behavior}

Patients 6 and 7 did not respond to adenosine nor developed automaticity during RF ablation. Patients 2 and 3 did not have automaticity during ablation but were

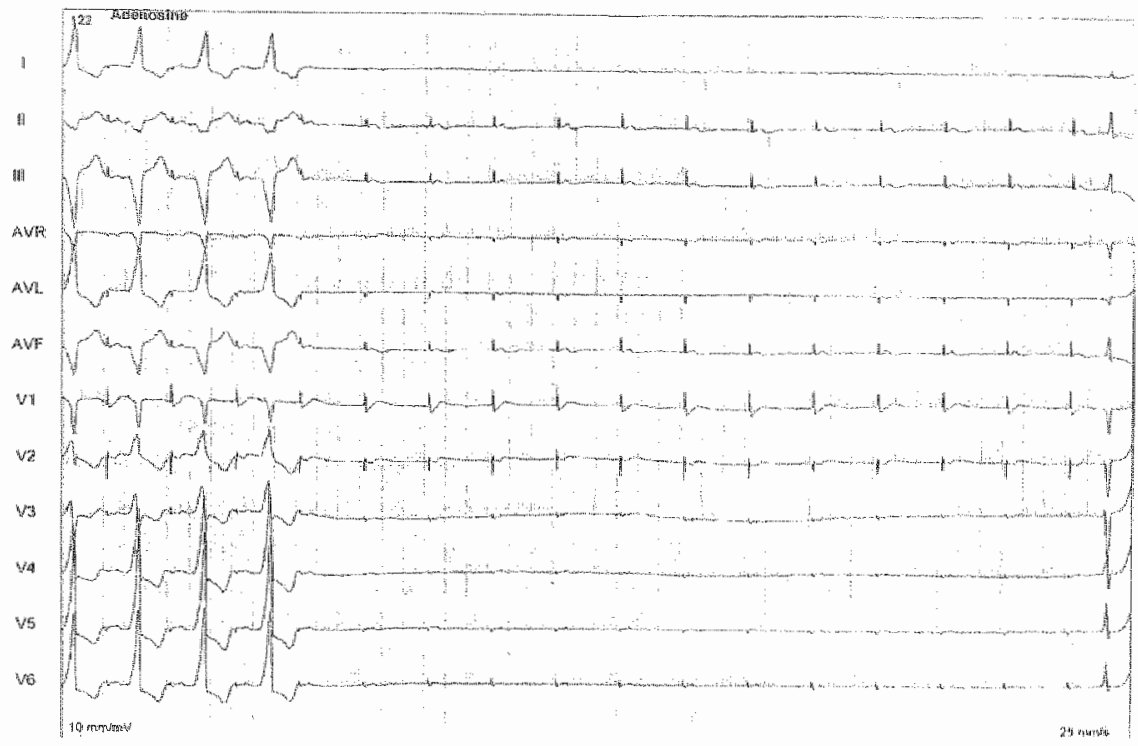

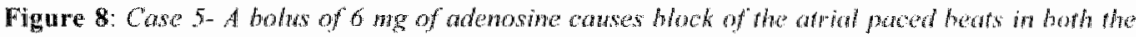

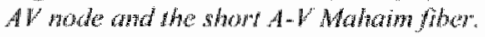

not given adenosine. It is still possible that patients 2 and 3 had an $A-V$ nodal like structure because not all atriofascicular patients show heat induced automaticily. Decremental conduction can occur for reasons other than an A-V node like structure. Haissaguerre ${ }^{10}$ reported a patient who developed decremental conduction after an attempt of radiofrequency catheter ablation over a previous rapidly conducting accessory pathway. Critelli ef al ${ }^{15} .16$ reported one patient with the permanent form of atrioventricular reciprocating tachycardia that developed decremental anterograde conduction over a posteroseptal accessory pathway after A-V node ablation. Histologic examination showed no $\mathrm{A}-\mathrm{V}$ node like-tissue but an accessory pathway with a serpiginous course.

Do all short A-V Mahaim fibers need to undertake catheter ablation therapy? Only one patient in group A had a short A. $V$ Mahaim incorporated in a reentrant tachycardia circuit as compared with 29 from 33 patients with atriofascicular pathways $(p=0.09)$. In addition, 2 patients have a very long effective refractory 
period. Those patients without tachycardia and with a very long effective refractory period probably do not need an ablative therapy.

Previous studies: There are very few data on "whort A-V Mahaim fibers" from the literature. Heald at al reported 4 cases with short $A-W$ Mahaim fibers in a series of 21 patients with decrementally conducting accessory pathways. All 4 patients showed overt preexcitation and were ablated at the tricuspid annulus. An "M" potential was found in 2 of the 4 patients and they were not challenged with adenosine. Twelve of his 21 patients had automaticity during ablation (they call it "stuttering block"), but there is no mention whether the short A-V Mahaim fibers also showed radiofrequency induced automaticity. Haissaguerre et al ${ }^{10}$ also reported a series with 21 patients with Mahaim fibers and 4 of them were classified as short A-V Mahaim fibers, all of whom were preexcited during sinus rhythm, as well. Two of them (50\%) were tested with adenosine and did not respond. They did not mention the occurrence of Mahaim automaticity during catheter ablation. Cappato et al ${ }^{17}$ had 1 short $A_{-}-V$ Mahaim in their series of 11 patients. He found an accessory pathway potential but there is no information regarding adenosine test or automaticity during ablation.

\section{Limitations of the study}

There are some limiting factors to our study as the small number of patients challenged with adenosine. Our assumption of defining the group A patients as. having a "short A-V Mahaim fiber" is not based in any histologic data. Howewer, there is sound electrophysiologic evidence derived from local ventricular activation mapping that supports our working definition.

\section{CONCLUSION}

Short decrementally conducting right sided accessory pathways show a typical ECG pattem different from atriofascicular pathways. However, their electrophysiologic properties may not be uniform. These pathways can be successfully interrupted by catheter ablation.

\section{References}

1. Mahaim I. Bennet A: Nouvelle recherches sur les connewions superieures de la branche gatuche du raiscea de His Tawara ave cloison interventriculate. Cudiologia 1938;1:61-76.

2. Wellens H.I: Electrical stimulation of the heart in the study and treatment of tachycardias. University Pak Press. Ballimoke $\| 971$.

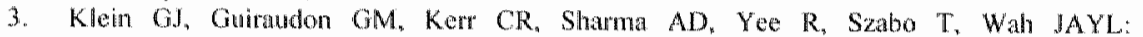
"Nodovantricular" acessory pathway: evidence for a distinct accessory atroventricular pathwy with attoventricular nodeme properties. I An Coll Cardiol 1988;11:1035-1040.

4. Guiraudon CM. Gutraton GM, K lein GJ; Histologic evidence for ars acessory atrioventricular pathway with AV-nodemlike morphology. Circulation 1988; 78 (Suppl II): 40.

5. Ellenbogen KA. Ranura NM, Packer DL, O'Callagan WG, Greer GS, Sintetos AL, Gibert MR. German LD: Accessory nodoventricular (Mahaim) fibers. A clintical review : Pacing Clin Electrophysiol 1986:9:868-884.

6. Sternick EB. Gerken LM. Vandecic MO: Appraisal of" "Mahaim" automatic tachycardia: I Cardiovase Electrophysiol $2002 ; 13: 244-249$. 
7. Sternick EB, Sosa EA, Timmermans C, Cruz FE, Rodrignez LM, Gerken LM, Scanawaeca MI, Fagundes $\mathrm{ML}$, Bueno $\mathrm{SC}$, Vrandecic $\mathrm{MO}$, Wellens H.J. Automaticity in Mahaim fibers, ) Cardiovase Electrophysiol 2004;15:738-744.

8. MoClelland M, Wang X, Beckman KJ. Hazlitu HA. Prior MI, Nakagawa H, Lazzara R, Jackman WM: Radiofrequency catheter ablation of right atrofascicular (Mahaim) aeessory pathways guided by accessory pathway activation potentials. Circulation 1994:89:2655-66.

9. Heald SC, Davies DW, Ward DE, Garrat CJ, Rowland E: Radiofrequency catheter ablation of Mahaim tachycardia by targeting Malwaim potenhials at the tricuspid annulus. Br Heart J 1995;73:250-257.

10. Haissaguere M, Cauchemez B. Marcus P, Le Metayer P, Lauribe P, Poquel F, Gencel L. Clémenty 1: Characteristics of the ventricular insertion sites of accossory pathways with anterograde decremental conduction properties. Circulation 1995;91:1077-1085.

11. Stermick EB, Timmermans C. Sosa EA, Cruz FE, Rodrigucz LM, Fagundes M, Gerken LM, Wellens HJJ: The electrocardiogram in sinus thythm and tachycardia in patients with Mahaim libers. The importance of an "rS" pattern in lead 111. I Am Coll Cardiol 2004:44:126-135.

12. Ellenbogen KA, Rogers R, Old W. Pharmacological characterization of conduction over a Mahaim fiber: evidence for adenosine sensitive conduction. Pacing Clin Electrophysiol 1989;12:1396-1404.

13. Braun E, Siebbels J, Volkmer M, Ouyang F, Hebe J, Willens $S_{n}$ Cappato R, Kuck KH. Radiofrequency-induced preexcited automatic rhythm during ablation accessory pathways with Mahaim-type preexcitation: does it predict clinical outcone? Pacing Clin Electroplysiol 1997;20:1121.

14. Becker AE, Anderson RH, Durrer D, Wellens HJJ; "The anatomical substrates of WolftParkinson-White syndrome; a clinicopathologic contation in seven patients. Am J Cardiol $1981 ; 48: 47-58$.

15. Critell $G$, Perticone F. Coltorti F, Monda $V$, Gallagher J: Antegrade slow by pass conduction after closed-chest ablation of the His bundle in permanent junctional reciprocatiog tachycardia. Circulation 1983;67:687-692.

16. Critelli $G$, Gallagher IJ, Monda V, Coltorti F. Scherillo M, Rossi L: Anatomic and electrophysiologic substrate of the permanent form of junctional reciprocating lachycardia. 1 Am Coll Cardiol 1984;4:601-610.

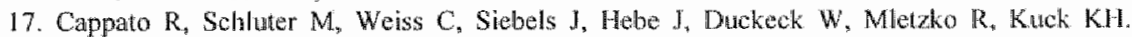
Catheter-induced mecharnical conduction block of right sided accessory fibers with Mahaimtype preexcitation to guide radiofrequency ablation. Circulation 1994:90:282-290. 


\section{Chapter

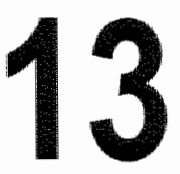

\section{Summary and Conclusions}

In Chapter 1 we present the history of the most important published data on the issue of variants of pre-excitation, highlighting diagnostic aspects and therapy.

According to Webster's New Dictionary the term variant means a manifesting variety, something varying "slightly" from the standard form. The so-called Mahaim fibers are connecting structures with a great variability in their proximal and/ or distal insertions, but sharing a common electrophysiological behavior suggestive of an AV nodallike structure. The term variant is more appropriately applied for their electrophysiologic profile than their anatomic structure. The main source of the concoptual mismatch between anatomy and function of those structures derives from the fact that Ivan Mahaim originally described comections between the A-V node and bundle branches or ventricular myocardium, which bypassed the His bundle, in pathologic specimens. When other authors observed accessory bypass tracts with long and decremental conduction, they assumed that the substrate was the fibers described by Mahaim.

Two of the original structures described by Mahaim, the nodoventricular and nodofascicular fibers are rare items as shown by rare case reports. The majority of patients with anterogadely decrementally conducting accessory pathways are today classified as having either an atriofascicular pathway or a long atrioventricular pathway with decremental properties. Those patients usually show a pre-excited left bundle branch block shaped QRS during tachycardia.

Our aim in Chapter 2 was to describe the differential diagnosis of a left bundle branch block tachycardia with emphasis on the electrocardiographic features which can be helpful for the diagnosis of an SVT with $\angle B B B$, a preexcited tachycardia due to a rapidy conducting right-sided bypass tract, a preexcited tachycardia due to a slow conducting right-sided accessory pathway, and a ventricular tachycardia. We also discussed ECG characteristics suggesting specific mechanisms of ventricular tachycardia like ischemic scar VT, bundle branch and interfascicular reentrant VT, right-sided idiopathic VT and VT associated with right ventricular dysplasia.

The electrocardiogram of patients with atriofascicular pathways are usually described as nomal. A minimally pre-excited ECG is also recognized by some authors in spite 
of the lack of any specific feature for a Maham fiber. We analyzed a large series of patients with Mahaim fibers and in Chapter 3 where we describe our observations regarding the $\mathrm{ECG}$ characteristics in sinus rhythm and during tachycardia in a cohort of 40 patients. The manuscript focuses on 33 patients, because for the sake of clarity we have excluded 5 parients with associated rapidly conducting bypass tracts and 2 patients with short atrioventricular pathways with long and decremental conduction.

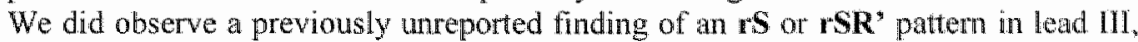
usually associated with the absence of septal $q$ wave in the left-sided leads in a significant $70 \%$ of the patients. An rS pattern in lead III also emerged in 4 out of the 5 patients with WPW and Mahaim after successful ablation of their rapidly conducting accessory pathways. There was a positive relation between the presence of this pattern and left axis deviation during pre-excited tachycardia. This fact together with the observation that after successful ablation of the Mahaim tiber a different QRS morphology emerged in lead III (usually a qR or QR) validated the concept that the $\mathrm{rS}$ pattern represented an electrocardiographic clue as to the presence of minimal right ventricular pre-excitation.

Upon our experience with this large series of Mahain patients we described in Chapter 4 , in a systematic way, the different tachyartyhmias associated with the Mahaim fibers. Every variety was described and some clues for thit ECG recognition are presented.

In Chapter 5 we have tested the sensitivity, specificity and predictive value of previously published electrocardiographic criteria for diagnosing Mahaim tachycardia in 40 patients. The predictive value was evaluated by having a subset of 35 patients with LBBB SVT due to orthodromic A-V tachycardia, A-V node reentrant tachycardia and atrial tachycardia. The population with the lowest predictive value was the one having $\mathrm{LBBB}$ SVT during circus movement tachycardia with V-A conduction over an accessory A-V pathway with a $26 \%$ (5 of 19 patients) false positive diagnosis for Mahaim tachycardia (negative predictive value of $82.5 \%$ ). Previously reported criteria were sensitive only for atriofascicular pathway $(87,5 \%)$ but not for decrementally conducting atrioventricular accessory pathway $(0 \%)(\mathrm{p}<$ 0,00011 ).

We analyzed in Chaprer 6 the electrocardiogram during sinus rhythm of 7 patients with a fasciculoventricular pathway and compared them with the ECG of 20 patients with an antero-septal accessory pathway and with 20 patients with a mid-septal accessory pathway. The ECG of a fasciculoventricular pathway showed a narrower $(0.12 \pm 0.02)$ QRS complex as compared with other septally located bypass $\operatorname{tracts}(0.14 \pm 0.01)(\mathrm{p}<0.0001)$. According to our findings fasciculoventricular pathways have overlapping electrocardiographic features with both anteroseptal and midseptal accessory bypass tracts, therefore they can not be differentiated by any criteria from the antero-septal nor the mid-septal bypass tracts when a larger ORS complex is present, calling for an electrophysiologic study for a correct diagnosis.

In Chapter 7 we report for the first time the engagement of a Mahaim fiber (atriofascicular pathway) in a non-reentrant tachycardia with a $1: 2 \mathrm{P}$ to QRS relationship. The presence of wo connections between the atrium and the ventricle is the usual substrate underlying a tachycardia with 1:2 P to QRS relationship. In this particular case there was only one pathway involved. The pathophysiology was 
related 10 longitudinal dissociation of the impulse conduction within the Mahaim fiber. Duality of conduction over Mahaim fibers due to longitudinal dissociation was reported as an explanation to cycle length alternans during antidromic tachycardia, but it was never before linked to $1: 2$ conduction.

The fasciculoventricular pathways are extensively discussed in Chapter 8 . In spite of the lack of a role in reentrant arhythmias, we emphasized the importance of a correct differential diagnosis between such pathways and a right anteroseptal parahissian bypass tract to avoid damage to the $A-V$ node if an ablation attempt is mistakenly carried out. This possibility is increased by the high incidence of associated rapidly conducting bypass tract along with the fasciculoventricular pathways (we have reported a patient with 2 associated bypass tracts). The electrocardiographic and electrophysiologic characteristics are presented and discussed, as well as a review of the limited literature on the subject.

We did appraise the role of radiofrequency induced automaticity over Mahaim fibers in Chapter 9. The phenomenon had been widely reported but not systematically studied. We described the ECG characteristics of what we have called Mahaim automatic tachycardia ( MAT), its relation to the thermal injury to the proximal part of the Mahaim fiber, its resemblance with the automatic junctional tachycardia occurring during the slow AV nodal pathway ablation, their importance as a hallmark of successful ablation and we suggest for the first time that in some patients it might be necessary to continue the delivery of radiofrequency current until all automaticity arising in decremental pathway is completely terminated.

Chapter 10 is a case study describing our findings in a patient with a Mahaim fiber who underwent radiofrequency catheter ablation at its distal insention site. Our findings were consistent with a long atrioventricular Mahaim fiber inserting in the musculature of the anteroapical area of the right ventricle and not at the right bundle branch as it would be the case of an atriofascicular pathway.

Our aim in Chapter 11 was to describe the occurrence of spontaneous automaticity arising in Mahaim tibers, which included different phenomena ranging from heat induced automaticity occurring during catheter ablation, spontaneous asymptomatic slow automatic thythms, spontaneous automatic thythms that could trigger a preexcited tachycardia under enhanced adrenergic tonus and spontaneous symptomatic fast automatic rhythms presenting as repetitive tachycardia with an incessant character. We discussed those automatic arrhythmias making an analogy with similar spontaneous automatic rhythms arising in the $\mathrm{A}-\mathrm{V}$ node. We conclude that these lindings are consistent with the A.V node like structure of the Mahaim fiber.

In Chapter 12 we analyzed a group of 8 patients with a short A-V Mahaim fiber and compared their clinical, electrocardiographic and electrophysiologic characteristics with a group of 33 patients with an atriofascicular pathway or a long decrementally conducting atrioventricular pathway. Patients with a long Mahaim fiber showed a very homogeneous electrophysiologic profile. A different situation was found in patients with a short A-V Mahaim fibers. They could be classified into 2 different groups: one group comprised of patients with fibers having A-V node like properties and another group without those properties. 


\section{GENERAL CONCLUSIONS}

1. The so-called "Mahaim variants" bypass tracts are rare structures with different anatomic substrates sharing the common electrophysiologic feature of unidirectional slow and decremental anterograde conduction with a variable pathophysiology: because of intrinsic $A-V$ node like properties of the fiber, ordinary muscular fibers showing slow conduction due to anisotropic conduction or because of scar due to previous ablation attempts, or because of a proximal connection to the A-V node or His-Purkinje system. As previously reported, we present additional evidence suggesting that some Mahaim fibers do not insert into the right bundle branch.

2. They can occur as a single pathway or be associated with rapidly conducting accessory pathways. They are involved in many different cardiac arthythmias, sometimes with complex circuits. A non-reentrant tachycardia caused by a $1: 2 \mathrm{P}$ to QRS relationship is reported for the first time. Only the fasciculoventricular variety of Mahaim fiber does not participate in reentrant circuits.

3. The ECG of a patient with a short A-V Mahaim fiber is indistinguishable from the ECG of a rapidly conducting right sided accessory pathway, except when it is associated with a normal PR interval $(29 \%)$. Electrocardiographic recognition of the Mahaim fiber during sinus rhythm is possible in up to $70 \%$ of the patients. Their peculiar minimal pre-excitation ( $\mathrm{rS}$ ) pattern is best seen in lead III, and that observation when matched with the absence of a $q$ wave in lead $\mathbb{\|}$, seems to be very specific of a Mahaim fiber. Tachycardias with anterograde conduction over a Mahaim frber usually can be suspected by the presence of an $\mathbf{R}$ wave in lead $\mathbb{l}$, an IS pattern in lead V1, QRS frontal plane axis between 0 and -75 , QRS transition in the precordial leads after V4 and a QRS width below 0,15 seconds. The negative predictive value of those criteria was $82,5 \%$ and the sensitivity $87 \%$ for atriofascicular pathways. They are not helpful for identilying tachycardia associated with atrioventricular Mahaim fibers.

4. Spontaneous and radiofrequency induced automaticity arising in atriofascicular pathways as well as with short and long decrementally conducting arrioventriculatr pathways tre characteristic features pointing to an accessory $A-V$ node like structure. 


\section{Sumário e Conclusões}

No Capítulo 1 apresentamos um laistórico da evoluçăio do conhecimento acumulado nas últimas 3 décadas na literatura, após análise dos trabalhos mais relevantes sobre variantes de pré-excitaçăo, enfatizando os aspectos diagnósticos e terapeuticos.

De acordo com o dicionário Webster o termo variante significa uma manifestação de variedade, algo que difere levemente da forma habitual. As fibras de Mahaim são estruturas de conexão com uma grande variação nas suas inserções proximais e distais, mas tendo em conum um padräo de resposta eletrofisiológica que sugere fortemente uma estrutura semelhante ao nó atrioventricular ( $\mathrm{A}-\mathrm{V})$. O termo variante é apropriado a estas estruturas mais pelo seu perfil eletrofisiológico, pois, do que pela sua anatomia. A fonte principal para esta dicotomia entre anatomia e função destas estruturas advén do fato de que elas foram originalmente descritas por Ivan Mahairn como conexões entre o nó $A-V$ e seus ramos e a musculatura do septo interventricular, servindo de ponte antre estas estrutuiras, passando ao largo do feixe de His, conforme observaça em análise anatomo-patológica. Muitas décadas após sua descrição original, quando outros autores obserwaram vias acessónias atrioventriculares com condução lenta e propriedade decremental, eles fizeram a suposição de que o substrato para sua observação seria as fibras descritas por Mahaim.

Duas das estruturas originalmente descritas por Mahaim, as fibras nodoventriculares e as nodofasciculares, são raros e apenas objeto de relatos esporádicos. A maioria dos pacientes com vias acessórias com condução anterógrada decremental são hoje em dia classificadas como feixes atriofasciculares ou vias acessótias atrioventriculares longas com propriedade decremental. Estes pacientes geralmente apresentam uma taquicardia pré-excitada com QRS com configuraçăo tipo bloqueio de rano esquerdo. Nosso objetro no Capítulo 2 foi discorrer sobre a diagnóstico diferencial de umat pacientes com vias acessórias com condução anterógrada decremental são hoje em dia classificadas como feixes atriofasciculares ou vias acessórias atrioventriculares longas com propriedade decremental. Estes pacientes geralmente apresentam uma taquicardia pré-excitada com QRS com configuraça tipo bloqueio de ramo esquerdo. Nosso objetivo no Capítulo 2 foi discorrer sobre o diagnóstico diferencial de uma taquicardia com QRS alargado e padra tipo bloqueio do ramo esquerdo (BRE) enfatizando aspectos eletrocardiográficos potencialmente úteis no reconhecimento de arrimias tais como: uma taquicardia supraventricular com aberância tipo $\mathrm{BRE}$, uma taquicardia pré-excitada devido a uma via acessória com condução rápida localizada no anel tricuspide, uma taquicardia prexexcitada devido a uma vla acessória à direita com condugto lenta e uma taquicardia ventricular. Nớs também discutimos caracteristicas eletrocardiográficas sugestivas de mecanismos especificos de taquicardia ventricular como aquelas decorrentes de uma cicatriz miocádica pós isquemica, reentrada ramo-a-ramo e reentrada interfascicular, taquicardia ventricular 
direita idiopática e taquicardia ventricular associada à displasia ventricullar arritmogênica.

O eletrocardiograma de pacientes com feixes atriofasciculares é habiualmente descrito como normal. Um padrão de preexcitação mimima ja foi reconhecido por alguns autores, que, no entanto mão descreveran qualquer sinal eletrocardiográfico especifico de uma fibra de Mahaim. Nós analisamos um coorte de 40 pacientes com fibras de Mahaim, descrevendo no Capítulo 3 nossas observaçós à cerca das características eletrocardiográlicas durante ritmo sinusal e taquicardia pré-excitada. Este trabalho se detem em 33 pacientes, pois, foram excluidos 5 pacientes com vias acessórias manifestas (múltiplas vias) alèm de 2 pacientes com feixes atrioventriculares decrementais curtos. Nos observamos em $70 \%$ dos pacientes durante ritmo sinusal a presença de um achado eletrocardiográfico na derivaça III consistindo de um complexo QRS com configuração IS ou rSR', geralmente associado com ausência de onda $q$ septal nas derivações esquerdas. Näo há registro prévio deste achado na literatura especializada. Este padrão rS também foi observado em 4 dos 5 pacientes com sindrome de WPW associado à fibra de Mahaim, após a ablação da via acessónia com condução rápida. Houve uma correlação direta entre a presença deste padrão en III e desvio do eixo para a esquerda durante taquicardia preexcitada. Este fato aliado à observaçăo de que havia uma nítida moditicaçầ do complexo QRS na derivação III após a ablação da fibra de Mahaim (geralmente surgia um complexo tipo $\mathbf{q R}$ ou QR), validou o conceito de que a configuraça representava um sinal eletrocardiografico compativel com uma pré-excitação minima da ativação do ventriculo direito.

Em decorrência de nossa experiência apreendida com a análise desta extensa série de pacientes com fibras de Mahaim, nós descrevemos de forma sistemática no Capítulo 4, as diferentes taquiarritmias que podem ocorrer em um paciente portador de fibra de Mahaim. Descrevemos todas as variedades ben como sinais eletrocardiográficos que possam sugerir seu diagnóstico.

No Capítulo 5 testamos a sensibilidade, especificidade e o valor preditivo de critérios eletrocardiográficos previamente descritos para o diagnóstico de uma taquicardia associada à fibra de Mahaim em 40 pacientes. $O$ seu valor preditivo foi avaliado em un grupo de 35 pacientes com taquicardia supraventricular com morfologia de BRE causadas por taquicardia $\mathrm{A} . \mathrm{V}$ ortodromica taquicardia por reentrada nodal e taquicardia atrial. $O$ menor valor preditivo loi observado nos pacientes com taquicardia por mowimento circular utilizando uma via acessória oculta. Neste grupo observamos uma taxa de $26 \%$ de diagnósticos falso positivos (5 de 19 pacientes), en outras palavras, $26 \%$ teriam sido erroneamente diagnosicados como uma taquicardia utilizando uma via decremental (valor preditiwo negativo de $82,5 \%$ ). Os ariterios utilizados no trabaho de Bardy et al foram sensiveis apenas para os feixes atriofasciculares $(87,5 \%)$, não tendo sido úteis na deteç̧ăo de vias acessórias decrementais atrioventriculares $(0 \%)(p<0,0001)$.

No Capítulo 6 nốs analisamos o eletrocardiograma em ritmo sinusal de 7 pacientes com feixe faseiculoventricular e os comparamos com o ECG de 20 pacientes com via acessória anteroseptal e com 20 pacientes com via acessória medioseptal. No ECG de pacientes com um feixe fasciculoventricular a complexo QRS era mais estreito $(0.12+0.02)$ que o QRS de pacientes com vias acessórias septais $(0.14 \pm 0.01)$ 
$(p<0,0001)$. Nossos achados mostram uma superposição dos achados ao ECG entre os pacientes analisados, e num paciente com feixe fasciculoventricular com QRS $>0.12$ segundos nä̌ foi possivel discriminá-lo de pacientes com vias acessónias septais, o que sugere a necessidade de se realizar estudo eletrofisiológico para um diagnóstico correto.

No Capítulo 7 nós relatamos pela primeira vez a utilização de uma fibra de Mahaim (feixe atriofascicular) numa taquicardia não reentrante com uma relação $P / Q R S$ de 1:2. O substrato usual para uma taquicardia com relaça P:QRS de $1: 2$ é a presença de duas conexöes entre os átrios e os ventriculos. Neste caso especifico havia apenas uma conexão envolvida. A fisiopatologia estava relacionada com uma dissociação longitudinal da condução do impulso dentro da fibra de Mahaim. Condução dual por uma libra de Mahaim devido à dissociação longitudinal já foi objeto de relato para se justificar a presença de alternância do ciclo de uma taquicardia antidrômica, mas nunca anteriomente relacionada com uma conduçăo $1: 2$.

As vias fasciculoventriculares são amplamente discutidas no Capftulo 8 . A despeito dă năo participação ativa em circuitos reentrantes, nós enfatizamos a importância de um correto diagnóstico diferencial entre estas vias em uma via acessória anteroseptal parahissiana, e desta forma evitar dano ao nó $\mathrm{A}-\mathrm{V}$, no caso de uma tentativa equivocada de se realizar uma ablaç̃̃o neste tipo de conexão anômala. A possibilidade de erro diagnóstico é aumentada pelo fato de que existe uma alta incidencia de vias acessórias atrioventriculares associadas aos feixes fasciculoventriculares (nós publicamos um caso com 2 vias anômalas associadas). Descrevemos as características eletrocardiográficas e eletrofisiológicas e apresentamos uma revisäo da limitada literatura sobre o assunto.

Nós avaliamos a significado do automatismo induzido pela ablação por radiofiequência das fibras de Mahaim no Capitullo 9. Este fenômeno tem sido relatado mas não estudado de formá sistematizada. Nós descrevemos as características eletrocardiográticas do que denominamos de "Taquicardia automática de Mahaim" (MAT), sua relaçăo com o dano termo-induzido da porção proximal da fibra de Mahaim, sua semelhança com a taquicardia juncional automática que ocorre durante a ablaçio da via nodal lenta, sua importancia como um marcador de sucesso do procedimento ablativo, e sugerimos pela primeira vez, que em alguns pacientes pode ser necessário continuar a aplicação de radiofrequểncia até o término de toda a atividadie automática onginária da via decremental, para a obtençăo de resultado definitivo.

O Capítulo 10 é um relato de caso onde descrevemos nossos achados num paciente portador de uma fibra de Mahaim, submeticlo a ablaçăo por cateter com radiofrequência na inserçẫo distal da via. Nossos achados foram consistentes con uma fibra de Mahaim longa do tipo atrioventricular, se inserindo na musculatura da região antero-apical do ventriculo direito e não na porção distal do ramo direito do feixe de His, como seria o caso de um feixe atriofascicular.

Nosso objetivo no Capítulo 11 foi de descrever a ocorrência de automatismo espontâneo nas fibras de Mahaim, o que incluiu fenômenos diversos, desde automatismo termo-induzido durante a ablaçăo por radiofrequencia, ritmos automáticos lentos assintomáticos, ritmos automáticos espontâneos que trigam uma taquicardia antidrômica sob a influência de um tônus adrenérgico aumentado e ritmos 
automáticos espontâneos rápidos se apresentando como taquicardia repetitiva com carácter incessante. Nós discutimos estas arritmias autonáticas traqando uma analogia com ritmos automáticos nodais. Baseados nestes achados, nós concluimos que as fibras de Mahaim tem uma estrutura semelhante à do no $\mathrm{A}-\mathrm{V}$.

No Capítulo 12 nós estudamos as fibras curtas de Mahaim, denominaçáo aplicada a vias acessorias com conduçăo lenta e decremental com inserção ventricular justaanulares. As características clinicas de 8 pacientes com fibras curtas de Mahain bem como seu perfil eletrocardiográfico e eletrofisiologico foram comparados com um grupo de 33 pacientes com fextes atriofasciculares ou atrioventriculares longos. Além das impontantes diferenças entre os 2 grupos, observamos que os pacientes com fibras curtas não são um grupo homogeneo. Pode-se classifica-los em 2 grupos: um onde os pacientes apresentam caracteristicas de um nó A-V acessório e outro onde estas características estão ausentes.

\section{CONCLUSÕES GERAIS}

1. As variantes de pré-excitação "fibras de Mahaim" são estruturas raras com substratos anatômicos diferentes tendo como denominador" comum uma característica eletrofisiológica: condução unidirecional anterógrada lenta e decremental do estímulo elétrico, seja em decorrencia de propriedades intrinsecas da fibra, compativeis com um nó A-V acessório, fibras musculares ordinárias com condução lenta por fibrose secundária a ablação prévia ou por caracteristicas anatomicas especiais, ou ainda devido à conexão proximal desta fibra ao nó $\mathrm{A}-\mathrm{V}$ ou sistema His-Purkinje. Em trabalhos ja publicados, nós apresentamos evidencias adicionais sugerindo que algumas destas fibras de Mahaim näo se inserem no ramo direito do feixe de His, além das fibras curtas que se inserem no anulo valvar tricúspide.

2. Elas podem se manifestar como uma via acessoria isolada ou estar associada com vias acessórias com condução rápida. Ellas podem estar envolvidas em una grande variedade de arrimias distintas, algumas vezes com circuitos complexos. Descrevemos pela primeira vez ama variedade de laquicardia năo reentiante causada por uma conduçăo $1: 2$ do estímulo sinusal. Apenas a variedade fasciculowentricular das fibras de Mahaim näo participa ativamente em um circuito arritmogênico.

3. As fibras de Mahaim curtas habitualmente se manifestan com um $1 \mathrm{CG}$ indistinguivel de uma via acessória de condução rápida, exceto quando co-existe Lum intervalo PR normal (29\%). O diagnóstico eletrocardiográfico de uma fibra de Mahaim longa durante ritmo sinusal é possivel em até $70 \%$ dos pacientes. Seu peculiar padrão de pré-excitaçăo minima (rS) é melhor observado em na derivação III, e este padrão de QRS quando se associa à ausencia de onda $q$ na derivação I, parece ser bastante especifico para uma fibra de Mahaim. Pode-se suspeitar de uma taquicardia com conduçăo anterógrada por uma fibra de Mahaim

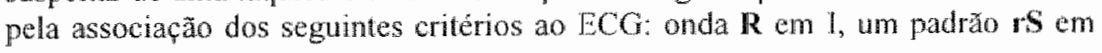


$V 1$, eixo do QRS no plano frontal entre 0 e -75 , transiçăo do QRS (R/S $<1$ ) além de V4 un QRS com largura menor que 0,15 segundos. $O$ valor preditivo negarivo destes critérios foi de $82,5 \%$ e a sensibilidade para o diagnóstico de feixes atriofasciculares foi de $87 \%$. Eles näo foram úteis para identificar uma taquicardia associada com fibras de Mahaim tipo atrioventriculares.

4. Automatismo espontâneo $e$ induzido durante ablação por radiofrequência originado em um feixe atriofasciculat ou em um feixe atrioventricular curto ou longo com condução decremental são características que sugerem que estas fibras sâo estruturas compativeis com um nó $\mathrm{A}-\mathrm{V}$ acessónio. 


\section{Samenvatting en Conclusies}

In hoofdstuk 1 wordt een overzicht gegeven van de belangrijkste publicaties over ongewone vormen (varianten) van ventriculaire pre-excitatie met de nadruk op diagnose en behandeling.

Volgens "Webster's New Dictionary" wordt met de naam variant aangegeven dat iets een beetje verschilt van de gebruikelijke of standaard vorm. De naam Mahaim bundel geldt voor een groep extra verbindingen die verschillen in hun proximale en distale insertie, maar gemeen hebben dat hun electrofysiologisch gedrag lijkt op dat van de A-V knoop. De naam Mahaim bundel of vezel vertelt ons dus meer over de elecrofysiologische eigenschappen dan de anatomische locatie en structuur.

Ivan Mahaim beschreef verbindingen tussen de $\mathrm{A}-\mathrm{V}$ kmoop en de bundeltakken of met het kamermyocard. Toen andere auteurs extra verbindingen beschreven met trage geleiding, zoals we die kennen van de $\mathrm{A}-\mathrm{V}$ knoop, gaven zijn hier aan ook de naam Mahaim bunde of vezel. Wij weten nu dat de nodoventriculaire en nodofasciculaire verbindingen die Mahaim beschreef zeldzaam zijn. Bij de meeste patienten thet een anterograad geleidende extra verbinding, is dit een verbinding tussen de rechter boezem en de rechter bundel (atriofasciculair) of et is sprake van een langzaam geleidende verbinding tussen de boezem en de rechterkamerspier (atrioventriculair). Belangrijk is dat wanneer deze patienten een tachycardie hebben met anterograde geleiding over de Mahain bundel het QRS complex tijdens de tachycardie een linker bundeltak blok vorm heeft. In hoofdstuk 2 wordt de differentiaal diagnostiek beschreven van een tachycardie met een linker bundeltak blok vorm. Aangegeven wordt hoe men kan herkennen of het hier een supraventriculaire tachycardia met linker bundeltak blok betreft, een tachycardie met snelle of langzame geleiding over een rechts gelegen extra verbinding, of een kamer tachycardie.

Ook wordt de aandach gevestigd op ECG bevindingen die bij wen linker bundel tak achtige VT wijzen op een ischemische of idiopathische origine, de aanwezigheid van een arthythmogene rechter kamer dysplasie of een tachyeardie die berust op re-entry in het bundeltak systeem.

In hoofdstuk 3 worden de ECG bevindingen beschreven, zowell bij sinus rime als tijdens de tachycardie, bij 33 patienten met een Mahaim bundel. Hicrbij wordt aandacht gevraagd voor een niet eerder beschreven bevinding dat tijdens sinus ritme het $E \mathrm{CG}$ in afleiding $I I I$ in $70 \%$ van de gevallen een $r S$ of rSR patoon laat zien, meestal gecombineerd met een afwezige $q$ in I, AVL en V6. Dit $\mathrm{TS}$ patroon in afleiding III werd ook gezien na ablatie wan een snel geleidende extra $\mathrm{A}-\mathrm{V}$ verbinding in 4 van de 5 patienten die naast een Maham bundel een WPW syndroom hadden. Er was een positieve relatie tussen een $\mathrm{rS}$ patroon in afleiding III Uijdens sinus ritme en linker as deviatic van het QRS complex tjidens een tachycardie met anterograde geleiding over de Mahaim bundel.

Deze bevinding en het feit dat na succesvolle ablatie van de Mahaim bundel een andere QRS configuratie optrad in afleiding III (meestal $q R$ of QR) onderstentit 
de gedachte dat het $\mathbf{S}$ patroon in afleiding III electrocardiografisch aangeeft dat er geringe pre-excitatie bestaat van het laag lateraal gelegen deel van de rechter ventrikel.

In hoofdstuk 4 worden de verschillende soorten tachycardie beschreven die kunnen woorkomen bij een patient met een Mahaim bundel.

Moofdstuk 5 beschrijft de toetsing van vroeger door Bardy en collega's beschreven ECG criteria voor een Mahaim tachycardie aan het eigen patienten matterial. Hierbij werden de Mahaim patienten vergeleken met 35 patienten met verschillende soorten supra ventriculaire tachycardie met een linker bundel tak blok. De eerder door Bardy gepubliceerde criteria waren het minst bruikbaar wannect een linker bundeltak blok aanwezig was bij patienten met een orthodrome cirkel tachycardie met ventriculo-atrieele geleiding over een extra A$V$ verbinding. Bij deze groep patienten werd met behulp van de Bardy criteria in $26 \%$ (5 van de 19 patienten) een wals positieve diagnose van een Mahaim vezell gesteld. De Bardy criteria hadden een sensitiviteit van $87,5 \%$ voor atriofasciculaire bundels, maar waren niet bruikbaar om de aanwezigheid van een langzaam geleidende $\mathrm{A}-\mathrm{V}$ extra verbinding te diagnostiseren.

In hoofdstuk 6 wordt het electrocardiogram tijdens sinus ritme beschreven van 7 patienten met een fasciculo ventriculaire extra verbinding. Deze ECG's werden vergeleken met die van 20 patienten met een antero septale en 20 patienten met een mid septale extra verbinding. Het QRS complex van patienten met een fasciculo ventriculaire extra verbinding was minder breed $(0,12 \pm 0,02 \mathrm{sec}$.) dan het QRS complex bij de septale AV extra verbindingen $(0,14 \pm 0,01 \mathrm{sec}$.) $(\mathrm{p}<$ $0,0001)$. Overigens bestaat er "overlap" in de ECG bevindingen tussen fasciculaoventriculaire extra verbindingen en antero septale en mid septale AV verbindingen. Dit laatste maakt dat wanneer er een verbreed QRS complex wordt gevonden, differentiering niet goed mogelijk is en dus een electrophysiologische studie noodzakelijk is om te komen tot een juiste diagnose.

Hoofdstuk 7 beschrijft een patient waarbij een tachycardie aanwezig was tijdens sinus ritme ondat iedere P-top werd gevolgd door 2 QRS complexen. Dit bleek te berusten op de aanwezigheid van longitudinale dissociatie in een Mahaim bundel. De verschillende geleidingstijden in de twee paden in de Mahaim bundel makte het mogelijk dat een $P$-top resulteerde in 2 QRS complexen. Dit is een nog niet eerdere beschreven vorm van tachycardie in een patient met een Mahaim bundel.

Hoofdstuk 8 gaat over fasciculo ventriculaire verbindingen. Ofschoon zij geen rol spelen als onderdeel van een tachycardie circuit is correcte identificatie van belang met name voor de

differentiatie met rechts gelegen anteroseptale para-hissale extra verbindingen. Een juiste diagnose voorkomt een ablatie poging met groot gevaar voor beschadiging van het $\mathrm{A}-\mathrm{V}$ geleidings systeem. Een foute diagnose kan gemaklkelik worden gemaakt omdat patienten met fasciculo ventriculaire vezels vaak ook accessoire $A-V$ verbindingen hebben en dus ook tachycardieen. Aandach wordt dan ook besteed aan de electrocardiografische en en electrofysiologische kenmerken van fasciculo ventriculaire vezels.

Zoals in hoofdstuk 9 beschreven kan tijdens radiofrequente cather ablatie van een Mahaim vezel vaak automatische impuls vorming in de Mahaim vezel worden geinduceerd. De ECG karakteristieken van de Mahaim automatische tachycardie (MAT) worden gepresenteend en de betekenis ervan besproken. Bij sommige patienten is het belangrijk on de radiofrequente ablatie van de Mahaim vezel zolang voor te zetten tot iedere automaticiteit is uitgedoofd. 
In hoofdstuk 10 wordt een patient besproken waarbij ablatie van de Maham bundel plaats vond aan het ventriculaire einde. Het ging hierbij om een lange atrio-ventriculaire Mahaim bundel die insereerde in het apicale deel van de rechter ventrikel en dus niet in de rechter bundel zoals bij de atrio-fasciculaire Mahaim vezel.

Hoofdstuk 11 geeft een overzicht van de verschillende vormen watonder automaticiteit in de Mahaim vezel zich kan manifesteren. Dit gedrag lijkt sterk op de automaticiteit zoals wij die kennen in de $A-V$ knoop en pleit dus voor een $A-V$ nodale opbouw van de Mahaim vezel.

In hoofdstuk 12 worden 8 patienten beschreven mot een korte A-V Mahaim verbinding. Klinische, electrocardiografische en electrofysiologische bevindingen bij deze patienten werden vergeleken met een groep van 33 patienten met een atrio fasciculaire verbinding of een lange $A-V$ extra verbinding met decrementale geleiding. De 33 patienten hadden een zeer homogeen electrofysiologisch profiel. Dit was niet het geval bij patienten met een korte $\mathrm{A}-\mathrm{V}$ Mahaim verbinding. Bij hen was er sprake wan 2 groepen. Bij de ene groep had de extra verbinding A-V nodale eigenschappen, bij de andere groep was dit niet het geval.

\section{CONCLUSIES}

1) De groep van Mahaim exira verbindingen bestaat uit verschillende anatomische substraten met als gemeenschappelijke electrophysiologische eigenschap: unidirectionele, langzame en decrementale antegrade geleiding. Dit kan verschillende oorzaken hebben, zoals A-V nodale eigenschappen van de extra verbinding, anisotrope geleiding in spicrvezells of een proximale verbinding met de $A-V$ knoop of het his - Purkinje systeem. Het is niet zo dat alle Mahaim extra verbindingen insereren in de rechter bundel van het intraventriculaire geleidingssysteem.

2) Mahaim extra verbindingen kunnen afzonderlijk voorkomen, maar worden wak gevonden samen met snel geleidende extra $A V$ verbindingen. $Z i j$ spelen een rol bij verschillende, soms zeer complexe, ritmestoomissein. Zij kunnen ook een tachycardie veroorzaken wanneer tijdens sinus ritme iedere P top gevolgd wordt door 2 QRS complexen door longitudinale dissociatie of duplicering van de Malaam verbinding. Fasciculo ventriculaire Mahaim verbindingen spelen geen rol in tachycardie circuits.

3) Het ECG van de patient met een korte AV Mahaim vezel is niet te onderscheiden van het ECG van een snel geleidende rechts gelegen exira AV verbinding behalve wanneer het P-delta interval notmaal is $(29 \%)$. In $70 \%$ van de patienten met een Mahaim extra verbinding is het nogelijk om de juiste diagnose te maken tijdens sinus titme. Dit berust op de aanwezigheid van een minimaal pre-excitatie ( $r S$ ) patroon in afleiding 11.1 , in het bijzonder wanneer dit gepaard gaat met de afwezigheid van een $q$ in afleiding $I$. De tachycardieen met anterograde geteiding over cen Mahaim. extra verbinding hebben meestal een $R$ in afleiding 1 , een $r S$ patroon in afleiding $V !$, een QRS as in het fromtale vlak tussen 0 en -75 graden, cen QRS R/S transitie in afleiding $V 4$ en een QRS breedte minder dan 0,15 seconden. De negatief voorspellende warde van deze criteria was $82,5 \%$ 
en de sensitiviteit $87 \%$ voor de aanwezigheid van atrio fasciculaire extra verbindingen. $2 j$ hadden geen waarde voor het diagnostiseren van tachycardien met anterograde geleiding over een atrio ventriculaire Mahaim vezel.

4) Automaticiteit, zowel spontaan als tijdens radiofrequente catheter ablatie, is en typisch verschijnsel bij Mahaim vezels. Het pleit voor de anwezigheid wan accessoir A-V nodaal weefsel. 


\section{Acknowledgements}

The bond between me and Professor Wellens teachings goes as far back as 1982 . At that time I was a resident in Cardiology, trying to keep the pace between my everyday duties, night calls and the study on books and joumals. Soon I became attracted to the study of cardiac arrhythmias. Looking for manuscripts in the leading journals of cardiology I came across many manuscripts of Hein Wellens and his group from Maastricht. I was profoundly impressed by his meticulous work, his unique didacticism specially the critical way he extracted information from the invasive eletrophysiologic studies to enhance the diagnostic yield of the electrocardiogram. As time went by it became clear for me that I would like to do a fellowship in Maastricht, and to benefit the most from it 1 did 1 year period of probation in Brazil. That was accomplished under the guidance of Professor Eduardo Sosa, head of one of the most important electrophysiologic laboratory in Brazil, at Instituto do Coração, São Paulo University. I am very much indebted to Dr Sosa , for his commitment to teaching, research and friendship.

The next step was to get in touch with Wellens, someone I only knew from the books and papers. Dr $A$. Belfer, a brazilian nuclear medicine specialist told me to contact Dr Frans Wackers, from Yale University, a close friend of Wellens. I wrote him a letter about my plans and his advise was crystal clear: write siraight to Wellens! Needless to say he immediately accepted me as a fellow, at my convenience. After a hard and long process I was granted a scholarship from CNPQ (Brazilian Council for Research) which enabled me to go to the Netherlands.

During my stay in Maastricht, I had the good fortune to attend the EP laboratory under the supervision of Dr Pedro Brugada and Dr Joep Smeets. Every ECG was a quiz session, and new observations on the tracings an inspiration for reports? Professon Wellens was always available for tête-amtete discussions on those difficult tracings that could resist so many brains eager to decipher them! I would like to recall the morning meetings sessions. I have greally benefit from the discussions and opinions of the staff members of the Afdeling Cardiologie as well as from informal discussions with the senior fellows at the "Provisorium" outbuilding in St Annadal Hospital, Maastricht, particularly from Mario Talajic, Roman Lezaun, Pelayo Torner, Hans Joachim Trappe, and Luz-Maria Rodriguez.

After conclusion of my fellowship I kept in touch with the department. In 1993, I came back to Mastricht for a 2 month period of training in catheter ablation techniques. I also attended the Congress "2000. Futwre of Amhythology: Lessons from the Past, Promises for Tomorrow", a rribute to the scientific legacy of Professor Wellens. 
In december of 2002 I went to Mastricht to attend some cryoablation procedures upon the invitation by Luz-Maria Rodriguez, a friend and ex-colleague from the fellowship program time. After reading some publications 1 did on variants of preexcitation she had motivated me to go on working on the subject. Professor Wellens opened the door again for me, and he would eventually become my promotor. Dr Carl Timmermans, a staff member of the Cardiology department kindly affered me the data he had collected from Mahaim patients over the years. I also am very grateful to Dr Ashish Nabar with his assistance with the dutch language on the analysis of the patient"s records as well as the thoughtful discussions about the cases from Maastricht. I soon realized that I was in need to increase the number of patients in the Mahaim database to enhance the power of my observations. I am indebted to Dr Eduardo Sosa, Instituto do Coraça ; São Paulo University, Brazil and Dr Femando E. Cruz. Filho and Marcio Fagundes from Instituto de Cardiologia Laranjeiras, Rio de Janeiro, Brazil, for allowing me to include their patients in my study. Dr Cruz was also a previous fellow in Maastricht. We spent many hours discussing issues related to mechanisms of cardiac arthythmias.

I would also like to express my gratitude toward Dr. Mário O. Vrandecic, president of the Biocor Institute and Dr. Bayard Gontijo Filho, clinical director, who foresee the great potential of electrophysiologic techniques in 1988 as well as to my own capabilities as a young electrophysiologist. They sponsored the implementation of the first clinical cardiac laboratory in the city of Belo Horizonte, Minas Gerais, Brazil.

I am also indebted to Dr. Antonio Luis de Oliveira Sobrinho and Dr. Mauricio Resende Barbosa, from the cath lab team, who showed endless patience in supporting me in those early days of the electrophysiology in Biocor Institute when I worked alone. Then I wish to thank Dr Luiz Gerken, my first fellow, now a skilful electrophysiologist, with whom 1 had the pleasiure to work with for more than 10 years.

This thesis could not have been done without the fabulous internet! There were countless e-mails between us. I was able to reach Hein sometimes more than once a day. Of course in the wake of an urgent demand by a reviewer the cellular phone was a last resource tool, and it worked wonderfully (Hein once told me - it seems you are next door...)

Dear Hein: Thank you for opening so many real and virtual doors and windows.

My last words (the first in my heart) of gratitude goes to my family, my wife Cláudia, and my sons Tomaz, lvan and Daniel for their patience, support and understanding for the time dedicated to the thesis instead of a trip, a tennis match, a walk in the pank.. 


\section{Curriculum Vitae}

\section{Eduardo Back Sternick}

May 6th, 1958

$1976-1981$

$1982-1984$

$1984-1986$

1985

$1986-1987$

1.987- 1988

1989

$1998-2002$

2003
Bom in Belo Horizonte, Brazil

Medical School, Universidade Federal de Minas Gerais (UFMG), Belo Horizonte, Brazil

Residency in Internal Medicine, Hospital das Clínicas, Faculdade de Medicina, UFMG, Belo Horizonte, Brazil

Residency in Cardiology, Instituto Dante Pazzaneze de Cardiologia, São Paulo, Brazil

Specialist in Intensive Medicine, São Paulo, Brazil

Research Fellow in Clinical Cardiac Electrophysiology, INCOR - Instituto do Coração, Universidade de São Paulo, São Paulo, Brazil

Research Fellow in Clinical Cardiac Electrophysiology, University' Hospital, Maastricht, The Netherlands

Director, Cardiac Electrophysiology Laboratory, Biocor Instituto, Nova Lima, Brazil

Director, Cardiac Electrophysiology Laboratory, Hospital Vera Cruz, Belo Horizonte, Brazil

Director, Cardiac Electrophysiology Laboratory, Santa Casa, Belo Horizonte, Brazil 


\section{Publications}

\section{Articles in Journals}

1) Rodriguez LM, Walefe A, Brugada P, Dehareng A, Lezam R, Sternick EB, Kulbertus HE: Exercise-Induced Sustained Symptomatic Ventricular Tachycardia. Incidence, Clinical, Angiographic, and Electrophysiologic Characteristics. Eur Heart J 1990; $11: 225-232$.

2) Sternick EB, Sobrinho ALA, Barbosa MR, Fantini F, Gontilo B, Vrandecic MO: Fibrilaçâo Ventricular en Portador de Displasia Ventricular e Prolapso Valvar Mitral. Arq Bras Cardiol 1990:55(5):311-314.

3) Sternick EB, Bahia FC, Gontijo B, Vrandecic MO: Miocardiopatia Induzida pôr Taquicardia Ventricular Incessante (taquicardiomiopatia). Cura após Controle da Arritmia. Arq Bras Cardiol 1992;58(3);209-14.

4) Sternick EB, Sobrinho ALA, Lisboa JCB, Barbosa MR, Fantini F, Gontijo B. Vrandecic MO: Ablaçåo Química Transcoronária de Taquicardia Ventricular em Portador de Cardiopatia Chagásica Crônica. Arq Bras Cardiol $1992 ; 58(4): 307-10$.

5) Fantini FA, Sternick EB, Gontijo B, Bracarense LF, Castro MF, Silva IAP, Barbosa JT, Vrandecic MO: Cirurgia para Sindrome de Wolff-ParkinsonWhite no $1^{\circ}$ Ano de Vida. Arq Bras Cardiol 1993;60(4):253-6

6) Sternick EB, Fantini FA, Borges LFQ, Gontijo B, Vrandecio MO: Conexão Atrio Fascicular com Condução Lenta e Dissociação Longitudinal. Comprovação Eletrofisiológica e Implicaçōes Clinicas. Arq Bras Cardiol $1993 ; 60(6): 4 \| 1-15$

7) Sternick EB, Gerken LM, Sobrinho ALO, Barbosa MR, Gontijo B. Vrandecic MO: Fibrilaçăo Ventricular em Paciente com Forma Permanente da Taquicardia Juncional Recipiocante. Arq Bras Cardiol 1996;66(1):25-27.

8) Rodrigues LM, Sternick EB, Smeets JLRM, Timmermans C, den Dulk K, Oreto $\mathrm{G}$, Wellens HJJ: Induction of Ventricular Fibrillation, But Not of Sustained Monomorphic Ventricular Tachycardia Predicts Sudden Death In Patients On Amiodarone Treatment Because of Ventricular Tachyarrhythmias Afier Myocardial Infarction. Heart 1996;75(1);23-8. 
9) Cruz FES, Fagundes ML, Boghossian SH, Ribeiro JCBM, Sternick E, Maia I: Ablação de Vias Anomalas com Condução Decremental. Arq̣ Bras Cardiol 1996,66 (Supl 1):39-44.

10) Stemick EB, Gerken LM, Barbosa MR, Sobrinho ALAO, Souza AC, Gontijo B, Vrandecic MO: Ablaça por Cateter com Radiofrequencia em Vias Acessórias Esquerdas: Abordagem Transaórtica Versus Transseptal. Reblampa 1997; 10:177-185.

11) Sternick EB, Gerken LM, God EG: Concealed Accessory Pathway with Long Conduction Times and Incremental Properties. A Case Report. I Cardiovaso Electrophysiol 2001;12 (1):103-106.

12) Sternick EB, Gerken LM, Vrandecic MO: Appraisal of "Mahaim" Automatic Tachycardia. J Cardiovase Electrophysiol 2002;13(3):244-249.

13) Stermick EB, Gerken LM, Vrandecic M, Wellens HJJ: Fasciculoventricular pathways. J Cardiovase Electrophysiol 2003;14:1057-1063.

14) Sternick EB: Mahaim Fibre Tachycardia: Recognition and Management. 1 Pacing and Electrophysiol J; 2003:3:47-59.

15) Sternick EB, Timmermans C, Sosa E, Cruz FE, Gerken LM, Scanavacca IM, Fagundes ML, Bueno SC, Vrandecic MO, Wellens HJJ: Automaticity in Mahaim fibers. J Cardiovasc Electrophysiol 2004;15:738-744.

16) Sternick EB, Gerken LM, Max R, Vrandecic MO: Radiofrequency Catheter Ablation of an Accessory Pathway in a Patient With WPW and Kartagener's Syndrome. Pacing Clin Electroplyysiol 2004; 27:401-3.

17) Sternick EB, Sosa E, Scanavacca M, Wellens HJy: Dual Conduction in a Mahaim Fiber. Cardiovase Electroplysiol 2004;15:1212-1215.

18) Sternick EB, Timmermans C, Sosa E, Cruz Filho FE, Rodriguez LM, Gerken LM, Fagundes M, Wellens HIJ: The electrocardiogram during sirus rhythm and during tachycardia in patients with anterograde conduction over Mahaim fibers. The role of the "rs" pattern in lead III. "Am Coll Cardiol $2004 ; 44: 126-135$.

19) Sternick EB, Cruz Filho FE, Timmermans C, Sosa $\mathbb{E}_{\text {, Rodriguez LM, }}$ Gerken LM, Fagundes M, Wellens H.J. The Electrocardiogram during Tachycardia in Patients with Anterograde Conduction over a Mahaim Fiber. Old Criteria Revisited. Heart Rhythm 2004;1(4):406-413. 
20) Sternick EB, Timmemans C, Rodriguez LM, Wellens HJJ: Mahaim fiber: an atriofascicular or a long atrioventricular pathways? (Accepted for publication in the Heart Rhythm joumal).

21) Sternick $\mathbb{E B}_{*}$ Fagundes $M$, Cruz Filho FE, Timmermans C, Sosa E, Rodriguez LM, Gerken LM, Scanavacea MI, Wellens HJJ: Short atrioventricular Mahaim fibers: observations on their clinical, electrocardiographic and electrophysiologic profile. (Accepted for publication in the Joumal of Cardiovascular Electrophysiology).

22) Sternick EB, Gerken LM, Cardoso AF. A consult for incessant palpitations. What is the rhythm? (Accepted for publication in the Joumal of Cardiovascular Electrophysiology).

23) Sternick EB, Rodriguez LM, Gerken LM, Wellens HJJ: The Electrocardiogram in patients with fasciculoventricular pathways: a comparative study with anteroseptal and midseptal accessory pathways. (Accepted for publication in the Heart Rhythm journal). 


\section{Chapters in Books}

1) Sosa E, Moffa P, Sternick E, Pileggi F. Alteraçoês De Ritmo e Da Conduçâo Do Estimulo. In: Serro-Azul LGCC, Pileggi FJC, Moffa PJ (eds). Propedeutica Cardiologica. Bases fisiopatológicas. Rio de Janeiro, Editora Guanabara Koogan SA, Rio de Janeiro, Brazil, 1988;387-391.

2) Sternick, EB. Taquicardias Wentriculares Incessantes. In: Cruz Filho F, Maia IG (eds). Eletrofisiologia e Clinica Das Arritmias Cardiacas. Editora Revinter, São Paulo, Brazil, 1997;233-239.

3) Sternick EB, Gerken LMS. Sindrome do QT longo. In: Porto CC (Ed). Doenças do Coraçăo. Prevenção e Tratamento. Editora Guanabara Koogan AS, Rio de Janeiro, Brazill, 1998;298-300.

4) Sternick EB, Gerken LM. Fibrilação atrial. Envelhecimento, álcool a hipertireoidismo. In : Ribeiro Moreira DA (ed): Fibrilaçăo Arrial, Lemos Editorial, São Paulo, Brazil 2003; 115-127. 